\title{
A Meldrum's Acid-Derived Thione Dienophile in a Convergent and Stereoselective Synthesis of a Tetracyclic Quassinoid Intermediate
}

\author{
Stéphane Perreault and Claude Spino*
}

Supporting Information

Experimental Procedures ................................................................................................................ 3

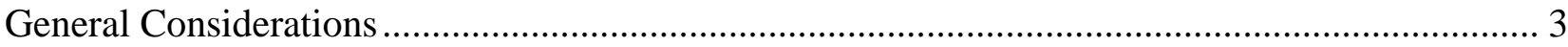

3-(tert-Butyldimethylsilyloxy)-1-propyne (9) ……………….................................................. 3

(5E)-1-(tert-Butyldimethylsilyloxy)-5-hepten-2-yn-4-one (10) .................................................... 3

( \pm )-(5E)-4-Acetoxy-1-(tert-butyldimethylsilyloxy)-4-(1,3-dithian-2-yl)-5-hepten-2-yne (11)........ 4

( \pm )-(5E)-4-(1,3-Dithian-2-yl)-2-methyl-2,3,5-heptatrien-1-ol (12) via alcohol 31.......................... 5

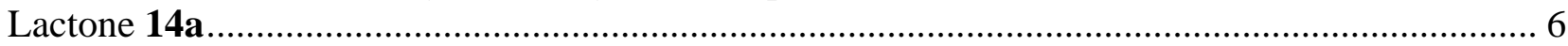

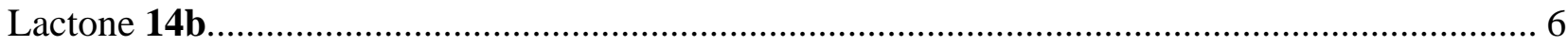

$\alpha, \beta$-Unsaturated aldehyde 15a

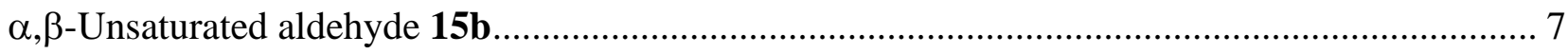

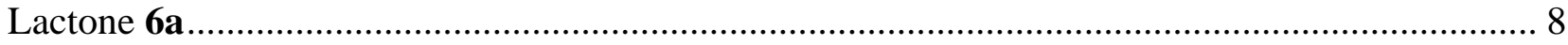

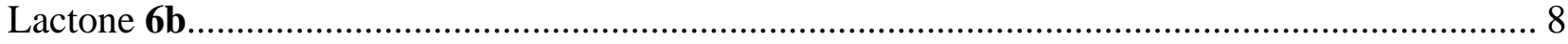

Triene $7 \mathbf{a}$

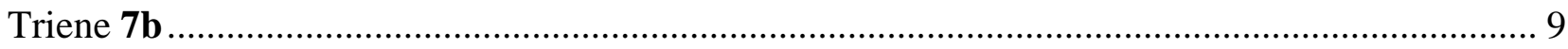

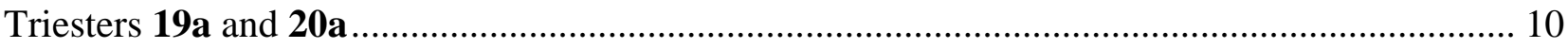

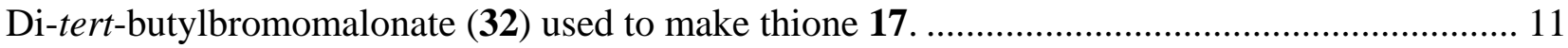

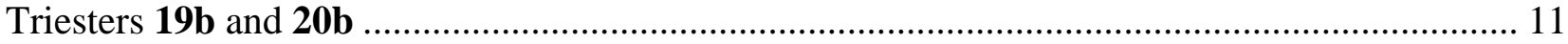

5-bromo-2,2-dimethyl-[1,3]dioxane-4,6-dione (33) used to make thione 18................................. 12

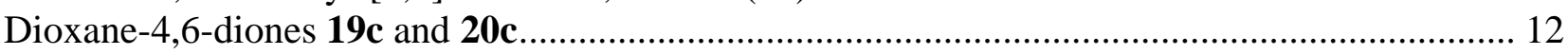

5-(phtalimide- $N$-sulfenyl)-2,2-dimethyl-[1,3]dioxane-4,6-dione (34) used to make thione 18. .... 13

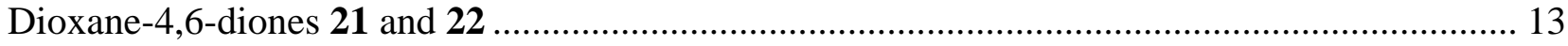

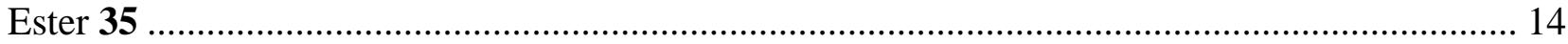

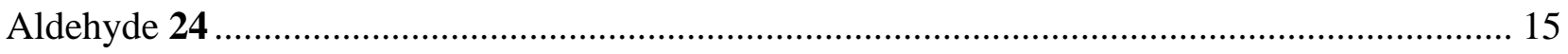

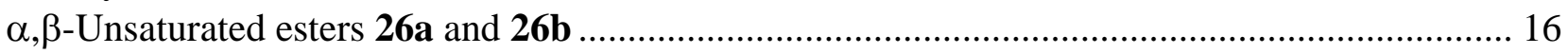

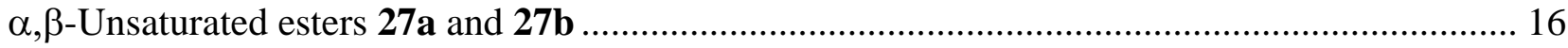

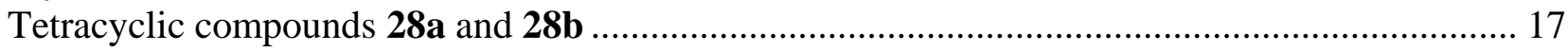

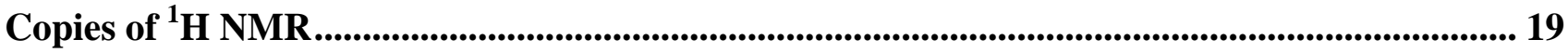

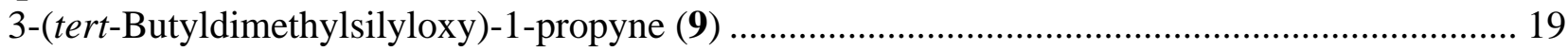

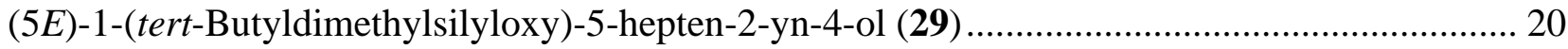

(5E)-1-(tert-Butyldimethylsilyloxy)-5-hepten-2-yn-4-one (10) .................................................. 21

( \pm )-(5E)-1-(tert-butyldimethylsilyloxy)-4-(1,3-dithian-2-yl)-5-hepten-2-yn-4-ol (30)................... 22

( \pm )-(5E)-4-Acetoxy-1-(tert-butyldimethylsilyloxy)-4-(1,3-dithian-2-yl)-5-hepten-2-yne (11)...... 23

( \pm )-(5E)-4-(1,3-Dithian-2-yl)-2-methyl-2,3,5-heptatrien-1-ol (12) .................................................. 24

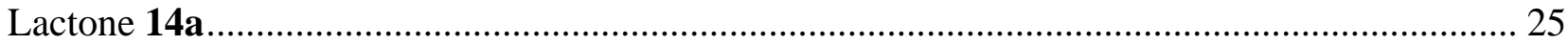

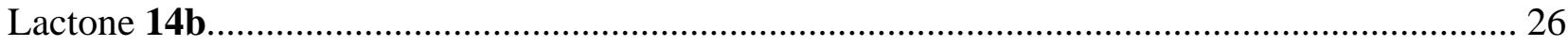

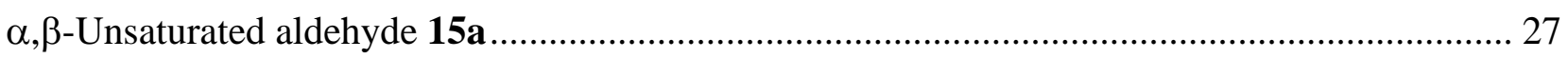




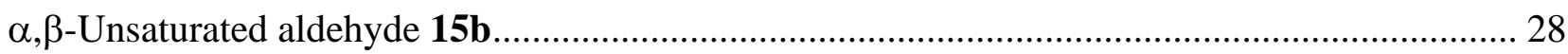

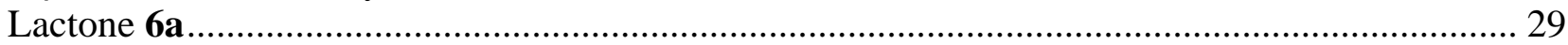

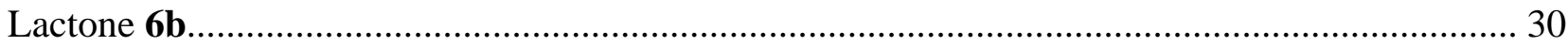

Triene $7 \mathbf{a}$

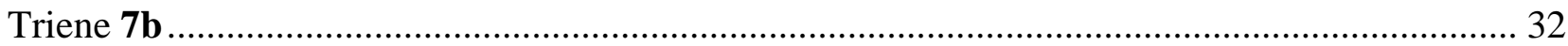

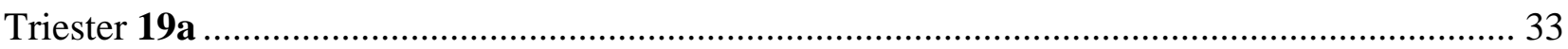

Triester 20a

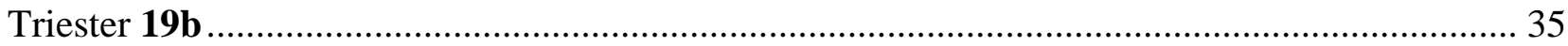

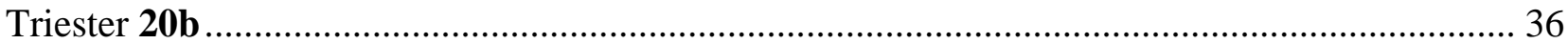

5-bromo-2,2-dimethyl-[1,3]dioxane-4,6-dione (33) used to make thione 18.................................. 37

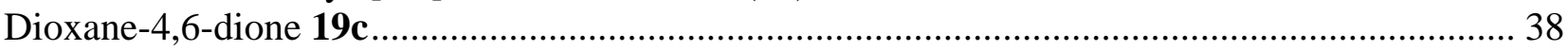

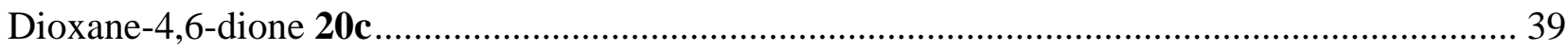

5-(phtalimide- $N$-sulfenyl)-2,2-dimethyl-[1,3]dioxane-4,6-dione (34) used to make thione 18. .... 40

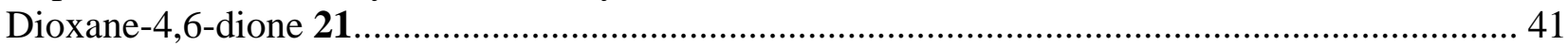

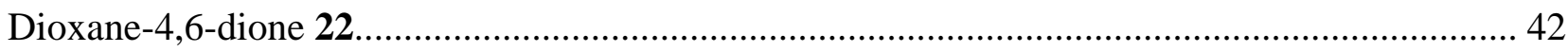

Ester 35 (minor diastrereomer) .................................................................................................. 43

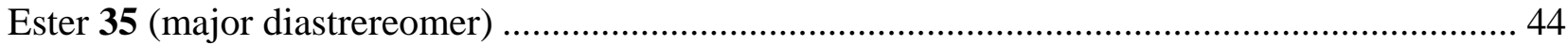

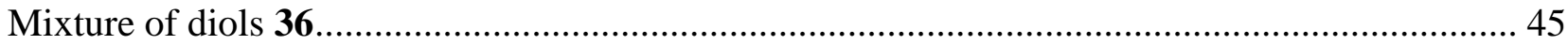

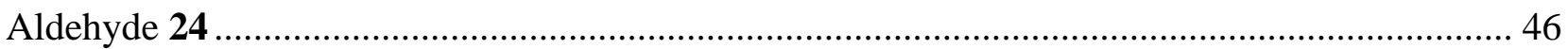

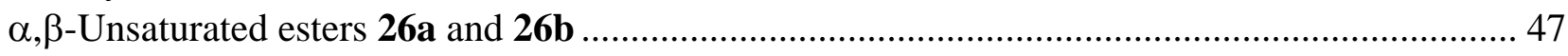

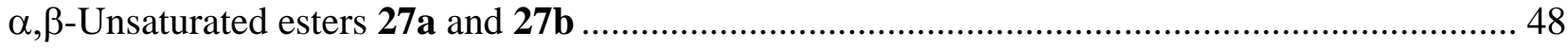

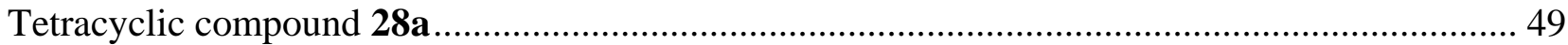

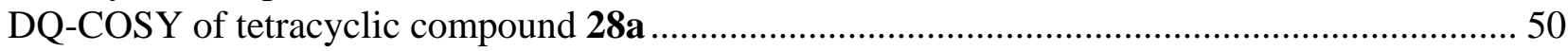

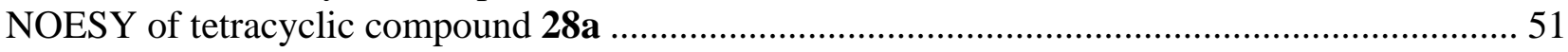

Tetracyclic compound 28b.................................................................................................. 52

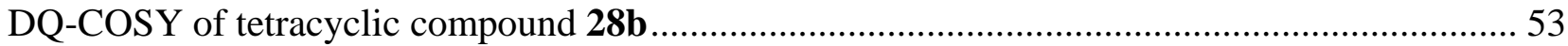

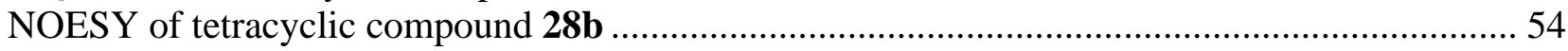

Summary of X-Ray Crystallographic Data ......................................................................................... 55

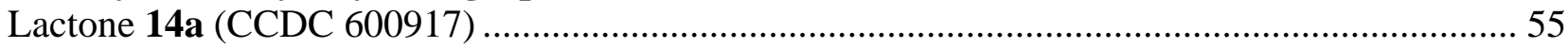

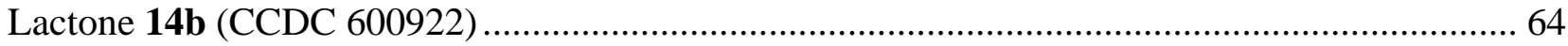

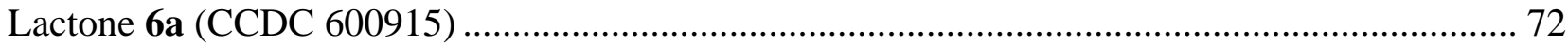

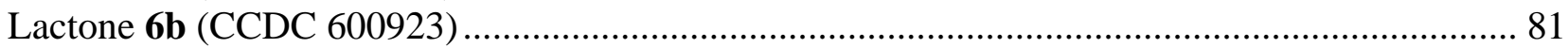

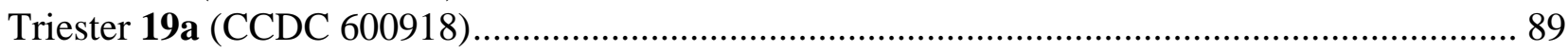

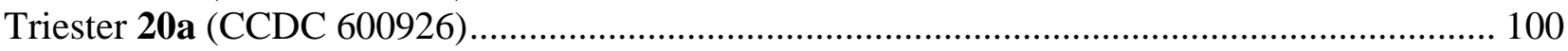

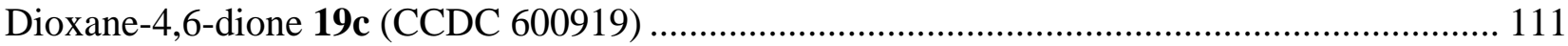

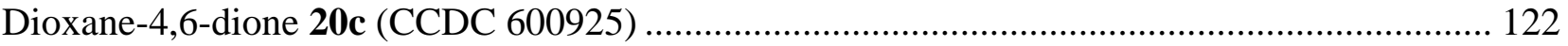

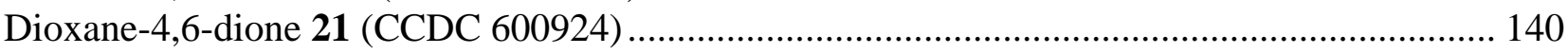




\section{Experimental Procedures}

\section{General Considerations}

All reactions were performed under an inert atmosphere of argon in glassware that had been flame dried. Solvents were distilled from potassium/benzophenone ketyl $\left(\mathrm{Et}_{2} \mathrm{O}\right.$, tetrahydrofuran, benzene, toluene), from calcium hydride $\left(\mathrm{CH}_{2} \mathrm{Cl}_{2}\right.$, triethylamine, pyridine, diisopropylamine) prior to use. Reagents were purchased from Aldrich and used without purification. Grignard and alkyl lithium reagents were titrated by the method of Love and Jones. ${ }^{[1]}$ Flash chromatography was performed using Merck silica gel (230-400 Mesh ASTM) with solvents distilled prior to use. NMR spectra were recorded on a Bruker AC-300 spectrometer $\left({ }^{1} \mathrm{H} \mathrm{NMR}\right.$ at $300 \mathrm{MHz},{ }^{13} \mathrm{C} \mathrm{NMR}$ at $75.5 \mathrm{MHz}$ ). The following abbreviations were used: s, singlet, d, doublet, t, triplet, q, quartet. Chemical shifts are reported in ppm with the solvent resonance as the internal standard $\left(\mathrm{CDCl}_{3}: 7.26 \mathrm{ppm}\left({ }^{1} \mathrm{H} \mathrm{NMR}\right), 77.0\right.$ ppm $\left.\left({ }^{13} \mathrm{C} N M R\right)\right)$. Infrared spectra were recorded on a Perkin Elmer 1600 FTIR spectrometer with a thin layer of the product on a $\mathrm{NaCl}$ disk. GCMS analyses were performed on a HP 5890 serie II instrument (25 m length, $25 \mu \mathrm{OD}$, capillary Stabiliwax-DB5 column) coupled with a mass spectrometer (HP 5971). High-resolution spectrometry was performed on a ZAB-1F micromass spectrometer.

\section{3-(tert-Butyldimethylsilyloxy)-1-propyne (9)}

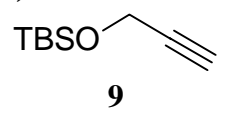

Propargyl alcohol (10.0 g, $178 \mathrm{mmol})$ was dissolved in dichloromethane (500 mL). Imidazole (30.4 g, $446 \mathrm{mmol}$ ) and tert-butyldimethylsilyl chloride (32.3 g, $214 \mathrm{mmol})$ were added at rt. The reaction mixture was stirred at rt for $30 \mathrm{~min}$ and quenched with water. The phases were separated and the aqueous phase was extracted twice with dichloromethane. The organic layers were combined, washed once with water, washed once with brine, dried over anhydrous magnesium sulfate and concentrated under reduced pressure to give a colourless liquid. The crude product 9 (30.1 g, 99\%) was used without purification for the next reaction. ${ }^{1} \mathbf{H}$ NMR $\left(\mathrm{CDCl}_{3}, 300 \mathrm{MHz}\right): \delta 4.30(\mathrm{~d}, 2 \mathrm{H}, J=2.5 \mathrm{~Hz}), 2.39(\mathrm{t}, 1 \mathrm{H}$, $J=2.5 \mathrm{~Hz}$ ), 0.91 (s, 9H), 0.12 (s, 6H). IR (neat, $\left.\mathrm{cm}^{-1}\right): 3435,1651$. LRMS $(\mathrm{m} / \mathrm{z}$, relative intensity):

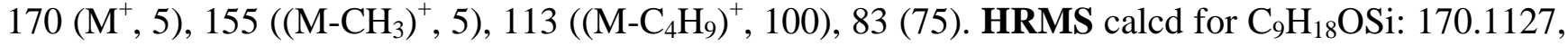
found: 170.1131 .

\section{(5E)-1-(tert-Butyldimethylsilyloxy)-5-hepten-2-yn-4-one (10)}

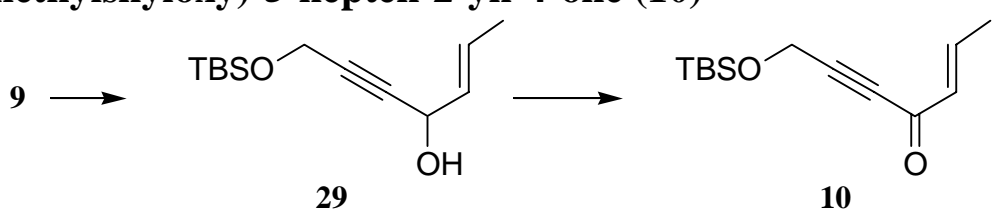

Protected propargyl alcohol 9 (24.1 g, $141 \mathrm{mmol}$ ) was dissolved in THF (550 mL) and the solution was cooled to $-78{ }^{\circ} \mathrm{C}$. A $2.36 \mathrm{M}$ solution of $n$-BuLi in hexanes $(65.8 \mathrm{~mL}, 135 \mathrm{mmol})$ was slowly added and the reaction mixture was stirred $10 \mathrm{~min}$ at $-78{ }^{\circ} \mathrm{C}$ and $15 \mathrm{~min}$ at $-10{ }^{\circ} \mathrm{C}$. The reaction mixture was cooled to $-78{ }^{\circ} \mathrm{C}$ before the addition of trans-crotonaldehyde $(11.1 \mathrm{~mL}, 134 \mathrm{mmol})$. The reaction mixture was allowed to warm to rt and stirred overnight. The reaction mixture was quenched at $0{ }^{\circ} \mathrm{C}$ with saturated aqueous $\mathrm{NH}_{4} \mathrm{Cl}$. The phases were separated and the aqueous phase was extracted three times with ethyl acetate. The organic layers were combined, washed once with water, washed once with brine, dried over anhydrous magnesium sulfate and concentrated under reduced pressure to give a yellowish liquid. The crude product 29 was purified by flash chromatography on a silica gel column eluting with $20 \%$ ethyl acetate in hexanes to give allylic alcohol 29 (33.5 g, 98\% from propargyl alcohol) as a colourless liquid. ${ }^{1} \mathbf{H}$ NMR $\left(\mathrm{CDCl}_{3}, 300 \mathrm{MHz}\right): \delta 5.80$ (dq, $1 \mathrm{H}, J=14.9$ and $\left.6.6 \mathrm{~Hz}\right), 5.53$ 
(dd, $1 \mathrm{H}, J=14.9$ and $6.6 \mathrm{~Hz}$ ), 4.79-4.74 (m, 1H), 4.28 (s, 2H), 3.03 (broad s, 1H), 1.64 (d, 3H, $J=6.6$ $\mathrm{Hz}$ ), 0.83 (s, 9H), 0.05 (s, 6H). ${ }^{13} \mathrm{C} \mathrm{NMR}\left(\mathrm{CDCl}_{3}, 75.5 \mathrm{MHz}\right): \delta 130.2$ (d), 128.2 (d), 84.4 (s), 84.1 (s), 62.5 (d), 51.6 (t), 25.7 (q), 18.2 (s), 17.3 (q), -5.2 (q). IR (neat, $\mathrm{cm}^{-1}$ ): 3620-3050 (broad), 3020, 2928,

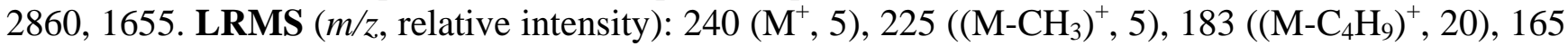
(15), 4 (100). HRMS calcd for $\mathrm{C}_{9} \mathrm{H}_{15} \mathrm{O}_{2} \mathrm{Si}\left(\mathrm{M}-\mathrm{C}_{4} \mathrm{H}_{9}\right)$ : 183.0841 , found: 183.0847.

Allylic alcohol 29 (15.0 g, $62.4 \mathrm{mmol})$ was dissolved in dichloromethane (300 mL) and $85 \%$ activated $\mathrm{MnO}_{2}$ (25.5 g, $250 \mathrm{mmol}$ ) was added at rt. The reaction mixture was stirred at rt for 3 days, filtered on celite and concentrated under reduced pressure to give a yellow liquid. The crude product $\mathbf{1 0}$ was purified by flash chromatography on a silica gel column eluting with 3\% to 5\% ethyl acetate in hexanes to give 10 (14.6 g, 98\%) as a colourless liquid. ${ }^{\mathbf{1}} \mathbf{H} \mathbf{~ N M R}\left(\mathrm{CDCl}_{3}, 300 \mathrm{MHz}\right): \delta 7.29$ (dq, $1 \mathrm{H}, J=15.7$ and $7.2 \mathrm{~Hz}$ ), 6.16 (dd, 1H, $J=15.7$ and $1.4 \mathrm{~Hz}$ ), 4.48 (s, 2H), 1.96 (dd, 3H, $J=7.2$ and $1.4 \mathrm{~Hz}$ ), 0.90 (s, 9H), 0.12 (s, 6H). ${ }^{13} \mathrm{C} \mathrm{NMR}\left(\mathrm{CDCl}_{3}, 75.5 \mathrm{MHz}\right)$ : $\delta 177.7$ (s), 149.9 (d), 133.5 (d), 90.6 (s), 81.9 (s), 51.4 (t), 25.6 (q), 18.3 (q), 18.1 (s), -5.4 (q). IR (neat, $\mathrm{cm}^{-1}$ ): 3022, 2936, 2857, 2213, 1630. LRMS $\left(\mathrm{m} / \mathrm{z}\right.$, relative intensity): $223\left(\left(\mathrm{M}-\mathrm{CH}_{3}\right)^{+}, 5\right), 181\left(\left(\mathrm{M}-\mathrm{C}_{4} \mathrm{H}_{9}\right)^{+}, 100\right), 151$ (50). HRMS calcd for $\mathrm{C}_{9} \mathrm{H}_{13} \mathrm{O}_{2} \mathrm{Si}\left(\mathrm{M}-\mathrm{C}_{4} \mathrm{H}_{9}\right)$ : 181.0685, found: 181.0681 .

\section{(士)-(5E)-4-Acetoxy-1-(tert-butyldimethylsilyloxy)-4-(1,3-dithian-2-yl)-5-hepten-2-yne (11)}

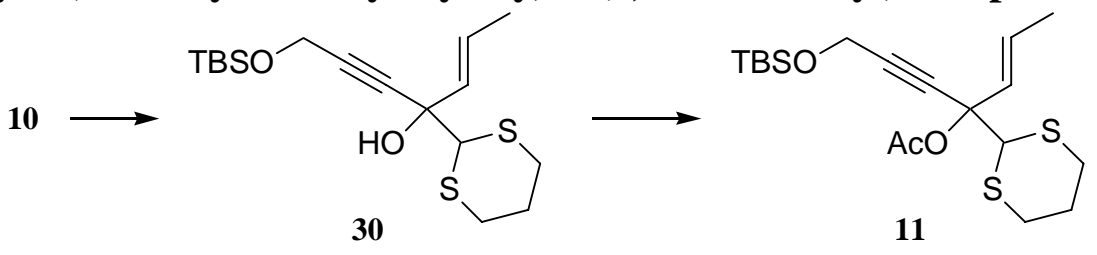

1,3-Dithiane (3.03 g, $25.2 \mathrm{mmol}$ ) was dissolved in THF (75 mL) and the solution was cooled to $-78{ }^{\circ} \mathrm{C}$. A $2.33 \mathrm{M}$ solution of $n$-BuLi in hexanes $(11.9 \mathrm{~mL}, 27.7 \mathrm{mmol})$ was added at $-78{ }^{\circ} \mathrm{C}$ and the reaction mixture was stirred at $-78{ }^{\circ} \mathrm{C}$ for $30 \mathrm{~min}$ and $30 \mathrm{~min}$ at $0{ }^{\circ} \mathrm{C}$. The reaction mixture was cooled to $-78{ }^{\circ} \mathrm{C}$ and a solution of unsaturated ketone 10 (6.01 g, $25.2 \mathrm{mmol})$ in THF (50 mL) was added. The reaction mixture was stirred at $-78{ }^{\circ} \mathrm{C}$ for $2.5 \mathrm{~h}$ and quenched with saturated aqueous $\mathrm{NH}_{4} \mathrm{Cl}$. The phases were separated and the aqueous phase was extracted twice with diethyl ether. The organic layers were combined, washed once with water, washed once with brine, dried over anhydrous magnesium sulfate and concentrated under reduced pressure to give a yellow oil. The crude product $\mathbf{3 0}$ was purified by flash chromatography on a silica gel column eluting with $3 \%$ to $8 \%$ diethyl ether in hexanes to give allylic alcohol 30 (5.73 g, 63\%) as a colourless oil. ${ }^{1} \mathbf{H}$ NMR $\left(\mathrm{CDCl}_{3}, 300 \mathrm{MHz}\right): \delta 6.16(\mathrm{dq}, 1 \mathrm{H}, J=$ 15.4 and $6.6 \mathrm{~Hz}$ ), 5.63 (dd, $1 \mathrm{H}, J=15.4$ and $1.7 \mathrm{~Hz}), 4.39(\mathrm{~s}, 2 \mathrm{H}), 3.83(\mathrm{~s}, 1 \mathrm{H}), 3.31-3.20(\mathrm{~m}, 3 \mathrm{H})$, 2.71-2.61 (m, 2H), 2.06-1.98 (m, 2H), 1.77 (dd, 3H, $J=6.6$ and $1.7 \mathrm{~Hz}), 0.89(\mathrm{~s}, 9 \mathrm{H}), 0.11(\mathrm{~s}, 6 \mathrm{H}) .{ }^{13} \mathrm{C}$ NMR $\left(\mathrm{CDCl}_{3}, 75.5 \mathrm{MHz}\right): \delta 130.8$ (d), 129.1 (d), 85.6 (s), 85.1 (s), $73.4(\mathrm{~s}), 54.2(\mathrm{~d}), 51.8(\mathrm{t}), 27.6$ (t), 25.7 (q), 25.0 (t), 17.4 (q), -5.2 (q). IR (neat, $\mathrm{cm}^{-1}$ ): 3571-3055 (broad), 2962, 2935, 2864, 2344. LRMS ( $\mathrm{m} / \mathrm{z}$, relative intensity): 358 ( $\left.\mathrm{M}^{+}, 5\right), 301$ (20), 239 (30), 226 (60), 119 (100). HRMS calcd for $\mathrm{C}_{17} \mathrm{H}_{30} \mathrm{O}_{2} \mathrm{~S}_{2} \mathrm{Si}: 358.1456$, found: 358.1453.

Allylic alcohol 30 (22.60 g, $63.2 \mathrm{mmol})$ was dissolved in dichloromethane (270 mL). Triethylamine (30.8 mL, $220.9 \mathrm{mmol}$ ), 4-(dimethylamino)pyridine (387 mg, $3.2 \mathrm{mmol}$ ) and acetic anhydride (17.8 $\mathrm{mL}, 189.5 \mathrm{mmol}$ ) were successively added at rt. The reaction mixture was stirred at rt for 3 days and quenched with saturated aqueous $\mathrm{NH}_{4} \mathrm{Cl}$. The phases were separated and the aqueous phase was extracted twice with dichloromethane. The organic layers were combined, washed once with water, washed once with brine, dried over anhydrous magnesium sulfate and concentrated under reduced pressure to give a yellow oil. The crude product $\mathbf{1 1}$ was found to be unstable on silica gel and was used without purification for the next reaction. Only a small amount of $\mathbf{1 1}$ was purified by flash 
chromatography on a silica gel column eluting with $5 \%$ ethyl acetate in hexanes for analytical purposes. ${ }^{1} \mathbf{H}$ NMR $\left(\mathrm{CDCl}_{3}, 300 \mathrm{MHz}\right): \delta 6.24$ (dq, $1 \mathrm{H}, J=15.2$ and $\left.6.6 \mathrm{~Hz}\right), 5.75$ (dd, $1 \mathrm{H}, J=15.2$ and $1.7 \mathrm{~Hz}$ ), 4.86 (s, 1H), 4.41 (s, 2H), 2.97-2.74 (m, 4H), 2.10-2.00 (m, 1H), 2.05 (s, 3H), 1.94-1.83 (m, 1H), 1.78 (dd, 3H, $J=6.6$ and $1.7 \mathrm{~Hz}), 0.89$ (s, 9H), 0.12 (s, 6H). ${ }^{13} \mathbf{C ~ N M R}\left(\mathrm{CDCl}_{3}, 75.5 \mathrm{MHz}\right): \delta$ $168.8(\mathrm{~s}), 131.9(\mathrm{~d}), 127.3(\mathrm{~d}), 87.8(\mathrm{~s}), 80.4(\mathrm{~s}), 79.6(\mathrm{~s}), 54.8(\mathrm{~d}), 51.8(\mathrm{t}), 30.0(\mathrm{t}), 25.7(\mathrm{q}), 25.6(\mathrm{t})$, 21.7 (q), 18.2 (s), 17.5 (q), -5.2 (q). IR (neat, $\mathrm{cm}^{-1}$ ): 2983, 2933, 2855, 1751. LRMS ( $\mathrm{m} / \mathrm{z}$, relative

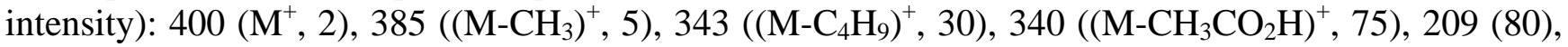
119 (95), 74 (100). HRMS calcd for $\mathrm{C}_{19} \mathrm{H}_{32} \mathrm{O}_{3} \mathrm{~S}_{2} \mathrm{Si}$ : 400.1562 , found: 400.1569.

( \pm )-(5E)-4-(1,3-Dithian-2-yl)-2-methyl-2,3,5-heptatrien-1-ol (12) via alcohol 31.

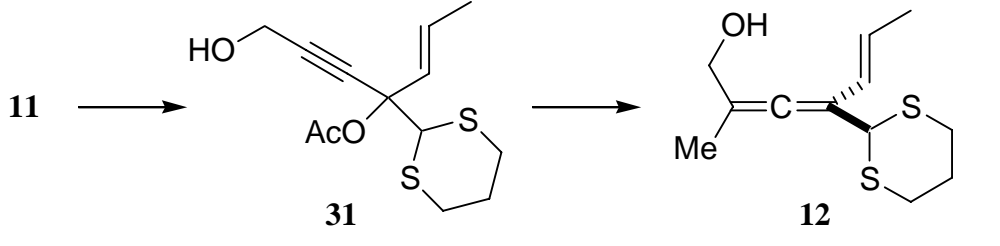

Acetate 11 (63.2 mmol) was dissolved in THF (315 mL) and cooled to $0{ }^{\circ} \mathrm{C}$. A 1.0 M solution tetrabutylammonium fluoride in THF $(95 \mathrm{~mL}, 95.0 \mathrm{mmol})$ was added at $0{ }^{\circ} \mathrm{C}$. The reaction mixture was stirred at $0{ }^{\circ} \mathrm{C}$ for $1 \mathrm{~h}$ and quenched with saturated aqueous $\mathrm{NH}_{4} \mathrm{Cl}$. The phases were separated and the aqueous phase was extracted twice with diethyl ether. The organic layers were combined, washed once with water, washed once with brine, dried over anhydrous magnesium sulfate and concentrated under reduced pressure to give a yellow oil. Alcohol $\mathbf{3 1}$ was found to be unstable on silica gel and was used without purification for the next reaction.

Anhydrous LiBr (16.5 g, $189 \mathrm{mmol})$ and CuI (36.1 g, $189 \mathrm{mmol})$ were dissolved in THF (200 mL) and the mechanically stirred solution was cooled to $-10^{\circ} \mathrm{C}$. A $2.64 \mathrm{M}$ solution of $\mathrm{MeMgBr}$ in diethyl ether (71.8 mL, $189 \mathrm{mmol}$ ) was slowly added. The reaction mixture was stirred $1 \mathrm{~h}$ at $-10^{\circ} \mathrm{C}$. A solution of $31(31.6 \mathrm{mmol})$ in THF $(115 \mathrm{~mL})$ was slowly added at $-10^{\circ} \mathrm{C}$. The reaction mixture was stirred $1 \mathrm{~h}$ at $10{ }^{\circ} \mathrm{C}$ and quenched with a 9:1 solution of saturated aqueous $\mathrm{NH}_{4} \mathrm{Cl}$ and $\mathrm{NH}_{4} \mathrm{OH}$. The phases were separated and the aqueous phase was extracted three times with diethyl ether. The organic layers were combined, washed once with water, washed once with brine, dried over anhydrous magnesium sulfate and concentrated under reduced pressure to give a yellow oil. The crude product $\mathbf{1 2}$ can be purified on a silica gel column eluting with 30\% ethyl acetate in hexanes to give $\mathbf{1 2}$ (58\% for 3 steps) as a colourless oil. For larger batches, 12 was used without purification for the next reaction. ${ }^{\mathbf{1}} \mathbf{H}$ NMR $\left(\mathrm{CDCl}_{3}, 300 \mathrm{MHz}\right): \delta$ 5.92-5.78 (m, 2H), $4.67(\mathrm{~s}, 1 \mathrm{H}), 4.08(\mathrm{~d}, 2 \mathrm{H}, J=6.1 \mathrm{~Hz}), 3.02-2.82(\mathrm{~m}, 4 \mathrm{H})$, 2.15-2.06 (m, 1H), 1.93-1.83 (m, 2H), $1.80(\mathrm{~s}, 3 \mathrm{H}), 1.78(\mathrm{~d}, 3 \mathrm{H}, J=5.0 \mathrm{~Hz}) .{ }^{13} \mathbf{C} \mathbf{~ N M R}\left(\mathrm{CDCl}_{3}, 75.5\right.$ MHz): $\delta 200.8(s), 126.6(d), 125.3(d), 108.4(s), 106.7(s), 63.8(t), 47.2(d), 31.6(t), 31.5(t), 25.5(t)$, 18.3 (q), 15.7 (q). IR (neat, $\mathrm{cm}^{-1}$ ): 3687-3105 (broad), 2899, 2348, 2323, 1633. LRMS (m/z,relative intensity): $242\left(\mathrm{M}^{+}, 5\right), 211$ (70), 137 (30), 119 (100). HRMS calcd for $\mathrm{C}_{12} \mathrm{H}_{18} \mathrm{OS}_{2}$ : 242.0799, found: 242.0803. 


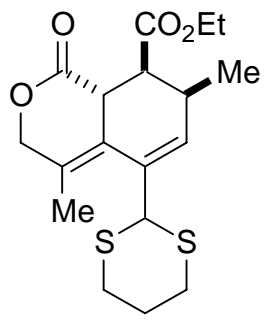

14a

Vinylallene 12 (31.6 mmol) was dissolved in benzene $(150 \mathrm{~mL})$ and the solution was cooled to $5{ }^{\circ} \mathrm{C}$. Fumaric acid monoethyl ester (5.00 g, $34.7 \mathrm{mmol})$, 4-(dimethylamino)pyridine (385 mg, $3.2 \mathrm{mmol})$ and $N, N^{\prime}$-dicyclohexylcarbodiimide $(7.20 \mathrm{~g}, 34.7 \mathrm{mmol})$ were successively added at $5{ }^{\circ} \mathrm{C}$. The reaction mixture was stirred at $5{ }^{\circ} \mathrm{C}$ for $1.5 \mathrm{~h}$ and at $80{ }^{\circ} \mathrm{C}$ for $8 \mathrm{~h}$. The reaction mixture was cooled to rt and filtered. The cake was washed with $\mathrm{CH}_{2} \mathrm{Cl}_{2}$ and the filtrate was concentrated under reduced pressure to give a brown solid. The solid was triturated under vigorous stirring in diethyl ether for $3 \mathrm{~h}$. The brownish solid 14a was filtered, dissolved in a 1:1 mixture of dichloromethane and ethyl acetate and passed through a silica gel pad eluting with a 1:1 mixture of dichloromethane and ethyl acetate to give 14a (6.41 g, 55\% for 4 steps) as a white solid. ${ }^{\mathbf{1}} \mathbf{H} \mathbf{~ N M R}\left(\mathrm{CDCl}_{3}, 300 \mathrm{MHz}\right): \delta 6.58(\mathrm{~d}, 1 \mathrm{H}, J=7.2 \mathrm{~Hz})$, 4.90 (d, 1H, $J=15.4 \mathrm{~Hz}), 4.63$ (s, 1H), 4.52 (d, $1 \mathrm{H}, J=15.4 \mathrm{~Hz}), 4.20$ (q, 2H, $J=7.2 \mathrm{~Hz}), 3.54$ (dm, $1 \mathrm{H}, J=11.0 \mathrm{~Hz}$ ), 2.99 (dd, $1 \mathrm{H}, J=11.0$ and $5.0 \mathrm{~Hz}$ ), 2.93-2.74 (m, 5H), $2.16(\mathrm{~s}, 3 \mathrm{H}), 2.14-2.10(\mathrm{~m}$, 1H), 1.91-1.76 (m, 1H), 1.28 (t, 3H, $J=7.2 \mathrm{~Hz}), 0.93$ (d, 3H, $J=7.2 \mathrm{~Hz}) .{ }^{13} \mathbf{C}$ NMR $\left(\mathrm{CDCl}_{3}, 75.5\right.$ MHz): $\delta 172.6$ (s), 172.4 (s), 139.2 (d), 133.9 (s), 127.8 (s), 124.8 (s), 71.0 (t), 60.8 (t), 48.5 (d), 42.7 (d), 40.8 (d), 32.9 (t), 32.7 (t), 31.0 (d), 25.0 (t), 17.8 (q), 15.3 (q), 14.2 (q). IR (neat, cm $\left.{ }^{-1}\right):$ 2975, 2931, 2904, 1731. LRMS (m/z,relative intensity): 368 ( $\left.\mathbf{M}^{+}, 100\right), 323$ (30), 247 (20), 219 (25), 187 (30), 145 (35). HRMS calcd for $\mathrm{C}_{18} \mathrm{H}_{24} \mathrm{O}_{4} \mathrm{~S}_{2}$ : 368.1116, found: 368.1106. m.p. ( $\left.{ }^{\circ} \mathrm{C}\right): 209-210$.

\section{Lactone 14b}

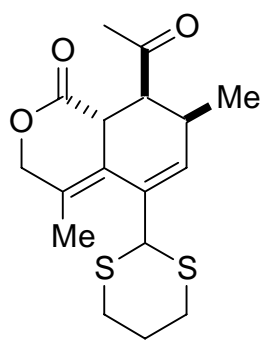

14b

Vinylallene $12(61.6 \mathrm{mmol})$ was dissolved in benzene $(300 \mathrm{~mL})$ and the solution was cooled to $5{ }^{\circ} \mathrm{C}$. 4 Oxo-2-pentenoic acid ${ }^{[2]}$ (7.72 g, $\left.67.6 \mathrm{mmol}\right)$, 4-(dimethylamino)pyridine $(752 \mathrm{mg}, 6.1 \mathrm{mmol})$ and $\mathrm{N}$, $\mathrm{N}^{\prime}$-dicyclohexylcarbodiimide $(14.0 \mathrm{~g}, 67.6 \mathrm{mmol})$ were successively added at $5{ }^{\circ} \mathrm{C}$. The reaction mixture was stirred at $5{ }^{\circ} \mathrm{C}$ for $1.5 \mathrm{~h}$ and at $80{ }^{\circ} \mathrm{C}$ for $2 \mathrm{~h}$. The reaction mixture was cooled to rt and filtered. The cake was washed with $\mathrm{CH}_{2} \mathrm{Cl}_{2}$ and the filtrate was concentrated under reduced pressure to give a brown solid. The solid was triturated under vigorous stirring in diethyl ether for $3 \mathrm{~h}$. The brownish solid 14b was filtered, dissolved in a 1:1 mixture of dichloromethane and ethyl acetate and passed through a silica gel pad eluting with a 1:1 mixture of dichloromethane and ethyl acetate to give 14b (10.7 g, 51\% for 4 steps) as a white solid. ${ }^{\mathbf{1}} \mathbf{H} \mathbf{~ N M R}\left(\mathrm{CDCl}_{3}, 300 \mathrm{MHz}\right): \delta 6.63(\mathrm{~d}, 1 \mathrm{H}, J=7.7 \mathrm{~Hz})$, 4.90 (d, 1H, $J=14.9 \mathrm{~Hz}$ ), 4.66 (s, 1H), 4.52 (d, 1H, $J=14.9 \mathrm{~Hz}$ ), 3.64 (dm, 1H, $J=11.5 \mathrm{~Hz}$ ), 3.12 (dd, $1 \mathrm{H}, J=11.5$ and $4.4 \mathrm{~Hz}), 3.00-2.79(\mathrm{~m}, 5 \mathrm{H}), 2.30(\mathrm{~s}, 3 \mathrm{H}), 2.17(\mathrm{~s}, 3 \mathrm{H}), 2.14-2.08(\mathrm{~m}, 1 \mathrm{H}), 1.93-1.81$ (m, 1H), 0.81 (d, 3H, $J=7.2 \mathrm{~Hz}) .{ }^{13} \mathrm{C}$ NMR $\left(\mathrm{CDCl}_{3}, 75.5 \mathrm{MHz}\right): \delta 207.8$ (s), 172.7 (s), 138.8 (d), 
134.0 (s), 128.0 (s), 124.7 (s), 71.0 (t), 48.8 (d), 48.4 (d), 40.2 (d), 32.9 (t), 32.7 (t), 30.1 (d), 29.5 (q), 25.0 (t), 17.7 (q), 14.8 (q). IR (neat, $\mathrm{cm}^{-1}$ ): 2972, 2886, 1733, 1708. LRMS ( $\mathrm{m} / \mathrm{z}$,relative intensity): 338 ( $\left.\mathrm{M}^{+}, 100\right), 221$ (30). HRMS calcd for $\mathrm{C}_{17} \mathrm{H}_{22} \mathrm{O}_{3} \mathrm{~S}_{2}: 338.1010$, found: 338.1016. m.p. $\left({ }^{\circ} \mathrm{C}\right): 238-240$.

\section{$\alpha, \beta$-Unsaturated aldehyde 15a}

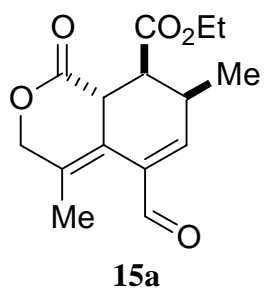

Lactone 14a (1.00 g, $2.7 \mathrm{mmol})$ was dissolved in dichloromethane $(18 \mathrm{~mL})$ and water $(0.5 \mathrm{~mL})$. DessMartin periodinane $(2.30 \mathrm{~g}, 5.4 \mathrm{mmol})$ was added at rt. The reaction mixture was stirred at $40{ }^{\circ} \mathrm{C}$ for 2 days. The reaction mixture was cooled to rt and quenched with a solution of $10 \% \mathrm{Na}_{2} \mathrm{~S}_{2} \mathrm{O}_{3}$ in saturated aqueous $\mathrm{NaHCO}_{3}$. The phases were separated and the aqueous phase was extracted three times with dichloromethane. The organic layers were combined, washed once with water, washed once with brine, dried over anhydrous magnesium sulfate and concentrated under reduced pressure to give yellow oil. The crude product 15a was purified by flash chromatography on a silica gel column eluting with $50 \%$ ethyl acetate in hexanes to give 15a (642 mg, 85\%) as a colourless oil. ${ }^{\mathbf{1}} \mathbf{H} \mathbf{~ N M R}\left(\mathrm{CDCl}_{3}, 300 \mathrm{MHz}\right): \delta$ $9.56(\mathrm{~s}, 1 \mathrm{H}), 7.02(\mathrm{~d}, 1 \mathrm{H}, J=6.6 \mathrm{~Hz}), 4.95$ (d, $1 \mathrm{H}, J=15.4 \mathrm{~Hz}), 4.61$ (d, $1 \mathrm{H}, J=15.4 \mathrm{~Hz}), 4.20$ (q, $2 \mathrm{H}, J=7.2 \mathrm{~Hz}$ ), 3.60-3.56 (m, 1H), 3.07 (dd, $1 \mathrm{H}, J=9.6$ and $4.4 \mathrm{~Hz}$ ), 3.01-2.90 (m, 1H), 1.85 (d, 3H, $J=2.2 \mathrm{~Hz}$ ), 1.27 (t, 3H, $J=7.2 \mathrm{~Hz}), 1.12$ (d, 3H, $J=7.0 \mathrm{~Hz}) .{ }^{13} \mathbf{C}$ NMR $\left(\mathrm{CDCl}_{3}, 75.5 \mathrm{MHz}\right): \delta 190.0$ (d), 171.9 (s), 171.7 (s), 153.3 (d), 136.9 (s), 132.7 (s), 120.2 (s), 70.8 (t), 61.1 (t), 43.2 (d), 40.8 (d), 32.0 (d), 19.4 (q), 14.9 (q), 14.2 (q). IR (neat, $\mathrm{cm}^{-1}$ ): 2983, 2930, 2860, 1739. LRMS ( $\mathrm{m} / \mathrm{z}$, relative intensity): 278 ( $\left.\mathrm{M}^{+}, 35\right), 233$ (70), 204 (100), 159 (55). HRMS calcd for $\mathrm{C}_{15} \mathrm{H}_{18} \mathrm{O}_{5}$ : 278.1154, found: 278.1160 .

\section{$\alpha, \beta$-Unsaturated aldehyde 15b}

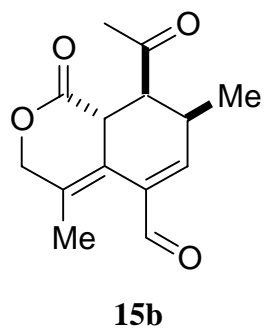

Lactone $14 \mathbf{b}(21.1 \mathrm{~g}, 62.3 \mathrm{mmol})$ was dissolved in dichloromethane $(300 \mathrm{~mL})$ and water $(10 \mathrm{~mL})$. Dess-Martin periodinane (52.8 g, $124.6 \mathrm{mmol}$ ) was added at rt. The reaction mixture was stirred at 40 ${ }^{\circ} \mathrm{C}$ for 2 days. The reaction mixture was cooled to rt and quenched with a solution of $10 \% \mathrm{Na}_{2} \mathrm{~S}_{2} \mathrm{O}_{3}$ in saturated aqueous $\mathrm{NaHCO}_{3}$. The phases were separated and the aqueous phase was extracted three times with dichloromethane. The organic layers were combined, washed once with water, washed once with brine, dried over anhydrous magnesium sulfate and concentrated under reduced pressure to give yellow oil. The crude product $\mathbf{1 5 b}$ was purified by flash chromatography on a silica gel column eluting with $50 \%$ ethyl acetate in hexanes to give $\mathbf{1 5 b}(12.5 \mathrm{~g}, 81 \%)$ as a white solid. ${ }^{\mathbf{1}} \mathbf{H} \mathbf{~ N M R}\left(\mathrm{CDCl}_{3}, 300\right.$ MHz): $\delta 9.58(\mathrm{~s}, 1 \mathrm{H}), 7.06(\mathrm{~d}, 1 \mathrm{H}, J=7.2 \mathrm{~Hz}), 4.93(\mathrm{dd}, 1 \mathrm{H}, J=15.4$ and $2.5 \mathrm{~Hz}), 4.61(\mathrm{~d}, 1 \mathrm{H}, J=$ $15.4 \mathrm{~Hz}$ ), $3.68(\mathrm{dm}, 1 \mathrm{H}, J=11.0 \mathrm{~Hz}), 3.12(\mathrm{dd}, 1 \mathrm{H}, J=11.0$ and $4.4 \mathrm{~Hz}), 3.06-2.96(\mathrm{~m}, 1 \mathrm{H}), 2.32(\mathrm{~s}$, 3H), 1.85 (d, 3H, $J=2.5 \mathrm{~Hz}), 0.97(\mathrm{~d}, 3 \mathrm{H}, J=7.2 \mathrm{~Hz}) .{ }^{13} \mathbf{C}$ NMR $\left(\mathrm{CDCl}_{3}, 75.5 \mathrm{MHz}\right): \delta 206.9(\mathrm{~s})$, 190.2 (d), 172.1 (s), 152.0 (d), 136.7 (s), 133.0 (s), 120.1 (s), 70.8 (t), 48.7 (d), 39.9 (d), 30.9 (d), 29.7 
(q), 19.3 (q), 14.0 (q). IR (neat, $\mathrm{cm}^{-1}$ ): 2973, 2916, 2847, 1745, 1711, 1699. LRMS (m/z, relative intensity): $248\left(\mathrm{M}^{+}, 5\right), 231$ (20), 213 (10), 205 (15). HRMS calcd for $\mathrm{C}_{14} \mathrm{H}_{16} \mathrm{O}_{4}$ : 248.1048, found: 248.1044. m.p. ( $\left.{ }^{\circ} \mathrm{C}\right): 110-111$.

\section{Lactone 6a}

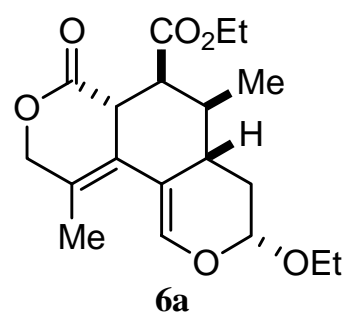

$\alpha, \beta$-Unsaturated aldehyde 15a (656 mg, $2.36 \mathrm{mmol}$ ) was dissolved in ethyl vinyl ether (12.0 $\mathrm{mL}$ ). Argon was bubbled in this solution for $15 \mathrm{~min}$ and $\mathrm{Yb}(\mathrm{fod})_{3}(375 \mathrm{mg}, 0.35 \mathrm{mmol}$ ) was added. The flask was purged 3 times with argon. The reaction mixture was stirred at rt for 4 days and concentrated to give a yellowish oil. The crude product 6 a was purified by flash chromatography on a silica gel column eluting with $15 \%$ to $20 \%$ ethyl acetate in hexanes to give $\mathbf{6 a}(612 \mathrm{mg}, 74 \%)$ as a white solid. ${ }^{\mathbf{1}} \mathbf{H}$ NMR $\left(\mathrm{CDCl}_{3}, 300 \mathrm{MHz}\right): \delta 6.30$ (d, $1 \mathrm{H}, J=2.2 \mathrm{~Hz}$ ), 5.07 (dd, $1 \mathrm{H}, J=8.5$ and $2.2 \mathrm{~Hz}$ ), 4.88 (dd, $1 \mathrm{H}, J=$ 14.9 and $2.2 \mathrm{~Hz}$ ), 4.55 (d, 1H, $J=14.9 \mathrm{~Hz}$ ), 4.24-4.11 (m, 2H), 3.90 (dq, 1H, $J=9.6$ and 7.2 Hz), 3.57 (dq, $1 \mathrm{H}, J=9.6$ and $7.2 \mathrm{~Hz}$ ), 3.36 (dm, $1 \mathrm{H}, J=11.5 \mathrm{~Hz}$ ), 2.83 (dd, $1 \mathrm{H}, J=11.5$ and $4.4 \mathrm{~Hz}$ ), 2.26-2.05 (m, 3H), 1.91 (d, 3H, $J=2.2 \mathrm{~Hz}), 1.83-1.72(\mathrm{~m}, 1 \mathrm{H}), 1.27$ (t, 3H, $J=7.2 \mathrm{~Hz}), 1.24$ (t, 3H, $J=7.2 \mathrm{~Hz}$ ), 0.93 (d, 3H, $J=7.2 \mathrm{~Hz}) .{ }^{13} \mathrm{C}$ NMR $\left(\mathrm{CDCl}_{3}, 75.5 \mathrm{MHz}\right): \delta 173.1$ (s), 172.4 (s), 142.1 (d), 127.2 (s), 124.5 (s), 108.6 (s), 99.2 (d), 70.7 (t), 64.2 (t), 60.6 (t), 42.6 (d), 40.5 (d), 36.4 (d), 34.4 (d), 34.2 (t), 16.9 (q), 15.1 (q), 14.1 (q). IR (neat, $\mathrm{cm}^{-1}$ ): 2976, 2939, 2918, 1738. LRMS ( $\mathrm{m} / \mathrm{z}$, relative intensity): 350 (M+, 15), 304 (95), 260 (40), 204 (70), 187 (75), 159 (100). HRMS calcd for $\mathrm{C}_{19} \mathrm{H}_{26} \mathrm{O}_{6}$ : 350.1729, found: $350.1736 . \mathbf{m . p} .\left({ }^{\circ} \mathrm{C}\right): 137-139$.

\section{Lactone 6b}

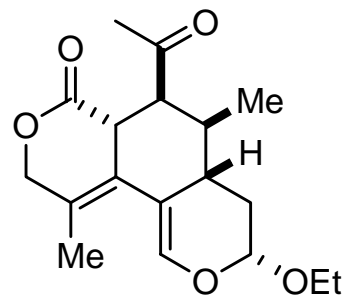

6b

$\alpha, \beta$-Unsaturated aldehyde 15b (115 mg, $0.46 \mathrm{mmol}$ ) was dissolved in benzene (2.5 mL) and ethylvinyl ether $(2.5 \mathrm{~mL})$. Argon was bubbled in this solution for $10 \mathrm{~min}$ and $\mathrm{Yb}(\mathrm{fod})_{3}(98 \mathrm{mg}, 0.09 \mathrm{mmol})$ was added. The flask was purged 3 times with argon. The reaction mixture was stirred at rt for 3 days and concentrated to give a yellowish oil. The crude product $\mathbf{6 b}$ was purified by flash chromatography on a silica gel column eluting with $15 \%$ to $20 \%$ ethyl acetate in hexanes to give $\mathbf{6 b}(144 \mathrm{mg}, 97 \%$ ) as a white solid. ${ }^{1} \mathbf{H}$ NMR $\left(\mathrm{CDCl}_{3}, 300 \mathrm{MHz}\right): \delta 6.29$ (d, $\left.1 \mathrm{H}, J=2.2 \mathrm{~Hz}\right), 5.09$ (dd, $1 \mathrm{H}, J=8.0$ and 2.2 $\mathrm{Hz}$ ), 4.88 (dd, 1H, $J=14.8$ and $2.2 \mathrm{~Hz}$ ), 4.52 (d, 1H, $J=14.8 \mathrm{~Hz}$ ), 3.91 (dq, 1H, $J=9.6$ and $7.2 \mathrm{~Hz}$ ), 3.58 (dq, $1 \mathrm{H}, J=9.6$ and $7.2 \mathrm{~Hz}$ ), 3.43 (dm, $1 \mathrm{H}, J=12.0 \mathrm{~Hz}$ ), 3.00 (dd, $1 \mathrm{H}, J=12.0$ and $4.4 \mathrm{~Hz}$ ), 2.27 (s, 3H), 2.22-2.09 (m, 3H), 1.91 (d, 3H, $J=2.2 \mathrm{~Hz}$ ), 1.85-1.75 (m, 1H), 1.25 (t, 3H, $J=7.2 \mathrm{~Hz}$ ), 0.83 (d, 3H, $J=7.2 \mathrm{~Hz}) .{ }^{13} \mathrm{C}$ NMR $\left(\mathrm{CDCl}_{3}, 75.5 \mathrm{MHz}\right): \delta 208.5$ (s), 172.9 (s), 142.0 (d), 127.9 (s), 124.7 (s), 108.6 (s), 98.9 (d), 70.6 (t), 64.3 (t), 48.3 (d), 40.6 (d), 36.6 (d), 34.4 (t), 33.3 (d), 29.7 (q), 16.8 (q), 
16.7 (q), 15.1 (q). IR (neat, $\mathrm{cm}^{-1}$ ): 2977, 2920, 2879, 1744, 1714, 1622. LRMS (m/z, relative intensity): 320 ( $\mathrm{M}^{+}$, 25), 274 (100), 231 (90), 205 (40), 187 (80), 159 (100). HRMS calcd for $\mathrm{C}_{18} \mathrm{H}_{24} \mathrm{O}_{5}$ : 320.1624, found: 320.1630 . m.p. $\left({ }^{\circ} \mathrm{C}\right): 189-190$.

\section{Triene 7a}

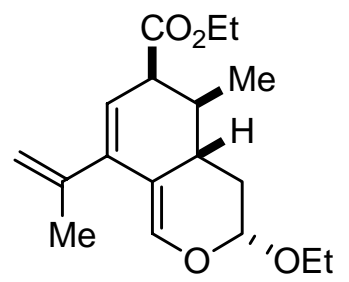

7a

$\alpha, \beta$-Unsaturated aldehyde 15a (12.5 g, $44.9 \mathrm{mmol})$ was dissolved in benzene (225 mL). Argon was bubbled in this solution for $15 \mathrm{~min}$ and ethyl vinyl ether $(49 \mathrm{~mL}, 674 \mathrm{mmol})$ was added. Argon was bubbled in this solution for $5 \mathrm{~min}$ and $\mathrm{Yb}(\mathrm{fod})_{3}(6.2 \mathrm{~g}, 5.9 \mathrm{mmol})$ was added. The flask was purged 3 times with argon. The reaction mixture was stirred at $80{ }^{\circ} \mathrm{C}$ for $24 \mathrm{~h}$ and concentrated to give a yellow oil. The crude product 7a was purified by flash chromatography on a silica gel column eluting with $10 \%$ dichloromethane in hexanes followed by $5 \%$ ethyl acetate in hexanes to give $7 \mathbf{a}(9.87 \mathrm{~g}, 72 \%)$ as a colourless oil. ${ }^{1} \mathbf{H}$ NMR $\left(\mathrm{CDCl}_{3}, 300 \mathrm{MHz}\right): \delta 6.41$ (d, $\left.1 \mathrm{H}, J=1.6 \mathrm{~Hz}\right), 5.29(\mathrm{~d}, 1 \mathrm{H}, J=6.0 \mathrm{~Hz}), 4.98$ (dd, $1 \mathrm{H}, J=9.9$ and $2.2 \mathrm{~Hz}), 4.94$ (s, 1H), 4.82 (s, 1H), 4.18-4.03 (m, 2H), 3.94 (dq, $1 \mathrm{H}, J=9.4$ and $7.2 \mathrm{~Hz}$ ), 3.59 (dq, $1 \mathrm{H}, J=9.4$ and $7.2 \mathrm{~Hz}$ ), 3.14 (t, $1 \mathrm{H}, J=6.0 \mathrm{~Hz}$ ), 2.84 (tdd, $1 \mathrm{H}, J=11.5$, 5.5 and 1.6 Hz), 2.17 (ddd, $1 \mathrm{H}, J=12.6,5.5$ and $2.2 \mathrm{~Hz}$ ), 1.86 (s, 3H), 1.67-1.52 (m, 1H), 1.38 (td, $1 \mathrm{H}, J=12.6$ and $9.9 \mathrm{~Hz}), 1.25(\mathrm{t}, 3 \mathrm{H}, J=7.2 \mathrm{~Hz}), 1.24(\mathrm{t}, 3 \mathrm{H}, J=7.2 \mathrm{~Hz}), 1.01(\mathrm{~d}, 3 \mathrm{H}, J=6.6 \mathrm{~Hz}) .{ }^{13} \mathbf{C} \mathbf{~ N M R}$ $\left(\mathrm{CDCl}_{3}, 75.5 \mathrm{MHz}\right): \delta 172.9$ (s), 144.3 (s), 141.5 (s), 140.8 (d), 116.7 (d), 114.5 (t), 112.2 (s), 100.3 (d), $64.5(\mathrm{t}), 60.2$ (t), 47.3 (d), 35.6 (d), 33.8 (t), 31.8 (d), 23.4 (q), 15.7 (q), 15.1 (q), 14.2 (q). IR (neat, $\mathrm{cm}^{-1}$ ): 3157,3077, 3035, 3016, 2980, 2945, 1717, 1625. LRMS (m/z, relative intensity): $306\left(\mathrm{M}^{+}, 5\right)$, 293 (20), 247 (50), 173 (30), 159 (30), 86 (100). HRMS calcd for $\mathrm{C}_{18} \mathrm{H}_{26} \mathrm{O}_{4}$ : 306.1831, found: 306.1825.

\section{Triene $7 \mathbf{b}$}

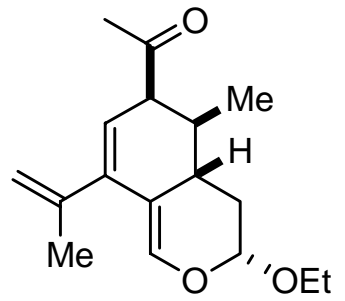

$7 \mathbf{b}$

$\alpha, \beta$-Unsaturated aldehyde 15b (12.5 g, $50.3 \mathrm{mmol})$ was dissolved in benzene (225 $\mathrm{mL})$. Argon was bubbled in this solution for 30 min and ethyl vinyl ether $(49 \mathrm{~mL}, 674 \mathrm{mmol})$ was added. Argon was bubbled in this solution for $5 \mathrm{~min}$ and $\mathrm{Yb}(\mathrm{fod})_{3}(10.6 \mathrm{~g}, 10.0 \mathrm{mmol})$ was added. The flask was purged 3 times with argon. The reaction mixture was stirred at $80^{\circ} \mathrm{C}$ for $24 \mathrm{~h}$ and concentrated to give a yellow oil. The crude product $\mathbf{7 b}$ was purified by flash chromatography on a silica gel column eluting with $10 \%$ dichloromethane in hexanes followed by 5\% ethyl acetate in hexanes to give $\mathbf{7 b}(9.7 \mathrm{~g}, 70 \%)$ as a colourless oil. ${ }^{1} \mathbf{H}$ NMR $\left(\mathrm{CDCl}_{3}, 300 \mathrm{MHz}\right): \delta 6.43(\mathrm{~d}, 1 \mathrm{H}, J=2.2 \mathrm{~Hz}), 5.33(\mathrm{~d}, 1 \mathrm{H}, J=5.5 \mathrm{~Hz}), 5.00$ (dd, $1 \mathrm{H}, J=9.9$ and $2.2 \mathrm{~Hz}$ ), 4.96 (s, 1H), 4.82 (s, 1H), 3.97 (dq, 1H, $J=9.3$ and $7.2 \mathrm{~Hz}$ ), 3.61 (dq, 
$1 \mathrm{H}, J=9.3$ and $7.2 \mathrm{~Hz}$ ), 3.32 (t, $1 \mathrm{H}, J=5.5 \mathrm{~Hz}$ ), 2.87 (tdd, $1 \mathrm{H}, J=11.5,5.5$ and $1.6 \mathrm{~Hz}$ ), 2.21 (ddd, $1 \mathrm{H}, J=12.6,5.5$ and 2.2 Hz), 2.18 (s, 3H), 1.87 (s, 3H), 1.61-1.51 (m, 1H), 1.42-1.31 (td, 1H, $J=12.6$ and $9.9 \mathrm{~Hz}$ ), $1.26(\mathrm{t}, 3 \mathrm{H}, J=7.2 \mathrm{~Hz}), 1.03$ (d, 3H, $J=7.2 \mathrm{~Hz}$ ). IR (neat, $\mathrm{cm}^{-1}$ ): 3076, 2973, 2920, 2878, 1710, 1626. LRMS ( $\mathrm{m} / \mathrm{z}$, relative intensity): 276 ( $\left.\mathbf{M}^{+}, 20\right), 233$ (50), 187 (100), 159 (80). HRMS calcd for $\mathrm{C}_{17} \mathrm{H}_{24} \mathrm{O}_{3}$ : 276.1725, found: 276.1734.

\section{Triesters 19a and 20a}

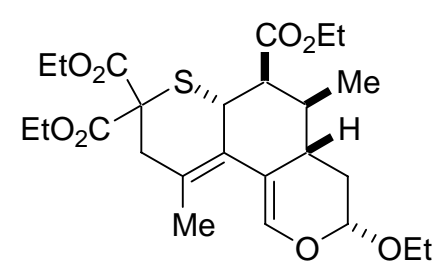

19a

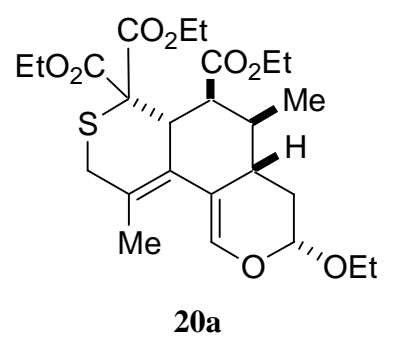

20a

Triene 7a (306 mg, $1.0 \mathrm{mmol})$ was dissolved in acetonitrile $(5.0 \mathrm{~mL})$. Triethylamine (348 $\mu \mathrm{L}, 2.5$ $\mathrm{mmol}$ ) and sulfur powder (115 mg, $3.5 \mathrm{mmol})$ were added at rt. A solution of diethylbromomalonate (available from Aldrich, $341 \mu \mathrm{L}, 2.0 \mathrm{mmol})$ in acetonitrile $(5.0 \mathrm{~mL}$ ) was added over a period of $3 \mathrm{~h}$ with a syringe pump. The reaction mixture was stirred at $\mathrm{rt}$ for $20 \mathrm{~h}$, filtered on celite and concentrated under reduced pressure to give a yellow solid. The crude mixture (1:2 of 19a/20a) was purified by flash chromatography on a silica gel column eluting with 10\% ethyl acetate in hexanes to give 19a (113 mg, $23 \%$ or $29 \%$ based on recovered starting material) as a white solid and 20 a (200 mg, $40 \%$ or $52 \%$ based on recovered starting material) as a white solid.

19a: ${ }^{1} \mathbf{H}$ NMR $\left(\mathrm{CDCl}_{3}, 300 \mathrm{MHz}\right): \delta 6.12$ (d, 1H, $\left.J=2.2 \mathrm{~Hz}\right), 5.04$ (dd, $1 \mathrm{H}, \mathrm{J}=8.8$ and $\left.2.2 \mathrm{~Hz}\right)$, 4.29$4.10(\mathrm{~m}, 6 \mathrm{H}), 3.96-3.86(\mathrm{~m}, 2 \mathrm{H}), 3.56(\mathrm{dq}, 1 \mathrm{H}, J=9.3$ and $7.2 \mathrm{~Hz}), 3.04$ (d, $1 \mathrm{H}, J=14.8 \mathrm{~Hz}), 2.65$ (d, $1 \mathrm{H}, J=14.8 \mathrm{~Hz}$ ), 2.58 (dd, $1 \mathrm{H}, J=11.8$ and $4.1 \mathrm{~Hz}$ ), 2.28-2.23 (m, 1H), 2.12-2.00 (m, 2H), 1.95 (s, 3H), 1.79-1.67 (m, 1H), 1.26-1.21 (m, 12H), 0.91 (d, 3H, $\left.J=7.2 \mathrm{~Hz}) .{ }^{13} \mathbf{C ~ N M R ~ ( C D C l} 3,75.5 \mathrm{MHz}\right): \delta$ 173.9 (s), 168.1 (s), 167.4 (s), 139.6 (d), 127.6 (s), 124.2 (s), 115.2 (s), 100.2 (d), 64.5 (t), 62.3 (t), 62.1 $(\mathrm{t}), 61.8(\mathrm{~s}), 60.2(\mathrm{t}), 49.5(\mathrm{~d}), 40.1(\mathrm{~d}), 35.0(\mathrm{t}), 32.5(\mathrm{~d}), 31.2(\mathrm{t}), 21.9(\mathrm{q}), 15.1(\mathrm{q}), 14.4(\mathrm{q}), 14.0(\mathrm{q})$, 13.6 (q). IR (neat, $\mathrm{cm}^{-1}$ ): 2979, 2947, 2895, 1734, 1717. LRMS ( $\mathrm{m} / \mathrm{z}$, relative intensity): $496\left(\mathrm{M}^{+}, 85\right)$, 450 (50), 377 (50), 351 (50), 277 (100), 259 (50). HRMS calcd for $\mathrm{C}_{25} \mathrm{H}_{36} \mathrm{O}_{8}$ S: 496.2131, found: 496.2134. m.p. ( $\left.{ }^{\circ} \mathrm{C}\right): 156-157$.

20a: ${ }^{1} \mathbf{H}$ NMR $\left(\mathrm{CDCl}_{3}, 300 \mathrm{MHz}\right): \delta 6.30$ (d, $\left.1 \mathrm{H}, J=2.2 \mathrm{~Hz}\right), 5.11$ (dd, $1 \mathrm{H}, J=9.3$ and $\left.2.8 \mathrm{~Hz}\right), 4.32-$ 4.09 (m, 4H), 4.05-3.90 (m, 3H), 3.58 (dq, 1H, $J=9.3$ and 7.2 Hz), 3.58-3.52 (m, 1H), 3.25 (d, $1 \mathrm{H}, J=$ $17.1 \mathrm{~Hz}$ ), 2.98 (dd, 1H, $J=7.7$ and $7.2 \mathrm{~Hz}$ ), 2.88-2.77 (m, 1H), 2.74 (d, $1 \mathrm{H}, J=17.1 \mathrm{~Hz}), 2.05-1.97$ (dm, 1H, $J=12.6 \mathrm{~Hz}$ ), 1.92 (s, 3H), 1.67-1.58 (m, 1H), 1.47-1.35 (td, 1H, J = 12.6 and 9.3 Hz), 1.30$1.21(\mathrm{~m}, 12 \mathrm{H}), 0.86(\mathrm{~d}, 3 \mathrm{H}, J=7.2 \mathrm{~Hz}) .{ }^{13} \mathrm{C}$ NMR $\left(\mathrm{CDCl}_{3}, 75.5 \mathrm{MHz}\right): \delta 173.9(\mathrm{~s}), 168.1(\mathrm{~s}), 167.4$ (s), 139.6 (d), 127.6 (s), 124.2 (s) 115.2 (s), 100.2 (d), $64.5(\mathrm{t}), 62.3$ (t), 62.1 (t), $61.8(\mathrm{~s}), 60.2$ (t), 49.5 (d), 40.1 (d), 35.0 (d), 32.5 (d) 31.3 (t), 21.9 (q), 15.1 (q), 14.4 (q), 14.0 (q), 13.6 (q). IR (neat, cm $\left.{ }^{-1}\right)$ : 2984, 2943, 2881, 1737. LRMS (m/z, relative intensity): 496 ( $\left.\mathrm{M}^{+}, 15\right), 450$ (20), 423 (15), 404 (15), 377 (30), 259 (100). HRMS calcd for $\mathrm{C}_{25} \mathrm{H}_{36} \mathrm{O}_{8} \mathrm{~S}: 496.2131$, found: 496.2134. m.p. $\left({ }^{\circ} \mathrm{C}\right): 151-154$. 
Di-tert-butylbromomalonate (32) used to make thione 17.<smiles>CC(C)(C)OC(=O)C(=O)C(Br)C(=O)OC(C)(C)C</smiles>

32

Di-tert-butylmalonate (200 mg, $0.92 \mathrm{mmol})$ was dissolved in THF $(400 \mathrm{~mL})$ at $0^{\circ} \mathrm{C}$. DBU $(138 \mu \mathrm{L}$, $0.92 \mathrm{mmol}$ ) was added at $0^{\circ} \mathrm{C}$. The reaction was warmed up to RT for $1 \mathrm{~h}$, then cooled to $-78^{\circ} \mathrm{C}$ and $\mathrm{CBr}_{4}$ (306 mg, $0.92 \mathrm{mmol}$ ) was added. The mixture was stirred $1.5 \mathrm{~h}$ at $-78^{\circ} \mathrm{C}$ and quenched with saturated aqueous $\mathrm{NH}_{4} \mathrm{Cl}$. The phases were separated and hexanes were added to the organic phase. The organic layer was washed twice with brine. The aqueous layer was extracted twice with dichloromethane. The organic layers were combined, dried over anhydrous magnesium sulfate and concentrated under reduced pressure to give a yellow oil. The crude product was passed thought a silica gel pad eluting with diethyl ether to give di-tert-butylbromomalonate ${ }^{[3]}$ (32) (219 mg, 80\%) as a colourless oil.

\section{Triesters 19b and 20b}

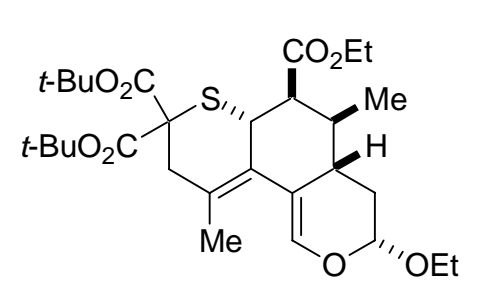

19b

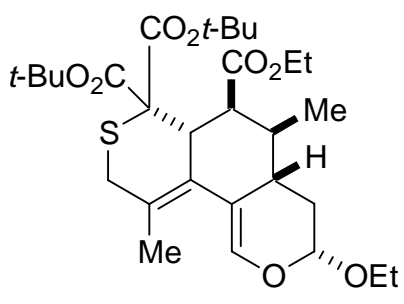

$20 b$

Triene 7a (306 mg, $1.0 \mathrm{mmol})$ was dissolved in acetonitrile $(7.0 \mathrm{~mL})$. Triethylamine $(870 \mu \mathrm{L}, 6.3$ $\mathrm{mmol}$ ) and sulfur powder (280 $\mathrm{mg}, 8.8 \mathrm{mmol}$ ) were added at rt. The reaction mixture was heated to 60 ${ }^{\circ} \mathrm{C}$ and a solution of di-tert-butylbromomalonate (32) $(1.48 \mathrm{~g}, 5.0 \mathrm{mmol})$ in acetonitrile $(7.0 \mathrm{~mL})$ was added over a period of $3 \mathrm{~h}$ with a syringe pump. The reaction mixture was stirred at $60{ }^{\circ} \mathrm{C}$ for $20 \mathrm{~h}$, filtered on celite and concentrated under reduced pressure to give a brown solid. The crude mixture (2:1 of 19b/20b) was purified by flash chromatography on a silica gel column eluting with $10 \%$ ethyl acetate in hexanes to give a 2:1 mixture of $\mathbf{1 9 b}$ and $\mathbf{2 0 b}$ as a colourless oil (507 mg, 92\%). For analytical purposes, $20 \mathrm{mg}$ of this 2:1 mixture was purified by preparative HPLC eluting with $17 \%$ $\mathrm{H}_{2} \mathrm{O}, 50 \%$ acetonitrile and $33 \%$ methanol to afford $7 \mathrm{mg}$ of $\mathbf{1 9 b}$ and $4 \mathrm{mg}$ of $\mathbf{2 0 b}$, both as colourless oil.

19b: ${ }^{1} \mathbf{H}$ NMR $\left(\mathrm{CDCl}_{3}, 300 \mathrm{MHz}\right): \delta 66.14$ (d, 1H, $J=2.2 \mathrm{~Hz}$ ), 5.05 (dd, 1H, $J=9.0$ and $2.2 \mathrm{~Hz}$ ), 4.15 (q, $2 \mathrm{H}, J=7.2 \mathrm{~Hz}$ ), 3.92 (dq, $1 \mathrm{H}, J=9.3$ and $7.2 \mathrm{~Hz}$ ), 3.86 (d, $1 \mathrm{H}, J=11.5 \mathrm{~Hz}$ ), 3.58 (dq, $1 \mathrm{H}, J=9.3$ and $7.2 \mathrm{~Hz}$ ), $2.93(\mathrm{~d}, 1 \mathrm{H}, J=15.4 \mathrm{~Hz}), 2.62(\mathrm{dd}, 1 \mathrm{H}, J=11.5$ and $4.4 \mathrm{~Hz}), 2.57(\mathrm{~d}, 1 \mathrm{H}, J=15.4 \mathrm{~Hz})$, 2.31-2.25 (m, 1H), 2.11-2.00 (m, 2H), 1.97 (s, 3H), 1.78-1.67 (m, 1H), 1.51-1.41 (m, 18H), 1.25 (t, 6H, $J=7.2 \mathrm{~Hz}$ ), 0.91 (d, 3H, $J=7.2 \mathrm{~Hz}$ ). IR (neat, $\mathrm{cm}^{-1}$ ): 2979, 2932, 1738, 1731, 1633, 1576. LRMS (m/z, relative intensity): $552\left(\mathrm{M}^{+}, 25\right), 439$ (20), 393 (25). HRMS calcd for $\mathrm{C}_{29} \mathrm{H}_{44} \mathrm{O}_{8} \mathrm{~S}$ : 552.2757, found: 552.2767 .

20b: ${ }^{1} \mathbf{H}$ NMR $\left(\mathrm{CDCl}_{3}, 300 \mathrm{MHz}\right): \delta 6.28$ (d, 1H, $\left.J=2.3 \mathrm{~Hz}\right), 5.07$ (dd, $1 \mathrm{H}, J=9.9$ and $\left.2.8 \mathrm{~Hz}\right), 4.25-$ 4.10 (m, 1H), 4.05-3.90 (m, 2H), 3.58 (dq, 1H, $J=9.9$ and 7.2Hz), 3.47 (d, 1H, $J=7.2 \mathrm{~Hz}$ ), 3.30 (d, $1 \mathrm{H}, J=17.0 \mathrm{~Hz}$ ), 3.03 (t, 1H, $J=7.2 \mathrm{~Hz}), 2.73$ (d, $1 \mathrm{H}, J=17.0 \mathrm{~Hz}), 2.76-2.65$ (m, 1H), 2.17-1.99 (m, 1H), 1.92 (s, 3H), 1.72-1.60 (m, 1H), 1.55-1.42 (m, 19H), 1.25 (t, 6H, $J=7.2 \mathrm{~Hz}), 0.88$ (d, 3H, $J=7.2$ 
Hz). IR (neat, $\mathrm{cm}^{-1}$ ): 2978, 2932, 2880, 1738, 1731, 1633, 1576. LRMS ( $\mathrm{m} / \mathrm{z}$, relative intensity): 552 $\left(\mathrm{M}^{+}, 25\right), 439$ (20), 393 (25). HRMS calcd for $\mathrm{C}_{29} \mathrm{H}_{44} \mathrm{O}_{8} \mathrm{~S}$ : 552,2757, found: 552.2773.

\section{5-bromo-2,2-dimethyl-[1,3]dioxane-4,6-dione (33) used to make thione 18.}

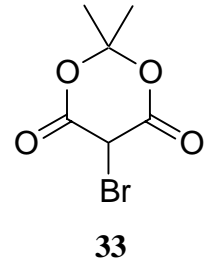

2,2-Dimethyl-[1,3]dioxane-4,6-dione (25.0 g, $174 \mathrm{mmol})$ was dissolved with a $1 \mathrm{M} \mathrm{NaOH}$ solution (174 $\mathrm{mL})$. Bromine $(8.9 \mathrm{~mL}, 174 \mathrm{mmol})$ was added at $0{ }^{\circ} \mathrm{C}$ over a $2 \mathrm{~h}$ period with a syringe pump. The mixture was stirred $30 \mathrm{~min}$ at $0{ }^{\circ} \mathrm{C}$ and filtered. The solid was washed with cold water and dried under vacuum to give 5-bromo-2,2-dimethyl-[1,3]dioxane-4,6-dione (33) (18.0 g, 45\%) as a white solid. ${ }^{\mathbf{1}} \mathbf{H}$ NMR (CDCl $3,300 \mathrm{MHz}): \delta 5.12$ (s, 1H), 1.93 (s, 3H), 1.83 (s, 3H). IR (neat, cm ${ }^{-1}$ ): 3011, 2927, 1783. LRMS ( $\mathrm{m} / \mathrm{z}$, relative intensity): 207((M-CH$\left.)^{+}, 10\right), 178$ (10), 120 (100). HRMS calcd for $\mathrm{C}_{5} \mathrm{H}_{4} \mathrm{BrO}_{4}\left(\left(\mathrm{M}-\mathrm{CH}_{3}\right)^{+}\right)$: 206.9293, found: 206.9296. m.p. $\left({ }^{\circ} \mathrm{C}\right): 53-54$.

\section{Dioxane-4,6-diones 19c and 20c}

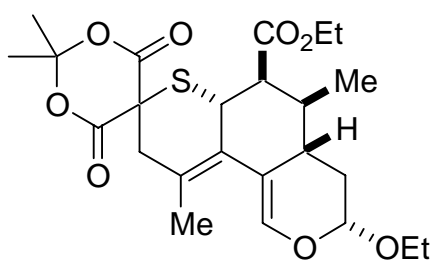

19c

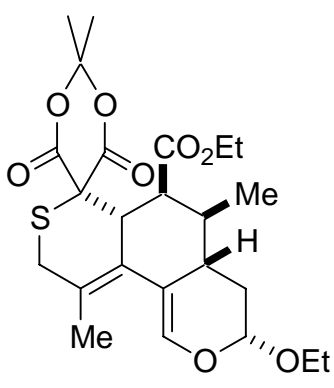

20c

Triene 7a $(8.57 \mathrm{~g}, 28.0 \mathrm{mmol})$ was dissolved in acetonitrile $(140 \mathrm{~mL})$. Triethylamine $(10.7 \mathrm{~mL}, 76.9$ $\mathrm{mmol})$ and sulfur powder $(3.40 \mathrm{~g}, 106 \mathrm{mmol})$ were added at rt. The reaction mixture was heated to 82 ${ }^{\circ} \mathrm{C}$ and a solution of 5-bromo-2,2-dimethyl-[1,3]dioxane-4,6-dione (33) (14.3 g, $61.5 \mathrm{mmol}$ ) in acetonitrile $(140 \mathrm{~mL})$ was added over a period of $3 \mathrm{~h}$ with a syringe pump. The reaction mixture was cooled to rt, filtered on celite and concentrated under reduced pressure to give a brown solid. The crude mixture (14:1 of $\mathbf{1 9 c} / \mathbf{2 0 c}$ ) was purified by flash chromatography on a silica gel column eluting with $10 \%$ ethyl acetate in hexanes to give 19c and 20c as yellowish solids. The solids were separately triturated with vigorous stirring in ethanol for 2 h. The desired white solids 19c (10.1 g, 75\%) and 20c (615 mg, 5\%) were filtered and dried under vacuum pump.

19c: ${ }^{1} \mathbf{H}$ NMR $\left(\mathrm{CDCl}_{3}, 300 \mathrm{MHz}\right): \delta 6.35$ (d, 1H, $\left.J=2.8 \mathrm{~Hz}\right), 5.08$ (dd, 1H, $J=9.3$ and $\left.2.2 \mathrm{~Hz}\right), 4.19$ $4.08(\mathrm{~m}, 2 \mathrm{H}), 4.15$ (d, 1H, $J=11.5 \mathrm{~Hz}), 3.93(\mathrm{dq}, 1 \mathrm{H}, J=9.3$ and $7.2 \mathrm{~Hz}), 3.58(\mathrm{dq}, 1 \mathrm{H}, J=9.3$ and $7.2 \mathrm{~Hz}$ ), 3.09 (d, 1H, $J=14.8 \mathrm{~Hz}$ ), 2.80 (d, 1H, $J=14.8 \mathrm{~Hz}$ ), 2.49 (dd, 1H, $J=11.5$ and $3.3 \mathrm{~Hz}$ ), 2.342.28 (m, 1H), 2.19-2.15 (m, 1H), 2.09 (s, 3H), 2.07-2.00 (m, 1H), 1.85 (s, 3H), 1.81-1.71 (m, $1 \mathrm{H}) 1.71$ (s, 3H), 1.24 (t, 3H, $J=7.2 \mathrm{~Hz}), 1.23$ (t, 3H, $J=7.2 \mathrm{~Hz}), 0.95$ (d, 3H, $J=7.2 \mathrm{~Hz}) .{ }^{13} \mathbf{C ~ N M R}\left(\mathrm{CDCl}_{3}\right.$, $75.5 \mathrm{MHz}$ ): $\delta 171.9$ (s), 167.7 (s), 166.9 (s), 142.6 (d), 130.8 (s), 130.0 (s), 108.1 (s), 105.7 (s), 99.3 (d), 64.2 (t), 60.9 (t), 51.9 (s), 45.0 (d), 41.5 (d), 38.8 (t), 37.2 (d), 36.8 (d), 34.3 (t), 29.4 (q), 29.1 (q), 21.7 (q), 16.8 (q), 15.1 (q), 14.1 (q). IR (neat, $\mathrm{cm}^{-1}$ ): 2978, 2934, 2878, 1777, 1741, 1632. LRMS (m/z, 
relative intensity): 480 ( $\mathrm{M}^{+}$, 80), 434 (30), 348 (60), 322 (100), 259 (60). HRMS calcd for $\mathrm{C}_{24} \mathrm{H}_{32} \mathrm{O}_{8} \mathrm{~S}$ : 480.1818, found: 480.1807 . m.p. $\left({ }^{\circ} \mathrm{C}\right): 158-159$.

20c: ${ }^{1} \mathbf{H}$ NMR $\left(\mathrm{CDCl}_{3}, 300 \mathrm{MHz}\right): \delta 6.30(\mathrm{~d}, 1 \mathrm{H}, J=1.6 \mathrm{~Hz}), 5.04$ (dd, $1 \mathrm{H}, J=8.5$ and $\left.2.2 \mathrm{~Hz}\right), 4.14$ (dq, $1 \mathrm{H}, J=11.0$ and $7.2 \mathrm{~Hz}$ ), 4.04 (dq, $1 \mathrm{H}, J=11.0$ and $7.2 \mathrm{~Hz}$ ), 3.92 (dq, 1H, $J=9.4 \mathrm{and} 7.2 \mathrm{~Hz}$ ), 3.57 (dq, 1H, $J=9.4$ and $7.2 \mathrm{~Hz}$ ), 3.52 (d, 1H, $J=14.8 \mathrm{~Hz}$ ), 3.55-3.51 (m, 1H), 3.05 (d, $1 \mathrm{H}, J=14.8$ Hz), 2.86 (dd, 1H, $J=10.4$ and $3.3 \mathrm{~Hz}$ ), 2.25-2.09 (m, 2H), 2.06 (d, 3H, $J=1.6 \mathrm{~Hz}$ ), 2.08-2.00 (m, $1 \mathrm{H}), 1.88(\mathrm{~s}, 3 \mathrm{H}), 1.78(\mathrm{~s}, 3 \mathrm{H}), 1.75-1.64(\mathrm{~m}, 1 \mathrm{H}), 1.24$ (t, 3H, $J=7.2 \mathrm{~Hz}), 1.22(\mathrm{t}, 3 \mathrm{H}, J=7.2 \mathrm{~Hz})$, 1.00 (d, 3H, $J=7.2 \mathrm{~Hz}$ ). IR (neat, $\mathrm{cm}^{-1}$ ): 2981, 2931, 2878, 1733, 1638. LRMS (m/z, relative intensity): 480 ( $\left.\mathrm{M}^{+}, 25\right), 376$ (10), 348 (25), 322 (30), 260 (50), 187 (60), 159 (100). HRMS calcd for $\mathrm{C}_{24} \mathrm{H}_{32} \mathrm{O}_{8} \mathrm{~S}$ : 480.1818, found: 480.1807 . m.p. $\left({ }^{\circ} \mathrm{C}\right): 139-140$.

\section{5-(phtalimide- $N$-sulfenyl)-2,2-dimethyl-[1,3]dioxane-4,6-dione (34) used to make thione 18.}<smiles>CC1(C)OC(=O)C(SN2C(=O)c3ccccc3C2=O)=C(O)O1</smiles>

Phtalimide sulfenyl chloride ${ }^{[4]}(1.72 \mathrm{~g}, 8.1 \mathrm{mmol})$ was dissolved in THF $(10.0 \mathrm{~mL})$ and the solution was cooled to $-10{ }^{\circ} \mathrm{C}$. A solution of 2,2-dimethyl-[1,3]dioxane-4,6-dione (700 mg, $4.9 \mathrm{mmol}$ ) in THF $(5.0 \mathrm{~mL})$ was added over a 15 min period. The mixture was stirred $1 \mathrm{~h}$ at $0{ }^{\circ} \mathrm{C}$. The solid was filtered, washed with cold THF and dried under vacuum to give 34 (1.44 g, 92\%) as a white solid. ${ }^{\mathbf{1}} \mathbf{H}$ NMR $\left(\mathrm{CDCl}_{3}, 300 \mathrm{MHz}\right): \delta$ 7.95-7.92 (m, 2H), 7.85-7.80 (m, 2H), $1.86(\mathrm{~s}, 6 \mathrm{H}) .{ }^{13} \mathrm{C}$ NMR $\left(\mathrm{CDCl}_{3}, 75.5\right.$ MHz): $\delta 166.4$ (s), 161.0 (s), 135.2 (d), 134.3 (s), 131.6 (s), 124.5 (d), 124.3 (s), 123.6 (s), 107.7 (s), 58.5 (s), 28.3 (q). IR (neat, $\mathrm{cm}^{-1}$ ): 3202, 3061, 1744, 1714. m.p. $\left({ }^{\circ} \mathrm{C}\right): 189-190$.

\section{Dioxane-4,6-diones 21 and 22}

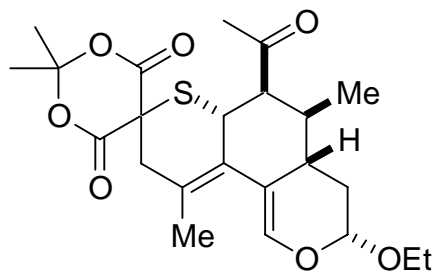

21

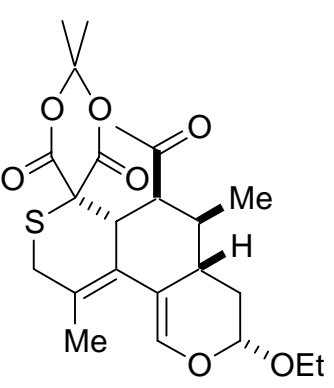

22

Triene 7b (2.59 g, $9.4 \mathrm{mmol}$ ) was dissolved in dichloromethane (95 mL). 5-(phtalimide-N-sulfenyl)2,2-dimethyl-[1,3]dioxane-4,6-dione (34) (3.02 g, $9.4 \mathrm{mmol}$ ) was added and the mixture was cooled to $-10{ }^{\circ} \mathrm{C}$. Pyridine $(0.83 \mathrm{~mL}, 10.3 \mathrm{mmol})$ was added and the reaction mixture was stirred $10 \mathrm{~h}$ at $-10{ }^{\circ} \mathrm{C}$. The reaction mixture was quenched with saturated aqueous $\mathrm{NH}_{4} \mathrm{Cl}$. The phases were separated and the aqueous phase was extracted three times with dichloromethane. The organic layers were combined, washed once with water, washed once with brine, dried over anhydrous magnesium sulfate and concentrated under reduced pressure to give a yellowish solid. The crude mixture (30:1 of 21/22) was 
purified by flash chromatography on a silica gel column eluting with $25 \%$ ethyl acetate in hexanes to give 21 (2.57 g, 61\%) and 22 (84 mg, 2\%) as white solids.

21: ${ }^{1} \mathbf{H}$ NMR $\left(\mathrm{CDCl}_{3}, 300 \mathrm{MHz}\right): \delta 6.26(\mathrm{~s}, 1 \mathrm{H}), 4.89$ (dd, $1 \mathrm{H}, J=8.8$ and $\left.1.7 \mathrm{~Hz}\right), 3.96(\mathrm{dq}, 1 \mathrm{H}, J=$ 9.3 and $7.2 \mathrm{~Hz}), 3.73(\mathrm{dm}, 1 \mathrm{H}, J=11.3 \mathrm{~Hz}), 3.60(\mathrm{dq}, 1 \mathrm{H}, J=9.3$ and $7.2 \mathrm{~Hz}), 3.03(\mathrm{~d}, 1 \mathrm{H}, J=17.6$ $\mathrm{Hz}), 2.77$ (d, 1H, $J=17.6 \mathrm{~Hz}$ ), 2.59 (t, 1H, $J=11.3 \mathrm{~Hz}$ ), 2.27-2.19 (m, 1H), 2.18 (s, 3H), 2.14-1.99 (m, 1H), 1.96 (s, 3H), $1.92(\mathrm{~s}, 3 \mathrm{H}), 1.74(\mathrm{~s}, 3 \mathrm{H}), 1.70-1.63(\mathrm{~m}, 1 \mathrm{H}), 1.59-1.49(\mathrm{~m}, 1 \mathrm{H}), 1.26$ (t, 3H, $J=$ $7.2 \mathrm{~Hz}), 0.90$ (d, 3H, J= 6.6 Hz). ${ }^{13} \mathrm{C}$ NMR $\left(\mathrm{CDCl}_{3}, 75.5 \mathrm{MHz}\right): \delta 206.5$ (s), 167.5 (s), 166.9 (s), 142.4 (d), 131.0 (s), 130.1 (s), 108.3 (s), 105.7 (s), 98.9 (d), 64.3 (t), 52.0 (d), 52.0 (s), 40.4 (d), 38.8 (t), 37.3 (d), 36.3 (t), 36.2 (d), 34.4 (q), 29.4 (q), 29.1 (q), 21.7 (q), 16.4 (q) 15.2 (q). IR (neat, $\mathrm{cm}^{-1}$ ): 2971, 2923, 2875, 1772, 1748, 1739. LRMS ( $\mathrm{m} / \mathrm{z}$, relative intensity): $450\left(\mathrm{M}^{+}, 60\right), 404$ (30), 318 (40), 233 (80), 187 (100). HRMS calcd for $\mathrm{C}_{23} \mathrm{H}_{30} \mathrm{O}_{7} \mathrm{~S}: 450.1712$, found: 450.1703 . m.p. $\left({ }^{\circ} \mathrm{C}\right): 179-180$.

22: ${ }^{1} \mathbf{H}$ NMR $\left(\mathrm{CDCl}_{3}, 300 \mathrm{MHz}\right): \delta 6.26(\mathrm{~s}, 1 \mathrm{H}), 4.89$ (dd, $1 \mathrm{H}, J=8.8$ and $\left.1.7 \mathrm{~Hz}\right), 3.96(\mathrm{dq}, 1 \mathrm{H}, J=$ 9.3 and 7.2 Hz), 3.75-3.70 (m, 1H), $3.60(\mathrm{dq}, 1 \mathrm{H}, J=9.3$ and $7.2 \mathrm{~Hz}$ ), $3.03(\mathrm{~d}, 1 \mathrm{H}, J=17.6 \mathrm{~Hz}), 2.77$ (d, $1 \mathrm{H}, J=17.6 \mathrm{~Hz}), 2.59(\mathrm{t}, 1 \mathrm{H}, J=11.3 \mathrm{~Hz}), 2.27-2.19(\mathrm{~m}, 1 \mathrm{H}), 2.18(\mathrm{~s}, 3 \mathrm{H}), 2.14-1.99(\mathrm{~m}, 1 \mathrm{H})$, 1.96 (s, 3H), 1.92 (s, 3H), 1.74 (s, 3H), 1.70-1.63 (m, 1H), 1.59-1.49 (m, 1H), 1.26 (t, 3H, $J=7.2 \mathrm{~Hz}$ ), 0.90 (d, 3H, J=6.6 Hz). ${ }^{13}$ C NMR $\left(\mathrm{CDCl}_{3}, 75.5 \mathrm{MHz}\right): \delta 209.9$ (s), 165.6 (s), 163.8 (s), 140.9 (d), 126.5 (s), 124.9 (s), 112.4 (s), 106.4 (s), 99.4 (d), 64.6 (t), 60.9 (d), 45.0 (s), 44.5 (d), 42.1 (d), 38.6 (d), $36.7(\mathrm{t}), 34.0$ (t), 31.8 (q), 29.4 (q), 27.5 (q), 22.5 (q), 16.8 (q), 15.1 (q). IR (neat, $\mathrm{cm}^{-1}$ ): 2977, 2924, 2882, 1772, 1733, 1699. LRMS ( $\mathrm{m} / \mathrm{z}$, relative intensity): 450 ( $\left.\mathrm{M}^{+}, 65\right), 404$ (65), 346 (70), 318 (90), 249 (60), 84 (100). HRMS calcd for $\mathrm{C}_{23} \mathrm{H}_{30} \mathrm{O}_{7} \mathrm{~S}: 450.1712$, found: 450.1721 . m.p. $\left({ }^{\circ} \mathrm{C}\right): 164-165$.

\section{Ester 35}

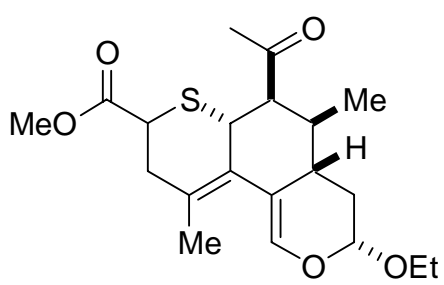

35

Dioxane-4,6-dione 21 (794 mg, $1.76 \mathrm{mmol})$ was suspended in methanol $(10.0 \mathrm{~mL})$. Ni(acac) 2 (135 mg, $0.5 \mathrm{mmol}$ ) was added at rt. The reaction mixture was heated to $65^{\circ} \mathrm{C} 15 \mathrm{~h}$, cooled to rt and quenched with water. The phases were separated and the aqueous phase was extracted three times with dichloromethane. The organic layers were combined, washed once with water, washed once with brine, dried over anhydrous magnesium sulfate and concentrated under reduced pressure. The crude product 35 (655 mg, 98\%, 3:2 diasteromer mixture as a white gum) was used without purification for the next reaction. A small amount of $\mathbf{3 5}$ was purified by flash chromatography on a silica gel column eluting with $15 \%$ ethyl acetate in hexanes for analytical purposes.

Minor diastereomer of 35: ${ }^{1} \mathbf{H}$ NMR $\left(\mathrm{CDCl}_{3}, 300 \mathrm{MHz}\right): \delta 6.18(\mathrm{~d}, 1 \mathrm{H}, J=2.2 \mathrm{~Hz}), 5.07$ (dd, $1 \mathrm{H}, J=$ 8.5 and $2.5 \mathrm{~Hz}$ ), 4.05 (dd, 1H, $J=5.5$ and $2.8 \mathrm{~Hz}$ ), 3.93 (dq, 1H, $J=9.3$ and $7.2 \mathrm{~Hz}), 3.80$ (d, 1H, $J=$ $11.0 \mathrm{~Hz}$ ), 3.64 (s, 3H), 3.57 (dq, $1 \mathrm{H}, J=9.3$ and $7.2 \mathrm{~Hz}$ ), 2.79-2.73 (m, 2H), 2.37 (dd, $1 \mathrm{H}, J=14.9$ and $5.5 \mathrm{~Hz}), 2.29-2.22(\mathrm{~m}, 1 \mathrm{H}), 2.14(\mathrm{~s}, 3 \mathrm{H}), 2.16-2.01(\mathrm{~m}, 2 \mathrm{H}), 2.01(\mathrm{~s}, 3 \mathrm{H}), 1.81-1.70(\mathrm{~m}, 1 \mathrm{H}) 1.24(\mathrm{t}$, $3 \mathrm{H}, J=7.2 \mathrm{~Hz}), 0.82(\mathrm{~d}, 3 \mathrm{H}, J=7.7 \mathrm{~Hz}) .{ }^{13} \mathrm{C} \mathrm{NMR}\left(\mathrm{CDCl}_{3}, 75.5 \mathrm{MHz}\right): \delta 208.1$ (s), 173.1 (s), 141.8 (d), 130.4 (s), 130.1 (s), 109.1 (s), 98.8 (d), 64.2 (t), 53.6 (d), 52.2 (q), 43.9 (d), 37.2 (d), 36.7 (d), 35.8 (d), 34.3 (t), 33.7 (t), 29.8 (q), 21.9 (q), 16.2 (q), 15.2 (q). IR (neat, $\mathrm{cm}^{-1}$ ): 2977, 2924, 2882, 1737, 
1710, 1630. LRMS (m/z, relative intensity): 380 ( $\left.\mathrm{M}^{+}, 50\right), 334$ (60), 291 (60), 205 (100). HRMS calcd for $\mathrm{C}_{20} \mathrm{H}_{28} \mathrm{O}_{5} \mathrm{~S}: 380.1657$, found: 380.1649 .

Major diastereomer of 35: ${ }^{1} \mathbf{H}$ NMR $\left(\mathrm{CDCl}_{3}, 300 \mathrm{MHz}\right): \delta 6.22(\mathrm{~d}, 1 \mathrm{H}, J=2.8 \mathrm{~Hz}), 5.07$ (dd, $1 \mathrm{H}, J=$ 8.8 and $2.8 \mathrm{~Hz}), 3.94(\mathrm{dq}, 1 \mathrm{H}, J=9.3$ and $7.2 \mathrm{~Hz}), 3.94-3.89(\mathrm{~m}, 1 \mathrm{H}), 3.73(\mathrm{~s}, 3 \mathrm{H}), 3.60(\mathrm{dq}, 1 \mathrm{H}, J=$ 9.4 and $7.2 \mathrm{~Hz}$ ), 3.58-3.48 (m, $1 \mathrm{H}), 2.79(\mathrm{dd}, 1 \mathrm{H}, J=11.6$ and $4.4 \mathrm{~Hz}), 2.58-2.55(\mathrm{~m}, 2 \mathrm{H}), 2.38-2.29$ (m, 1H), 2.17 (s, 3H), 2.11-1.96 (m, 2H), 1.96 (s, 3H), 1.76-1.65 (m, 1H), 1.25 (t, 3H, J = 7.2 Hz), 0.84 $(\mathrm{d}, 3 \mathrm{H}, J=7.7 \mathrm{~Hz}) .{ }^{13} \mathrm{C}$ NMR $\left(\mathrm{CDCl}_{3}, 75.5 \mathrm{MHz}\right): \delta 208.5$ (s), 172.6 (s), 141.0 (d), 130.6 (s), 129.1 (s), 110.2 (s), 99.0 (d), 64.3 (t), 54.4 (d), 52.5 (q), 42.9 (d), 37.5 (d), 36.6 (d), 35.9 (d), 34.6 (t), 34.0 (t), 30.8 (q), 21.2 (q), 16.0 (q), 15.2 (q). IR (neat, $\mathrm{cm}^{-1}$ ): 2969, 2931, 2874, 1734, 1699, 1652. LRMS ( $\mathrm{m} / \mathrm{z}$, relative intensity): 380 ( $\left.\mathrm{M}^{+}, 60\right), 334$ (50), 291 (70), 266 (60), 205 (100). HRMS calcd for $\mathrm{C}_{20} \mathrm{H}_{28} \mathrm{O}_{5} \mathrm{~S}$ : 380.1657, found: 380.1649 .

\section{Aldehyde 24}

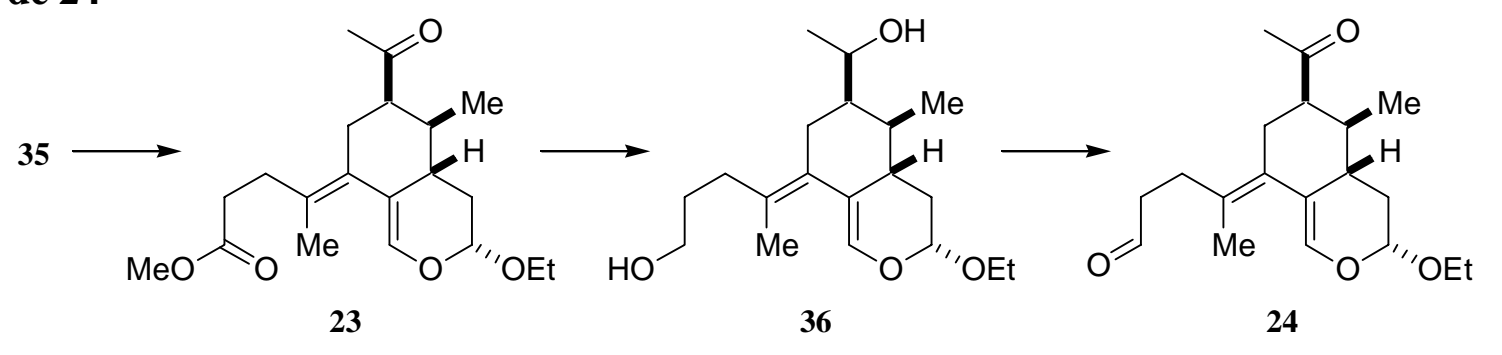

Ester 35 (677 mg, $1.78 \mathrm{mmol}$ ) was disolved in THF (8.0 mL). Raney nickel 2800, as a slurry in water, was added at $\mathrm{rt}$ and the reaction mixture was stirred at $\mathrm{rt}$ for $2 \mathrm{~h}$. The reaction mixture was filtered on celite and the phases were separated. The aqueous phase was extracted twice with ethyl acetate. The organic layers were combined, dried over anhydrous magnesium sulfate and concentrated under reduced pressure. Ester 23 (588 mg, 94\%, purity of 65\% by GC/MS) was used without purification for the next reaction.

Crude ester 23 (588 mg, $1.68 \mathrm{mmol})$ was dissolved in THF (18.0 mL) at $-10{ }^{\circ} \mathrm{C}$. Lithium aluminium hydride (191 mg, $5.04 \mathrm{mmol}$ ) was added in four portions during a $15 \mathrm{~min}$ period. The reaction mixture was stirred 30 min at $-10{ }^{\circ} \mathrm{C}$ and quenched slowly with saturated aqueous $\mathrm{NH}_{4} \mathrm{Cl}$. The phases were separated and the aqueous phase was extracted three times with ethyl acetate. The organic layers were combined, washed once with water, washed once with brine, dried over anhydrous magnesium sulfate and concentrated under reduced pressure to give a yellowish oil. The crude product $\mathbf{3 6}$ was purified by flash chromatography on a silica gel column eluting with $30 \%$ to $50 \%$ ethyl acetate in hexanes to give a 8:1 mixture of diastereomeric diols $\mathbf{3 6}$ (288 mg, 50\% for two steps) as a white solid. ${ }^{\mathbf{1}} \mathbf{H} \mathbf{~ N M R}\left(\mathrm{CDCl}_{3}\right.$, $300 \mathrm{MHz}$ ): $\delta 6.07$ (d, $1 \mathrm{H}$ minor diastereomer, $J=2.2 \mathrm{~Hz}$ ), 6.03 (d, major diastereomer, $J=2.2 \mathrm{~Hz}$ ), 4.98 (dd, $1 \mathrm{H}$ major diastereomer, $J=9.3$ and $2.2 \mathrm{~Hz}$ ), 4.91 (dd, $1 \mathrm{H}$, minor diastereomer , $J=9.6$ and 1.9), 3.94 (dq, $1 \mathrm{H}$ major diastereomer, $J=9.3$ and $7.2 \mathrm{~Hz}$ ), 3.92 (dq, $1 \mathrm{H}$ minor diastereomer, $J=9.3$ and 7.2 Hz), 3.73-3.54 (m, 4H), 2.76 (dd, 1H minor diastereomer, $J=15.1$ and $6.3 \mathrm{~Hz}), 2.44-2.34$ (m, 2H minor diastereomer), 2.23-1.89 (m, 6H), 1.82 (d, 3H minor diastereomer, $J=2.2 \mathrm{~Hz}$ ), $1.79(\mathrm{~s}, 3 \mathrm{H}$ major diastereomer), 1.72-1.60 (m, 4H), 1.55-1.46 (m, 1H), 1.25 (t, 3H, $J=7.2 \mathrm{~Hz}), 1.19(\mathrm{~d}, 3 \mathrm{H}, J=$ $6.1 \mathrm{~Hz}$ ), 0.98 (d, 3H minor diastereomer, $J=7.2 \mathrm{~Hz}$ ), 0.91 (d, 3H major diastereomer, $J=7.2 \mathrm{~Hz}$ ). IR (neat, $\mathrm{cm}^{-1}$ ): 3538-3129 (br), 2969, 2924, 2870, 1638, 1535. LRMS ( $\mathrm{m} / \mathrm{z}$, relative intensity): 324 (M , 100), 189 (60). HRMS calcd for $\mathrm{C}_{19} \mathrm{H}_{32} \mathrm{O}_{4}$ : 324.2300, found: 324.2306. m.p. ( $\left.{ }^{\circ} \mathrm{C}\right)$ : 99-100.

The 8:1 mixture of diols 36 (52 $\mathrm{mg}, 0.160 \mathrm{mmol}$ ) was dissolved in ethyl acetate (3.0 mL) and IBX (258 $\mathrm{mg}, 0.92 \mathrm{mmol}$ ) was added. The reaction mixture was stirred $2 \mathrm{~h}$ at $77^{\circ} \mathrm{C}$. The solution was cooled to 0 
${ }^{\circ} \mathrm{C}$, filtered and evaporated under reduced pressure. The crude product 24 was purified by flash chromatography on a silica gel column eluting with 30\% ethyl acetate in hexanes to give 24 (50 mg, 98\%) as a colourless oil. ${ }^{1} \mathbf{H}$ NMR $\left(\mathrm{CDCl}_{3}, 300 \mathrm{MHz}\right): \delta 9.78(\mathrm{~s}, 1 \mathrm{H}), 6.06$ (d, $\left.1 \mathrm{H}, J=1.6 \mathrm{~Hz}\right), 4.91$ (dd, $1 \mathrm{H}, J=9.1$ and $1.9 \mathrm{~Hz}$ ), $3.95(\mathrm{dq}, 1 \mathrm{H}, J=9.3$ and $7.2 \mathrm{~Hz}$ ), 3.59 (dq, $1 \mathrm{H}, J=9.3$ and $7.2 \mathrm{~Hz}$ ), 2.85-2.75 (m, 2H), 2.57-2.32 (m, 4H), 2.31-2.24 (m, 1H), 2.20-2.11 (m, 2H), 2.12 (s, 3H), 1.78 (d, 3H, $J=1.6 \mathrm{~Hz}), 1.72-1.65(\mathrm{~m}, 1 \mathrm{H}), 1.52-1.41(\mathrm{~m}, 1 \mathrm{H}), 1.25(\mathrm{t}, 3 \mathrm{H}, J=7.2 \mathrm{~Hz}), 0.92$ (d, 3H, $J=6.6 \mathrm{~Hz})$. ${ }^{13}$ C NMR (CDCl, 75.5 MHz): $\delta 210.2$ (s), 202.1 (d), 139.2 (d), 128.0 (s), 127.5 (s), 114.5 (s), 99.4 (d), $64.4(\mathrm{t}), 52.2(\mathrm{~d}), 42.5(\mathrm{t}), 39.1(\mathrm{~d}), 36.2(\mathrm{~d}), 35.3(\mathrm{t}), 30.3(\mathrm{q}), 30.2(\mathrm{t}), 26.2(\mathrm{t}), 19.9(\mathrm{q}), 16.6(\mathrm{q}), 15.2$ (q). IR (neat, $\mathrm{cm}^{-1}$ ): 3057, 2969, 2916, 2874, 2721, 1710, 1638. LRMS ( $\mathrm{m} / \mathrm{z}$, relative intensity): 320 ( $\mathrm{M}^{+}$, 40), 274 (60), 231 (100), 205 (60). HRMS calcd for $\mathrm{C}_{19} \mathrm{H}_{28} \mathrm{O}_{4}$ : 320.1987, found: 320.1993.

\section{$\alpha, \beta$-Unsaturated esters 26a and 26b}
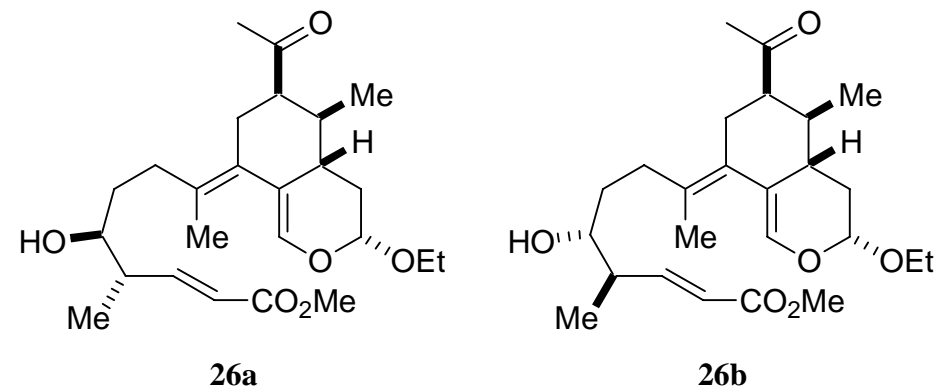

To a stirred solution $\left(-78^{\circ} \mathrm{C}\right)$ of aldehyde $24(50 \mathrm{mg}, 0.16 \mathrm{mmol})$ in diethyl ether $(2.0 \mathrm{~mL})$ was added tris(pentafluorophenyl)borane $\left(80 \mathrm{mg}, 0.16 \mathrm{mmol}\right.$ ). A solution of ketene acetal $25^{[5]}$ (76 $\mathrm{mg}, 0.31$ $\mathrm{mmol})$ and 2-propanol $(13 \mu \mathrm{L}, 0.17 \mathrm{mmol})$ in diethyl ether $(1.0 \mathrm{~mL})$ was added over a period of $6 \mathrm{~h}$ at $78{ }^{\circ} \mathrm{C}$. Triethylamine $(24 \mu \mathrm{L}, 0.17 \mathrm{mmol})$ and silica gel $(500 \mathrm{mg}$ ) were successively added to the reaction mixture. The mixture was evaporated under reduced pressure and flash chromatography eluting with 30\% ethyl acetate in hexanes afford a $1: 1$ mixture of $\mathbf{2 6 a}$ and $\mathbf{2 6 b}$ (42 $\mathrm{mg}, 62 \%$ ) as a colourless oil. ${ }^{1} \mathbf{H}$ NMR $\left(\mathrm{CDCl}_{3}, 300 \mathrm{MHz}\right): \delta 6.94$ (dd, $1 \mathrm{H}, J=15.9$ and $\left.7.7 \mathrm{~Hz}\right), 6.05$ (s, $\left.1 \mathrm{H}\right), 5.87$ (d, $1 \mathrm{H}, J=15.9 \mathrm{~Hz}$ ), $4.91(\mathrm{~d}, 1 \mathrm{H}, J=7.7 \mathrm{~Hz}), 3.96(\mathrm{dq}, 1 \mathrm{H}, J=9.3$ and $7.2 \mathrm{~Hz}), 3.73(\mathrm{~s}, 3 \mathrm{H}), 3.60$ (dq, $1 \mathrm{H}, J=9.3$ and $7.2 \mathrm{~Hz}), 3.54-3.48(\mathrm{~m}, 1 \mathrm{H}), 2.89-2.78(\mathrm{~m}, 2 \mathrm{H}), 2.62-2.51(\mathrm{~m}, 1 \mathrm{H}), 2.45-2.38(\mathrm{~m}, 1 \mathrm{H})$, 2.34-1.97 (m 4H), 2.12 (s, 3H), 1.79 (d, 3H diastereomer A, $J=2.2 \mathrm{~Hz}$ ), 1.77 (d, 3H diastereomer B, $J$ $=1.1 \mathrm{~Hz}), 1.69-1.32(\mathrm{~m}, 3 \mathrm{H}), 1.26(\mathrm{t}, 3 \mathrm{H}, J=7.2 \mathrm{~Hz}), 1.09(\mathrm{~d}, 3 \mathrm{H}$ diastereomer $\mathrm{A}, J=6.6 \mathrm{~Hz}), 1.08$ (d, $3 \mathrm{H}$ diastereomer $\mathrm{B}, J=6.6 \mathrm{~Hz}$ ), 0.94 (d, 3H diastereomer $\mathrm{B}, J=6.6 \mathrm{~Hz}), 0.93$ (d, 3H diastereomer A, $J=7.2 \mathrm{~Hz}$ ). IR (neat, $\mathrm{cm}^{-1}$ ): 3652-3271 (br), 2973, 2920, 2870, 1706, 1638. LRMS ( $\mathrm{m} / \mathrm{z}$, relative intensity): 434 (M+, 35), 345 (55), 159 (95), 145 (80), 114 (100). HRMS calcd for $\mathrm{C}_{25} \mathrm{H}_{38} \mathrm{O}_{6}$ : 434.2668, found: 434.2659 .

\section{$\alpha, \beta$-Unsaturated esters 27a and 27b}
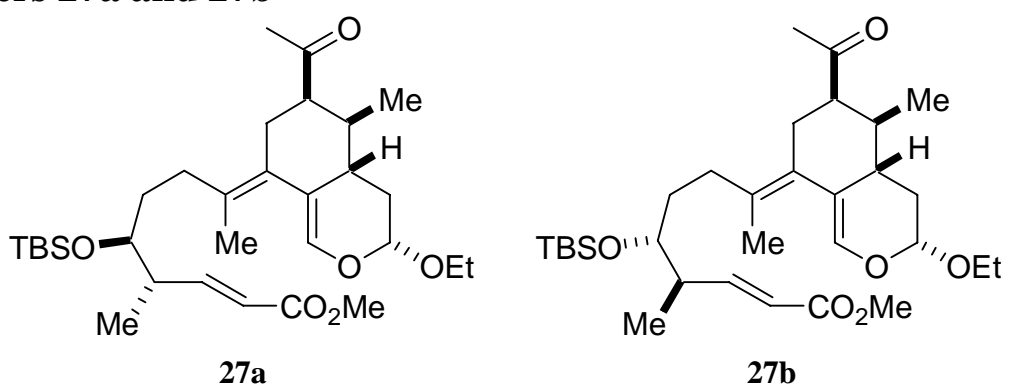

To a stirred solution of alcohol 26a and $26 \mathbf{b}(120 \mathrm{mg}, 0.27 \mathrm{mmol})$ in DMF (1.5 mL) was added imidazole (50 mg, $0.73 \mathrm{mmol}$ ) and tert-butyldimethylsilyl chloride ( $87 \mathrm{mg}, 0.58 \mathrm{mmol}$ ). The reaction 
mixture was stirred at rt for 3 days and quenched with saturated aqueous $\mathrm{NH}_{4} \mathrm{Cl}$. The phases were separated and the aqueous phase was extracted twice with ethyl acetate. The organic layers were combined, washed four times with water, washed once with brine, dried over anhydrous magnesium sulfate and concentrated under reduced pressure. The crude products were purified by flash chromatography on a silica gel column eluting with $10 \%$ ethyl acetate in hexanes to give a 1:1 mixture of 27a and 27b (124 mg, 81\%) as a colorless oil. ${ }^{\mathbf{1}} \mathbf{H} \mathbf{~ N M R}\left(\mathrm{CDCl}_{3}, 300 \mathrm{MHz}\right): \delta 7.02(\mathrm{dd}, 1 \mathrm{H}$ diastereomer A, $J=15.9$ and $7.2 \mathrm{~Hz}$ ), 7.00 (dd, $1 \mathrm{H}$ diastereomer B, $J=15.9$ and 7.2 Hz), 6.05-6.04 (m, 1H), 5.83 (d, 1H, $J=15.9 \mathrm{~Hz}), 4.92-4.88$ (m, 1H), $3.96(\mathrm{dq}, 1 \mathrm{H}, J=9.3$ and $7.2 \mathrm{~Hz}$ ), 3.73 (s, $3 \mathrm{H}$ diastereomer A), 3.72 (s, 3H diastereomer B), $3.60(\mathrm{dq}, 1 \mathrm{H}, J=9.3$ and $7.2 \mathrm{~Hz}), 3.60-3.55(\mathrm{~m}, 1 \mathrm{H})$, 2.81-2.72 (m, 2H), 2.64-2.45 (m, 2H), 2.31-2.05 (m, 3H), 2.10 (s, 3H), 1.97-1.79 (m, 1H), 1.76-1.75 (m, 3H), 1.70-1.56 (m, 1H), 1.50-1.33 (m, 3H), 1.26 (t, 3H, $J=7.2 \mathrm{~Hz}), 1.03$ (d, 3H, $J=6.6 \mathrm{~Hz}), 0.93$ (d, 3H, $J=7.2 \mathrm{~Hz}$ ), 0.90 (s, 9H), 0.05 (s, 6H diastereomer A), 0.04 (s, 6H diastereomer B). ${ }^{13} \mathbf{C}$ NMR $\left(\mathrm{CDCl}_{3}, 75.5 \mathrm{MHz}\right.$ ): $\delta 210.0$ (s), 167.0 (s), 151.9 (d, diastereomer A), 151.6 (d, diastereomer B), 139.0 (d), 130.2 (s), 126.0 (s), 120.6 (d, diastereomer B), 120.5 (d, diastereomer A), 114.8 (s), 99.5 (d), 75.5 (d, diastereomer B), 75.3 (d, diastereomer A), 64.4 (t), 52.5 (d, diastereomer A), 52.4 (d, diastereomer B), 51.4 (q), 41.9 (d, diastereomer B), 41.8 (d, diastereomer A), 39.5 (d, diastereomer B) 39.2 (d, diastereomer A), 36.2 (d, diastereomer A), 36.1 (d, diastereomer B), 35.5 (t), $32.4(\mathrm{t}), 30.6(\mathrm{t}$, diastereomer A), 30.4 (t, diastereomer B), 30.0 (t), 25.8 (q), 20.3 (q), 18.1 (s), 16.7 (q), 15.2 (q), 14.6 (q), 14.3 (q), -4.3 (q), -4.5 (q). IR (neat, $\mathrm{cm}^{-1}$ ): 2958, 2935, 2889, 2858, 1718, 1645, 1523. LRMS (m/z, relative intensity): $548\left(\mathrm{M}^{+}, 40\right), 505$ (45), 459 (50), 257 (100). HRMS calcd for $\mathrm{C}_{31} \mathrm{H}_{52} \mathrm{O}_{6} \mathrm{Si}$ : 548.3533, found: 548.3527.

\section{Tetracyclic compounds $28 \mathrm{a}$ and $28 \mathrm{~b}$}

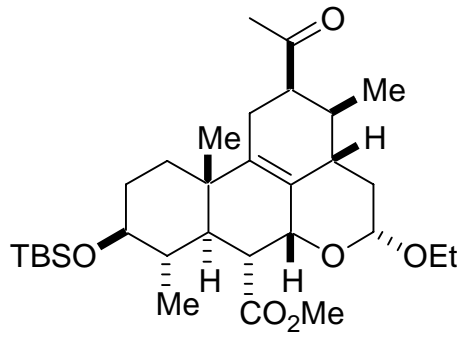

28a

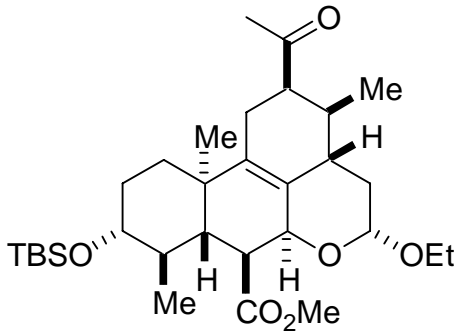

28b

A solution of $27 \mathbf{a}$ and $27 \mathbf{b}(50 \mathrm{mg}, 0.09 \mathrm{mmol})$ in toluene $(4.0 \mathrm{~mL})$ was heated to $295^{\circ} \mathrm{C}$ for $2 \mathrm{~h}$ under $\mathrm{N}_{2}$ in a degassed sealed tube (the tube was previously washed with $2 \mathrm{~N} \mathrm{HCl}, \mathrm{H}_{2} \mathrm{O}, \mathrm{NH}_{4} \mathrm{OH}, \mathrm{H}_{2} \mathrm{O}$ and acetone). After this time, the tube was unsealed and the toluene was evaporated under reduced pressure. The crude products were purified by flash chromatography on a silica gel column eluting with $15 \%$ ethyl acetate in hexanes to give 28a (18 mg, 35 \%) and 28b (16 mg, 33\%) as white solids for a combined yield of $68 \%$.

28a: ${ }^{1} \mathrm{H}$ NMR $\left(\mathrm{CDCl}_{3}, 600 \mathrm{MHz}\right): \delta 4.53(\mathrm{dd}, 1 \mathrm{H}, J=9.5$ and $1.8 \mathrm{~Hz}), 3.88(\mathrm{~d}, 1 \mathrm{H}, J=7.7 \mathrm{~Hz}), 3.81$ (dq, $1 \mathrm{H}, J=9.3$ and $7.2 \mathrm{~Hz}$ ), 3.66 (s, 3H), 3.44 (dq, $1 \mathrm{H}, J=9.3$ and $7.2 \mathrm{~Hz}$ ), 3.22 (td, $1 \mathrm{H}, J=10.3$ and $4.8 \mathrm{~Hz}$ ), 2.91 (dd, $1 \mathrm{H}, J=12.8$ and $7.7 \mathrm{~Hz}$ ), 2.70-2.67 (m,1H,), 2.25 (dd, $1 \mathrm{H}, J=17.9$ and $6.6 \mathrm{~Hz}$ ), 2.19 (dd, $1 \mathrm{H}, J=17.9$ and $5.5 \mathrm{~Hz}$ ), 2.13-2.10 (m, 1H), 2.11 (s, 3H), 1.99 (dm, 1H, $J=12.5 \mathrm{~Hz}), 1.87$ (dd, $1 \mathrm{H}, J=12.8$ and $10.8 \mathrm{~Hz}), 1.82-1.72(\mathrm{~m}, 3 \mathrm{H}), 1.63-1.51(\mathrm{~m}, 2 \mathrm{H}), 1.40$ (td, $1 \mathrm{H}, J=12.5$ and 9.5 $\mathrm{Hz}$ ), 1.33 (td, $1 \mathrm{H}, J=13.4$ and $4.0 \mathrm{~Hz}$ ), 1.19 (t, 3H, $J=7.2 \mathrm{~Hz}), 0.89$ (s, 3H), 0.88 (d, $3 \mathrm{H}, J=7.0 \mathrm{~Hz}$ ), 0.87 (s, 9H), 0.85 (d, 3H, $J=7.0 \mathrm{~Hz}), 0.02$ (s, 3H), $0.01(\mathrm{~s}, 3 \mathrm{H}) .{ }^{13} \mathbf{C} \mathbf{~ N M R}\left(\mathrm{CDCl}_{3}, 75.5 \mathrm{MHz}\right): \delta$ 210.2 (s), 172.9 (s), 136.7 (s), 125.6 (s), 101.4 (d), 76.2 (d), 69.6 (d), 63.8 (t), 51.0 (q), 49.3 (d), 46.1 (d), 42.0 (d), 40.4 (d), 39.1 (t), 37.2 (s), 34.6 (d), 33.5 (t), 31.6 (t), 29.6 (d), 29.4 (q), 25.9 (q), 23.5 (t), 19.4 (q), 18.1 (s), 16.1 (q), 15.4 (q), 15.1 (q), -4.1 (q), -4.8 (q). IR (neat, $\mathrm{cm}^{-1}$ ): 2950, 2935, 2855, 1752, 
1710. LRMS ( $\mathrm{m} / \mathrm{z}$, relative intensity): $548\left(\mathrm{M}^{+}, 20\right), 491$ (45), 417 (100), 74 (65). HRMS calcd for $\mathrm{C}_{31} \mathrm{H}_{52} \mathrm{O}_{6} \mathrm{Si}: 548.3533$, found: 548.3538. m.p. $\left({ }^{\circ} \mathrm{C}\right): 172-173$.

28b: ${ }^{1} \mathbf{H}$ NMR $\left(\mathrm{CDCl}_{3}, 600 \mathrm{MHz}\right): \delta 4.96(\mathrm{t}, 1 \mathrm{H}, J=7.1 \mathrm{~Hz}), 4.60$ (dm, $\left.1 \mathrm{H}, J=9.7 \mathrm{~Hz}\right), 3.79(\mathrm{dq}, 1 \mathrm{H}$, $J=9.7$ and $7.2 \mathrm{~Hz}$ ), $3.65(\mathrm{~s}, 3 \mathrm{H}), 3.47(\mathrm{dq}, 1 \mathrm{H}, J=9.7$ and $7.2 \mathrm{~Hz}), 3.12(\mathrm{td}, 1 \mathrm{H}, J=10.0 \mathrm{and} 4.6 \mathrm{~Hz})$, 2.98 (t, 1H, $J=9.7 \mathrm{~Hz}), 2.53-2.51(\mathrm{~m}, 1 \mathrm{H}), 2.35$ (dm, 1H, $J=18.3 \mathrm{~Hz}), 2.22-2.12$ (m, $2 \mathrm{H}), 2.16$ (s, 3H), 2.01-1.99 (m, 1H), 1.78-1.66 (m, 5H), 1.63-1.53 (m, 1H), 1.49-1.42 (m, 1H), 1.25-1.17 (m, 1H), $1.21(\mathrm{t}, 3 \mathrm{H}, J=7.2 \mathrm{~Hz}), 0.93-0.89(\mathrm{~m}, 9 \mathrm{H}), 0.88(\mathrm{~s}, 9 \mathrm{H}), 0.03(\mathrm{~s}, 3 \mathrm{H}), 0.02(\mathrm{~s}, 3 \mathrm{H}) .{ }^{13} \mathrm{C} \mathrm{NMR}\left(\mathrm{CDCl}_{3}\right.$, $75.5 \mathrm{MHz}$ ): $\delta 211.2$ (s), 175.1 (s), 135.6 (s), 131.1 (s), 98.1 (d), 76.5 (d), 63.3 (d), 63.0 (t), 51.4 (d), 47.9 (d), 47.6 (d), 41.2 (d), 37.3 (s), $35.1(\mathrm{~d}), 34.6(\mathrm{t}), 34.4(\mathrm{~d}), 30.9$ (t), 29.6 (q), 29.1 (q), 25.9 (q), 23.6 (t), 18.1 (s), $16.6(\mathrm{q}), 15.2$ (q), 14.9 (q), -4.1 (q), -4.7 (q). IR (neat, $\mathrm{cm}^{-1}$ ): 2950, 2931, 2889, 2855, 1737, 1706. LRMS (m/z, relative intensity): 548 ( $\mathrm{M}^{+}$, 5), 502 (90), 445 (80), 417 (80), 327 (45), 74 (100). HRMS calcd for $\mathrm{C}_{31} \mathrm{H}_{52} \mathrm{O}_{6} \mathrm{Si}$ : 548.3533, found: 548.3538. m.p. $\left({ }^{\circ} \mathrm{C}\right)$ : 154-155.

[1] Love, B. E.; Jones, E. G. J. Org. Chem. 1999, 64, 3755-3756.

[2] Porter, N. A.; Scott, D. M.; Rosenstein, I. J.; Giese, B.; Veit, A.; Zeitz, H. G. J. Am. Chem. Soc. 1991, 113, 1791-1799.

[3] B. M. Trost, W. J. Frazee, J. Am. Chem. Soc. 1977, 99, 6124-6126.

[4] Bombala, M. U.; Ley, S. V. J. Chem. Soc., Perkin Trans. I 1979, 3013-3016.

[5] Hassfeld, J.; Christmann, M.; Kalesse, M. Org. Lett. 2001, 3, 3561-3564. 


\section{Copies of ${ }^{1} \mathrm{H}$ NMR}

3-(tert-Butyldimethylsilyloxy)-1-propyne (9)

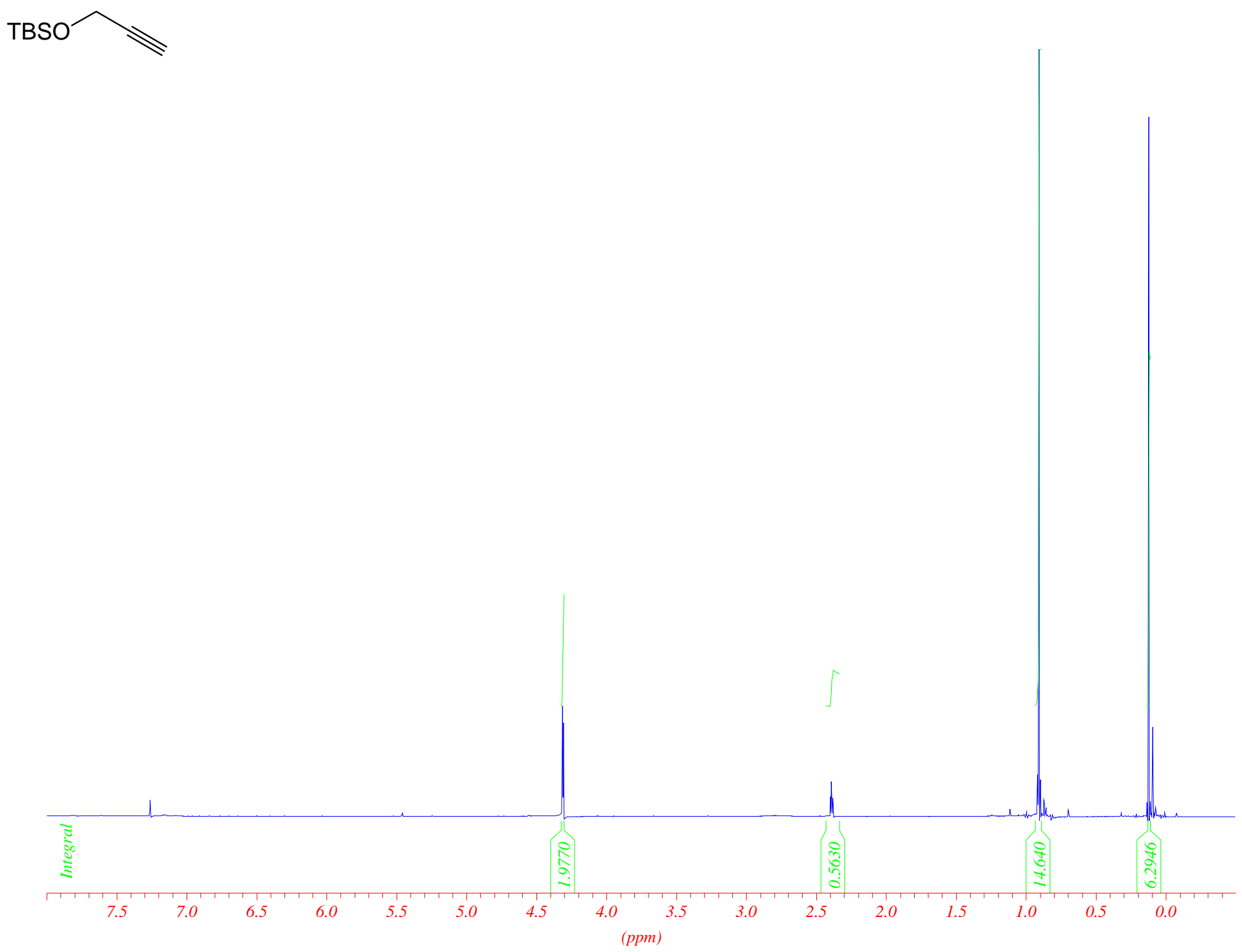


(5E)-1-(tert-Butyldimethylsilyloxy)-5-hepten-2-yn-4-ol (29)
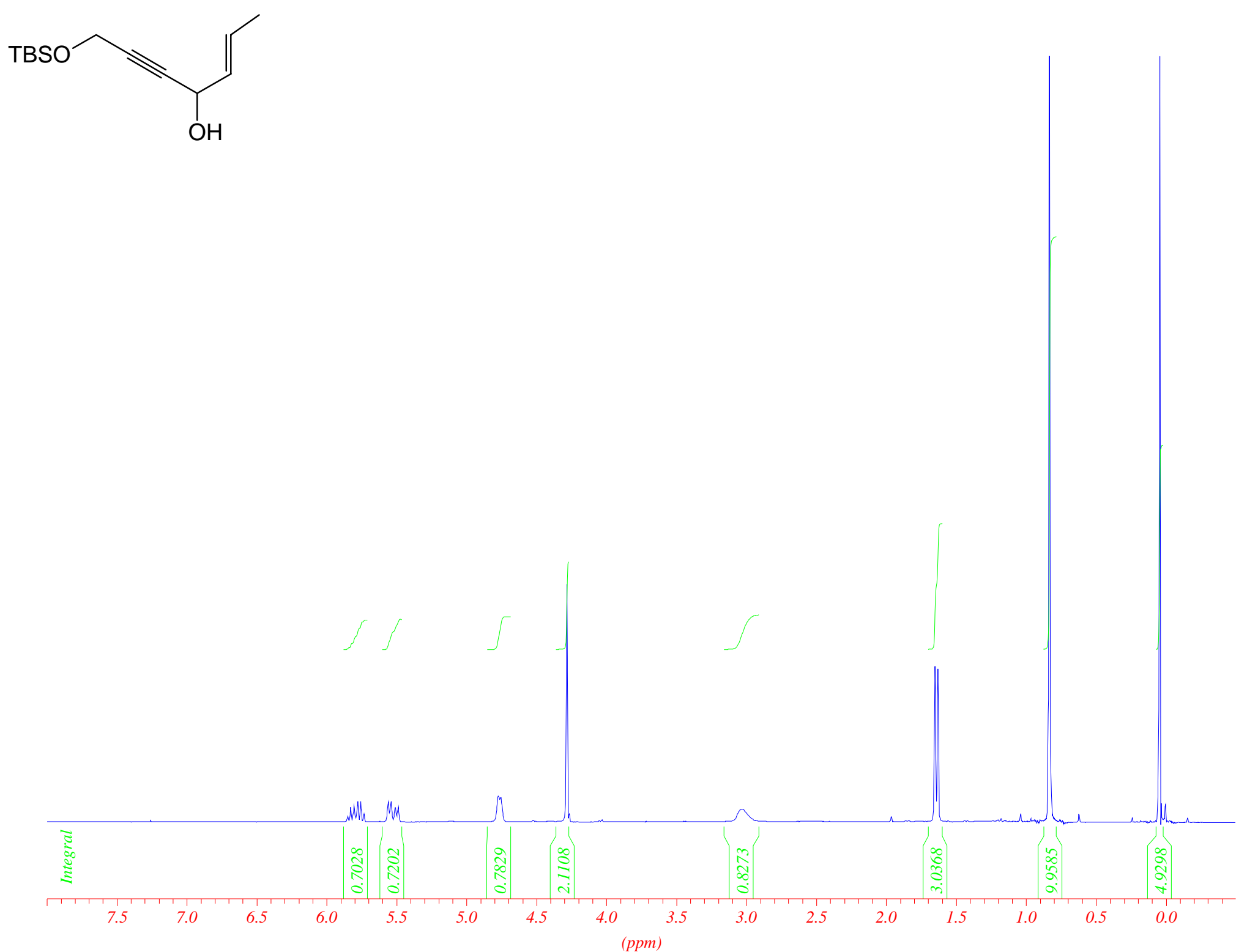
(5E)-1-(tert-Butyldimethylsilyloxy)-5-hepten-2-yn-4-one (10)
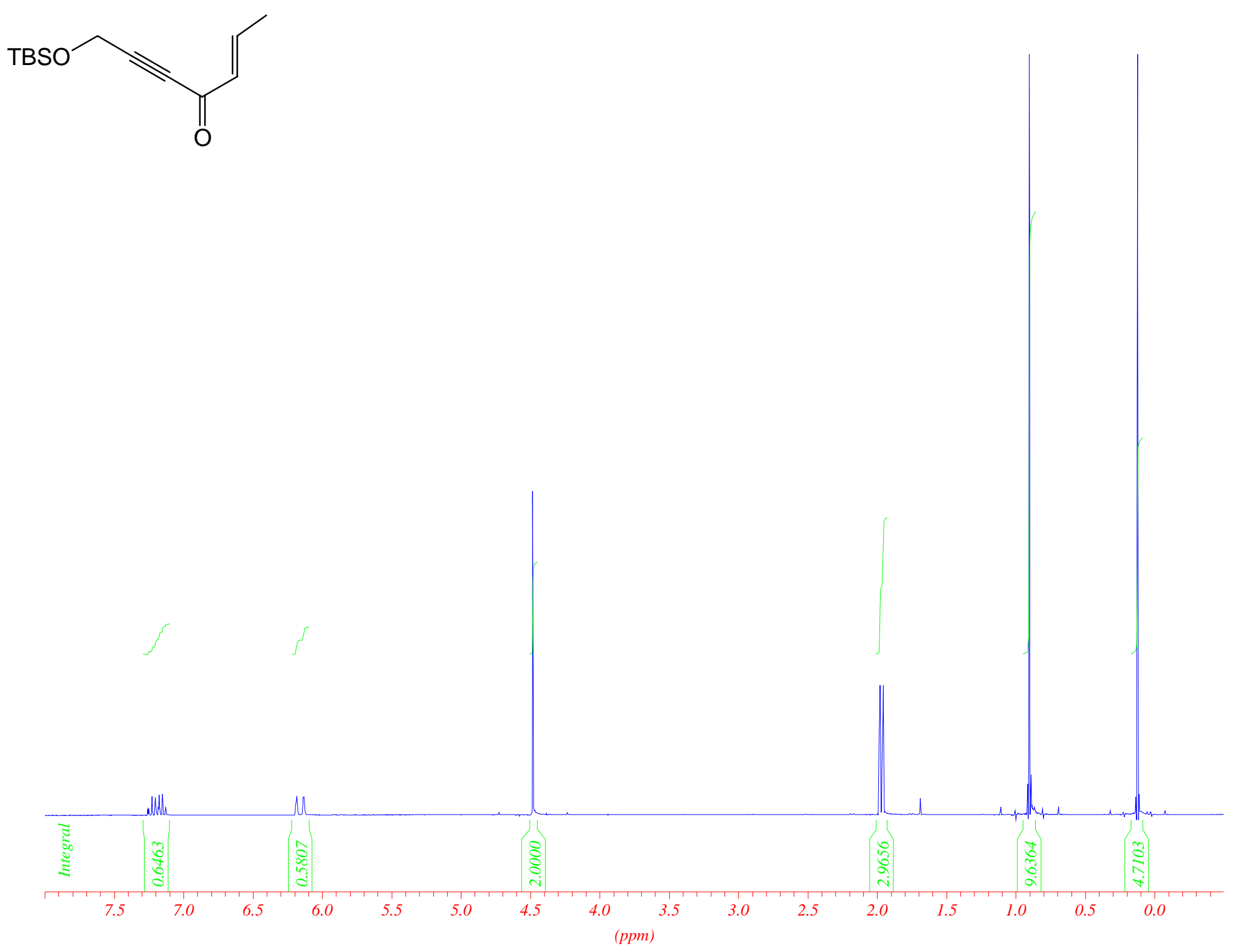
( \pm )-(5E)-1-(tert-butyldimethylsilyloxy)-4-(1,3-dithian-2-yl)-5-hepten-2-yn-4-ol (30)

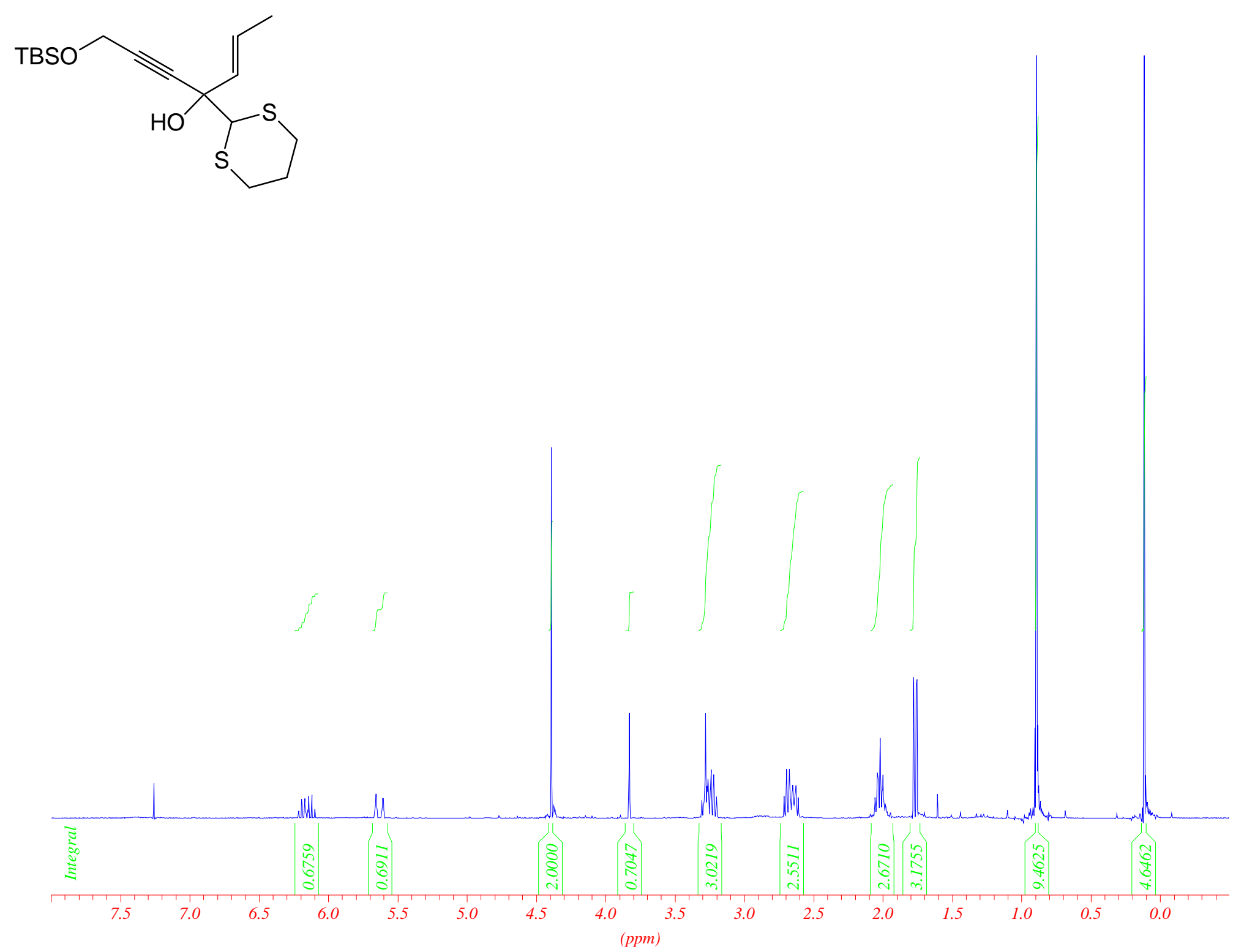


(士)-(5E)-4-Acetoxy-1-(tert-butyldimethylsilyloxy)-4-(1,3-dithian-2-yl)-5-hepten-2-yne (11)
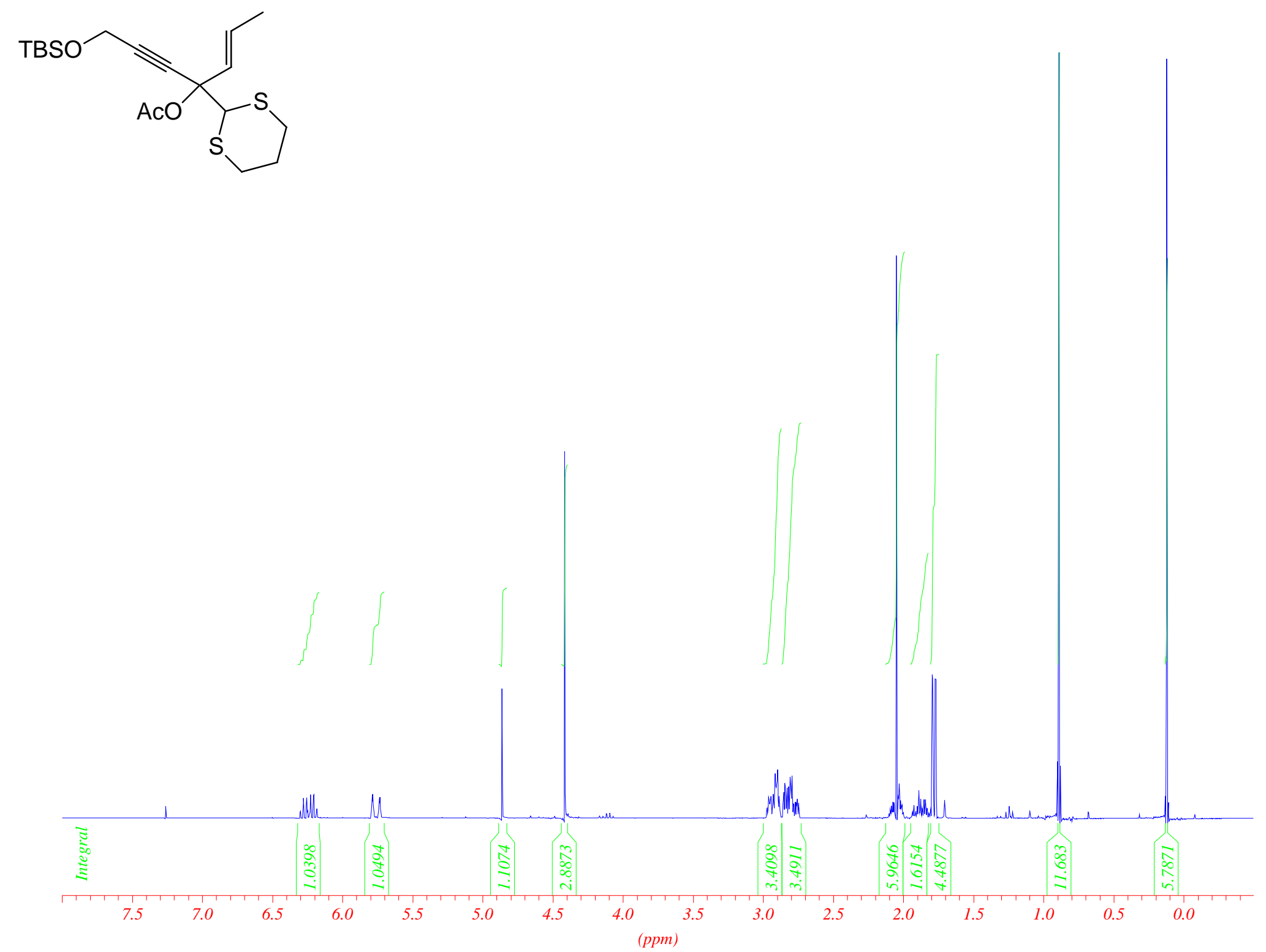
( \pm )-(5E)-4-(1,3-Dithian-2-yl)-2-methyl-2,3,5-heptatrien-1-ol (12)
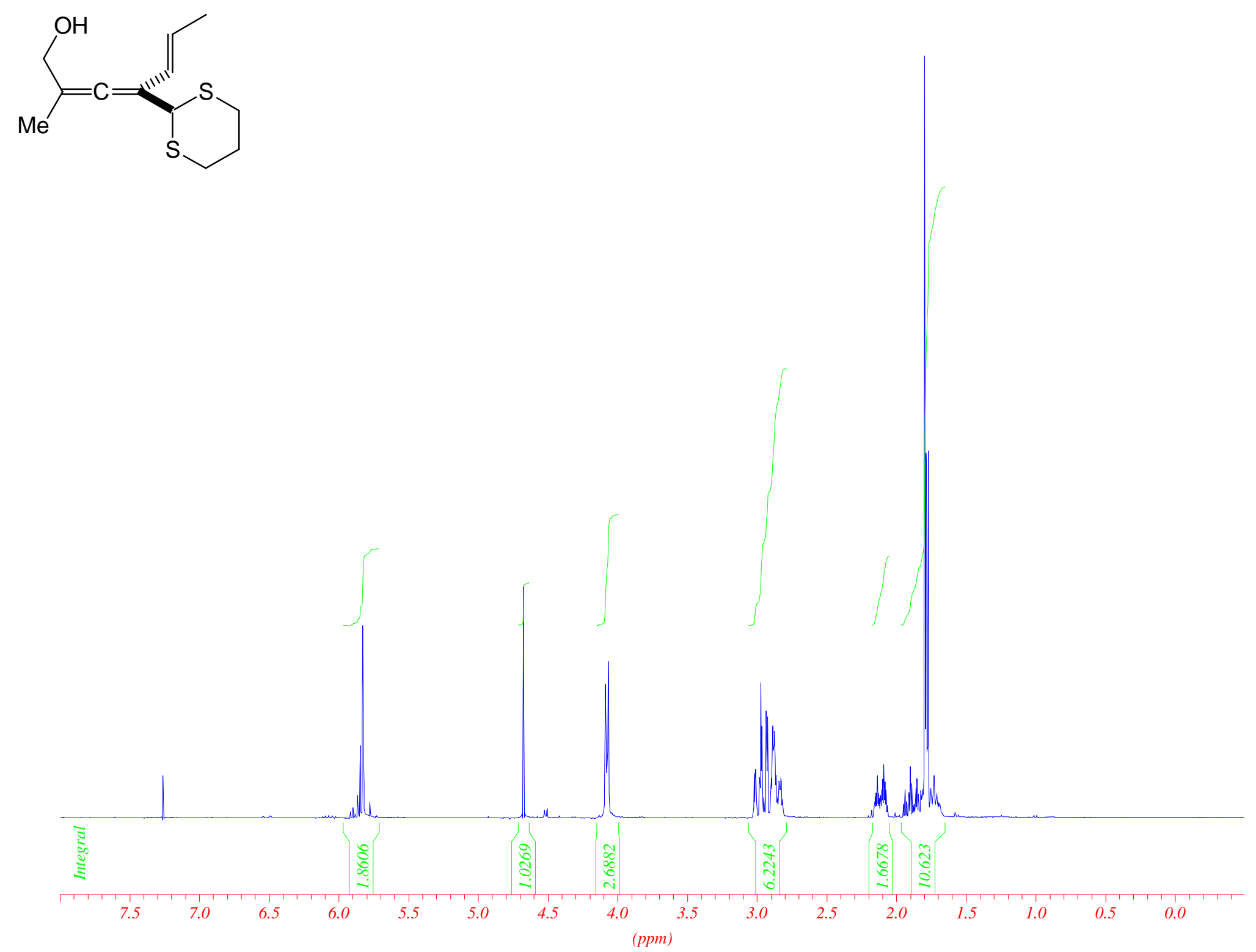


\section{Lactone 14a}
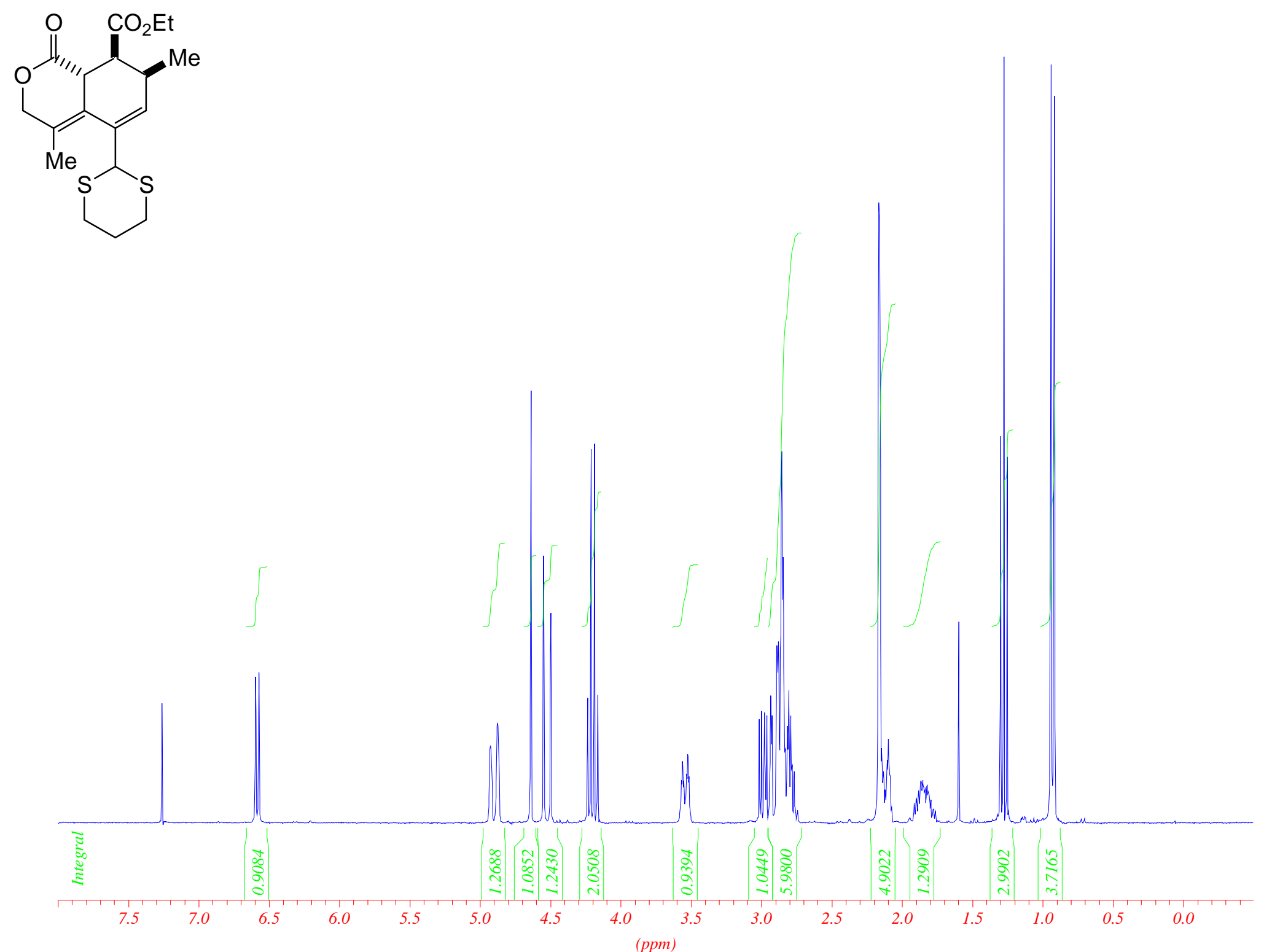


\section{Lactone 14b}

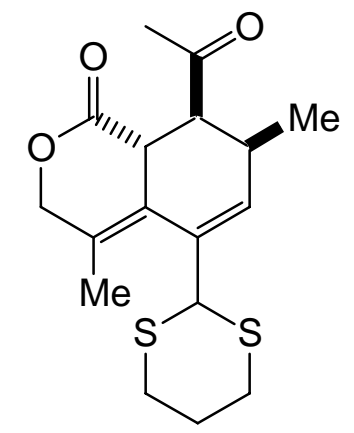


$\alpha, \beta$-Unsaturated aldehyde $15 a$
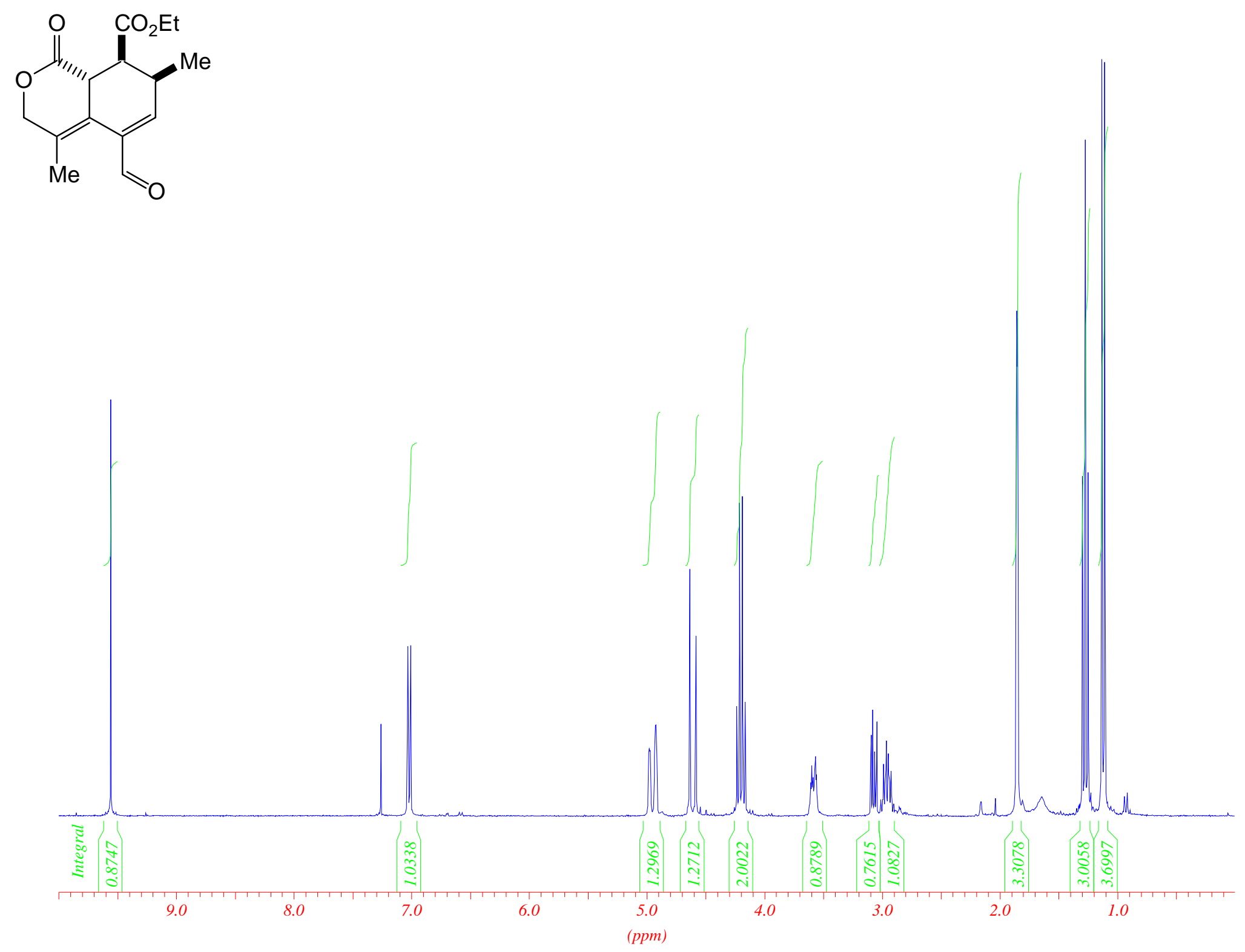
$\alpha, \beta$-Unsaturated aldehyde $15 b$

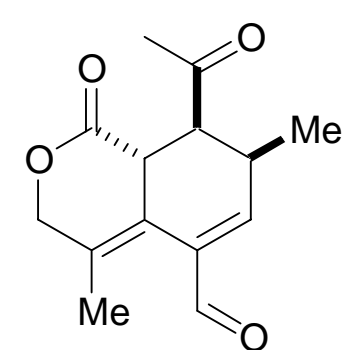




\section{Lactone 6a}

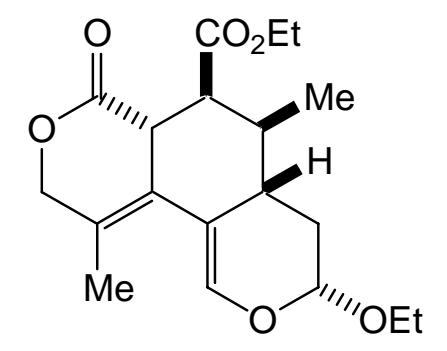




\section{Lactone 6b}

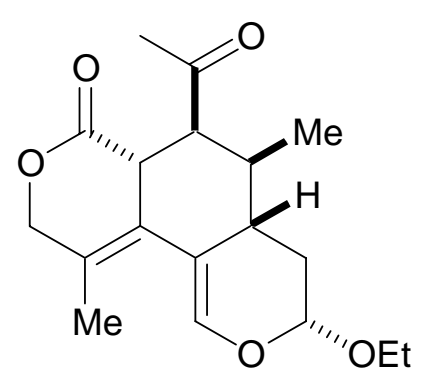




\section{Triene 7a}
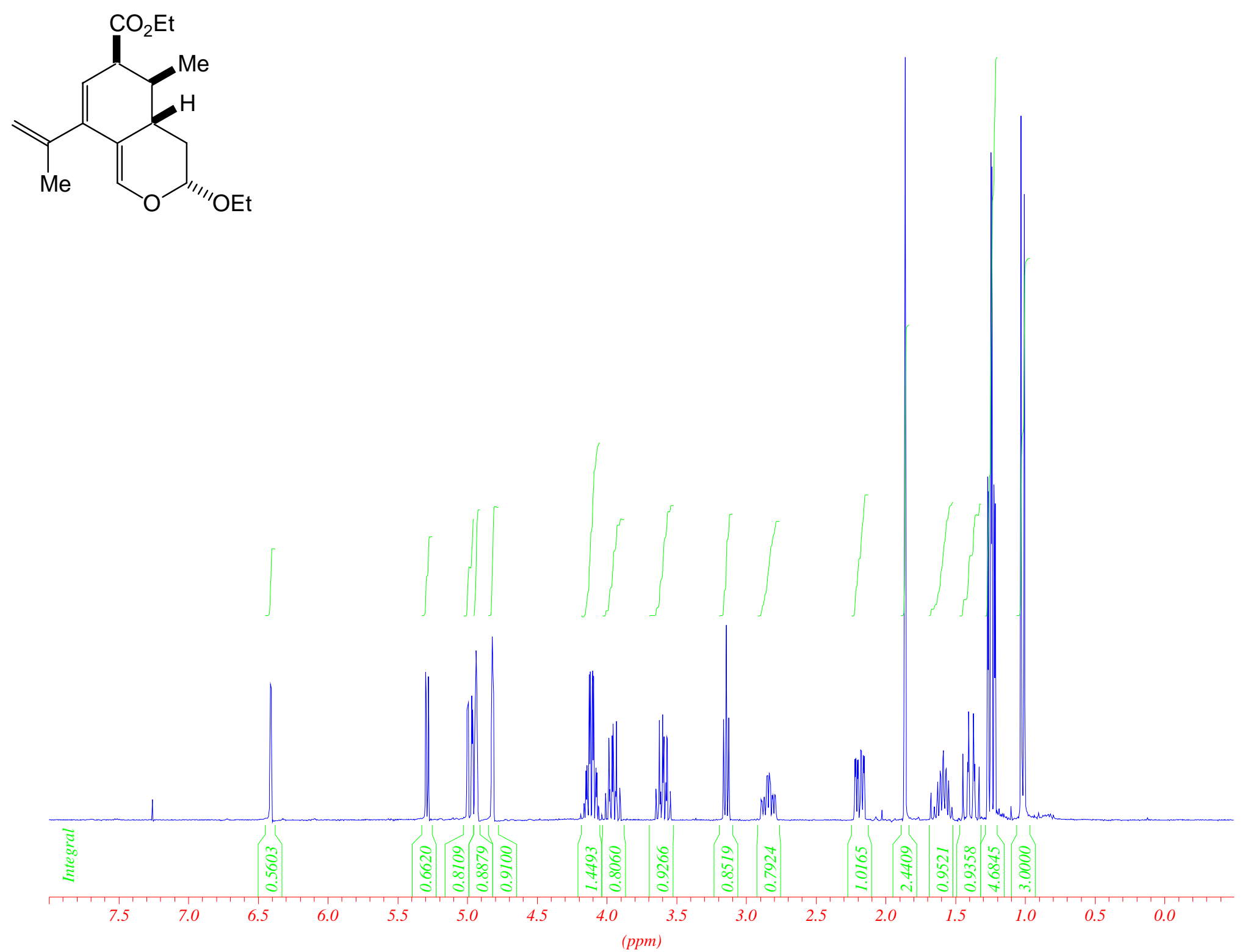
Triene 7b

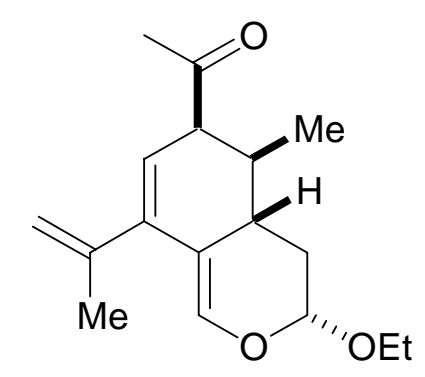




\section{Triester 19a}

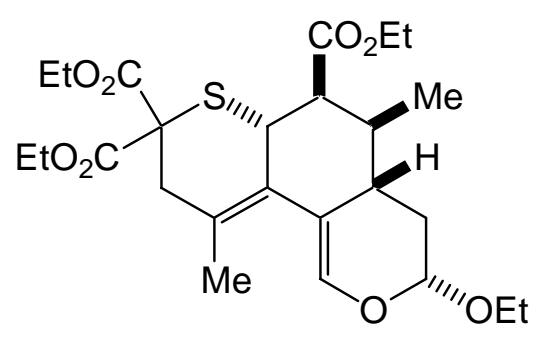




\section{Triester 20a}
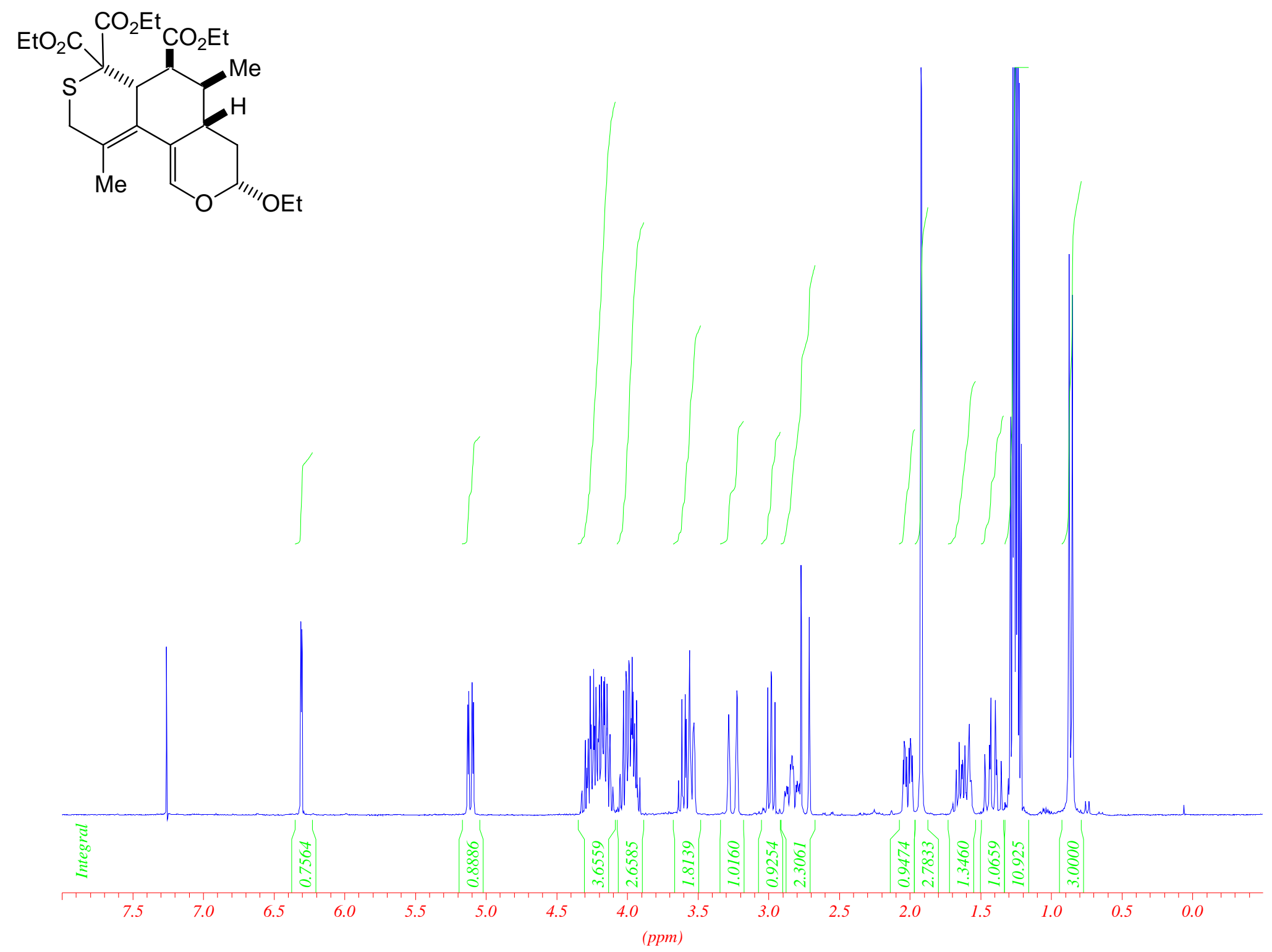


\section{Triester 19b}
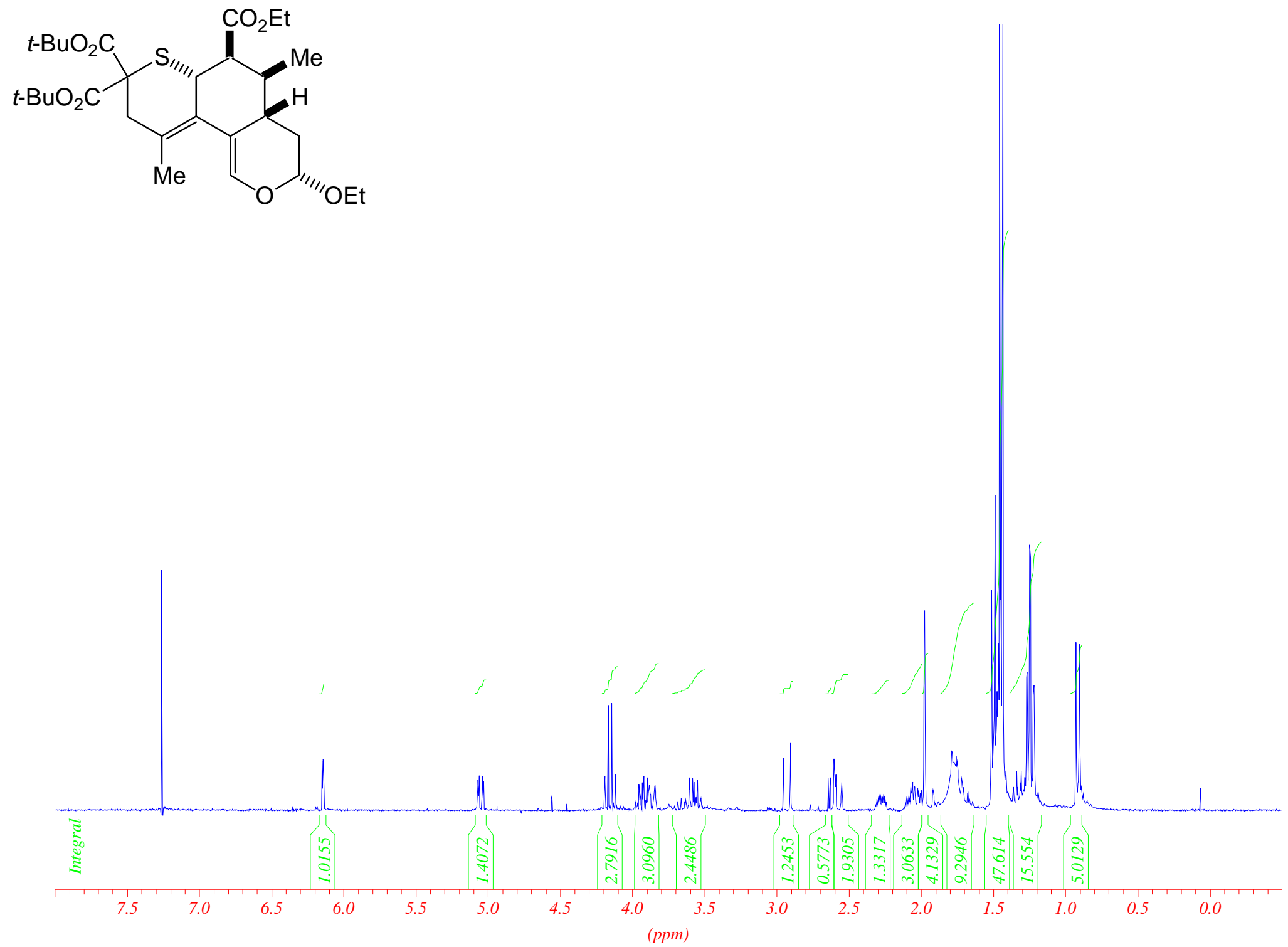


\section{Triester 20b}

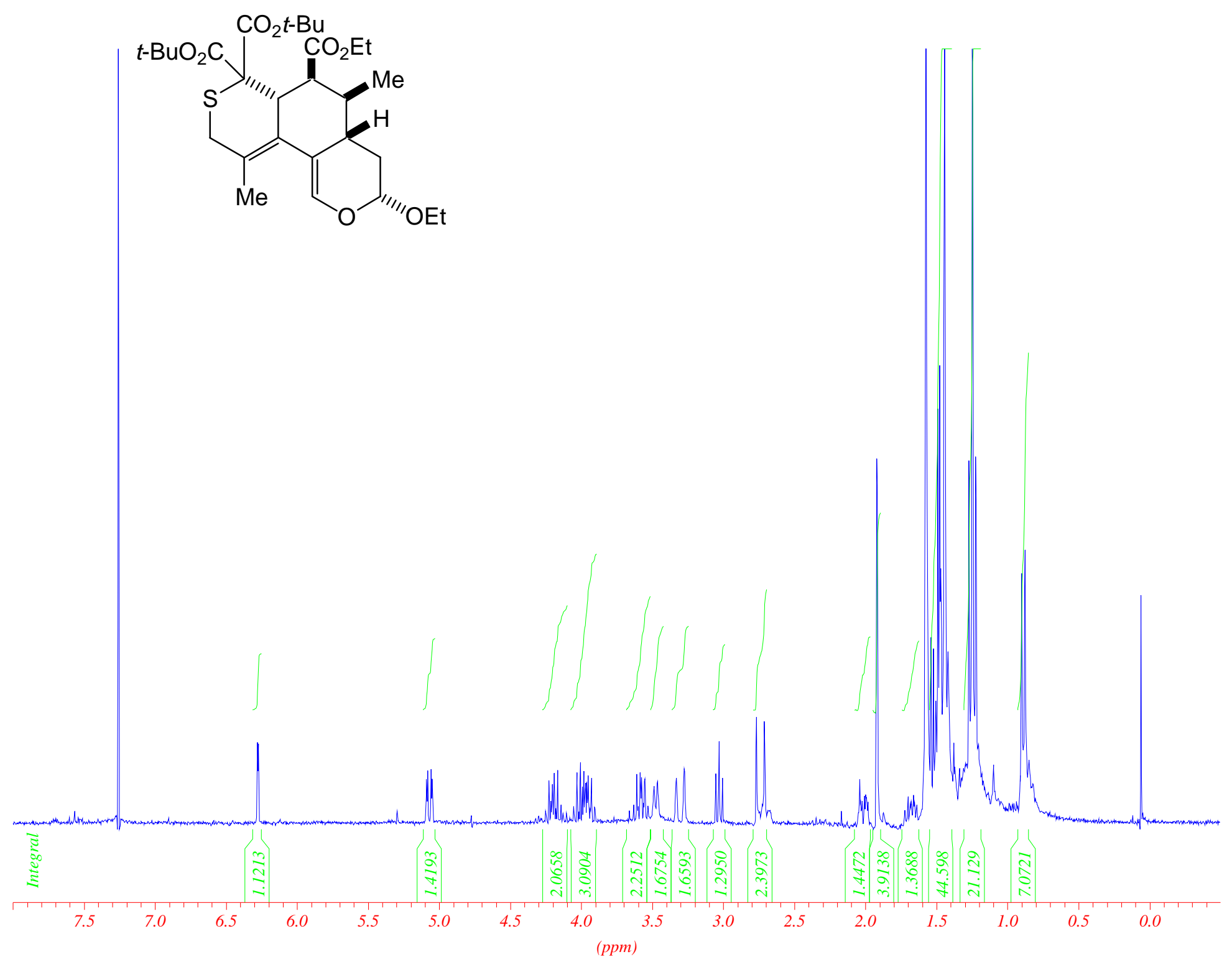


5-bromo-2,2-dimethyl-[1,3]dioxane-4,6-dione (33) used to make thione 18.

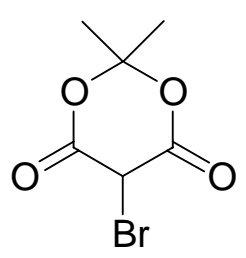

$\mathrm{Br}$

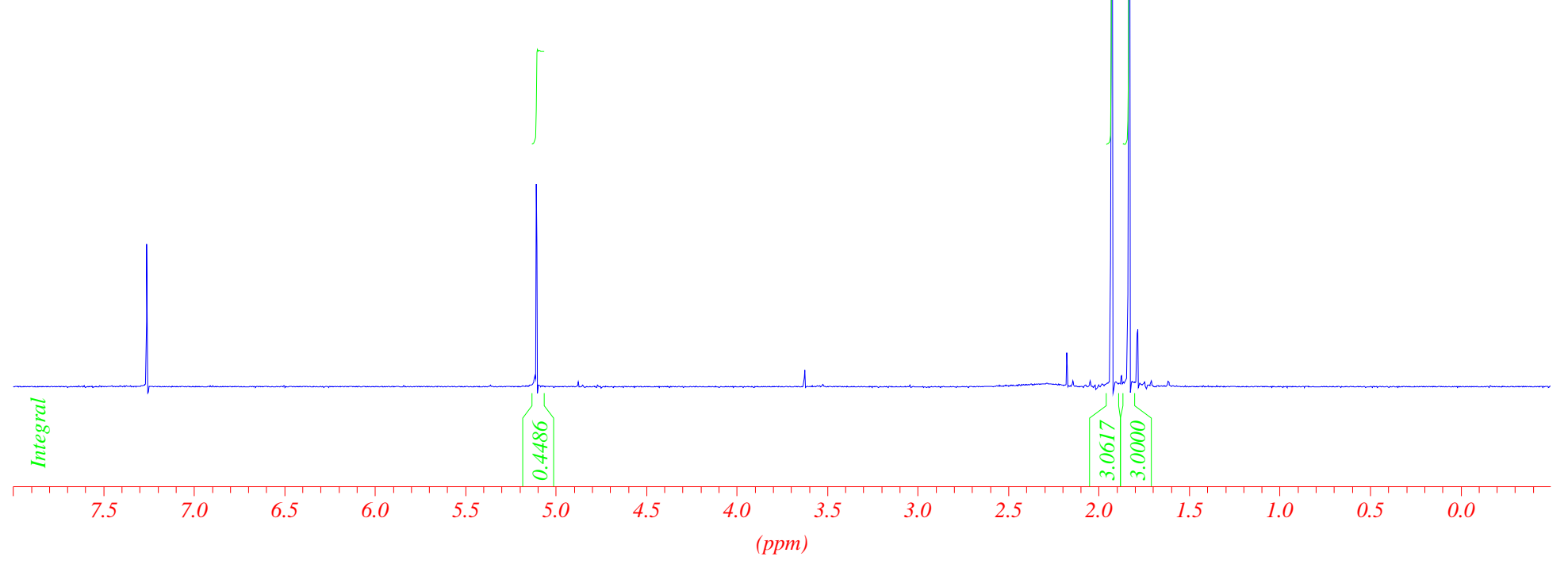




\section{Dioxane-4,6-dione 19c}
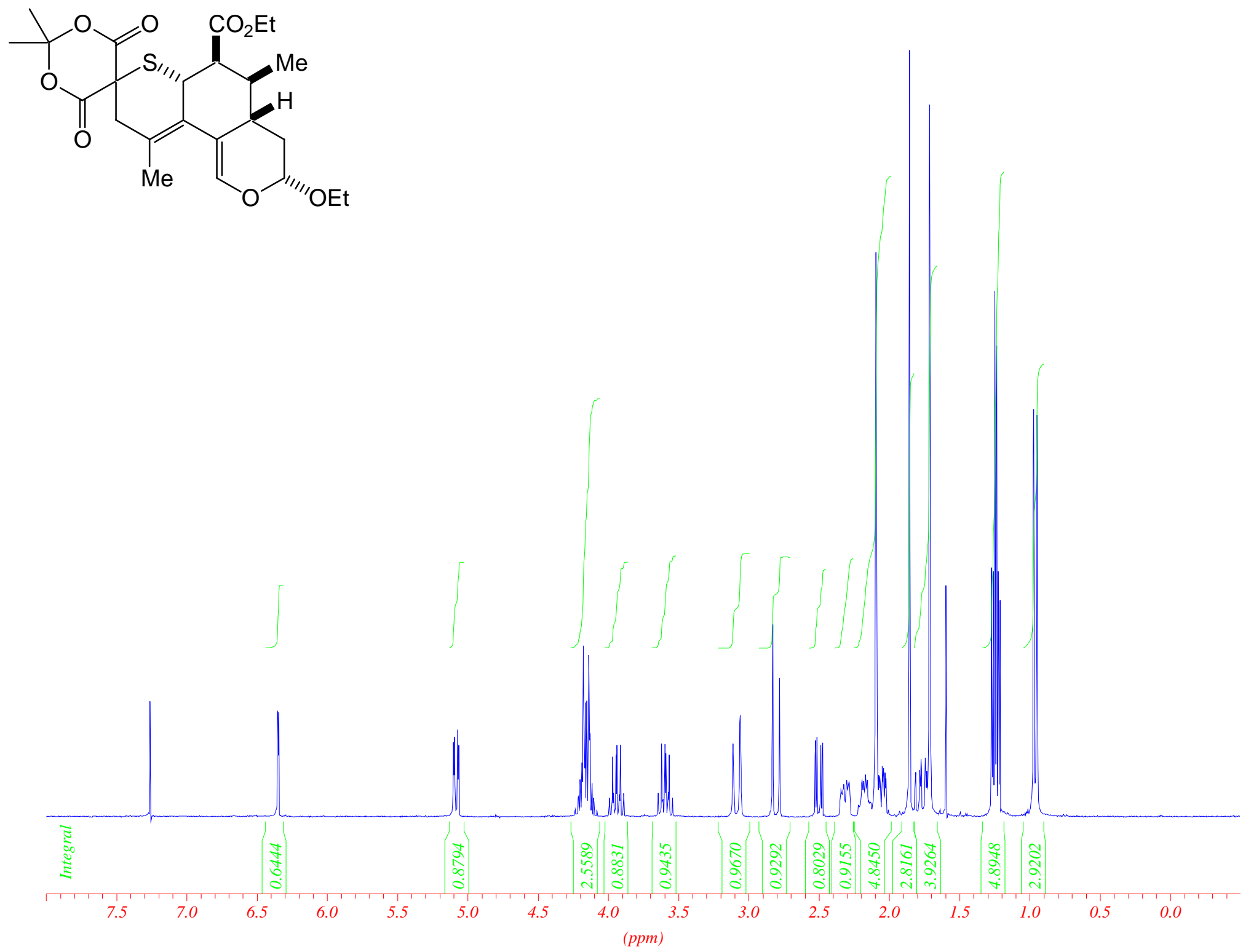


\section{Dioxane-4,6-dione 20c}
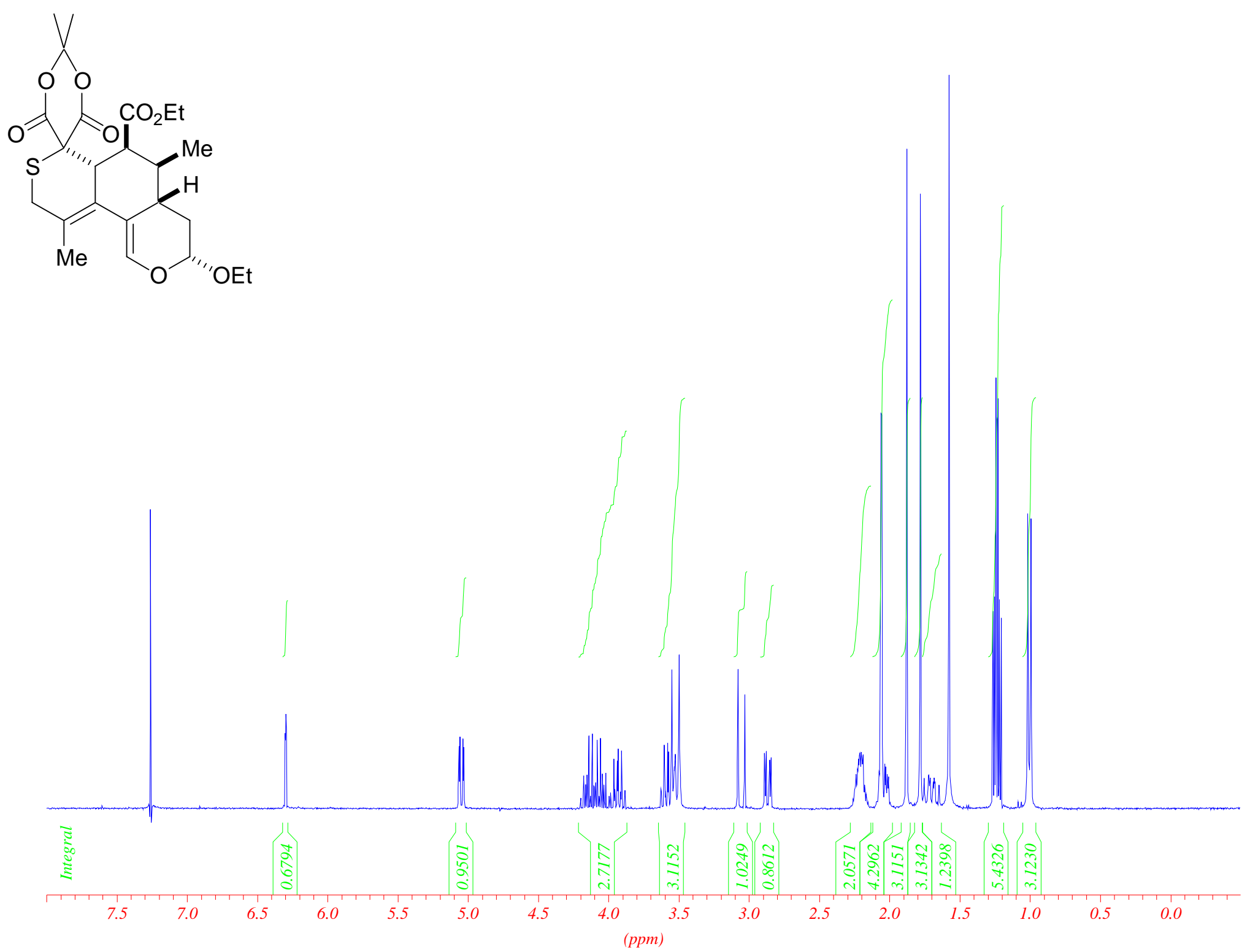
5-(phtalimide- $N$-sulfenyl)-2,2-dimethyl-[1,3]dioxane-4,6-dione (34) used to make thione 18.
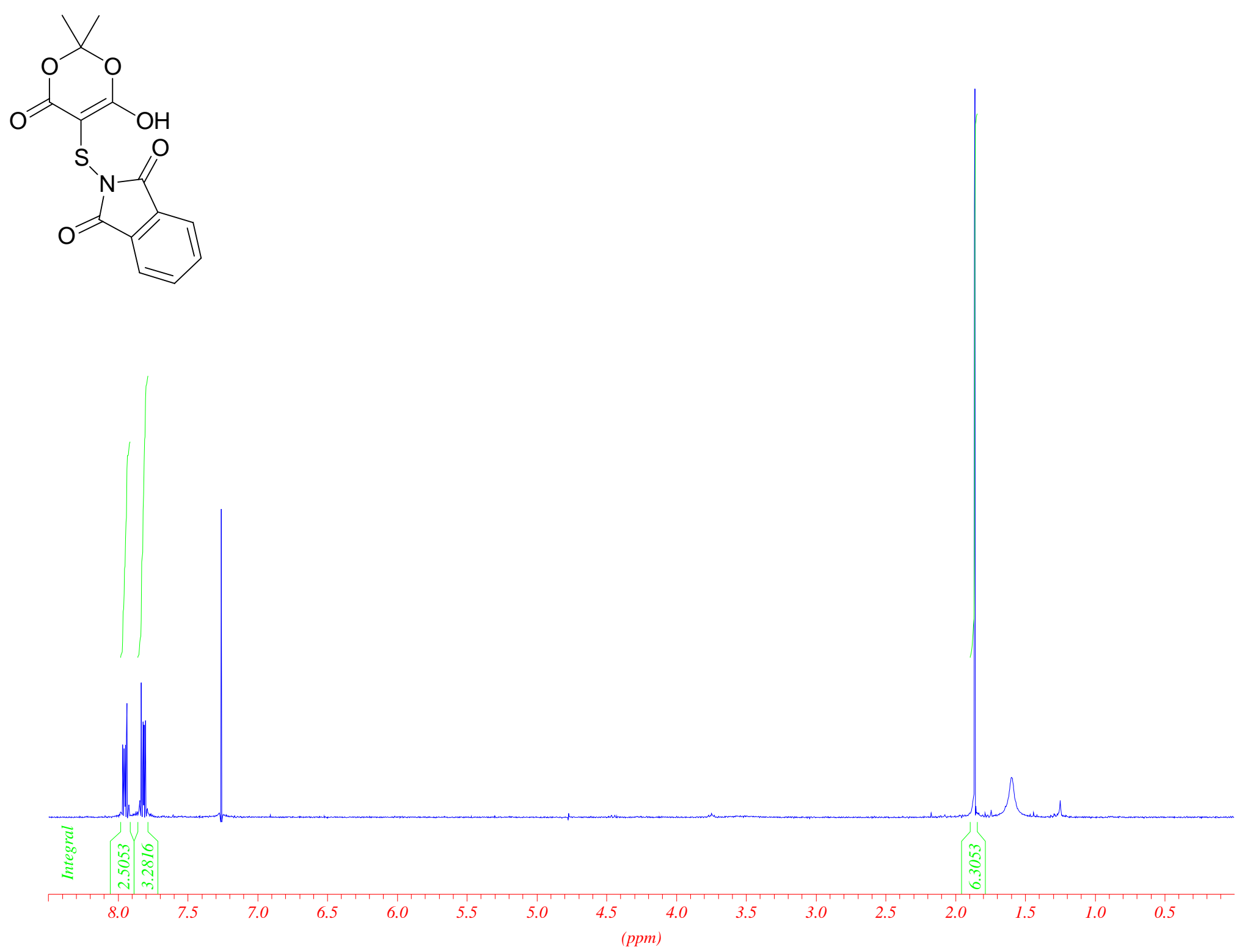
Dioxane-4,6-dione 21
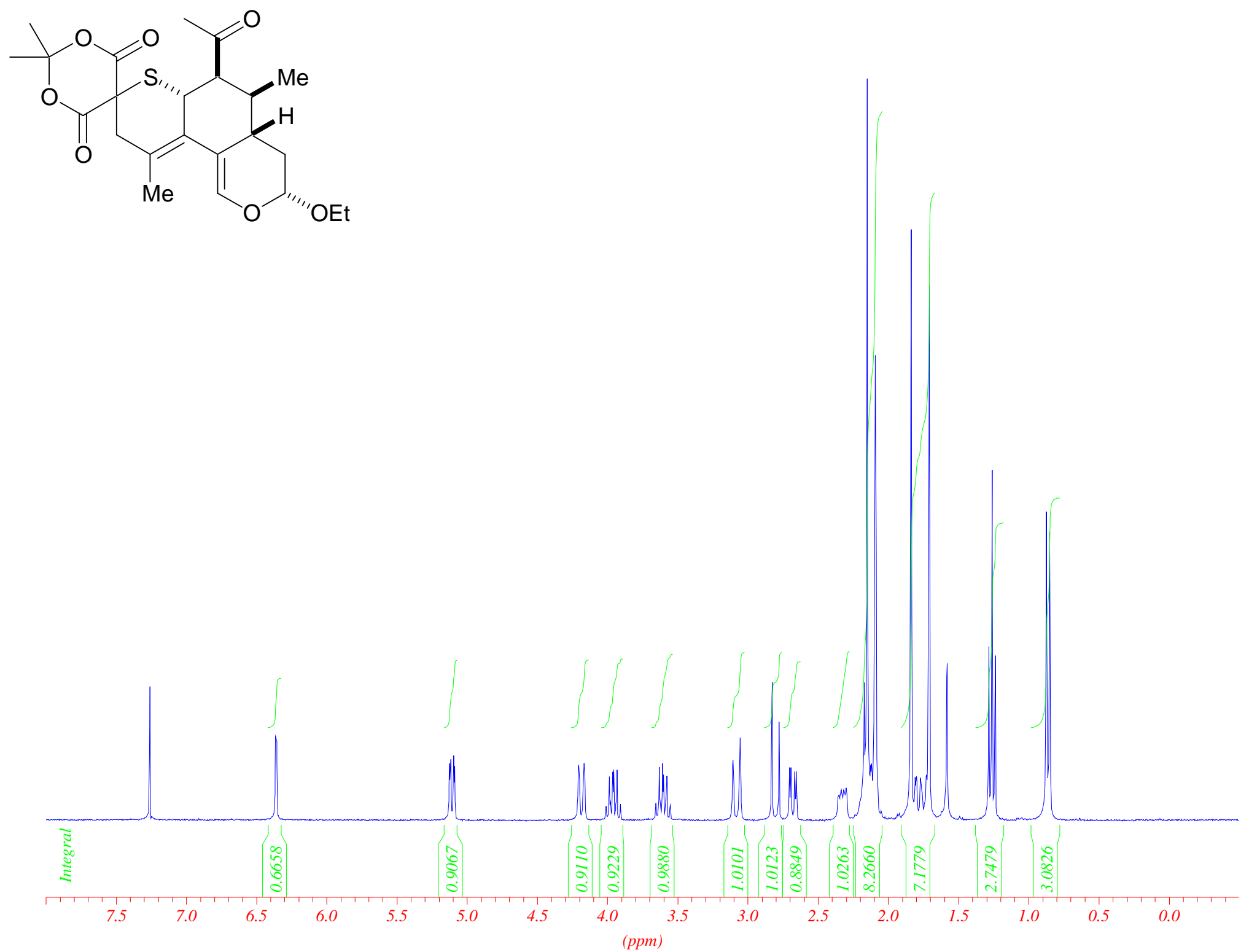
Dioxane-4,6-dione 22
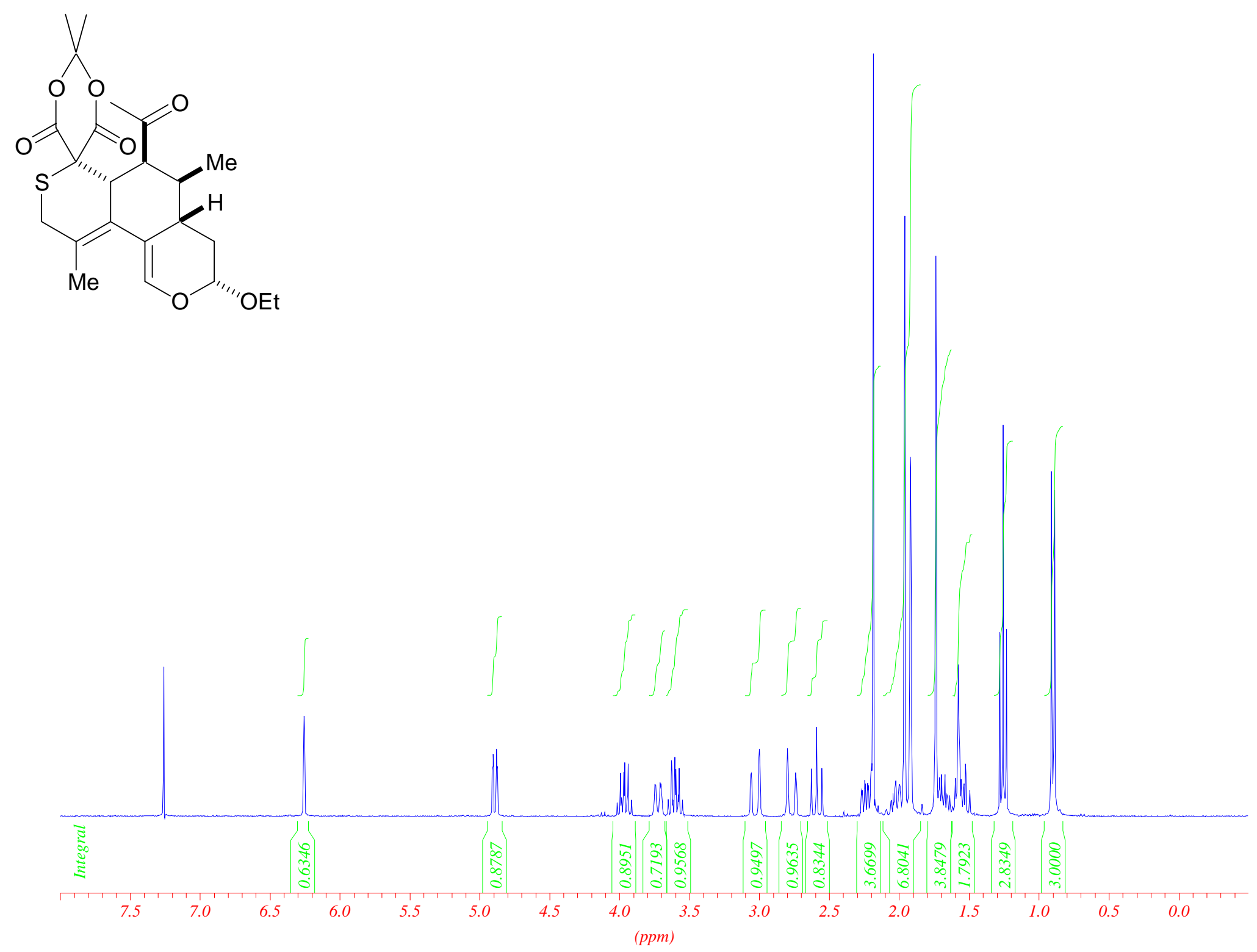


\section{Ester 35 (minor diastrereomer)}
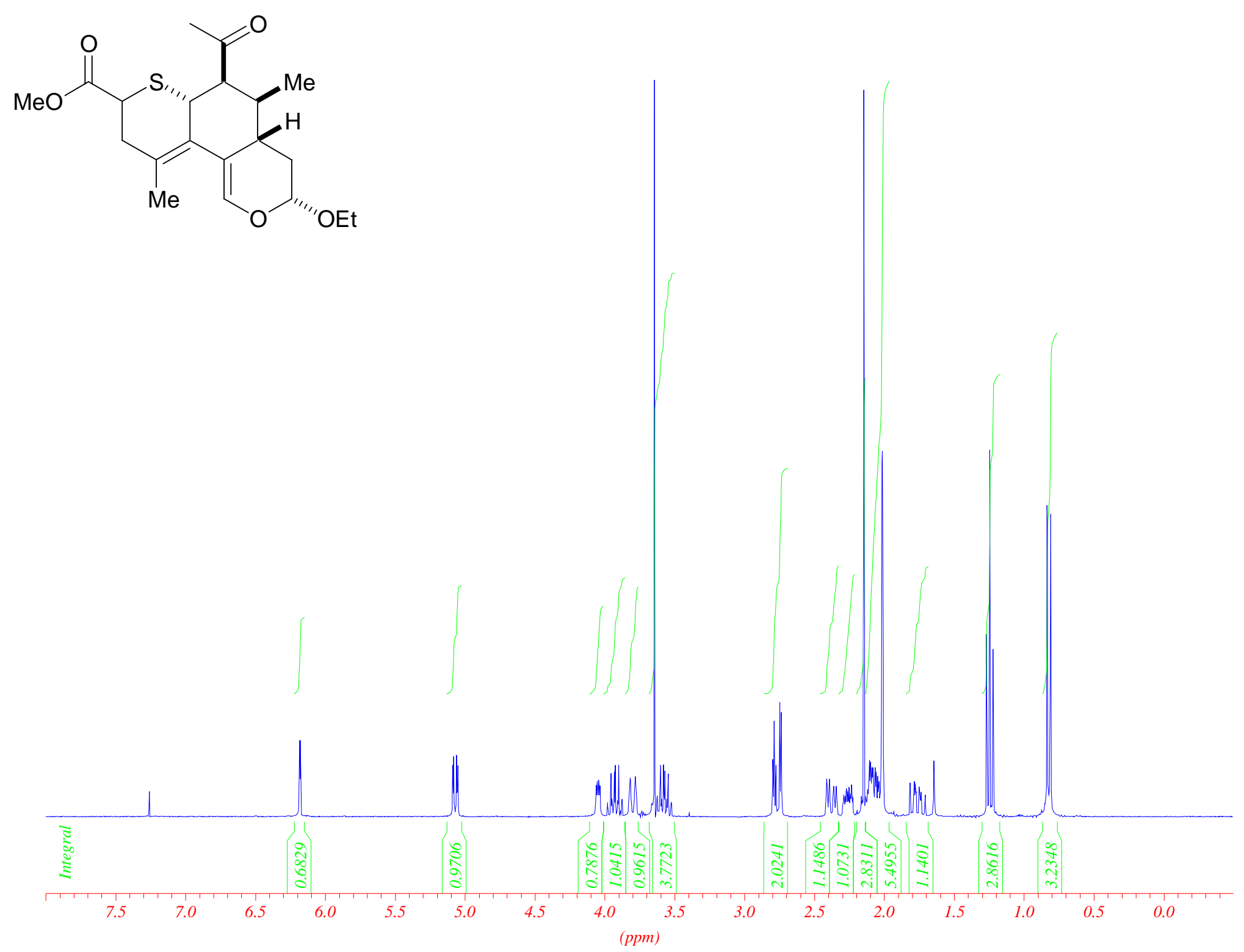


\section{Ester 35 (major diastrereomer)}
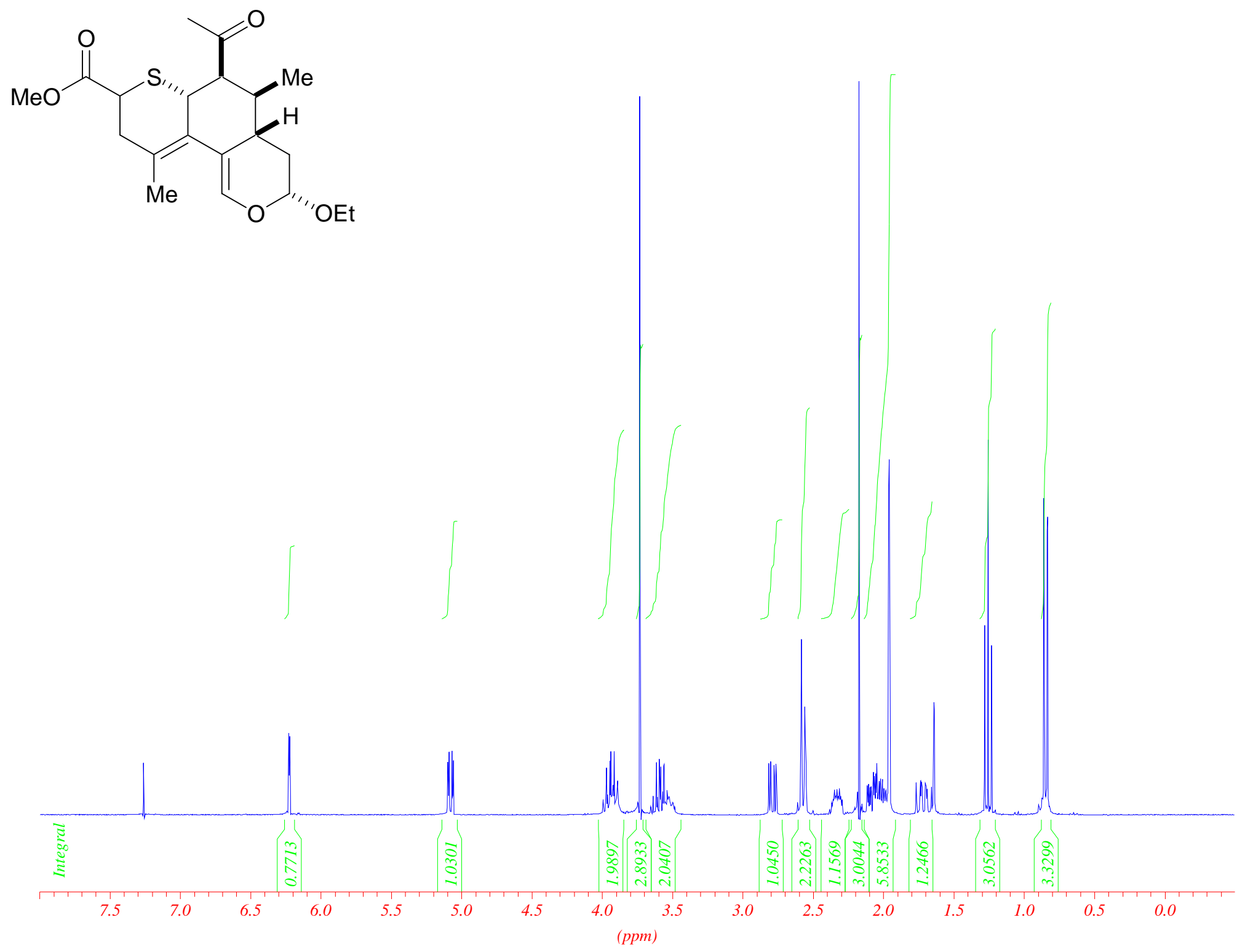
Mixture of diols 36
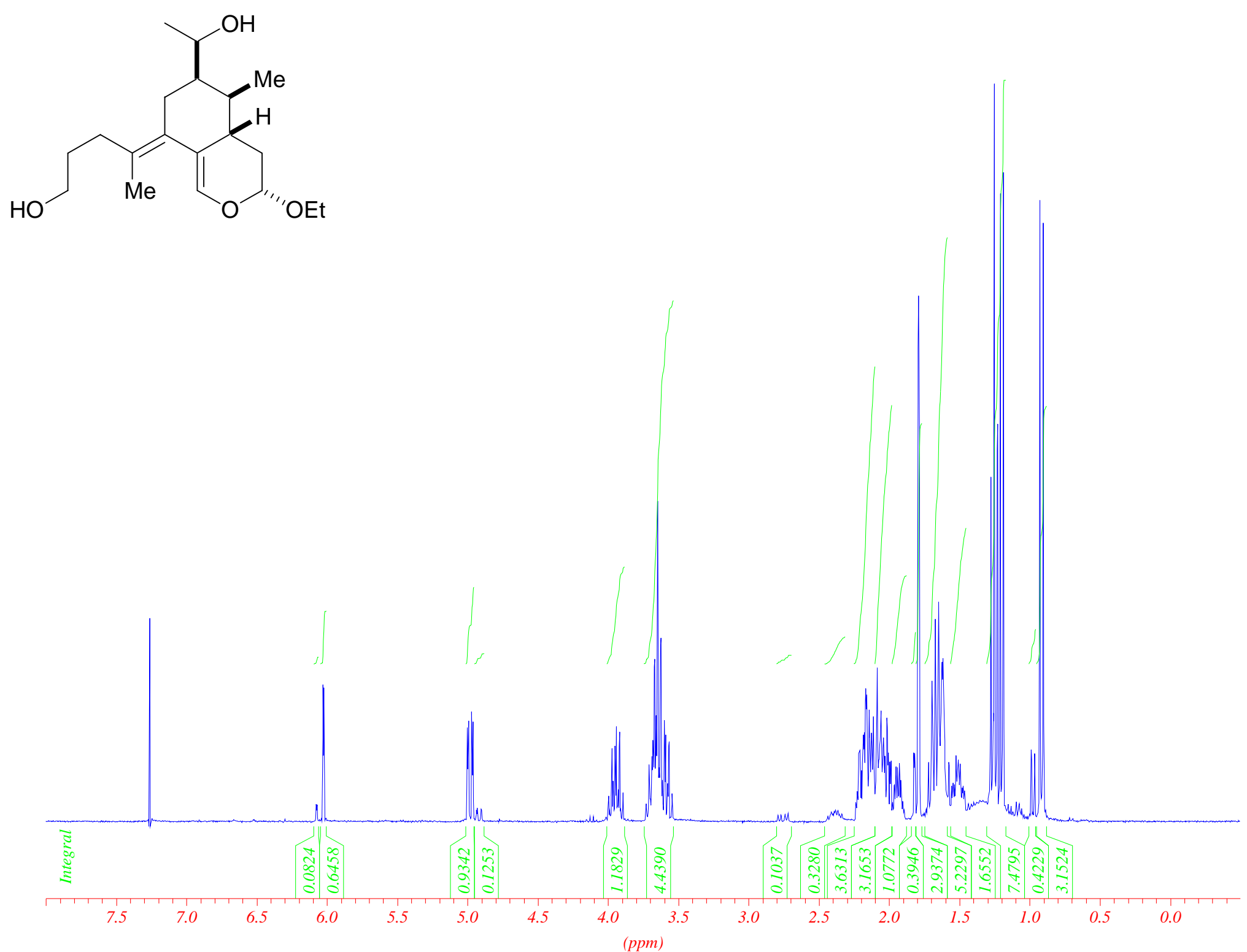
Aldehyde 24
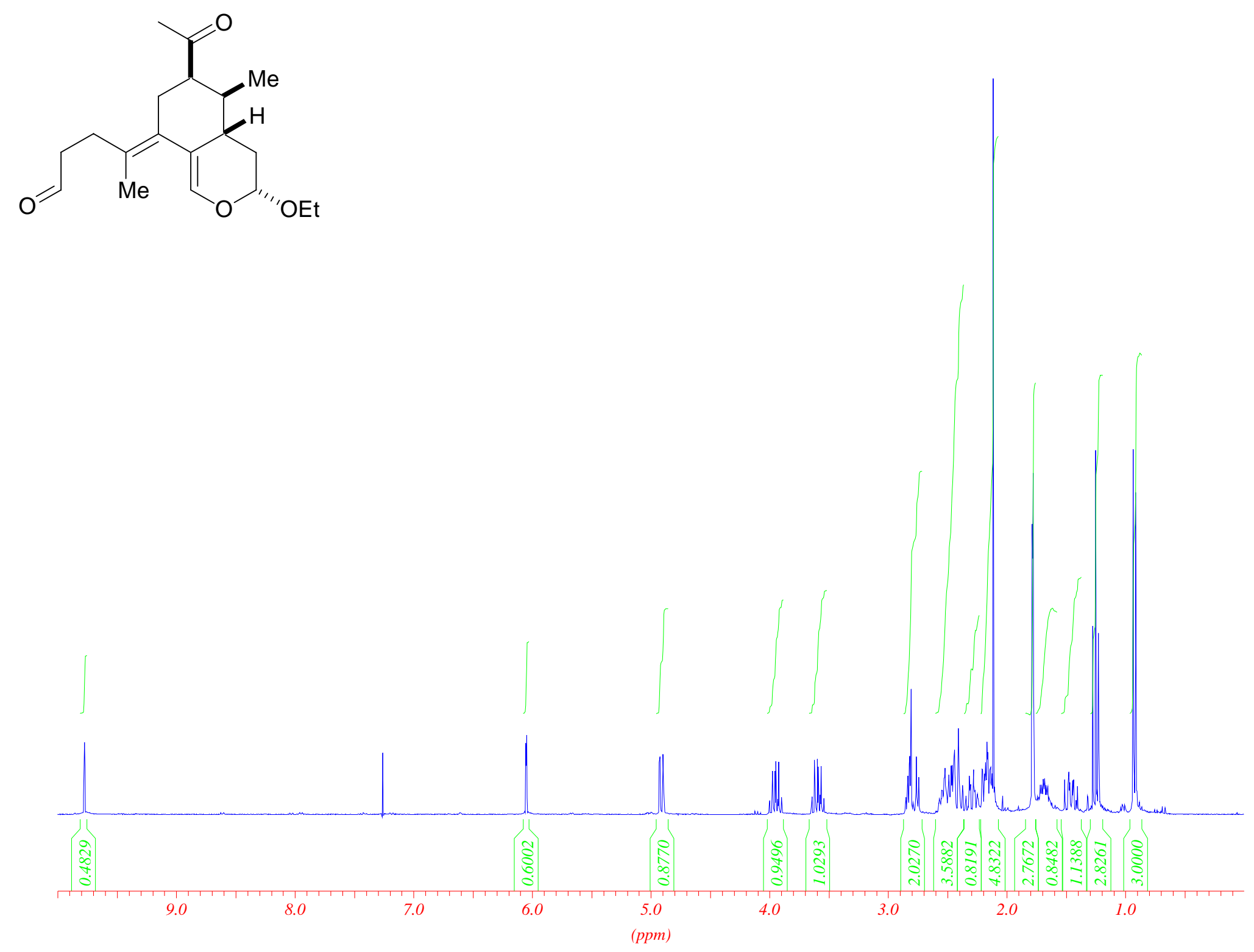
$\alpha, \beta$-Unsaturated esters $26 a$ and $26 b$
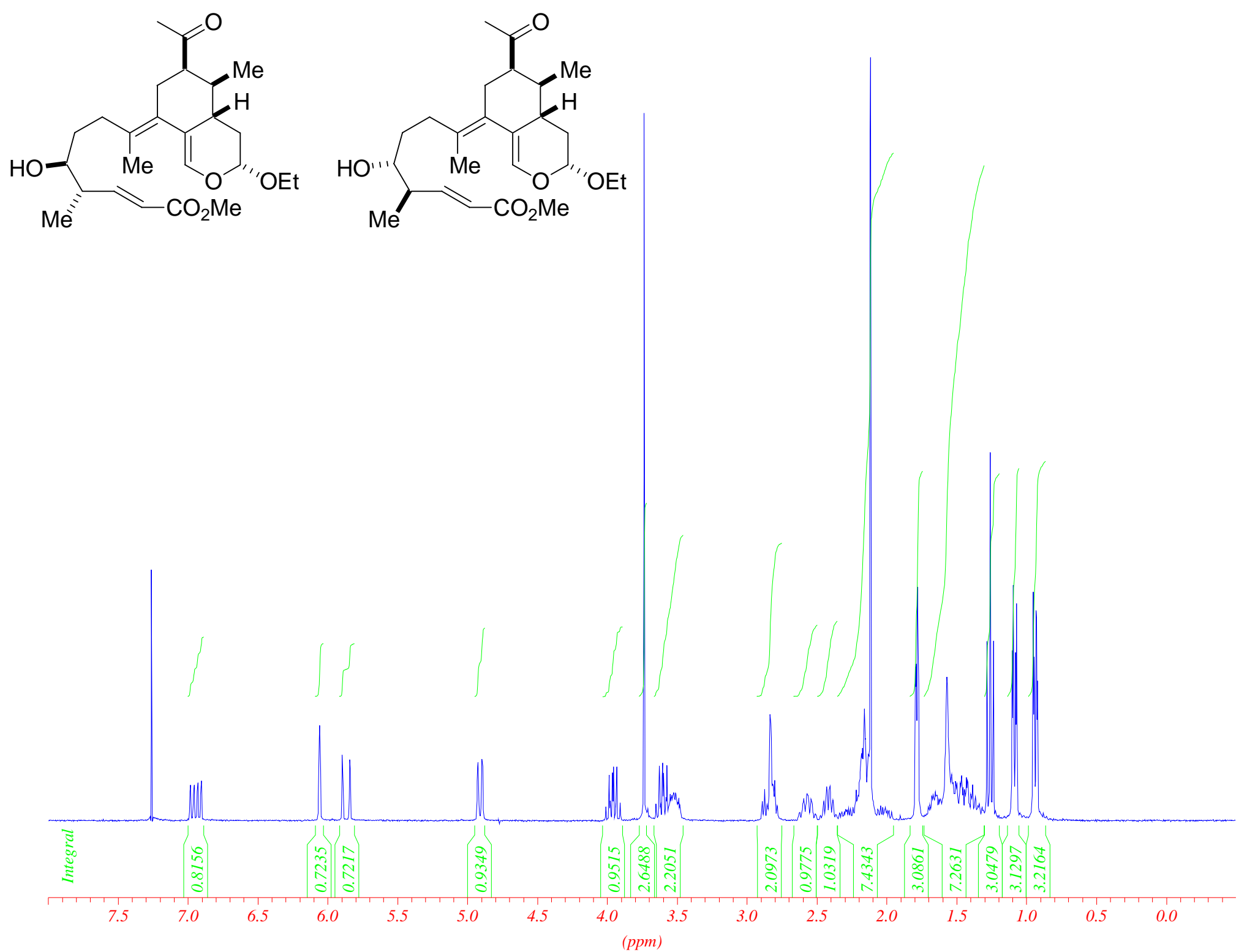
$\alpha, \beta$-Unsaturated esters $27 \mathrm{a}$ and $27 \mathrm{~b}$
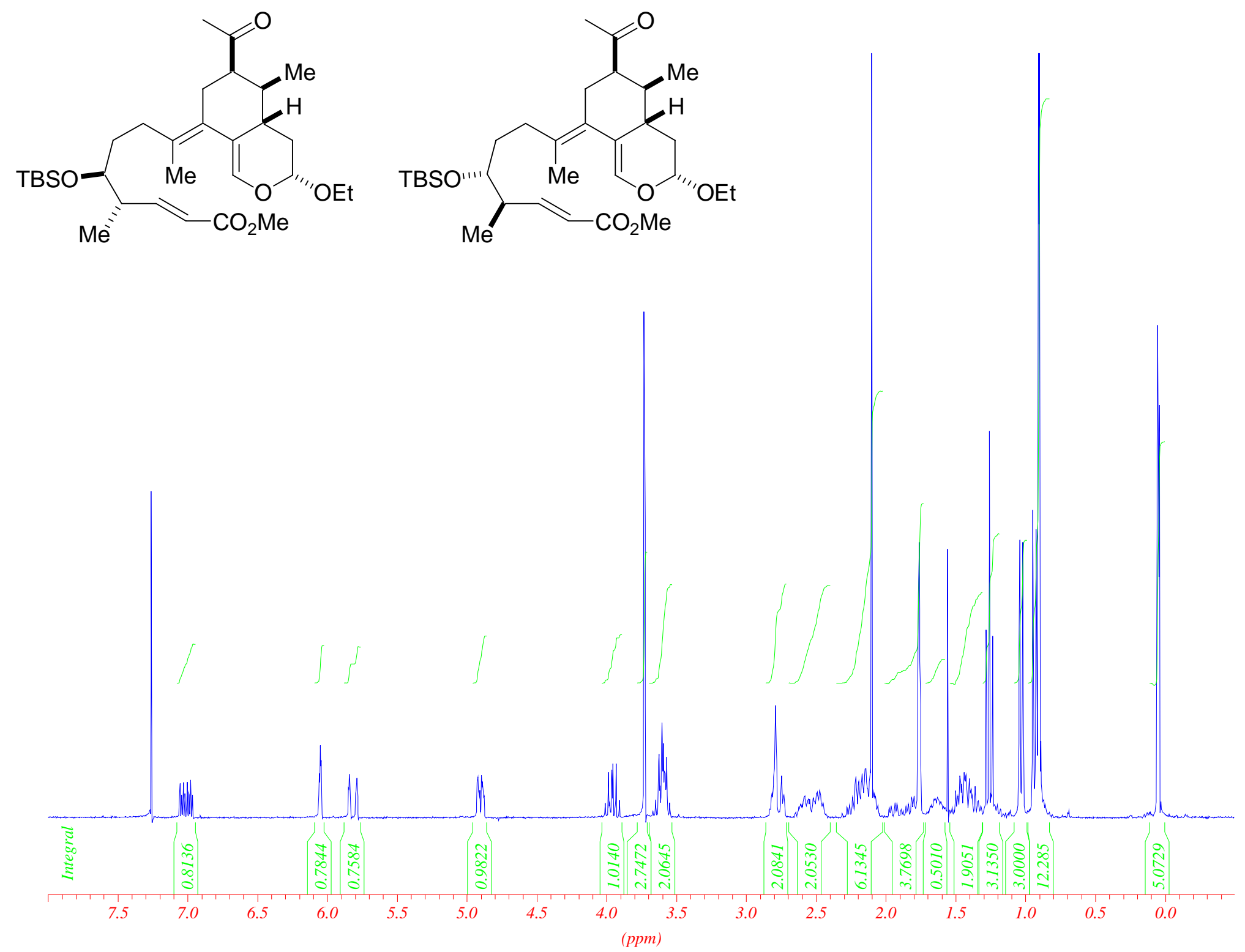


\section{Tetracyclic compound 28a}
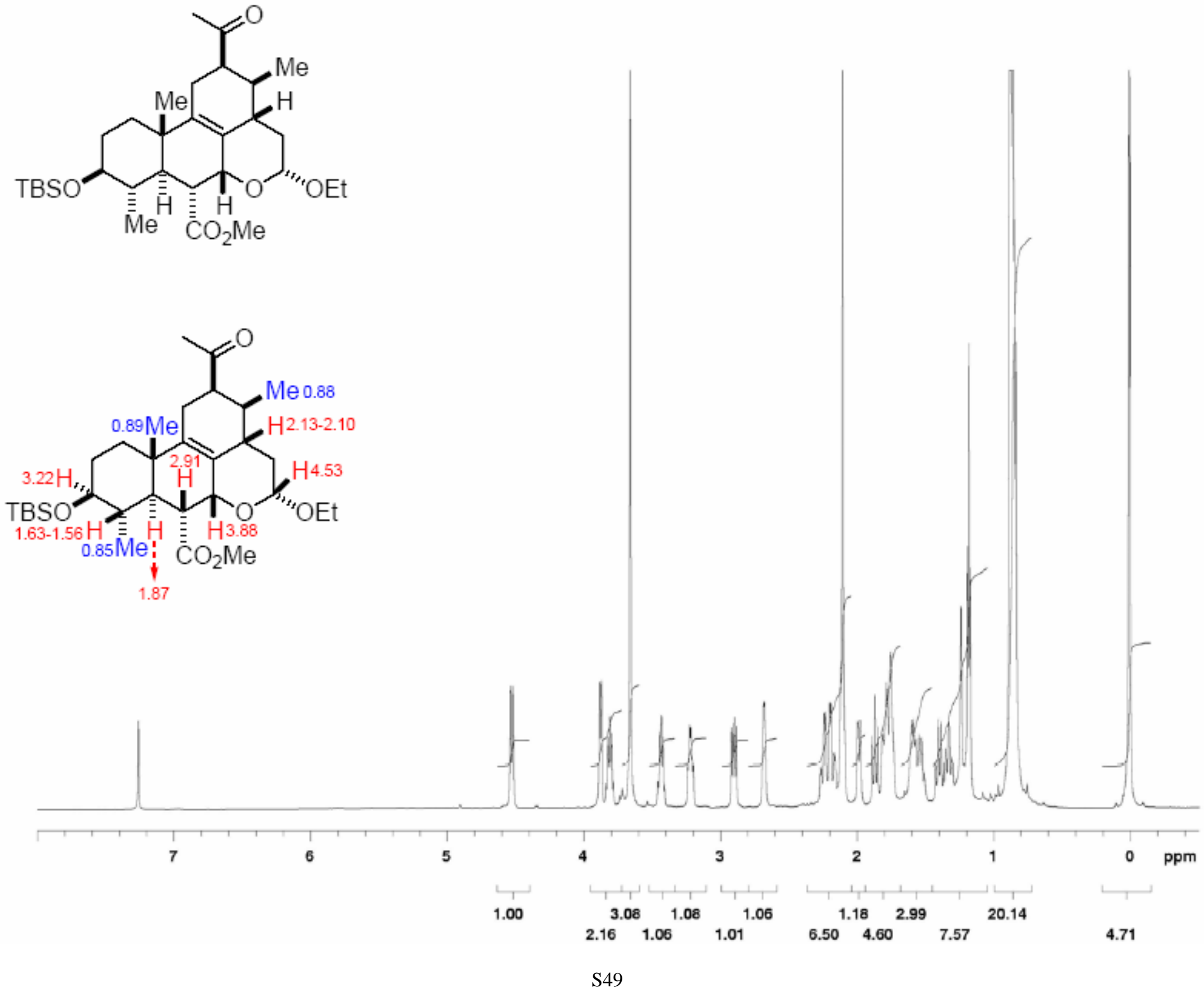
DQ-COSY of tetracyclic compound 28a

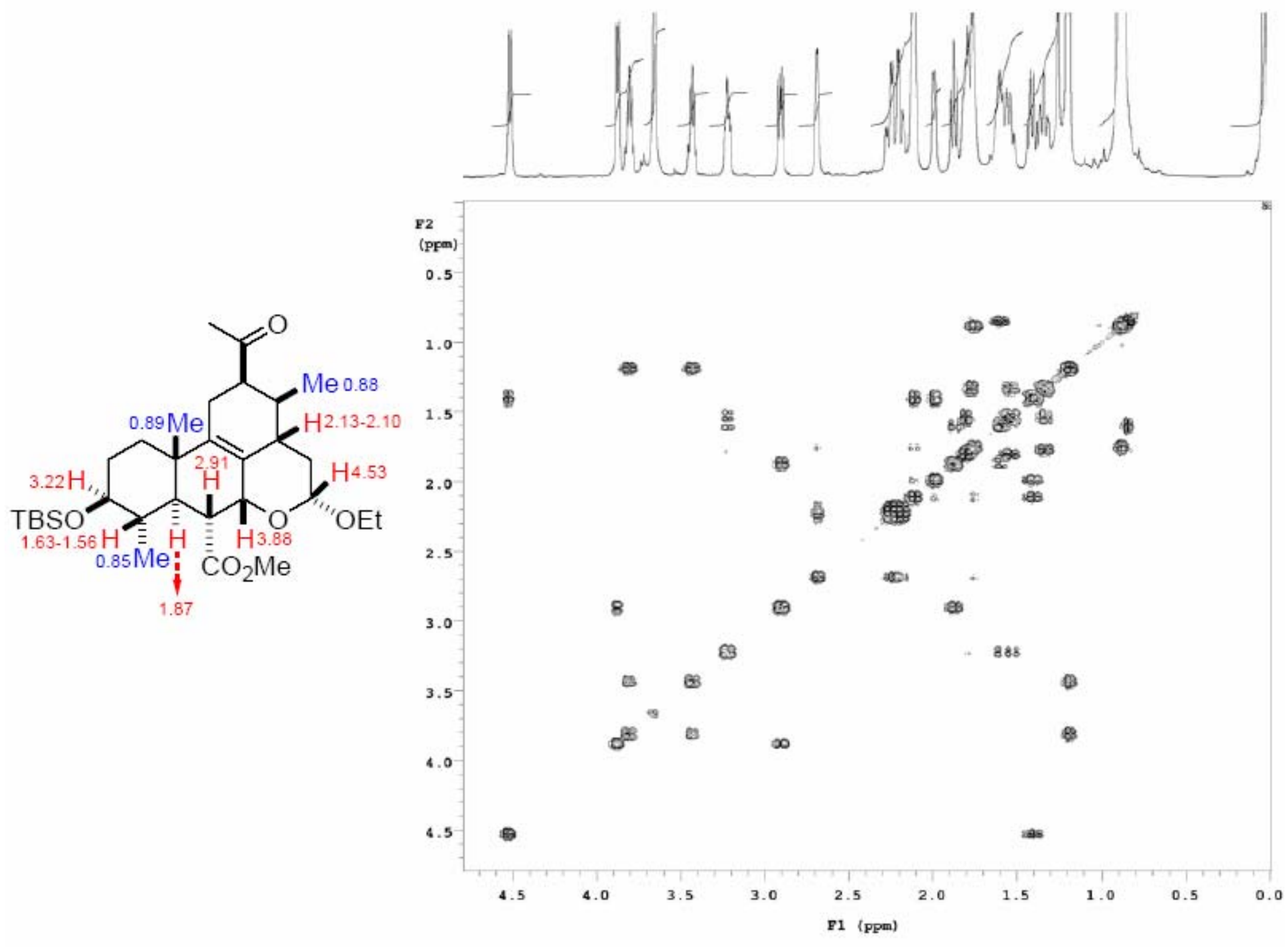



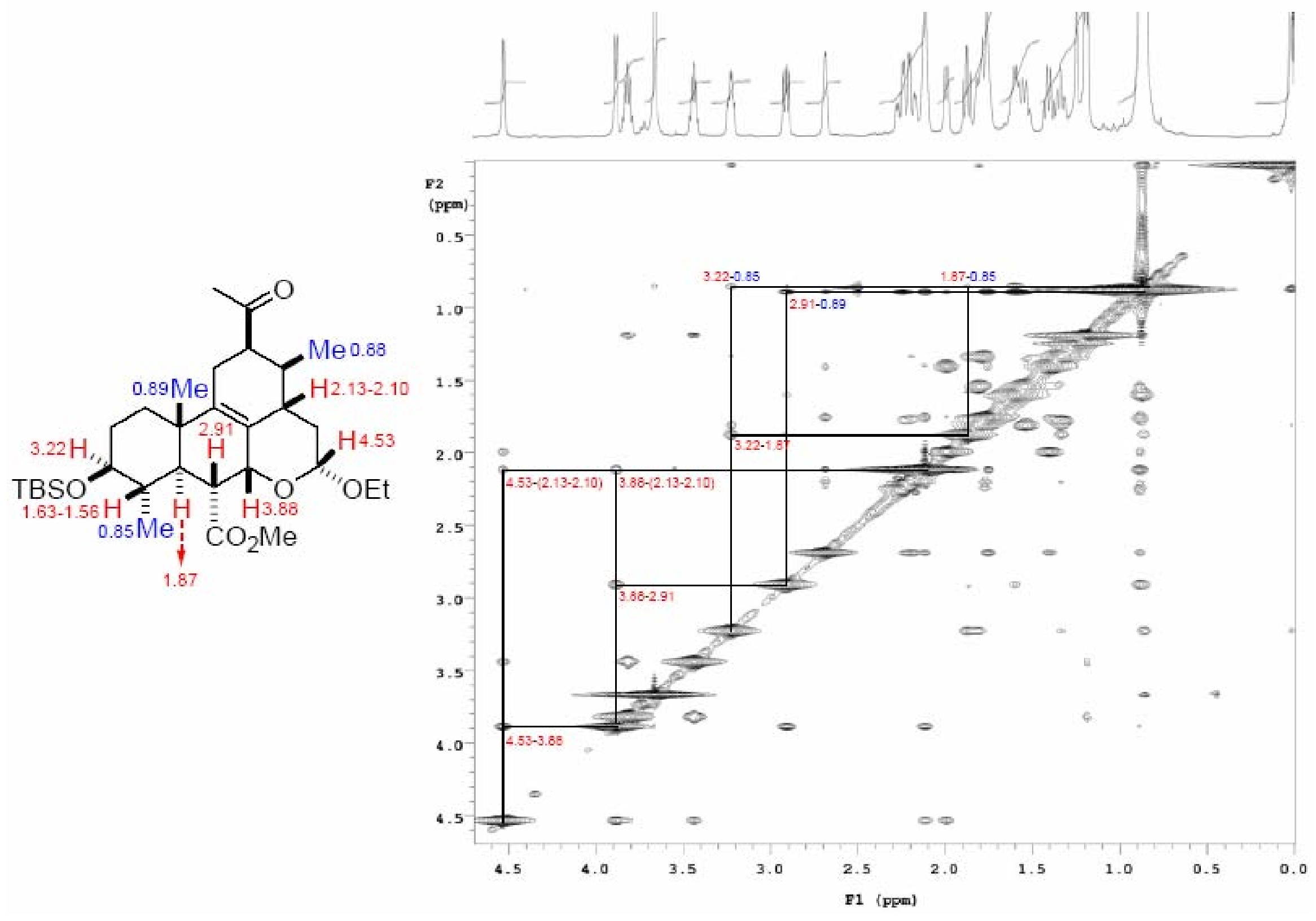


\section{Tetracyclic compound 28b}
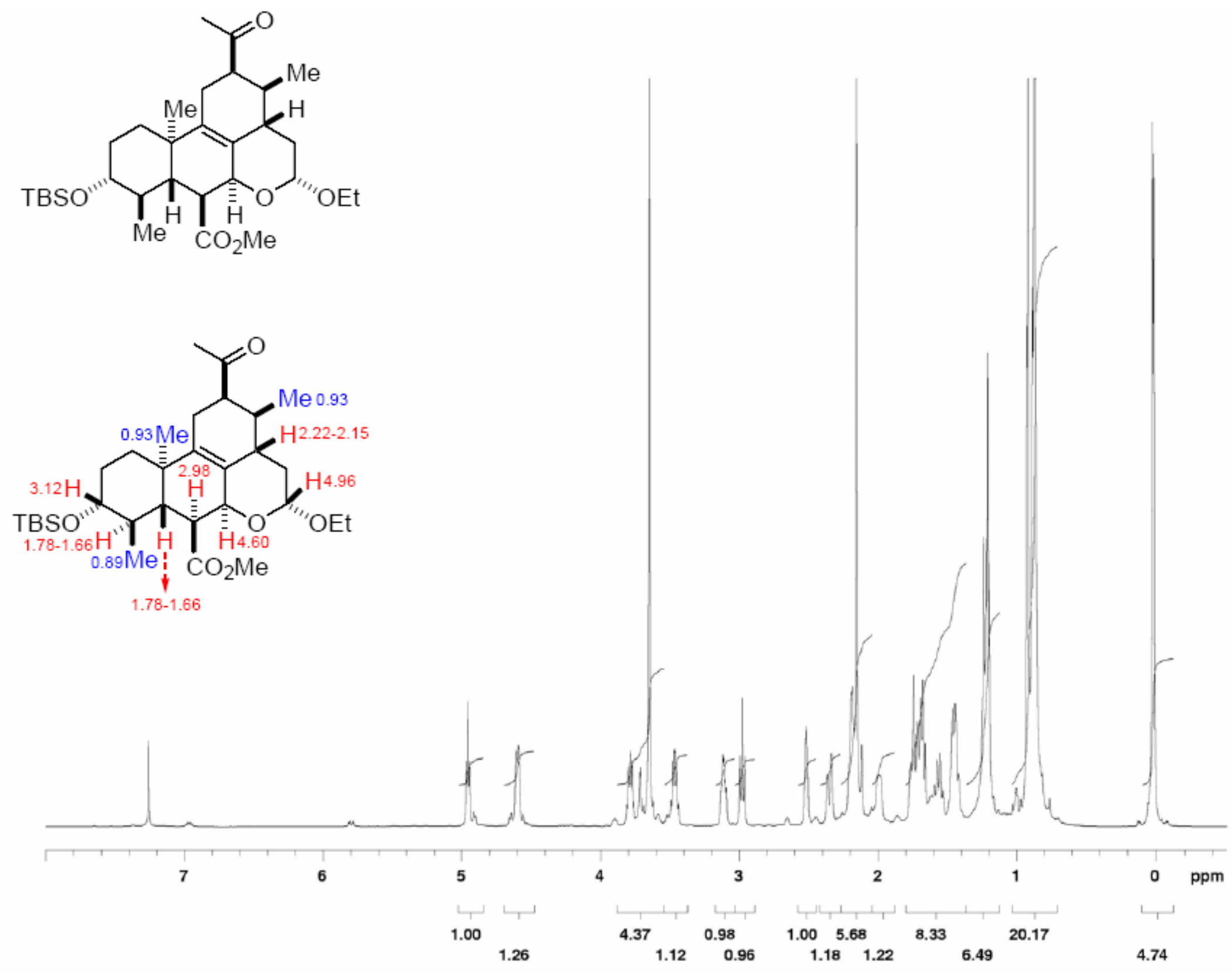
DQ-COSY of tetracyclic compound 28b

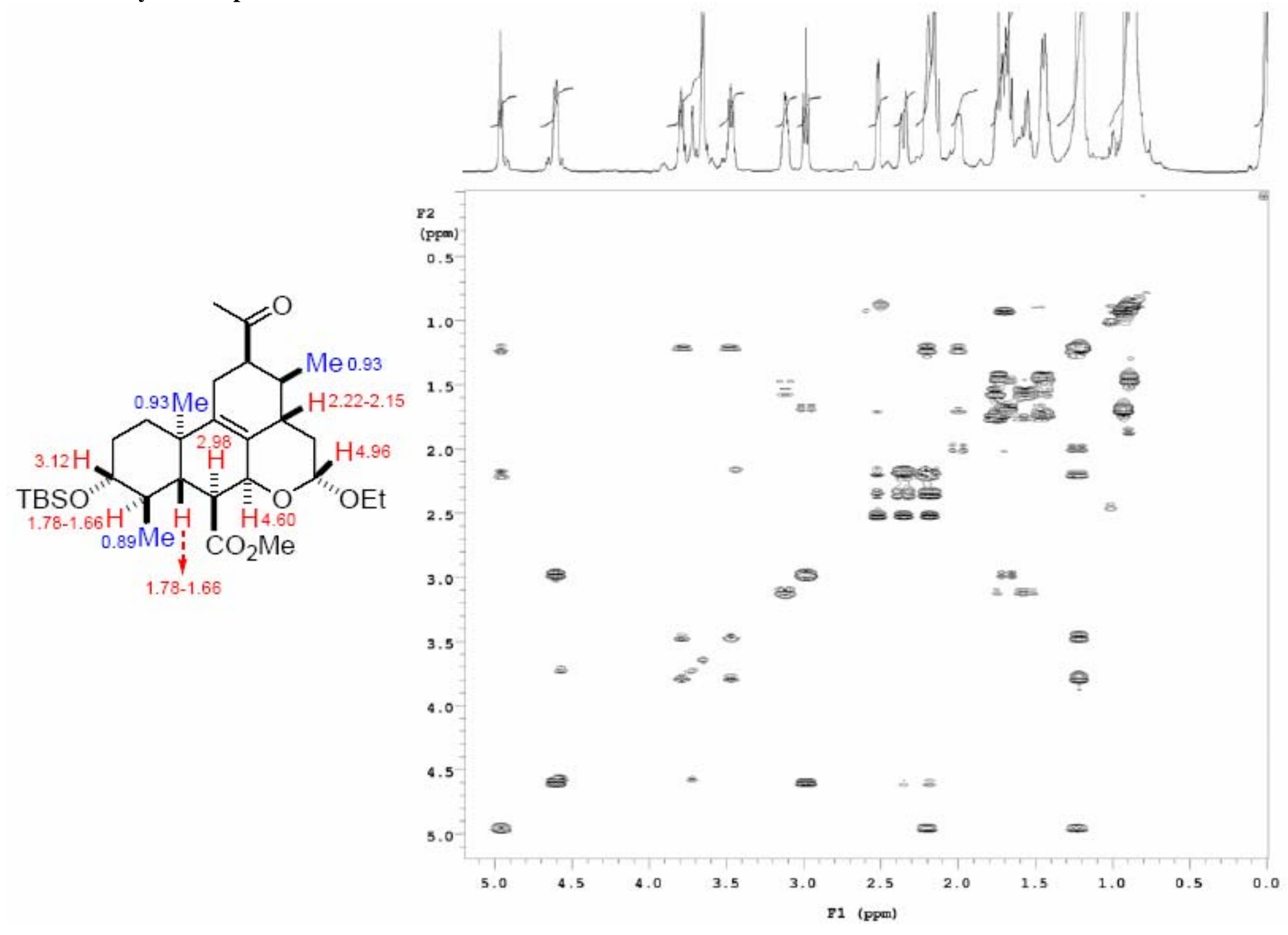




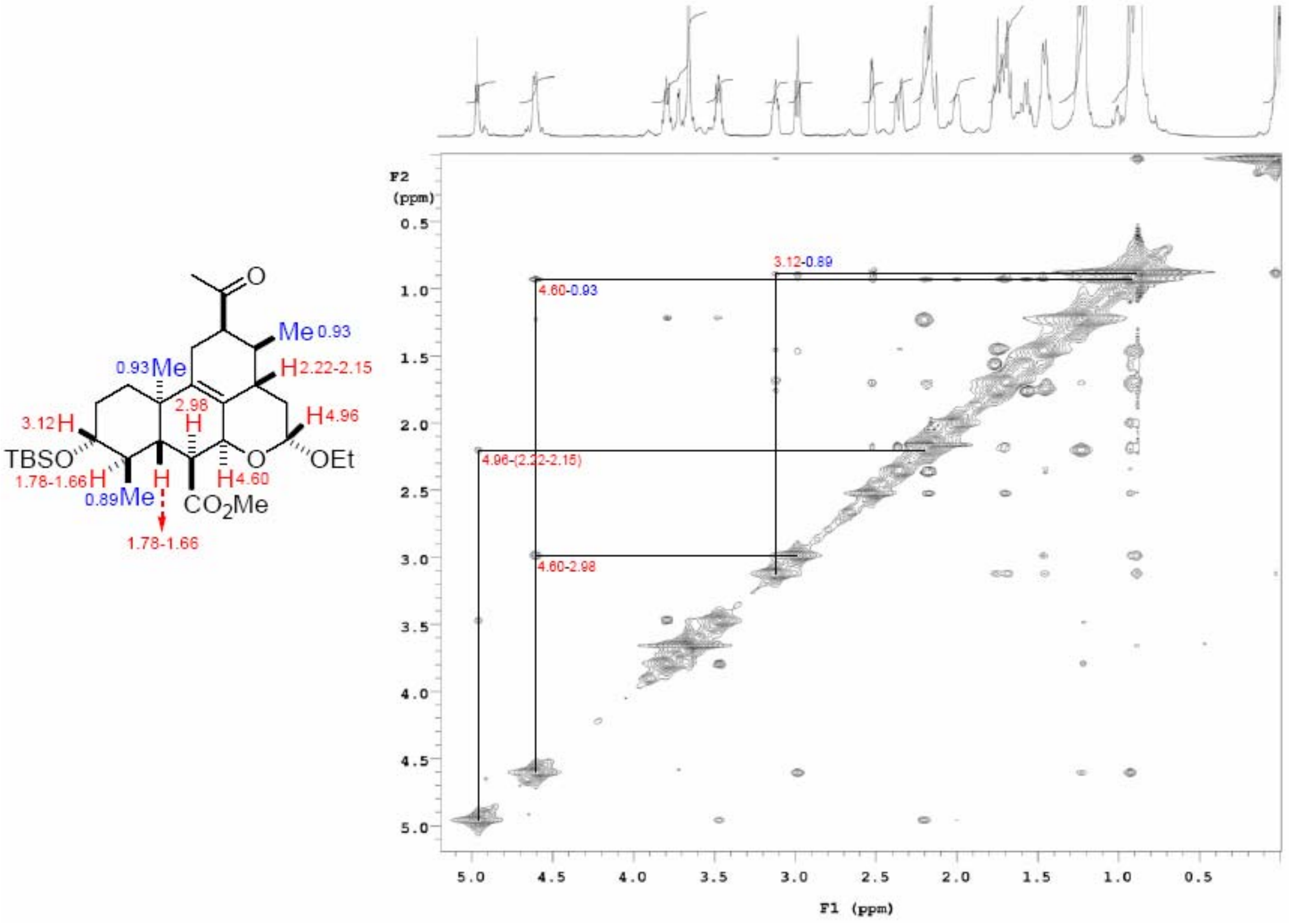




\section{Summary of X-Ray Crystallographic Data}

\section{Lactone 14a (CCDC 600917)}

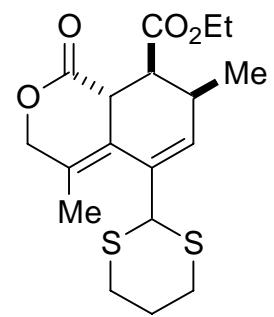

Crystals of $\mathrm{X}$ were grown by vapour diffusion using of hexanes on ethanol saturated solution at room temperature. Single crystals were coated with Paratone-N oil, mounted using a glass fibre and frozen in the cold nitrogen stream of the goniometer. A hemisphere of data was collected on a Bruker AXS P4/SMART 1000 diffractometer using $\omega$ and $\theta$ scans with a scan width of $0.3^{\circ}$ and $10 \mathrm{~s}$ exposure times. The detector distance was $5 \mathrm{~cm}$. The data were reduced $(\mathrm{SAINT})^{1}$ and corrected for absorption (SADABS). ${ }^{2}$ The structure was solved by direct methods and refined by fullmatrix least squares on $\mathrm{F}^{2}$ (SHELXTL). ${ }^{3}$ All non-hydrogen atoms were refined anisotropically. Hydrogen atoms were found in Fourier difference maps and refined isotropically.

(1) SAINT 6.02, 1997-1999, Bruker AXS, Inc., Madison, Wisconsin, USA.

(2) SADABS George Sheldrick, 1999, Bruker AXS, Inc., Madison,Wisconsin, USA.

(3) SHELXTL 5.1, George Sheldrick, 1997, Bruker AXS, Inc., Madison,Wisconsin, USA. 


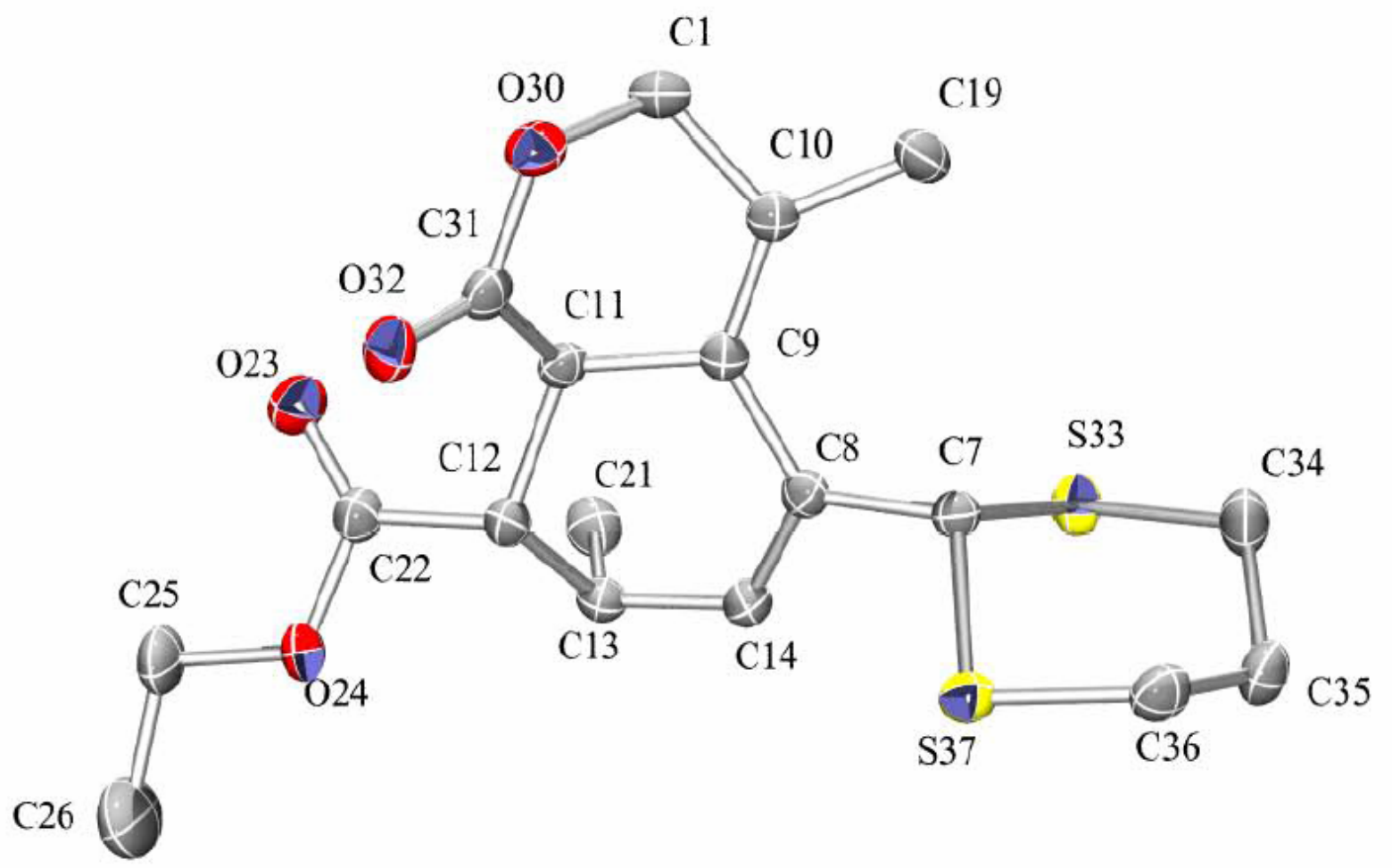

Table 1. Crystal data and structure refinement for SP2-127-1.

Identification code

Empirical formula

Formula weight

Temperature

Wavelength

Diffractometer used

Detector distance

Monochrometer used

Crystal size

Colour and habit

Crystal system

Space group

Unit cell dimensions

Volume

$\mathrm{Z}$

Density (calculated)

Absorption coefficient

$\mathrm{F}(000)$

Theta range for data collection
CS040170

C18 H24 O4 S2

368.49

198(1) K

$0.71073 \AA$

Bruker AXS P4/SMART 1000

$5 \mathrm{~cm}$

Graphite

$0.25 \times 0.4 \times 0.5 \mathrm{~mm}^{3}$

Colourless, plate

Triclinic

P-1

$\mathrm{a}=8.0381(7) \AA$

$\alpha=91.791(2)^{\circ}$

$\mathrm{b}=9.2203(9) \AA$

$\beta=94.580(2)^{\circ}$

$\mathrm{c}=12.4183(12) \AA$

$\gamma=95.953(2)^{\circ}$

911.75(15) $\AA^{3}$

2

$1.342 \mathrm{Mg} / \mathrm{m}^{3}$

$0.311 \mathrm{~mm}^{-1}$

392

1.65 to $27.48^{\circ}$ 
Completeness to theta $=27.48^{\circ}$

Scan type

Scan range

Exposure time

Index ranges

Standard reflections

Crystal stability

Reflections collected

Independent reflections

System used

Solution

Hydrogen atoms

Absorption correction

Min./Max. transmission ratio

Refinement method

Data / restraints / parameters

Goodness-of-fit on $\mathrm{F}^{2}$

Final R indices [I>2sigma(I)]

$\mathrm{R}$ indices (all data)

Largest/mean shift/esd

Largest diff. peak and hole
$95.1 \%$

$\omega$ and $\phi$

$0.3^{\circ}$

$10 \mathrm{~s}$

$-10 \leq \mathrm{h} \leq 10,-11 \leq \mathrm{k} \leq 11,-15 \leq \mathrm{l} \leq 16$

50 frames at beginning and end of data collection no decay

6361

$3972[\mathrm{R}(\mathrm{int})=0.0159]$

SHELXL 5.1

Direct methods

Found, refined isotropically

SADABS

0.883

Full-matrix least-squares on $\mathrm{F}^{2}$

3972 / 0 / 315

1.073

$\mathrm{R} 1=0.0353, \mathrm{wR} 2=0.0918$

$\mathrm{R} 1=0.0502, \mathrm{wR} 2=0.0989$

$0.000 / 0.000$

0.370 and -0.211 e. $\AA^{-3}$

$\mathrm{wR} 2=\left(\sum\left[\mathrm{w}\left(\mathrm{F}_{\mathrm{o}}{ }^{2}-\mathrm{F}_{\mathrm{c}}{ }^{2}\right)^{2}\right] / \sum\left[\mathrm{F}_{\mathrm{o}}{ }^{4}\right]\right)^{1 / 2}$

$\mathrm{R} 1=\sum|| \mathrm{F}_{\mathrm{o}}|-| \mathrm{F}_{\mathrm{c}}|| / \sum\left|\mathrm{F}_{\mathrm{o}}\right|$

Weight $=1 /\left[\sigma^{2}\left(\mathrm{~F}_{\mathrm{o}}^{2}\right)+(0.0534 * \mathrm{P})^{2}+(0.1439 * \mathrm{P})\right]$

where $\mathrm{P}=\left(\max \left(\mathrm{F}_{\mathrm{o}}^{2}, 0\right)+2 * \mathrm{~F}_{\mathrm{c}}^{2}\right) / 3$

Table 2. Atomic coordinates ( $\times 10^{4}$ ) and equivalent isotropic displacement parameters $\left(\AA^{2} \times 10^{3}\right)$ for SP2-127-1. U(eq) is defined as one third of the trace of the orthogonalized $U^{i j}$ tensor.

\begin{tabular}{lcccc}
\hline & $\mathrm{x}$ & $\mathrm{y}$ & $\mathrm{z}$ & $\mathrm{U}(\mathrm{eq})$ \\
\hline $\mathrm{C}(1)$ & $-1056(2)$ & $8818(2)$ & $6648(2)$ & $28(1)$ \\
$\mathrm{C}(7)$ & $2037(2)$ & $7252(2)$ & $3964(1)$ & $22(1)$ \\
$\mathrm{C}(8)$ & $2408(2)$ & $7216(2)$ & $5177(1)$ & $21(1)$ \\
$\mathrm{C}(9)$ & $1325(2)$ & $7867(2)$ & $5914(1)$ & $20(1)$ \\
$\mathrm{C}(10)$ & $-293(2)$ & $8105(2)$ & $5732(1)$ & $23(1)$ \\
$\mathrm{C}(11)$ & $2065(2)$ & $8224(2)$ & $7077(1)$ & $21(1)$ \\
$\mathrm{C}(12)$ & $3923(2)$ & $8048(2)$ & $7278(1)$ & $23(1)$ \\
$\mathrm{C}(13)$ & $4329(2)$ & $6607(2)$ & $6748(1)$ & $24(1)$
\end{tabular}




$\begin{array}{lrrrl}\mathrm{C}(14) & 3763(2) & 6609(2) & 5567(1) & 24(1) \\ \mathrm{C}(19) & -1520(2) & 7616(2) & 4790(2) & 30(1) \\ \mathrm{C}(21) & 3547(2) & 5256(2) & 7286(2) & 31(1) \\ \mathrm{C}(22) & 4456(2) & 8121(2) & 8472(1) & 26(1) \\ \mathrm{O}(23) & 3532(2) & 8069(2) & 9189(1) & 39(1) \\ \mathrm{O}(24) & 6130(2) & 8213(2) & 8641(1) & 35(1) \\ \mathrm{C}(25) & 6818(3) & 8287(3) & 9766(2) & 44(1) \\ \mathrm{C}(26) & 8643(4) & 8149(5) & 9773(2) & 75(1) \\ \mathrm{O}(30) & 66(1) & 9975(1) & 7215(1) & 30(1) \\ \mathrm{C}(31) & 1689(2) & 9735(2) & 7403(1) & 25(1) \\ \mathrm{O}(32) & 2686(2) & 10707(1) & 7790(1) & 33(1) \\ \mathrm{S}(33) & 1367(1) & 5416(1) & 3417(1) & 27(1) \\ \mathrm{C}(34) & 926(2) & 5857(2) & 2018(2) & 34(1) \\ \mathrm{C}(35) & 2453(2) & 6534(2) & 1497(2) & 33(1) \\ \mathrm{C}(36) & 3115(2) & 8039(2) & 1974(2) & 30(1) \\ \mathrm{S}(37) & 3904(1) & 8075(1) & 3385(1) & 26(1) \\ \end{array}$

Table 3. Bond lengths $[\AA]$ and angles $\left[^{\circ}\right]$ for SP2-127-1.

\begin{tabular}{ll}
\hline $\mathrm{C}(1)-\mathrm{O}(30)$ & $1.449(2)$ \\
$\mathrm{C}(1)-\mathrm{C}(10)$ & $1.500(2)$ \\
$\mathrm{C}(1)-\mathrm{H}(1 \mathrm{~A})$ & $0.95(2)$ \\
$\mathrm{C}(1)-\mathrm{H}(1 \mathrm{~B})$ & $0.92(2)$ \\
$\mathrm{C}(7)-\mathrm{C}(8)$ & $1.514(2)$ \\
$\mathrm{C}(7)-\mathrm{S}(33)$ & $1.8156(17)$ \\
$\mathrm{C}(7)-\mathrm{S}(37)$ & $1.8205(16)$ \\
$\mathrm{C}(7)-\mathrm{H}(7)$ & $0.961(19)$ \\
$\mathrm{C}(8)-\mathrm{C}(14)$ & $1.339(2)$ \\
$\mathrm{C}(8)-\mathrm{C}(9)$ & $1.469(2)$ \\
$\mathrm{C}(9)-\mathrm{C}(10)$ & $1.343(2)$ \\
$\mathrm{C}(9)-\mathrm{C}(11)$ & $1.530(2)$ \\
$\mathrm{C}(10)-\mathrm{C}(19)$ & $1.496(2)$ \\
$\mathrm{C}(11)-\mathrm{C}(31)$ & $1.506(2)$ \\
$\mathrm{C}(11)-\mathrm{C}(12)$ & $1.521(2)$ \\
$\mathrm{C}(11)-\mathrm{H}(11)$ & $0.96(2)$ \\
$\mathrm{C}(12)-\mathrm{C}(22)$ & $1.507(2)$ \\
$\mathrm{C}(12)-\mathrm{C}(13)$ & $1.541(2)$ \\
$\mathrm{C}(12)-\mathrm{H}(12)$ & $0.95(2)$ \\
$\mathrm{C}(13)-\mathrm{C}(14)$ & $1.500(2)$ \\
$\mathrm{C}(13)-\mathrm{C}(21)$ & $1.533(3)$
\end{tabular}




\begin{tabular}{|c|c|}
\hline C(13)-H(13) & $0.972(19)$ \\
\hline C(14)-H(14) & $0.96(2)$ \\
\hline C(19)-H(19C) & $0.96(2)$ \\
\hline C(19)-H(19A) & $0.99(2)$ \\
\hline C(19)-H(19B) & $0.92(3)$ \\
\hline C(21)-H(21A) & $0.98(2)$ \\
\hline C(21)-H(21B) & $0.97(2)$ \\
\hline C(21)-H(21C) & $0.99(2)$ \\
\hline $\mathrm{C}(22)-\mathrm{O}(23)$ & $1.203(2)$ \\
\hline $\mathrm{C}(22)-\mathrm{O}(24)$ & $1.339(2)$ \\
\hline $\mathrm{O}(24)-\mathrm{C}(25)$ & $1.457(2)$ \\
\hline$C(25)-C(26)$ & $1.485(3)$ \\
\hline C(25)-H(25B) & $1.19(2)$ \\
\hline $\mathrm{C}(25)-\mathrm{H}(25 \mathrm{~A})$ & $0.93(2)$ \\
\hline $\mathrm{C}(26)-\mathrm{H}(26 \mathrm{~A})$ & $1.00(3)$ \\
\hline C(26)-H(26B) & $1.07(5)$ \\
\hline C(26)-H(26C) & $1.14(6)$ \\
\hline $\mathrm{O}(30)-\mathrm{C}(31)$ & $1.349(2)$ \\
\hline $\mathrm{C}(31)-\mathrm{O}(32)$ & $1.199(2)$ \\
\hline S(33)-C(34) & $1.8115(19)$ \\
\hline C(34)-C(35) & $1.520(3)$ \\
\hline C(34)-H(34B) & $0.95(2)$ \\
\hline C(34)-H(34A) & $1.01(2)$ \\
\hline C(35)-C(36) & $1.518(3)$ \\
\hline C(35)-H(35A) & $0.96(2)$ \\
\hline C(35)-H(35B) & $1.00(2)$ \\
\hline C(36)-S(37) & $1.8135(19)$ \\
\hline C(36)-H(36B) & $0.93(2)$ \\
\hline $\mathrm{C}(36)-\mathrm{H}(36 \mathrm{~A})$ & $0.99(2)$ \\
\hline $\mathrm{O}(30)-\mathrm{C}(1)-\mathrm{C}(10)$ & 113.17(13) \\
\hline $\mathrm{O}(30)-\mathrm{C}(1)-\mathrm{H}(1 \mathrm{~A})$ & 106.1(12) \\
\hline $\mathrm{C}(10)-\mathrm{C}(1)-\mathrm{H}(1 \mathrm{~A})$ & $111.4(12)$ \\
\hline $\mathrm{O}(30)-\mathrm{C}(1)-\mathrm{H}(1 \mathrm{~B})$ & $110.3(12)$ \\
\hline $\mathrm{C}(10)-\mathrm{C}(1)-\mathrm{H}(1 \mathrm{~B})$ & $107.4(13)$ \\
\hline $\mathrm{H}(1 \mathrm{~A})-\mathrm{C}(1)-\mathrm{H}(1 \mathrm{~B})$ & $108.3(17)$ \\
\hline C(8)-C(7)-S(33) & 109.49(11) \\
\hline $\mathrm{C}(8)-\mathrm{C}(7)-\mathrm{S}(37)$ & $108.57(10)$ \\
\hline S(33)-C(7)-S(37) & $112.56(9)$ \\
\hline $\mathrm{C}(8)-\mathrm{C}(7)-\mathrm{H}(7)$ & $112.3(11)$ \\
\hline $\mathrm{S}(33)-\mathrm{C}(7)-\mathrm{H}(7)$ & $107.9(11)$ \\
\hline S(37)-C(7)-H(7) & $106.0(11)$ \\
\hline
\end{tabular}




\begin{tabular}{|c|c|}
\hline $\mathrm{C}(14)-\mathrm{C}(8)-\mathrm{C}(9)$ & $120.46(15)$ \\
\hline $\mathrm{C}(14)-\mathrm{C}(8)-\mathrm{C}(7)$ & 118.92(15) \\
\hline C(9)-C(8)-C(7) & $120.61(14)$ \\
\hline C(10)-C(9)-C(8) & $128.76(15)$ \\
\hline C(10)-C(9)-C(11) & $114.21(14)$ \\
\hline $\mathrm{C}(8)-\mathrm{C}(9)-\mathrm{C}(11)$ & $116.84(13)$ \\
\hline C(9)-C(10)-C(19) & 129.39(16) \\
\hline $\mathrm{C}(9)-\mathrm{C}(10)-\mathrm{C}(1)$ & $116.28(15)$ \\
\hline C(19)-C(10)-C(1) & 113.96(14) \\
\hline C(31)-C(11)-C(12) & $111.75(14)$ \\
\hline $\mathrm{C}(31)-\mathrm{C}(11)-\mathrm{C}(9)$ & 108.64(13) \\
\hline $\mathrm{C}(12)-\mathrm{C}(11)-\mathrm{C}(9)$ & $114.77(13)$ \\
\hline $\mathrm{C}(31)-\mathrm{C}(11)-\mathrm{H}(11)$ & $104.4(12)$ \\
\hline $\mathrm{C}(12)-\mathrm{C}(11)-\mathrm{H}(11)$ & 108.6(12) \\
\hline C(9)-C(11)-H(11) & 108.1(12) \\
\hline $\mathrm{C}(22)-\mathrm{C}(12)-\mathrm{C}(11)$ & $110.86(14)$ \\
\hline C(22)-C(12)-C(13) & $110.73(14)$ \\
\hline$C(11)-C(12)-C(13)$ & $110.73(13)$ \\
\hline $\mathrm{C}(22)-\mathrm{C}(12)-\mathrm{H}(12)$ & $107.4(12)$ \\
\hline $\mathrm{C}(11)-\mathrm{C}(12)-\mathrm{H}(12)$ & $107.2(12)$ \\
\hline $\mathrm{C}(13)-\mathrm{C}(12)-\mathrm{H}(12)$ & 109.8(12) \\
\hline C(14)-C(13)-C(21) & $112.00(14)$ \\
\hline$C(14)-C(13)-C(12)$ & $107.76(14)$ \\
\hline $\mathrm{C}(21)-\mathrm{C}(13)-\mathrm{C}(12)$ & $112.87(14)$ \\
\hline C(14)-C(13)-H(13) & $107.7(11)$ \\
\hline $\mathrm{C}(21)-\mathrm{C}(13)-\mathrm{H}(13)$ & 108.6(11) \\
\hline $\mathrm{C}(12)-\mathrm{C}(13)-\mathrm{H}(13)$ & $107.6(11)$ \\
\hline $\mathrm{C}(8)-\mathrm{C}(14)-\mathrm{C}(13)$ & $123.70(15)$ \\
\hline $\mathrm{C}(8)-\mathrm{C}(14)-\mathrm{H}(14)$ & $118.3(12)$ \\
\hline $\mathrm{C}(13)-\mathrm{C}(14)-\mathrm{H}(14)$ & $117.9(12)$ \\
\hline C(10)-C(19)-H(19C) & $108.9(13)$ \\
\hline $\mathrm{C}(10)-\mathrm{C}(19)-\mathrm{H}(19 \mathrm{~A})$ & 113.8(13) \\
\hline H(19C)-C(19)-H(19A) & 108.6(18) \\
\hline C(10)-C(19)-H(19B) & 113.3(15) \\
\hline H(19C)-C(19)-H(19B) & 104.2(19) \\
\hline H(19A)-C(19)-H(19B) & $107(2)$ \\
\hline $\mathrm{C}(13)-\mathrm{C}(21)-\mathrm{H}(21 \mathrm{~A})$ & $113.3(12)$ \\
\hline C(13)-C(21)-H(21B) & 109.6(13) \\
\hline $\mathrm{H}(21 \mathrm{~A})-\mathrm{C}(21)-\mathrm{H}(21 \mathrm{~B})$ & $106.8(17)$ \\
\hline $\mathrm{C}(13)-\mathrm{C}(21)-\mathrm{H}(21 \mathrm{C})$ & $113.2(12)$ \\
\hline$H(21 A)-C(21)-H(21 C)$ & $103.2(17)$ \\
\hline $\mathrm{H}(21 \mathrm{~B})-\mathrm{C}(21)-\mathrm{H}(21 \mathrm{C})$ & $110.4(18)$ \\
\hline
\end{tabular}




\begin{tabular}{|c|c|}
\hline $\mathrm{O}(23)-\mathrm{C}(22)-\mathrm{O}(24)$ & $123.50(16)$ \\
\hline $\mathrm{O}(23)-\mathrm{C}(22)-\mathrm{C}(12)$ & $125.92(15)$ \\
\hline $\mathrm{O}(24)-\mathrm{C}(22)-\mathrm{C}(12)$ & $110.56(14)$ \\
\hline $\mathrm{C}(22)-\mathrm{O}(24)-\mathrm{C}(25)$ & 116.31(14) \\
\hline $\mathrm{O}(24)-\mathrm{C}(25)-\mathrm{C}(26)$ & $107.56(19)$ \\
\hline $\mathrm{O}(24)-\mathrm{C}(25)-\mathrm{H}(25 \mathrm{~B})$ & $110.6(12)$ \\
\hline $\mathrm{C}(26)-\mathrm{C}(25)-\mathrm{H}(25 \mathrm{~B})$ & $87.4(11)$ \\
\hline $\mathrm{O}(24)-\mathrm{C}(25)-\mathrm{H}(25 \mathrm{~A})$ & $109.7(14)$ \\
\hline $\mathrm{C}(26)-\mathrm{C}(25)-\mathrm{H}(25 \mathrm{~A})$ & $116.9(14)$ \\
\hline $\mathrm{H}(25 \mathrm{~B})-\mathrm{C}(25)-\mathrm{H}(25 \mathrm{~A})$ & 122.4(19) \\
\hline C(25)-C(26)-H(26A) & 110.7(18) \\
\hline C(25)-C(26)-H(26B) & $105(2)$ \\
\hline $\mathrm{H}(26 \mathrm{~A})-\mathrm{C}(26)-\mathrm{H}(26 \mathrm{~B})$ & 110(3) \\
\hline $\mathrm{C}(25)-\mathrm{C}(26)-\mathrm{H}(26 \mathrm{C})$ & 114(3) \\
\hline $\mathrm{H}(26 \mathrm{~A})-\mathrm{C}(26)-\mathrm{H}(26 \mathrm{C})$ & 104(3) \\
\hline H(26B)-C(26)-H(26C) & 113(3) \\
\hline C(31)-O(30)-C(1) & $117.06(13)$ \\
\hline $\mathrm{O}(32)-\mathrm{C}(31)-\mathrm{O}(30)$ & $119.17(16)$ \\
\hline $\mathrm{O}(32)-\mathrm{C}(31)-\mathrm{C}(11)$ & $126.19(16)$ \\
\hline $\mathrm{O}(30)-\mathrm{C}(31)-\mathrm{C}(11)$ & $114.64(14)$ \\
\hline C(34)-S(33)-C(7) & 98.15(9) \\
\hline C(35)-C(34)-S(33) & $113.75(13)$ \\
\hline C(35)-C(34)-H(34B) & $111.8(14)$ \\
\hline S(33)-C(34)-H(34B) & $103.5(14)$ \\
\hline $\mathrm{C}(35)-\mathrm{C}(34)-\mathrm{H}(34 \mathrm{~A})$ & $110.8(13)$ \\
\hline S(33)-C(34)-H(34A) & $108.3(13)$ \\
\hline $\mathrm{H}(34 \mathrm{~B})-\mathrm{C}(34)-\mathrm{H}(34 \mathrm{~A})$ & $108.5(18)$ \\
\hline C(36)-C(35)-C(34) & $113.23(16)$ \\
\hline C(36)-C(35)-H(35A) & 108.9(13) \\
\hline $\mathrm{C}(34)-\mathrm{C}(35)-\mathrm{H}(35 \mathrm{~A})$ & 109.7(13) \\
\hline C(36)-C(35)-H(35B) & $110.3(11)$ \\
\hline C(34)-C(35)-H(35B) & $111.5(12)$ \\
\hline H(35A)-C(35)-H(35B) & $102.7(17)$ \\
\hline C(35)-C(36)-S(37) & $114.63(13)$ \\
\hline C(35)-C(36)-H(36B) & $111.0(13)$ \\
\hline S(37)-C(36)-H(36B) & 104.1(13) \\
\hline $\mathrm{C}(35)-\mathrm{C}(36)-\mathrm{H}(36 \mathrm{~A})$ & $110.4(12)$ \\
\hline S(37)-C(36)-H(36A) & $108.3(12)$ \\
\hline $\mathrm{H}(36 \mathrm{~B})-\mathrm{C}(36)-\mathrm{H}(36 \mathrm{~A})$ & $108.0(18)$ \\
\hline C(36)-S(37)-C(7) & $99.64(8)$ \\
\hline
\end{tabular}

Symmetry transformations used to generate equivalent atoms: 
Table 4. Anisotropic displacement parameters $\left(\AA^{2} \times 10^{3}\right)$ for SP2-127-1. The anisotropic displacement factor exponent takes the form: $-2 \pi^{2}\left[h^{2} a^{* 2} U^{11}+\ldots+2 h k a^{*} b^{*} U^{12}\right]$

\begin{tabular}{|c|c|c|c|c|c|c|}
\hline & $\mathrm{U}^{11}$ & $\mathrm{U}^{22}$ & $\mathrm{U}^{33}$ & $\mathrm{U}^{23}$ & $\mathrm{U}^{13}$ & $\mathrm{U}^{12}$ \\
\hline$C(1)$ & $21(1)$ & $31(1)$ & $35(1)$ & $2(1)$ & $7(1)$ & $5(1)$ \\
\hline $\mathrm{C}(7)$ & $20(1)$ & 24(1) & $22(1)$ & $1(1)$ & $3(1)$ & $3(1)$ \\
\hline C(8) & $20(1)$ & 21(1) & $22(1)$ & $0(1)$ & $3(1)$ & $0(1)$ \\
\hline C(9) & 21(1) & 19(1) & $22(1)$ & $2(1)$ & $3(1)$ & $1(1)$ \\
\hline$C(10)$ & $22(1)$ & $22(1)$ & $25(1)$ & $4(1)$ & $4(1)$ & $2(1)$ \\
\hline C(11) & $21(1)$ & $24(1)$ & 20(1) & $2(1)$ & $5(1)$ & $3(1)$ \\
\hline $\mathrm{C}(12)$ & 21(1) & $27(1)$ & 20(1) & $1(1)$ & 2(1) & $2(1)$ \\
\hline C(13) & 19(1) & $30(1)$ & 25(1) & $-1(1)$ & $1(1)$ & $7(1)$ \\
\hline C(14) & 23(1) & $27(1)$ & 23(1) & $-2(1)$ & $3(1)$ & $5(1)$ \\
\hline C(19) & $20(1)$ & $42(1)$ & 28(1) & $6(1)$ & $0(1)$ & $2(1)$ \\
\hline$C(21)$ & 33(1) & $28(1)$ & 33(1) & $2(1)$ & $-2(1)$ & $8(1)$ \\
\hline C(22) & $28(1)$ & $27(1)$ & 23(1) & $1(1)$ & $2(1)$ & $4(1)$ \\
\hline $\mathrm{O}(23)$ & $35(1)$ & $61(1)$ & 24(1) & $6(1)$ & $7(1)$ & $10(1)$ \\
\hline O(24) & $27(1)$ & $57(1)$ & 21(1) & $-5(1)$ & $-3(1)$ & $5(1)$ \\
\hline C(25) & $41(1)$ & $69(2)$ & $22(1)$ & $-8(1)$ & $-8(1)$ & $16(1)$ \\
\hline C(26) & $52(2)$ & 131(3) & $42(2)$ & $-14(2)$ & $-14(1)$ & $32(2)$ \\
\hline $\mathrm{O}(30)$ & $27(1)$ & $28(1)$ & $35(1)$ & $-3(1)$ & $5(1)$ & $9(1)$ \\
\hline C(31) & $28(1)$ & $30(1)$ & 19(1) & $3(1)$ & $6(1)$ & $6(1)$ \\
\hline $\mathrm{O}(32)$ & $37(1)$ & $30(1)$ & $31(1)$ & $-6(1)$ & $1(1)$ & $3(1)$ \\
\hline S(33) & $32(1)$ & $24(1)$ & 25(1) & $1(1)$ & $1(1)$ & $-1(1)$ \\
\hline C(34) & $37(1)$ & $39(1)$ & 23(1) & $-2(1)$ & $-1(1)$ & $-2(1)$ \\
\hline C(35) & $38(1)$ & $42(1)$ & 21(1) & $0(1)$ & $5(1)$ & $6(1)$ \\
\hline C(36) & $31(1)$ & $36(1)$ & $26(1)$ & $7(1)$ & $9(1)$ & $5(1)$ \\
\hline S(37) & 23(1) & $28(1)$ & $27(1)$ & $2(1)$ & $6(1)$ & $0(1)$ \\
\hline
\end{tabular}

Table 5. Hydrogen coordinates ( $\times 10^{4}$ ) and isotropic displacement parameters $\left(\AA^{2} \times 10^{3}\right)$ for SP2-127-1.

\begin{tabular}{lcccc}
\hline & $\mathrm{x}$ & $\mathrm{y}$ & $\mathrm{z}$ & $\mathrm{U}(\mathrm{eq})$ \\
\hline $\mathrm{H}(1 \mathrm{~A})$ & & & & \\
$\mathrm{H}(1 \mathrm{~B})$ & $-2030(30)$ & $9250(20)$ & $6401(16)$ & $31(5)$ \\
$\mathrm{H}(7)$ & $-1360(20)$ & $8100(20)$ & $7114(17)$ & $32(5)$ \\
& $1160(20)$ & $7850(20)$ & $3767(15)$ & $25(5)$
\end{tabular}




\begin{tabular}{|c|c|c|c|c|}
\hline $\mathrm{H}(11)$ & $1460(20)$ & $7600(20)$ & $7544(16)$ & $31(5)$ \\
\hline $\mathrm{H}(12)$ & $4530(20)$ & $8850(20)$ & 6966(16) & $32(5)$ \\
\hline $\mathrm{H}(13)$ & $5540(20)$ & $6610(20)$ & $6813(15)$ & $27(5)$ \\
\hline $\mathrm{H}(14)$ & $4430(20)$ & $6170(20)$ & $5063(16)$ & $34(5)$ \\
\hline H(19A) & $-1180(30)$ & $6810(30)$ & 4342(19) & $50(6)$ \\
\hline $\mathrm{H}(19 B)$ & $-1740(30)$ & $8350(30)$ & $4340(20)$ & $49(7)$ \\
\hline H(19C) & $-2580(30)$ & $7310(20)$ & $5050(18)$ & $42(6)$ \\
\hline $\mathrm{H}(21 \mathrm{~A})$ & $2320(30)$ & $5160(20)$ & 7226(16) & $34(5)$ \\
\hline $\mathrm{H}(21 \mathrm{~B})$ & $3880(30)$ & $4380(20)$ & 6942(17) & $38(6)$ \\
\hline $\mathrm{H}(21 \mathrm{C})$ & $3840(30)$ & $5270(20)$ & 8074(18) & $40(6)$ \\
\hline $\mathrm{H}(25 \mathrm{~A})$ & $6510(30)$ & $9110(30)$ & 10118(18) & $45(6)$ \\
\hline $\mathrm{H}(25 \mathrm{~B})$ & $6670(30)$ & $7110(30)$ & 10138(19) & $56(7)$ \\
\hline $\mathrm{H}(26 \mathrm{~A})$ & $9200(40)$ & $8300(30)$ & 10530(30) & $93(10)$ \\
\hline $\mathrm{H}(26 \mathrm{~B})$ & $8710(50)$ & $7060(50)$ & $9470(30)$ & $145(16)$ \\
\hline $\mathrm{H}(26 \mathrm{C})$ & $9350(60)$ & $9010(60)$ & $9290(40)$ & $190(20)$ \\
\hline $\mathrm{H}(34 \mathrm{~A})$ & $10(30)$ & $6520(20)$ & 1984(18) & $44(6)$ \\
\hline H(34B) & $490(30)$ & $4940(30)$ & $1676(18)$ & $48(6)$ \\
\hline $\mathrm{H}(35 \mathrm{~A})$ & $2180(30)$ & $6600(20)$ & 738(19) & $45(6)$ \\
\hline $\mathrm{H}(35 \mathrm{~B})$ & $3370(30)$ & $5880(20)$ & 1523(16) & $35(5)$ \\
\hline $\mathrm{H}(36 \mathrm{~A})$ & $2240(30)$ & $8710(20)$ & 1898(16) & $36(5)$ \\
\hline $\mathrm{H}(36 \mathrm{~B})$ & $4030(30)$ & $8430(20)$ & $1625(17)$ & $41(6)$ \\
\hline
\end{tabular}




\section{Lactone 14b (CCDC 600922)}<smiles>CC(=O)C1C(C)C=C(C2SCCCS2)C2=C(C)COC(=O)C21</smiles>

Experimental : The crystals were grown by vapor diffusion of hexanes on a dichloromethane saturated solution at room temperature. One single crystal was mounted using a glass fiber on the goniometer. Data were collected on an EnrafNonius CAD-4 automatic diffractometer at the Universite de Sherbrooke using $\omega / 2 \theta$ scans at 293(2) K. The DIFRAC ${ }^{(1)}$ program was used for centering, indexing, and data collection. Two standard reflections were measured every 100 reflections, no intensity decay was observed during data collection. The data were corrected for absorption by empirical methods based on psi scans and reduced with the NRCVAX ${ }^{(2)}$ programs. They were solved using SHELXS-97 ${ }^{(3)}$ and refined by full-matrix least squares on $\mathrm{F}^{2}$ with SHELXL-97 ${ }^{(4)}$. The non-hydrogen atoms were refined anisotropically. The hydrogen atoms were placed at idealized calculated geometric position and refined isotropically using a riding model.

(1) H.D. Flack, E. Blanc and D. Schwarzenbach (1992), J. Appl. Cryst., 25, 455-459.

(2) E.J. Gabe, Y. Le Page, J.-P. Charland, F.L. Lee, and P.S. White, (1989) J. Appl. Cryst., 22, 384-387.

(3) G. M. Sheldrick, SHELXS-97, G.M. Sheldrick, University of Göttingen, Germany, 1997, Release 97-2.

(4) G. M. Sheldrick, SHELXL-97, G.M. Sheldrick, University of Göttingen, Germany, 1997, Release 97-2. 


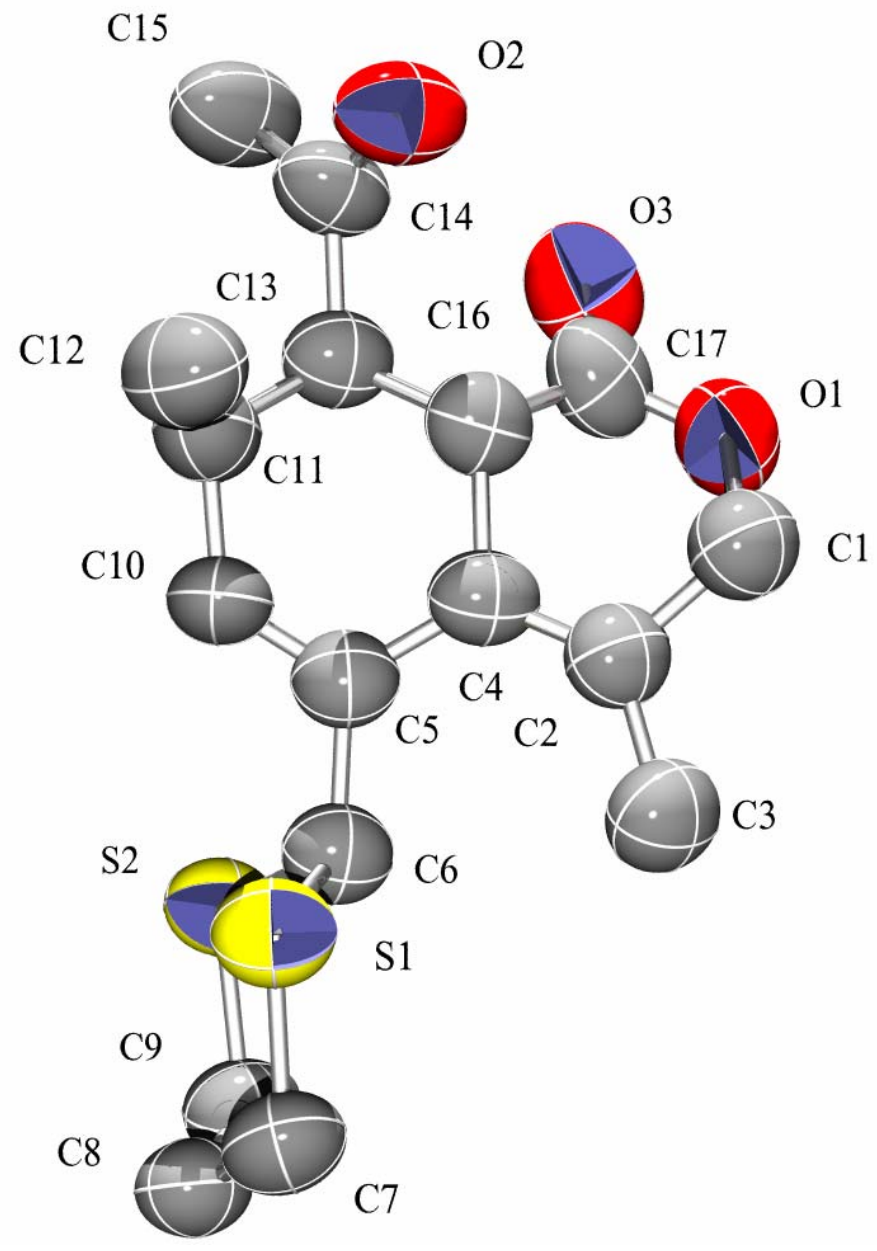

Table 1. Crystal data and structure refinement for sp5010.

Identification code

Empirical formula

Formula weight

Temperature

Wavelength

Crystal system

Space group

Unit cell dimensions

Volume

$\mathrm{Z}$

Density (calculated)

Absorption coefficient

$\mathrm{F}(000)$

Crystal size sp5010

C17 H22 O3 S2

338.47

293(2) K

1.54176 А̊

Monoclinic

$\mathrm{P} 21 / \mathrm{c}$

$\mathrm{a}=12.956(4) \AA$

$\alpha=90^{\circ}$.

$\mathrm{b}=7.608(3) \AA$

$\beta=99.89(3)^{\circ}$.

c $=17.398(7) \AA$

$\gamma=90^{\circ}$.

\section{4}

$1.331 \mathrm{Mg} / \mathrm{m}^{3}$

$2.935 \mathrm{~mm}^{-1}$

720

$0.30 \times 0.20 \times 0.10 \mathrm{~mm}^{3}$ 
Theta range for data collection

Index ranges

Reflections collected

Independent reflections

Completeness to theta $=69.95^{\circ}$

Absorption correction

Max. and min. transmission

Refinement method

Data / restraints / parameters

Goodness-of-fit on $\mathrm{F}^{2}$

Final R indices [I>2sigma(I)]

$\mathrm{R}$ indices (all data)

Extinction coefficient

Largest diff. peak and hole
3.46 to $69.95^{\circ}$.

$-15<=\mathrm{h}<=15,0<=\mathrm{k}<=9,0<=\mathrm{l}<=21$

3049

$3049[\mathrm{R}(\mathrm{int})=0.0000]$

$95.3 \%$

Empirical

0.7579 and 0.4730

Full-matrix least-squares on $\mathrm{F}^{2}$

3049 / 0 / 203

0.975

$\mathrm{R} 1=0.0844, \mathrm{wR} 2=0.2281$

$\mathrm{R} 1=0.1476, \mathrm{wR} 2=0.2697$

$0.0125(18)$

0.355 and -0.344 e. $\AA^{-3}$

Table 2. Atomic coordinates ( $\left.\mathrm{x} 10^{4}\right)$ and equivalent isotropic displacement parameters $\left(\AA^{2} \mathrm{x} 10^{3}\right)$ for sp5010. $U(\mathrm{eq})$ is defined as one third of the trace of the orthogonalized $\mathrm{U}^{\mathrm{ij}}$ tensor.

\begin{tabular}{|c|c|c|c|c|}
\hline & $\mathrm{x}$ & $\mathrm{y}$ & $\mathrm{z}$ & $\mathrm{U}(\mathrm{eq})$ \\
\hline$C(1)$ & $9058(5)$ & $3343(9)$ & $7918(4)$ & $108(2)$ \\
\hline $\mathrm{C}(2)$ & $8025(4)$ & 2888(8) & 8143(3) & $95(2)$ \\
\hline C(3) & 7598(5) & 1193(8) & 7801(4) & $109(2)$ \\
\hline C(5) & $6699(4)$ & $3813(7)$ & 8974(3) & $87(1)$ \\
\hline$C(10)$ & $6582(4)$ & 4685(7) & $9621(4)$ & $98(2)$ \\
\hline $\mathrm{C}(11)$ & $7367(5)$ & $5855(8)$ & $10065(4)$ & $104(2)$ \\
\hline $\mathrm{C}(12)$ & $8047(6)$ & 4839(9) & $10732(4)$ & $120(2)$ \\
\hline C(4) & $7671(4)$ & 3953(7) & $8659(3)$ & $88(1)$ \\
\hline$C(6)$ & $5808(4)$ & $2630(7)$ & $8601(3)$ & $91(2)$ \\
\hline C(7) & $4738(5)$ & $-508(8)$ & $8638(4)$ & $110(2)$ \\
\hline C(8) & $3694(5)$ & $469(8)$ & $8460(4)$ & $108(2)$ \\
\hline $\mathrm{C}(9)$ & $3733(5)$ & 2106(8) & $7985(4)$ & $109(2)$ \\
\hline$C(13)$ & $8016(4)$ & $6777(7)$ & $9505(3)$ & $93(2)$ \\
\hline $\mathrm{C}(14)$ & $8872(5)$ & 7863(7) & $9962(4)$ & $98(2)$ \\
\hline$C(15)$ & $8581(5)$ & $9646(8)$ & $10220(5)$ & $134(3)$ \\
\hline$C(16)$ & $8439(4)$ & 5389(7) & $9005(3)$ & $89(2)$ \\
\hline$C(17)$ & $8902(5)$ & $6263(10)$ & $8358(4)$ & $109(2)$ \\
\hline $\mathrm{O}(1)$ & 9204(3) & $5214(6)$ & 7828(3) & $115(1)$ \\
\hline $\mathrm{O}(2)$ & $9756(3)$ & $7308(5)$ & 10141(3) & $117(1)$ \\
\hline
\end{tabular}




\begin{tabular}{llrll}
$\mathrm{O}(3)$ & $9032(4)$ & $7842(6)$ & $8323(3)$ & $132(2)$ \\
$\mathrm{S}(1)$ & $5782(1)$ & $713(2)$ & $9220(1)$ & $102(1)$ \\
$\mathrm{S}(2)$ & $4576(1)$ & $3829(2)$ & $8463(1)$ & $100(1)$ \\
\hline
\end{tabular}

Table 3. Bond lengths $[\AA]$ and angles $\left[{ }^{\circ}\right]$ for sp5010.

\begin{tabular}{|c|c|}
\hline $\mathrm{C}(1)-\mathrm{O}(1)$ & $1.448(7)$ \\
\hline$C(1)-C(2)$ & $1.498(8)$ \\
\hline$C(2)-C(4)$ & $1.348(7)$ \\
\hline C(2)-C(3) & $1.487(8)$ \\
\hline C(5)-C(10) & $1.339(7)$ \\
\hline$C(5)-C(4)$ & $1.460(8)$ \\
\hline$C(5)-C(6)$ & $1.519(7)$ \\
\hline$C(10)-C(11)$ & $1.468(7)$ \\
\hline $\mathrm{C}(11)-\mathrm{C}(12)$ & $1.539(9)$ \\
\hline C(11)-C(13) & $1.559(8)$ \\
\hline C(4)-C(16) & $1.529(7)$ \\
\hline$C(6)-S(1)$ & $1.817(5)$ \\
\hline$C(6)-S(2)$ & $1.819(5)$ \\
\hline C(7)-C(8) & $1.528(8)$ \\
\hline$C(7)-S(1)$ & $1.801(6)$ \\
\hline $\mathrm{C}(8)-\mathrm{C}(9)$ & $1.500(8)$ \\
\hline$C(9)-S(2)$ & $1.814(6)$ \\
\hline C(13)-C(14) & $1.498(7)$ \\
\hline$C(13)-C(16)$ & $1.529(7)$ \\
\hline $\mathrm{C}(14)-\mathrm{O}(2)$ & $1.210(6)$ \\
\hline C(14)-C(15) & $1.497(8)$ \\
\hline C(16)-C(17) & $1.517(9)$ \\
\hline $\mathrm{C}(17)-\mathrm{O}(3)$ & $1.216(8)$ \\
\hline $\mathrm{C}(17)-\mathrm{O}(1)$ & $1.328(8)$ \\
\hline $\mathrm{O}(1)-\mathrm{C}(1)-\mathrm{C}(2)$ & $113.2(5)$ \\
\hline C(4)-C(2)-C(3) & 129.9(6) \\
\hline $\mathrm{C}(4)-\mathrm{C}(2)-\mathrm{C}(1)$ & 117.2(5) \\
\hline $\mathrm{C}(3)-\mathrm{C}(2)-\mathrm{C}(1)$ & $112.6(5)$ \\
\hline$C(10)-C(5)-C(4)$ & $120.6(5)$ \\
\hline$C(10)-C(5)-C(6)$ & $117.7(5)$ \\
\hline $\mathrm{C}(4)-\mathrm{C}(5)-\mathrm{C}(6)$ & 121.6(5) \\
\hline $\mathrm{C}(5)-\mathrm{C}(10)-\mathrm{C}(11)$ & 125.1(5) \\
\hline $\mathrm{C}(10)-\mathrm{C}(11)-\mathrm{C}(12)$ & $110.3(5)$ \\
\hline
\end{tabular}




\begin{tabular}{|c|c|}
\hline $\mathrm{C}(10)-\mathrm{C}(11)-\mathrm{C}(13)$ & 110.1(5) \\
\hline C(12)-C(11)-C(13) & $113.4(5)$ \\
\hline$C(2)-C(4)-C(5)$ & $128.3(5)$ \\
\hline$C(2)-C(4)-C(16)$ & $114.7(5)$ \\
\hline$C(5)-C(4)-C(16)$ & $116.7(5)$ \\
\hline$C(5)-C(6)-S(1)$ & $108.2(4)$ \\
\hline$C(5)-C(6)-S(2)$ & $110.2(4)$ \\
\hline$S(1)-C(6)-S(2)$ & $112.0(3)$ \\
\hline$C(8)-C(7)-S(1)$ & $114.6(4)$ \\
\hline C(9)-C(8)-C(7) & $113.8(5)$ \\
\hline $\mathrm{C}(8)-\mathrm{C}(9)-\mathrm{S}(2)$ & $114.9(4)$ \\
\hline C(14)-C(13)-C(16) & $112.2(5)$ \\
\hline C(14)-C(13)-C(11) & $110.3(5)$ \\
\hline $\mathrm{C}(16)-\mathrm{C}(13)-\mathrm{C}(11)$ & $109.3(4)$ \\
\hline $\mathrm{O}(2)-\mathrm{C}(14)-\mathrm{C}(15)$ & $121.0(5)$ \\
\hline $\mathrm{O}(2)-\mathrm{C}(14)-\mathrm{C}(13)$ & $121.7(5)$ \\
\hline C(15)-C(14)-C(13) & $117.2(5)$ \\
\hline $\mathrm{C}(17)-\mathrm{C}(16)-\mathrm{C}(13)$ & $110.2(5)$ \\
\hline $\mathrm{C}(17)-\mathrm{C}(16)-\mathrm{C}(4)$ & $109.5(5)$ \\
\hline $\mathrm{C}(13)-\mathrm{C}(16)-\mathrm{C}(4)$ & $116.4(4)$ \\
\hline $\mathrm{O}(3)-\mathrm{C}(17)-\mathrm{O}(1)$ & $119.9(7)$ \\
\hline $\mathrm{O}(3)-\mathrm{C}(17)-\mathrm{C}(16)$ & 123.1(7) \\
\hline $\mathrm{O}(1)-\mathrm{C}(17)-\mathrm{C}(16)$ & $116.9(6)$ \\
\hline $\mathrm{C}(17)-\mathrm{O}(1)-\mathrm{C}(1)$ & $117.2(5)$ \\
\hline$C(7)-S(1)-C(6)$ & $99.8(3)$ \\
\hline $\mathrm{C}(9)-\mathrm{S}(2)-\mathrm{C}(6)$ & 98.2(3) \\
\hline
\end{tabular}

Symmetry transformations used to generate equivalent atoms:

Table 4. Anisotropic displacement parameters $\left(\AA^{2} \mathrm{x} 10^{3}\right)$ for sp5010. The anisotropic displacement factor exponent takes the form: $-2 \pi^{2}\left[h^{2} a^{* 2} U^{11}+\ldots+2 h k a^{*} b^{*} U^{12}\right]$

\begin{tabular}{lcccccc}
\hline & $\mathrm{U}^{11}$ & $\mathrm{U}^{22}$ & $\mathrm{U}^{33}$ & $\mathrm{U}^{23}$ & $\mathrm{U}^{13}$ & $\mathrm{U}^{12}$ \\
\hline $\mathrm{C}(1)$ & $99(4)$ & $102(4)$ & $120(5)$ & $-13(4)$ & $11(3)$ & $2(3)$ \\
$\mathrm{C}(2)$ & $83(3)$ & $90(4)$ & $108(4)$ & $-6(3)$ & $6(3)$ & $7(3)$ \\
$\mathrm{C}(3)$ & $106(4)$ & $92(4)$ & $125(5)$ & $-15(3)$ & $12(4)$ & $6(3)$ \\
$\mathrm{C}(5)$ & $84(3)$ & $79(3)$ & $91(3)$ & $3(3)$ & $0(3)$ & $-1(3)$ \\
$\mathrm{C}(10)$ & $88(4)$ & $94(4)$ & $111(4)$ & $-20(3)$ & $17(3)$ & $-27(3)$ \\
$\mathrm{C}(11)$ & $94(4)$ & $93(4)$ & $126(5)$ & $-19(3)$ & $23(3)$ & $-13(3)$ \\
& & & & & & \\
\end{tabular}




\begin{tabular}{lcccccc}
$\mathrm{C}(12)$ & $128(5)$ & $123(5)$ & $103(4)$ & $0(4)$ & $6(4)$ & $-24(4)$ \\
$\mathrm{C}(4)$ & $81(3)$ & $71(3)$ & $106(4)$ & $1(3)$ & $-2(3)$ & $0(3)$ \\
$\mathrm{C}(6)$ & $90(3)$ & $73(3)$ & $107(4)$ & $3(3)$ & $7(3)$ & $0(3)$ \\
$\mathrm{C}(7)$ & $124(5)$ & $81(4)$ & $120(5)$ & $-11(3)$ & $11(4)$ & $-16(3)$ \\
$\mathrm{C}(8)$ & $101(4)$ & $93(4)$ & $127(5)$ & $-18(4)$ & $11(4)$ & $-16(3)$ \\
$\mathrm{C}(9)$ & $96(4)$ & $103(4)$ & $122(5)$ & $-9(4)$ & $-5(3)$ & $-4(3)$ \\
$\mathrm{C}(13)$ & $88(3)$ & $70(3)$ & $115(4)$ & $-3(3)$ & $5(3)$ & $0(3)$ \\
$\mathrm{C}(14)$ & $85(3)$ & $69(3)$ & $135(5)$ & $-1(3)$ & $12(3)$ & $-12(3)$ \\
$\mathrm{C}(15)$ & $108(5)$ & $81(4)$ & $208(8)$ & $-35(4)$ & $17(5)$ & $-5(3)$ \\
$\mathrm{C}(16)$ & $84(3)$ & $71(3)$ & $110(4)$ & $6(3)$ & $8(3)$ & $9(3)$ \\
$\mathrm{C}(17)$ & $92(4)$ & $103(5)$ & $134(5)$ & $14(4)$ & $25(4)$ & $15(4)$ \\
$\mathrm{O}(1)$ & $112(3)$ & $110(3)$ & $128(3)$ & $-1(3)$ & $36(3)$ & $-5(3)$ \\
$\mathrm{O}(2)$ & $94(3)$ & $85(2)$ & $159(4)$ & $-13(2)$ & $-9(3)$ & $3(2)$ \\
$\mathrm{O}(3)$ & $141(4)$ & $89(3)$ & $175(4)$ & $30(3)$ & $49(3)$ & $8(3)$ \\
$\mathrm{S}(1)$ & $108(1)$ & $82(1)$ & $111(1)$ & $7(1)$ & $3(1)$ & $-6(1)$ \\
$\mathrm{S}(2)$ & $87(1)$ & $81(1)$ & $128(1)$ & $-2(1)$ & $4(1)$ & $-1(1)$ \\
\hline
\end{tabular}

Table 5. Hydrogen coordinates ( $\left.\times 10^{4}\right)$ and isotropic displacement parameters $\left(\AA^{2} \times 10^{3}\right)$ for sp5010.

\begin{tabular}{|c|c|c|c|c|}
\hline & $x$ & $\mathrm{y}$ & $\mathrm{z}$ & $\mathrm{U}(\mathrm{eq})$ \\
\hline $\mathrm{H}(1 \mathrm{~A})$ & 9616 & 2898 & 8313 & 129 \\
\hline $\mathrm{H}(1 \mathrm{~B})$ & 9111 & 2761 & 7430 & 129 \\
\hline $\mathrm{H}(3 \mathrm{~A})$ & 7251 & 590 & 8169 & 163 \\
\hline $\mathrm{H}(3 \mathrm{~B})$ & 8161 & 479 & 7682 & 163 \\
\hline $\mathrm{H}(3 \mathrm{C})$ & 7107 & 1416 & 7332 & 163 \\
\hline $\mathrm{H}(10)$ & 5954 & 4538 & 9804 & 117 \\
\hline $\mathrm{H}(11)$ & 6991 & 6771 & 10302 & 124 \\
\hline $\mathrm{H}(12 \mathrm{~A})$ & 7604 & 4272 & 11046 & 180 \\
\hline $\mathrm{H}(12 \mathrm{~B})$ & 8509 & 5641 & 11049 & 180 \\
\hline $\mathrm{H}(12 \mathrm{C})$ & 8453 & 3969 & 10517 & 180 \\
\hline $\mathrm{H}(6)$ & 5945 & 2245 & 8090 & 109 \\
\hline $\mathrm{H}(7 \mathrm{~A})$ & 4953 & -822 & 8149 & 132 \\
\hline $\mathrm{H}(7 \mathrm{~B})$ & 4634 & -1591 & 8909 & 132 \\
\hline $\mathrm{H}(8 \mathrm{~A})$ & 3169 & -315 & 8181 & 130 \\
\hline $\mathrm{H}(8 \mathrm{~B})$ & 3476 & 781 & 8948 & 130 \\
\hline $\mathrm{H}(9 \mathrm{~A})$ & 3028 & 2569 & 7847 & 131 \\
\hline
\end{tabular}




\begin{tabular}{lrrrr}
$\mathrm{H}(9 \mathrm{~B})$ & 3971 & 1794 & 7504 & 131 \\
$\mathrm{H}(13)$ & 7548 & 7560 & 9159 & 111 \\
$\mathrm{H}(15 \mathrm{~A})$ & 8925 & 9852 & 10746 & 201 \\
$\mathrm{H}(15 \mathrm{~B})$ & 7836 & 9706 & 10195 & 201 \\
$\mathrm{H}(15 \mathrm{C})$ & 8795 & 10524 & 9883 & 201 \\
$\mathrm{H}(16)$ & 9018 & 4797 & 9343 & 107 \\
\hline
\end{tabular}

Table 6. Torsion angles $\left[^{\circ}\right]$ for sp5010.

\begin{tabular}{|c|c|}
\hline $\mathrm{O}(1)-\mathrm{C}(1)-\mathrm{C}(2)-\mathrm{C}(4)$ & $-39.1(8)$ \\
\hline $\mathrm{O}(1)-\mathrm{C}(1)-\mathrm{C}(2)-\mathrm{C}(3)$ & $146.2(5)$ \\
\hline$C(4)-C(5)-C(10)-C(11)$ & $1.1(9)$ \\
\hline C(6)-C(5)-C(10)-C(11) & $178.8(5)$ \\
\hline C(5)-C(10)-C(11)-C(12) & $-93.9(7)$ \\
\hline C(5)-C(10)-C(11)-C(13) & $31.9(8)$ \\
\hline $\mathrm{C}(3)-\mathrm{C}(2)-\mathrm{C}(4)-\mathrm{C}(5)$ & $-6.4(10)$ \\
\hline $\mathrm{C}(1)-\mathrm{C}(2)-\mathrm{C}(4)-\mathrm{C}(5)$ & $180.0(5)$ \\
\hline C(3)-C(2)-C(4)-C(16) & $167.8(5)$ \\
\hline $\mathrm{C}(1)-\mathrm{C}(2)-\mathrm{C}(4)-\mathrm{C}(16)$ & $-5.8(7)$ \\
\hline$C(10)-C(5)-C(4)-C(2)$ & $162.6(6)$ \\
\hline$C(6)-C(5)-C(4)-C(2)$ & $-15.0(8)$ \\
\hline C(10)-C(5)-C(4)-C(16) & $-11.5(7)$ \\
\hline $\mathrm{C}(6)-\mathrm{C}(5)-\mathrm{C}(4)-\mathrm{C}(16)$ & $170.9(4)$ \\
\hline$C(10)-C(5)-C(6)-S(1)$ & $-71.8(6)$ \\
\hline$C(4)-C(5)-C(6)-S(1)$ & $105.9(5)$ \\
\hline$C(10)-C(5)-C(6)-S(2)$ & $51.0(6)$ \\
\hline $\mathrm{C}(4)-\mathrm{C}(5)-\mathrm{C}(6)-\mathrm{S}(2)$ & $-131.3(5)$ \\
\hline$S(1)-C(7)-C(8)-C(9)$ & $62.9(7)$ \\
\hline $\mathrm{C}(7)-\mathrm{C}(8)-\mathrm{C}(9)-\mathrm{S}(2)$ & $-64.8(6)$ \\
\hline $\mathrm{C}(10)-\mathrm{C}(11)-\mathrm{C}(13)-\mathrm{C}(14)$ & $-175.6(5)$ \\
\hline $\mathrm{C}(12)-\mathrm{C}(11)-\mathrm{C}(13)-\mathrm{C}(14)$ & $-51.5(6)$ \\
\hline $\mathrm{C}(10)-\mathrm{C}(11)-\mathrm{C}(13)-\mathrm{C}(16)$ & $-51.8(6)$ \\
\hline $\mathrm{C}(12)-\mathrm{C}(11)-\mathrm{C}(13)-\mathrm{C}(16)$ & $72.3(6)$ \\
\hline $\mathrm{C}(16)-\mathrm{C}(13)-\mathrm{C}(14)-\mathrm{O}(2)$ & $-25.7(8)$ \\
\hline $\mathrm{C}(11)-\mathrm{C}(13)-\mathrm{C}(14)-\mathrm{O}(2)$ & $96.4(7)$ \\
\hline $\mathrm{C}(16)-\mathrm{C}(13)-\mathrm{C}(14)-\mathrm{C}(15)$ & $156.8(6)$ \\
\hline $\mathrm{C}(11)-\mathrm{C}(13)-\mathrm{C}(14)-\mathrm{C}(15)$ & $-81.1(7)$ \\
\hline $\mathrm{C}(14)-\mathrm{C}(13)-\mathrm{C}(16)-\mathrm{C}(17)$ & $-67.6(6)$ \\
\hline $\mathrm{C}(11)-\mathrm{C}(13)-\mathrm{C}(16)-\mathrm{C}(17)$ & $169.7(5)$ \\
\hline
\end{tabular}




$\begin{array}{lc}\mathrm{C}(14)-\mathrm{C}(13)-\mathrm{C}(16)-\mathrm{C}(4) & 166.8(4) \\ \mathrm{C}(11)-\mathrm{C}(13)-\mathrm{C}(16)-\mathrm{C}(4) & 44.2(6) \\ \mathrm{C}(2)-\mathrm{C}(4)-\mathrm{C}(16)-\mathrm{C}(17) & 46.0(6) \\ \mathrm{C}(5)-\mathrm{C}(4)-\mathrm{C}(16)-\mathrm{C}(17) & -139.1(5) \\ \mathrm{C}(2)-\mathrm{C}(4)-\mathrm{C}(16)-\mathrm{C}(13) & 171.9(5) \\ \mathrm{C}(5)-\mathrm{C}(4)-\mathrm{C}(16)-\mathrm{C}(13) & -13.2(7) \\ \mathrm{C}(13)-\mathrm{C}(16)-\mathrm{C}(17)-\mathrm{O}(3) & 9.0(8) \\ \mathrm{C}(4)-\mathrm{C}(16)-\mathrm{C}(17)-\mathrm{O}(3) & 138.3(6) \\ \mathrm{C}(13)-\mathrm{C}(16)-\mathrm{C}(17)-\mathrm{O}(1) & -173.3(5) \\ \mathrm{C}(4)-\mathrm{C}(16)-\mathrm{C}(17)-\mathrm{O}(1) & -44.0(7) \\ \mathrm{O}(3)-\mathrm{C}(17)-\mathrm{O}(1)-\mathrm{C}(1) & 178.0(6) \\ \mathrm{C}(16)-\mathrm{C}(17)-\mathrm{O}(1)-\mathrm{C}(1) & 0.2(8) \\ \mathrm{C}(2)-\mathrm{C}(1)-\mathrm{O}(1)-\mathrm{C}(17) & 42.2(8) \\ \mathrm{C}(8)-\mathrm{C}(7)-\mathrm{S}(1)-\mathrm{C}(6) & -58.0(5) \\ \mathrm{C}(5)-\mathrm{C}(6)-\mathrm{S}(1)-\mathrm{C}(7) & -176.8(4) \\ \mathrm{S}(2)-\mathrm{C}(6)-\mathrm{S}(1)-\mathrm{C}(7) & 61.5(4) \\ \mathrm{C}(8)-\mathrm{C}(9)-\mathrm{S}(2)-\mathrm{C}(6) & 60.8(5) \\ \mathrm{C}(5)-\mathrm{C}(6)-\mathrm{S}(2)-\mathrm{C}(9) & 177.6(4) \\ \mathrm{S}(1)-\mathrm{C}(6)-\mathrm{S}(2)-\mathrm{C}(9) & -61.9(4) \\ & \end{array}$

Symmetry transformations used to generate equivalent atoms: 


\section{Lactone 6a (CCDC 600915)}

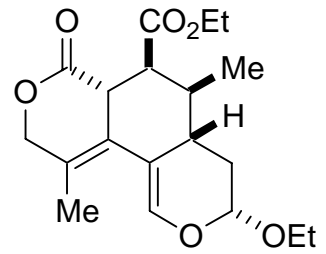

Crystals of $\mathrm{X}$ were grown by cooling of a saturated solution using ethanol at room temperature. Single crystals were coated with Paratone-N oil, mounted using a glass fibre and frozen in the cold nitrogen stream of the goniometer. A hemisphere of data was collected on a Bruker AXS P4/SMART 1000 diffractometer using $\omega$ and $\theta$ scans with a scan width of $0.3^{\circ}$ and $30 \mathrm{~s}$ exposure times. The detector distance was $5 \mathrm{~cm}$. The data were reduced (SAINT) ${ }^{1}$ and corrected for absorption (SADABS). ${ }^{2}$ The structure was solved by direct methods and refined by full-matrix least squares on $\mathrm{F}^{2}$ (SHELXTL). ${ }^{3}$ All non-hydrogen atoms were refined anisotropically. Hydrogen atoms were included in calculated positions and refined using a riding model.

(1) SAINT 6.02, 1997-1999, Bruker AXS, Inc., Madison, Wisconsin, USA.

(2) SADABS George Sheldrick, 1999, Bruker AXS, Inc., Madison,Wisconsin, USA.

(3) SHELXTL 5.1, George Sheldrick, 1997, Bruker AXS, Inc., Madison,Wisconsin, USA. 


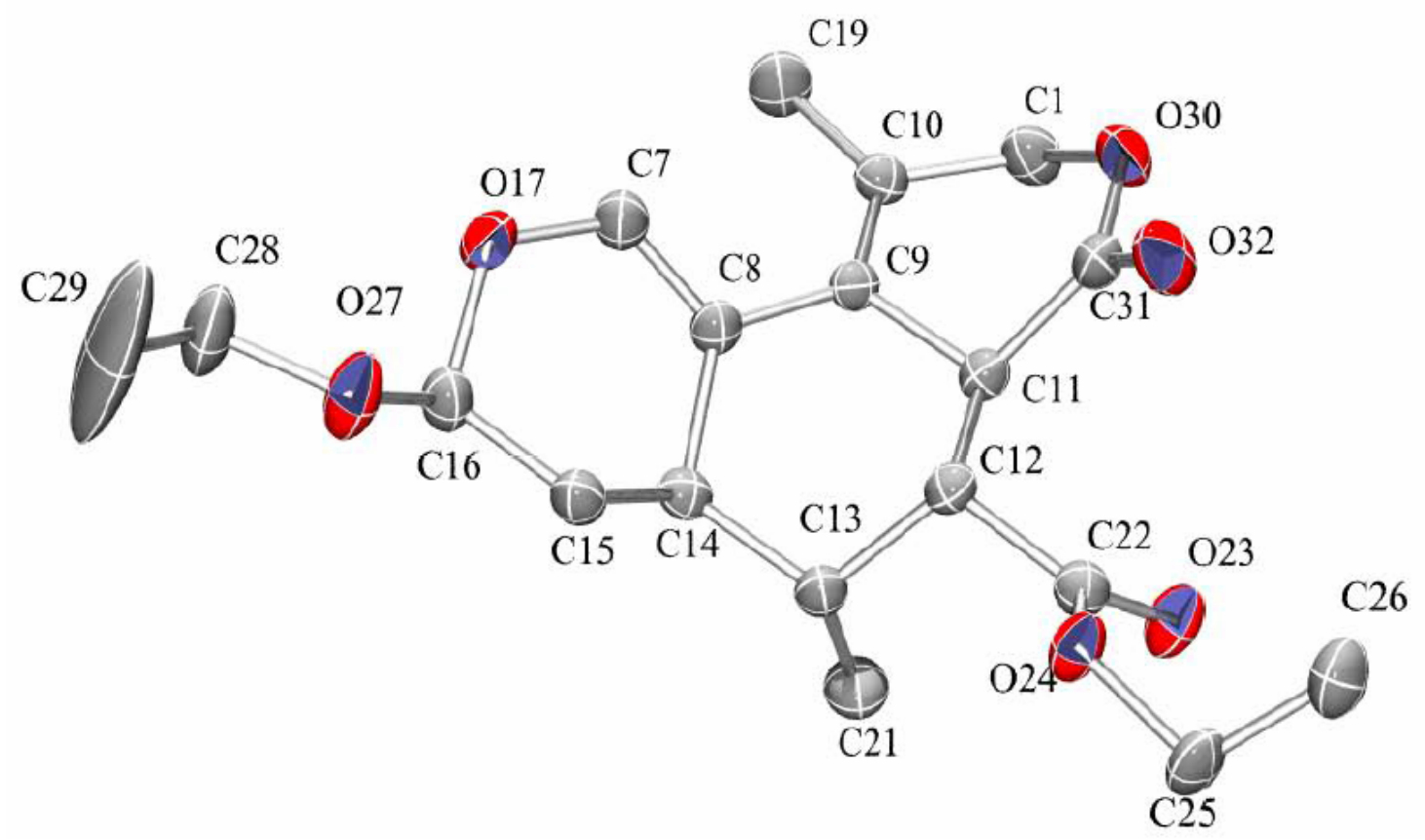

Table 1. Crystal data and structure refinement for SP2-122.

Identification code

Empirical formula

Formula weight

Temperature

Wavelength

Diffractometer used

Detector distance

Monochrometer used

Crystal size

Colour and habit

Crystal system

Space group

Unit cell dimensions

Volume

Z

Density (calculated)

Absorption coefficient

$\mathrm{F}(000)$

Theta range for data collection

Completeness to theta $=27.50^{\circ}$

Scan type
CS040271

C19 H26 O6

350.40

198(1) K

$0.71073 \AA$

Bruker AXS P4/SMART 1000

$5 \mathrm{~cm}$

Graphite

$0.50 \times 0.10 \times 0.025 \mathrm{~mm}^{3}$

Colourless, needle

Orthorhombic

P2(1)2(1)2(1)

$\begin{array}{ll}\mathrm{a}=9.3169(12) \AA & \alpha=90^{\circ} \\ \mathrm{b}=10.0042(14) \AA & \beta=90^{\circ} \\ \mathrm{c}=19.751(3) \AA & \gamma=90^{\circ}\end{array}$

1840.9(4) $\AA^{3}$

4

$1.264 \mathrm{Mg} / \mathrm{m}^{3}$

$0.093 \mathrm{~mm}^{-1}$

752

2.06 to $27.50^{\circ}$

$96.7 \%$

$\omega$ and $\phi$ 
Scan range

Exposure time

Index ranges

Standard reflections

Crystal stability

Reflections collected

Independent reflections

System used

Solution

Hydrogen atoms

Absorption correction

Min./Max. transmission ratio

Refinement method

Data / restraints / parameters

Goodness-of-fit on $\mathrm{F}^{2}$

Final R indices [I>2sigma(I)]

$\mathrm{R}$ indices (all data)

Largest/mean shift/esd

Absolute structure parameter

Largest diff. peak and hole $0.3^{\circ}$

30 s

$-10 \leq \mathrm{h} \leq 12,-12 \leq \mathrm{k} \leq 12,-25 \leq \mathrm{l} \leq 25$

50 frames at beginning and end of data collection no decay

12311

4078 [R(int) $=0.0493]$

SHELXL 5.1

Direct methods

Calculated positions, riding model

SADABS

0.870

Full-matrix least-squares on $\mathrm{F}^{2}$

4078 / 0 / 230

1.041

$\mathrm{R} 1=0.0451, \mathrm{wR} 2=0.0850$

$\mathrm{R} 1=0.0926, \mathrm{wR} 2=0.0949$

$0.000 / 0.000$

$-0.6(11)$

0.204 and -0.176 e. $\AA^{-3}$

$\mathrm{wR} 2=\left(\sum\left[\mathrm{w}\left(\mathrm{F}_{\mathrm{o}}^{2}-\mathrm{F}_{\mathrm{c}}^{2}\right)^{2}\right] / \Sigma\left[\mathrm{F}_{\mathrm{o}}^{4}\right]\right)^{1 / 2}$

$\mathrm{R} 1=\sum|| \mathrm{F}_{\mathrm{o}}|-| \mathrm{F}_{\mathrm{C}}|| / \sum\left|\mathrm{F}_{\mathrm{o}}\right|$

Weight $=1 /\left[\sigma^{2}\left(\mathrm{~F}_{\mathrm{o}}^{2}\right)+(0.0372 * \mathrm{P})^{2}+(0.0894 * \mathrm{P})\right]$

where $\mathrm{P}=\left(\max \left(\mathrm{F}_{\mathrm{o}}^{2}, 0\right)+2 * \mathrm{~F}_{\mathrm{c}}^{2}\right) / 3$

Table 2. Atomic coordinates ( $\times 10^{4}$ ) and equivalent isotropic displacement parameters $\left(\AA^{2} \times 10^{3}\right)$ for SP2-122. $U(\mathrm{eq})$ is defined as one third of the trace of the orthogonalized $\mathrm{U}^{\mathrm{ij}}$ tensor.

\begin{tabular}{lcccc}
\hline & $\mathrm{x}$ & $\mathrm{y}$ & $\mathrm{z}$ & $\mathrm{U}(\mathrm{eq})$ \\
\hline $\mathrm{C}(1)$ & $9282(2)$ & $5516(2)$ & $2411(1)$ & $33(1)$ \\
$\mathrm{C}(7)$ & $5275(2)$ & $4292(2)$ & $3499(1)$ & $30(1)$ \\
$\mathrm{C}(8)$ & $5373(2)$ & $4718(2)$ & $2864(1)$ & $24(1)$ \\
$\mathrm{C}(9)$ & $6786(2)$ & $4967(2)$ & $2556(1)$ & $24(1)$ \\
$\mathrm{C}(10)$ & $7948(2)$ & $5515(2)$ & $2840(1)$ & $26(1)$ \\
$\mathrm{C}(11)$ & $6976(2)$ & $4492(2)$ & $1824(1)$ & $24(1)$ \\
$\mathrm{C}(12)$ & $5681(2)$ & $3733(2)$ & $1557(1)$ & $24(1)$ \\
$\mathrm{C}(13)$ & $4285(2)$ & $4549(2)$ & $1669(1)$ & $25(1)$
\end{tabular}




\begin{tabular}{lrrrr}
$\mathrm{C}(14)$ & $4022(2)$ & $4798(2)$ & $2431(1)$ & $24(1)$ \\
$\mathrm{C}(15)$ & $2943(2)$ & $3822(2)$ & $2727(1)$ & $29(1)$ \\
$\mathrm{C}(16)$ & $2717(2)$ & $4063(2)$ & $3468(1)$ & $30(1)$ \\
$\mathrm{O}(17)$ & $4053(2)$ & $3953(2)$ & $3839(1)$ & $35(1)$ \\
$\mathrm{C}(19)$ & $8072(2)$ & $6150(2)$ & $3526(1)$ & $37(1)$ \\
$\mathrm{C}(21)$ & $4265(3)$ & $5850(2)$ & $1266(1)$ & $39(1)$ \\
$\mathrm{C}(22)$ & $5847(2)$ & $3410(2)$ & $815(1)$ & $27(1)$ \\
$\mathrm{O}(23)$ & $6525(2)$ & $4068(2)$ & $414(1)$ & $37(1)$ \\
$\mathrm{O}(24)$ & $5070(2)$ & $2338(2)$ & $649(1)$ & $32(1)$ \\
$\mathrm{C}(25)$ & $5023(2)$ & $1971(3)$ & $-67(1)$ & $34(1)$ \\
$\mathrm{C}(26)$ & $6304(3)$ & $1161(3)$ & $-255(1)$ & $40(1)$ \\
$\mathrm{O}(27)$ & $1813(2)$ & $3084(2)$ & $3717(1)$ & $44(1)$ \\
$\mathrm{C}(28)$ & $1274(3)$ & $3306(3)$ & $4387(1)$ & $49(1)$ \\
$\mathrm{C}(29)$ & $204(5)$ & $2240(5)$ & $4537(2)$ & $139(2)$ \\
$\mathrm{O}(30)$ & $9495(2)$ & $4246(2)$ & $2062(1)$ & $37(1)$ \\
$\mathrm{C}(31)$ & $8323(2)$ & $3666(3)$ & $1794(1)$ & $31(1)$ \\
$\mathrm{O}(32)$ & $8411(2)$ & $2553(2)$ & $1565(1)$ & $42(1)$ \\
& & & & \\
\hline
\end{tabular}

Table 3. Bond lengths $[\AA]$ and angles $\left[{ }^{\circ}\right]$ for SP2-122.

\begin{tabular}{ll}
\hline $\mathrm{C}(1)-\mathrm{O}(30)$ & $1.459(3)$ \\
$\mathrm{C}(1)-\mathrm{C}(10)$ & $1.504(3)$ \\
$\mathrm{C}(1)-\mathrm{H}(1 \mathrm{~A})$ & 0.9900 \\
$\mathrm{C}(1)-\mathrm{H}(1 \mathrm{~B})$ & 0.9900 \\
$\mathrm{C}(7)-\mathrm{C}(8)$ & $1.328(3)$ \\
$\mathrm{C}(7)-\mathrm{O}(17)$ & $1.365(2)$ \\
$\mathrm{C}(7)-\mathrm{H}(7)$ & 0.9500 \\
$\mathrm{C}(8)-\mathrm{C}(9)$ & $1.471(3)$ \\
$\mathrm{C}(8)-\mathrm{C}(14)$ & $1.524(3)$ \\
$\mathrm{C}(9)-\mathrm{C}(10)$ & $1.337(3)$ \\
$\mathrm{C}(9)-\mathrm{C}(11)$ & $1.533(3)$ \\
$\mathrm{C}(10)-\mathrm{C}(19)$ & $1.503(3)$ \\
$\mathrm{C}(11)-\mathrm{C}(31)$ & $1.504(3)$ \\
$\mathrm{C}(11)-\mathrm{C}(12)$ & $1.519(3)$ \\
$\mathrm{C}(11)-\mathrm{H}(11)$ & 1.0000 \\
$\mathrm{C}(12)-\mathrm{C}(22)$ & $1.510(3)$ \\
$\mathrm{C}(12)-\mathrm{C}(13)$ & $1.551(3)$ \\
$\mathrm{C}(12)-\mathrm{H}(12)$ & 1.0000 \\
$\mathrm{C}(13)-\mathrm{C}(21)$ & $1.525(3)$ \\
$\mathrm{C}(13)-\mathrm{C}(14)$ & $1.547(3)$ \\
\end{tabular}




\begin{tabular}{|c|c|}
\hline C(13)-H(13) & 1.0000 \\
\hline $\mathrm{C}(14)-\mathrm{C}(15)$ & $1.518(3)$ \\
\hline $\mathrm{C}(14)-\mathrm{H}(14)$ & 1.0000 \\
\hline $\mathrm{C}(15)-\mathrm{C}(16)$ & 1.499(3) \\
\hline C(15)-H(15A) & 0.9900 \\
\hline C(15)-H(15B) & 0.9900 \\
\hline $\mathrm{C}(16)-\mathrm{O}(27)$ & $1.382(3)$ \\
\hline $\mathrm{C}(16)-\mathrm{O}(17)$ & $1.448(2)$ \\
\hline $\mathrm{C}(16)-\mathrm{H}(16)$ & 1.0000 \\
\hline C(19)-H(19A) & 0.9800 \\
\hline C(19)-H(19B) & 0.9800 \\
\hline C(19)-H(19C) & 0.9800 \\
\hline $\mathrm{C}(21)-\mathrm{H}(21 \mathrm{~A})$ & 0.9800 \\
\hline $\mathrm{C}(21)-\mathrm{H}(21 \mathrm{~B})$ & 0.9800 \\
\hline C(21)-H(21C) & 0.9800 \\
\hline $\mathrm{C}(22)-\mathrm{O}(23)$ & $1.208(3)$ \\
\hline $\mathrm{C}(22)-\mathrm{O}(24)$ & $1.335(3)$ \\
\hline $\mathrm{O}(24)-\mathrm{C}(25)$ & $1.461(2)$ \\
\hline C(25)-C(26) & 1.489(3) \\
\hline $\mathrm{C}(25)-\mathrm{H}(25 \mathrm{~A})$ & 0.9900 \\
\hline $\mathrm{C}(25)-\mathrm{H}(25 \mathrm{~B})$ & 0.9900 \\
\hline $\mathrm{C}(26)-\mathrm{H}(26 \mathrm{~A})$ & 0.9800 \\
\hline C(26)-H(26B) & 0.9800 \\
\hline C(26)-H(26C) & 0.9800 \\
\hline $\mathrm{O}(27)-\mathrm{C}(28)$ & 1.433(3) \\
\hline C(28)-C(29) & $1.490(4)$ \\
\hline $\mathrm{C}(28)-\mathrm{H}(28 \mathrm{~A})$ & 0.9900 \\
\hline $\mathrm{C}(28)-\mathrm{H}(28 \mathrm{~B})$ & 0.9900 \\
\hline C(29)-H(29A) & 0.9800 \\
\hline C(29)-H(29B) & 0.9800 \\
\hline C(29)-H(29C) & 0.9800 \\
\hline $\mathrm{O}(30)-\mathrm{C}(31)$ & $1.345(3)$ \\
\hline $\mathrm{C}(31)-\mathrm{O}(32)$ & $1.205(3)$ \\
\hline $\mathrm{O}(30)-\mathrm{C}(1)-\mathrm{C}(10)$ & $112.20(19)$ \\
\hline $\mathrm{O}(30)-\mathrm{C}(1)-\mathrm{H}(1 \mathrm{~A})$ & 109.2 \\
\hline $\mathrm{C}(10)-\mathrm{C}(1)-\mathrm{H}(1 \mathrm{~A})$ & 109.2 \\
\hline $\mathrm{O}(30)-\mathrm{C}(1)-\mathrm{H}(1 \mathrm{~B})$ & 109.2 \\
\hline $\mathrm{C}(10)-\mathrm{C}(1)-\mathrm{H}(1 \mathrm{~B})$ & 109.2 \\
\hline $\mathrm{H}(1 \mathrm{~A})-\mathrm{C}(1)-\mathrm{H}(1 \mathrm{~B})$ & 107.9 \\
\hline $\mathrm{C}(8)-\mathrm{C}(7)-\mathrm{O}(17)$ & 127.04(19) \\
\hline $\mathrm{C}(8)-\mathrm{C}(7)-\mathrm{H}(7)$ & 116.5 \\
\hline
\end{tabular}




\begin{tabular}{|c|c|}
\hline $\mathrm{O}(17)-\mathrm{C}(7)-\mathrm{H}(7)$ & 116.5 \\
\hline $\mathrm{C}(7)-\mathrm{C}(8)-\mathrm{C}(9)$ & 120.47(19) \\
\hline$C(7)-C(8)-C(14)$ & 119.32(19) \\
\hline C(9)-C(8)-C(14) & 119.86(19) \\
\hline C(10)-C(9)-C(8) & $128.4(2)$ \\
\hline C(10)-C(9)-C(11) & $115.40(18)$ \\
\hline $\mathrm{C}(8)-\mathrm{C}(9)-\mathrm{C}(11)$ & 116.18(17) \\
\hline C(9)-C(10)-C(19) & 127.89(19) \\
\hline $\mathrm{C}(9)-\mathrm{C}(10)-\mathrm{C}(1)$ & 115.68(19) \\
\hline$C(19)-C(10)-C(1)$ & $116.42(18)$ \\
\hline $\mathrm{C}(31)-\mathrm{C}(11)-\mathrm{C}(12)$ & $112.02(18)$ \\
\hline $\mathrm{C}(31)-\mathrm{C}(11)-\mathrm{C}(9)$ & 107.65(17) \\
\hline C(12)-C(11)-C(9) & 112.93(17) \\
\hline $\mathrm{C}(31)-\mathrm{C}(11)-\mathrm{H}(11)$ & 108.0 \\
\hline $\mathrm{C}(12)-\mathrm{C}(11)-\mathrm{H}(11)$ & 108.0 \\
\hline $\mathrm{C}(9)-\mathrm{C}(11)-\mathrm{H}(11)$ & 108.0 \\
\hline $\mathrm{C}(22)-\mathrm{C}(12)-\mathrm{C}(11)$ & $111.21(17)$ \\
\hline C(22)-C(12)-C(13) & 109.62(17) \\
\hline $\mathrm{C}(11)-\mathrm{C}(12)-\mathrm{C}(13)$ & $110.75(17)$ \\
\hline $\mathrm{C}(22)-\mathrm{C}(12)-\mathrm{H}(12)$ & 108.4 \\
\hline $\mathrm{C}(11)-\mathrm{C}(12)-\mathrm{H}(12)$ & 108.4 \\
\hline $\mathrm{C}(13)-\mathrm{C}(12)-\mathrm{H}(12)$ & 108.4 \\
\hline$C(21)-C(13)-C(14)$ & $111.60(18)$ \\
\hline $\mathrm{C}(21)-\mathrm{C}(13)-\mathrm{C}(12)$ & $112.64(17)$ \\
\hline C(14)-C(13)-C(12) & $110.82(17)$ \\
\hline $\mathrm{C}(21)-\mathrm{C}(13)-\mathrm{H}(13)$ & 107.2 \\
\hline $\mathrm{C}(14)-\mathrm{C}(13)-\mathrm{H}(13)$ & 107.2 \\
\hline C(12)-C(13)-H(13) & 107.2 \\
\hline C(15)-C(14)-C(8) & 107.31(18) \\
\hline C(15)-C(14)-C(13) & $112.02(18)$ \\
\hline C(8)-C(14)-C(13) & $114.04(17)$ \\
\hline $\mathrm{C}(15)-\mathrm{C}(14)-\mathrm{H}(14)$ & 107.7 \\
\hline C(8)-C(14)-H(14) & 107.7 \\
\hline $\mathrm{C}(13)-\mathrm{C}(14)-\mathrm{H}(14)$ & 107.7 \\
\hline C(16)-C(15)-C(14) & $111.40(18)$ \\
\hline $\mathrm{C}(16)-\mathrm{C}(15)-\mathrm{H}(15 \mathrm{~A})$ & 109.3 \\
\hline $\mathrm{C}(14)-\mathrm{C}(15)-\mathrm{H}(15 \mathrm{~A})$ & 109.3 \\
\hline C(16)-C(15)-H(15B) & 109.3 \\
\hline C(14)-C(15)-H(15B) & 109.3 \\
\hline $\mathrm{H}(15 \mathrm{~A})-\mathrm{C}(15)-\mathrm{H}(15 \mathrm{~B})$ & 108.0 \\
\hline $\mathrm{O}(27)-\mathrm{C}(16)-\mathrm{O}(17)$ & 106.91(18) \\
\hline $\mathrm{O}(27)-\mathrm{C}(16)-\mathrm{C}(15)$ & $108.56(18)$ \\
\hline
\end{tabular}




\begin{tabular}{|c|c|}
\hline $\mathrm{O}(17)-\mathrm{C}(16)-\mathrm{C}(15)$ & $111.20(17)$ \\
\hline $\mathrm{O}(27)-\mathrm{C}(16)-\mathrm{H}(16)$ & 110.0 \\
\hline $\mathrm{O}(17)-\mathrm{C}(16)-\mathrm{H}(16)$ & 110.0 \\
\hline $\mathrm{C}(15)-\mathrm{C}(16)-\mathrm{H}(16)$ & 110.0 \\
\hline C(7)-O(17)-C(16) & $116.68(16)$ \\
\hline $\mathrm{C}(10)-\mathrm{C}(19)-\mathrm{H}(19 \mathrm{~A})$ & 109.5 \\
\hline C(10)-C(19)-H(19B) & 109.5 \\
\hline H(19A)-C(19)-H(19B) & 109.5 \\
\hline C(10)-C(19)-H(19C) & 109.5 \\
\hline H(19A)-C(19)-H(19C) & 109.5 \\
\hline H(19B)-C(19)-H(19C) & 109.5 \\
\hline $\mathrm{C}(13)-\mathrm{C}(21)-\mathrm{H}(21 \mathrm{~A})$ & 109.5 \\
\hline C(13)-C(21)-H(21B) & 109.5 \\
\hline $\mathrm{H}(21 \mathrm{~A})-\mathrm{C}(21)-\mathrm{H}(21 \mathrm{~B})$ & 109.5 \\
\hline $\mathrm{C}(13)-\mathrm{C}(21)-\mathrm{H}(21 \mathrm{C})$ & 109.5 \\
\hline $\mathrm{H}(21 \mathrm{~A})-\mathrm{C}(21)-\mathrm{H}(21 \mathrm{C})$ & 109.5 \\
\hline $\mathrm{H}(21 \mathrm{~B})-\mathrm{C}(21)-\mathrm{H}(21 \mathrm{C})$ & 109.5 \\
\hline $\mathrm{O}(23)-\mathrm{C}(22)-\mathrm{O}(24)$ & $124.0(2)$ \\
\hline $\mathrm{O}(23)-\mathrm{C}(22)-\mathrm{C}(12)$ & $125.0(2)$ \\
\hline $\mathrm{O}(24)-\mathrm{C}(22)-\mathrm{C}(12)$ & $110.80(18)$ \\
\hline $\mathrm{C}(22)-\mathrm{O}(24)-\mathrm{C}(25)$ & $117.13(17)$ \\
\hline $\mathrm{O}(24)-\mathrm{C}(25)-\mathrm{C}(26)$ & $110.76(19)$ \\
\hline $\mathrm{O}(24)-\mathrm{C}(25)-\mathrm{H}(25 \mathrm{~A})$ & 109.5 \\
\hline $\mathrm{C}(26)-\mathrm{C}(25)-\mathrm{H}(25 \mathrm{~A})$ & 109.5 \\
\hline $\mathrm{O}(24)-\mathrm{C}(25)-\mathrm{H}(25 \mathrm{~B})$ & 109.5 \\
\hline $\mathrm{C}(26)-\mathrm{C}(25)-\mathrm{H}(25 \mathrm{~B})$ & 109.5 \\
\hline $\mathrm{H}(25 \mathrm{~A})-\mathrm{C}(25)-\mathrm{H}(25 \mathrm{~B})$ & 108.1 \\
\hline $\mathrm{C}(25)-\mathrm{C}(26)-\mathrm{H}(26 \mathrm{~A})$ & 109.5 \\
\hline C(25)-C(26)-H(26B) & 109.5 \\
\hline $\mathrm{H}(26 \mathrm{~A})-\mathrm{C}(26)-\mathrm{H}(26 \mathrm{~B})$ & 109.5 \\
\hline $\mathrm{C}(25)-\mathrm{C}(26)-\mathrm{H}(26 \mathrm{C})$ & 109.5 \\
\hline $\mathrm{H}(26 \mathrm{~A})-\mathrm{C}(26)-\mathrm{H}(26 \mathrm{C})$ & 109.5 \\
\hline H(26B)-C(26)-H(26C) & 109.5 \\
\hline C(16)-O(27)-C(28) & $115.52(19)$ \\
\hline $\mathrm{O}(27)-\mathrm{C}(28)-\mathrm{C}(29)$ & 107.9(2) \\
\hline $\mathrm{O}(27)-\mathrm{C}(28)-\mathrm{H}(28 \mathrm{~A})$ & 110.1 \\
\hline C(29)-C(28)-H(28A) & 110.1 \\
\hline $\mathrm{O}(27)-\mathrm{C}(28)-\mathrm{H}(28 \mathrm{~B})$ & 110.1 \\
\hline C(29)-C(28)-H(28B) & 110.1 \\
\hline H(28A)-C(28)-H(28B) & 108.4 \\
\hline $\mathrm{C}(28)-\mathrm{C}(29)-\mathrm{H}(29 \mathrm{~A})$ & 109.5 \\
\hline C(28)-C(29)-H(29B) & 109.5 \\
\hline
\end{tabular}




$\begin{array}{ll}\mathrm{H}(29 \mathrm{~A})-\mathrm{C}(29)-\mathrm{H}(29 B) & 109.5 \\ \mathrm{C}(28)-\mathrm{C}(29)-\mathrm{H}(29 \mathrm{C}) & 109.5 \\ \mathrm{H}(29 \mathrm{~A})-\mathrm{C}(29)-\mathrm{H}(29 \mathrm{C}) & 109.5 \\ \mathrm{H}(29 \mathrm{~B})-\mathrm{C}(29)-\mathrm{H}(29 \mathrm{C}) & 109.5 \\ \mathrm{C}(31)-\mathrm{O}(30)-\mathrm{C}(1) & 116.80(17) \\ \mathrm{O}(32)-\mathrm{C}(31)-\mathrm{O}(30) & 119.4(2) \\ \mathrm{O}(32)-\mathrm{C}(31)-\mathrm{C}(11) & 125.4(2) \\ \text { O(30)-C(31)-C(11) } & 115.2(2)\end{array}$

Symmetry transformations used to generate equivalent atoms:

Table 4. Anisotropic displacement parameters $\left(\AA^{2} \times 10^{3}\right)$ for SP2-122. The anisotropic displacement factor exponent takes the form: $-2 \pi^{2}\left[h^{2} a^{* 2} U^{11}+\ldots+2 h k a^{*} b^{*} U^{12}\right]$

\begin{tabular}{|c|c|c|c|c|c|c|}
\hline & $\mathrm{U}^{11}$ & $\mathrm{U}^{22}$ & $\mathrm{U}^{33}$ & $\mathrm{U}^{23}$ & $\mathrm{U}^{13}$ & $\mathrm{U}^{12}$ \\
\hline $\mathrm{C}(1)$ & $27(1)$ & $36(2)$ & $37(2)$ & $-8(1)$ & $-5(1)$ & $-2(1)$ \\
\hline$C(7)$ & $26(1)$ & $34(1)$ & 28(1) & $-4(1)$ & $0(1)$ & $-3(1)$ \\
\hline C(8) & $25(1)$ & $24(1)$ & 23(1) & $-4(1)$ & $-1(1)$ & $-2(1)$ \\
\hline C(9) & $26(1)$ & 23(1) & 22(1) & $-3(1)$ & $-2(1)$ & $4(1)$ \\
\hline$C(10)$ & $26(1)$ & $25(1)$ & $28(1)$ & $-1(1)$ & $-4(1)$ & $3(1)$ \\
\hline $\mathrm{C}(11)$ & $26(1)$ & $26(1)$ & 22(1) & $0(1)$ & $-1(1)$ & $-2(1)$ \\
\hline $\mathrm{C}(12)$ & $27(1)$ & $25(1)$ & 20(1) & $3(1)$ & $-1(1)$ & $-1(1)$ \\
\hline C(13) & $24(1)$ & $28(1)$ & $22(1)$ & $-3(1)$ & $-3(1)$ & $-1(1)$ \\
\hline$C(14)$ & $25(1)$ & $20(1)$ & $28(1)$ & $-2(1)$ & $-3(1)$ & $4(1)$ \\
\hline C(15) & $24(1)$ & $34(2)$ & 28(1) & $-3(1)$ & $-3(1)$ & $0(1)$ \\
\hline$C(16)$ & $26(1)$ & $36(2)$ & 29(1) & $-2(1)$ & $4(1)$ & $-4(1)$ \\
\hline $\mathrm{O}(17)$ & $35(1)$ & $48(1)$ & $22(1)$ & $2(1)$ & $-1(1)$ & $-5(1)$ \\
\hline C(19) & $35(1)$ & $41(2)$ & $36(2)$ & $-9(1)$ & $-5(1)$ & $-6(1)$ \\
\hline$C(21)$ & $41(1)$ & $38(2)$ & $37(2)$ & 11(1) & $0(1)$ & $8(1)$ \\
\hline C(22) & $27(1)$ & $26(1)$ & 28(1) & $1(1)$ & $-1(1)$ & $1(1)$ \\
\hline $\mathrm{O}(23)$ & $45(1)$ & 39(1) & $26(1)$ & 2(1) & $7(1)$ & $-9(1)$ \\
\hline $\mathrm{O}(24)$ & $37(1)$ & $37(1)$ & 23(1) & $-8(1)$ & $2(1)$ & $-10(1)$ \\
\hline C(25) & $37(1)$ & $44(2)$ & $20(1)$ & $-10(1)$ & $-1(1)$ & $-7(1)$ \\
\hline C(26) & $47(1)$ & $41(2)$ & $31(2)$ & $-9(1)$ & $6(1)$ & $0(1)$ \\
\hline $\mathrm{O}(27)$ & $47(1)$ & $54(1)$ & $31(1)$ & $-7(1)$ & $11(1)$ & $-23(1)$ \\
\hline C(28) & $48(2)$ & 69(2) & $30(2)$ & $-11(1)$ & 15(1) & $-20(2)$ \\
\hline C(29) & $167(4)$ & $186(5)$ & 63(3) & $-46(3)$ & 68(3) & $-134(4)$ \\
\hline $\mathrm{O}(30)$ & 24(1) & 49(1) & $38(1)$ & $-11(1)$ & $-2(1)$ & $5(1)$ \\
\hline C(31) & $30(1)$ & $40(2)$ & 21(1) & $-4(1)$ & $0(1)$ & $3(1)$ \\
\hline
\end{tabular}


Table 5. Hydrogen coordinates ( $\times 10^{4}$ ) and isotropic displacement parameters $\left(\AA^{2} \times 10^{3}\right)$ for SP2-122.

\begin{tabular}{|c|c|c|c|c|}
\hline & $\mathrm{x}$ & $\mathrm{y}$ & $\mathrm{z}$ & $\mathrm{U}(\mathrm{eq})$ \\
\hline $\mathrm{H}(1 \mathrm{~A})$ & 9213 & 6241 & 2071 & 40 \\
\hline $\mathrm{H}(1 \mathrm{~B})$ & 10124 & 5701 & 2701 & 40 \\
\hline $\mathrm{H}(7)$ & 6150 & 4215 & 3743 & 35 \\
\hline $\mathrm{H}(11)$ & 7119 & 5298 & 1532 & 29 \\
\hline $\mathrm{H}(12)$ & 5598 & 2875 & 1813 & 29 \\
\hline $\mathrm{H}(13)$ & 3470 & 3989 & 1500 & 30 \\
\hline $\mathrm{H}(14)$ & 3618 & 5719 & 2481 & 29 \\
\hline $\mathrm{H}(15 \mathrm{~A})$ & 2017 & 3917 & 2486 & 34 \\
\hline $\mathrm{H}(15 \mathrm{~B})$ & 3291 & 2897 & 2657 & 34 \\
\hline $\mathrm{H}(16)$ & 2284 & 4966 & 3541 & 36 \\
\hline $\mathrm{H}(19 \mathrm{~A})$ & 7112 & 6370 & 3696 & 55 \\
\hline $\mathrm{H}(19 \mathrm{~B})$ & 8645 & 6969 & 3492 & 55 \\
\hline $\mathrm{H}(19 \mathrm{C})$ & 8540 & 5527 & 3839 & 55 \\
\hline $\mathrm{H}(21 \mathrm{~A})$ & 3331 & 6287 & 1321 & 58 \\
\hline $\mathrm{H}(21 \mathrm{~B})$ & 4428 & 5655 & 786 & 58 \\
\hline $\mathrm{H}(21 \mathrm{C})$ & 5025 & 6443 & 1432 & 58 \\
\hline $\mathrm{H}(25 \mathrm{~A})$ & 4993 & 2790 & -347 & 41 \\
\hline $\mathrm{H}(25 \mathrm{~B})$ & 4141 & 1450 & -159 & 41 \\
\hline $\mathrm{H}(26 \mathrm{~A})$ & 7171 & 1709 & -211 & 60 \\
\hline $\mathrm{H}(26 \mathrm{~B})$ & 6209 & 855 & -724 & 60 \\
\hline $\mathrm{H}(26 \mathrm{C})$ & 6375 & 385 & 46 & 60 \\
\hline $\mathrm{H}(28 \mathrm{~A})$ & 816 & 4197 & 4415 & 59 \\
\hline $\mathrm{H}(28 \mathrm{~B})$ & 2070 & 3272 & 4718 & 59 \\
\hline $\mathrm{H}(29 \mathrm{~A})$ & -630 & 2346 & 4239 & 208 \\
\hline $\mathrm{H}(29 \mathrm{~B})$ & -104 & 2312 & 5010 & 208 \\
\hline $\mathrm{H}(29 \mathrm{C})$ & 640 & 1362 & 4460 & 208 \\
\hline
\end{tabular}




\section{Lactone 6b (CCDC 600923)}<smiles>[CH]C1C2=C(C)COC(=O)[C@H](C(C)=O)C2=COC[C@@H]1OCC</smiles>

Experimental : The crystals were grown by vapor diffusion of hexanes on a dichloromethane saturated solution at room temperature. One single crystal of 0.5 X 0.3 X $0.3 \mathrm{~mm}$ was mounted using a glass fiber on the goniometer at 293(2) K. Data were collected on an Enraf-Nonius CAD-4 automatic diffractometer at the Université de Sherbrooke using $\omega / 2$ theta scans. The DIFRAC ${ }^{(1)}$ program was used for centering, indexing, and data collection. Two standard reflections were measured every 100 reflections, an intensity decay of $23.6 \%$ was observed during data collection. The data were corrected for absorption by empirical methods based on psi scans and reduced with the NRCVAX ${ }^{(2)}$ programs. They were solved using SHELXS-97 $7^{(3)}$ and refined by full-matrix least squares on $\mathrm{F}^{2}$ with SHELXL-97 ${ }^{(4)}$. The non-hydrogen atoms were refined anisotropically. The hydrogen atoms were placed at idealized calculated geometric position and refined isotropically using a riding model. The worse reflection profiles were omitted in SHELXL-97 to improve the residual and goodness of fit.

(1) H.D. Flack, E. Blanc and D. Schwarzenbach (1992), J. Appl. Cryst., 25, 455-459.

(2) E.J. Gabe, Y. Le Page, J.-P. Charland, F.L. Lee, and P.S. White, (1989) J. Appl. Cryst., 22, 384-387.

(3) G. M. Sheldrick, SHELXS-97, G.M. Sheldrick, University of Göttingen, Germany, 1997, Release 97-2.

(4) G. M. Sheldrick, SHELXL-97, G.M. Sheldrick, University of Göttingen, Germany, 1997, Release 97-2. 


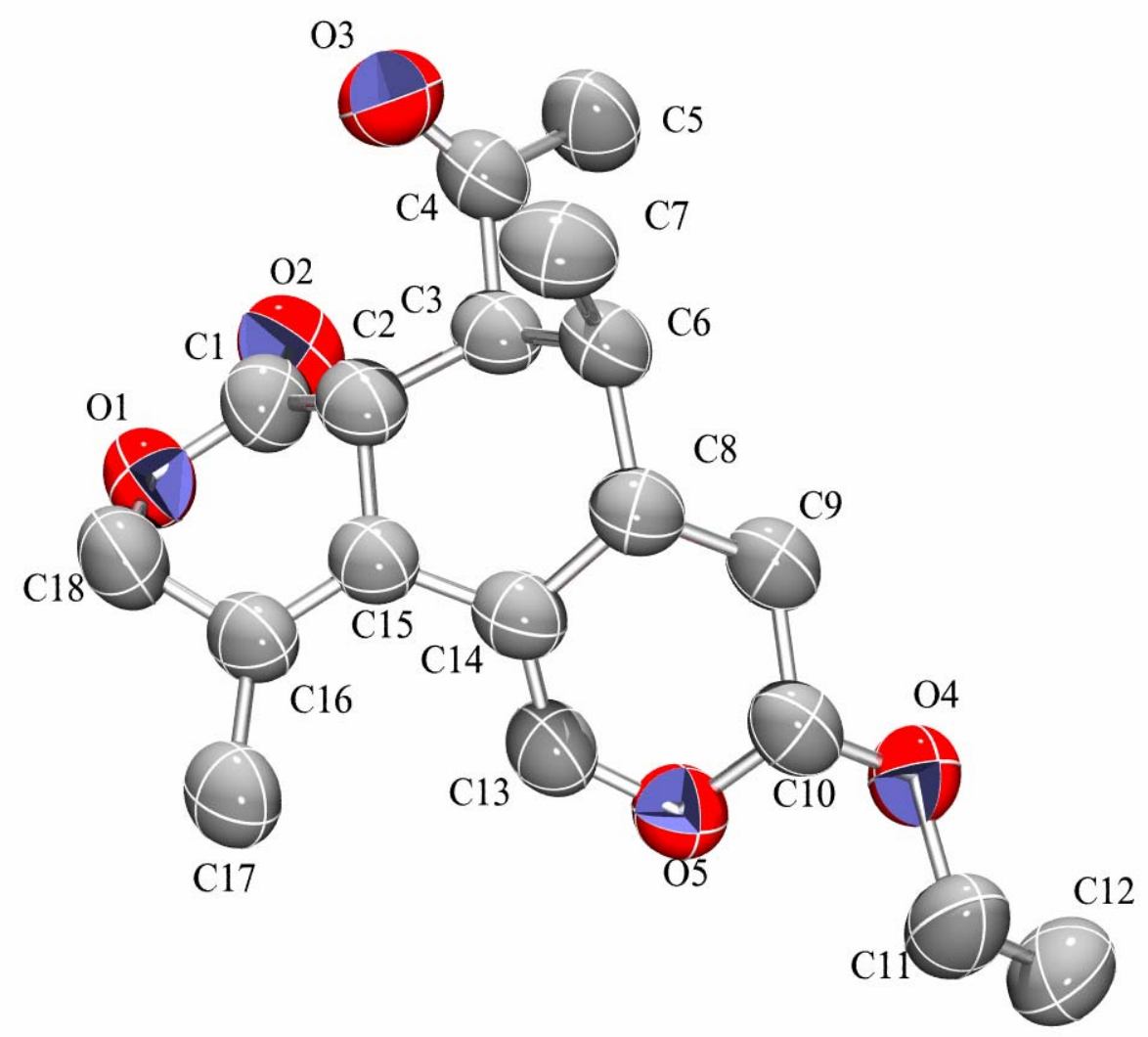

Ellipsoid probability set at 50\%.

Table 1. Crystal data and structure refinement for sp5029.

Identification code

sp5029

Empirical formula

C18 H24 O5

Formula weight

320.37

Temperature

293(2) K

Wavelength

$1.54176 \AA$

Crystal system

Monoclinic

Space group

Unit cell dimensions

p121/n1

$\mathrm{a}=11.660(13) \AA$

$\alpha=90^{\circ}$.

$\mathrm{b}=12.284(11) \AA$

$\beta=115.01(9)^{\circ}$.

c $=12.953(17) \AA$

$\gamma=90^{\circ}$.

Volume

1681(3) $\AA^{3}$

Z

4

Density (calculated)

$1.266 \mathrm{Mg} / \mathrm{m}^{3}$

Absorption coefficient

$0.750 \mathrm{~mm}^{-1}$

$\mathrm{F}(000)$

688 
Crystal size

Theta range for data collection

Index ranges

Reflections collected

Independent reflections

Completeness to theta $=69.91^{\circ}$

Absorption correction

Max. and min. transmission

Refinement method

Data / restraints / parameters

Goodness-of-fit on $\mathrm{F}^{2}$

Final R indices [I $>2 \operatorname{sigma(I)]~}$

$\mathrm{R}$ indices (all data)

Extinction coefficient

Largest diff. peak and hole
$0.50 \times 0.30 \times 0.30 \mathrm{~mm}^{3}$

4.29 to $69.91^{\circ}$.

$-14<=\mathrm{h}<=12,0<=\mathrm{k}<=14,0<=\mathrm{l}<=15$

2822

$2822[\mathrm{R}(\mathrm{int})=0.0000]$

$88.7 \%$

Empirical

0.8062 and 0.7054

Full-matrix least-squares on $\mathrm{F}^{2}$

2822 / 0 / 237

0.973

$\mathrm{R} 1=0.0871, \mathrm{wR} 2=0.2414$

$\mathrm{R} 1=0.1364, \mathrm{wR} 2=0.2845$

$0.009(2)$

0.213 and -0.215 e. $\AA^{-3}$

Table 2. Atomic coordinates ( $\left.\mathrm{x} 10^{4}\right)$ and equivalent isotropic displacement parameters $\left(\AA^{2} \mathrm{x} 10^{3}\right)$ for sp5029. $U(e q)$ is defined as one third of the trace of the orthogonalized $U^{i j}$ tensor.

\begin{tabular}{|c|c|c|c|c|}
\hline & $\mathrm{x}$ & $\mathrm{y}$ & $\mathrm{z}$ & $\mathrm{U}(\mathrm{eq})$ \\
\hline$C(6)$ & $3344(4)$ & $6390(3)$ & 989(3) & $76(1)$ \\
\hline $\mathrm{C}(8)$ & $4214(4)$ & 6308(3) & $364(3)$ & $76(1)$ \\
\hline$C(1)$ & $146(4)$ & $6346(4)$ & $-1664(3)$ & $89(1)$ \\
\hline $\mathrm{C}(2)$ & $1404(4)$ & 5964(3) & $-793(3)$ & $78(1)$ \\
\hline C(3) & 1990(3) & $6770(3)$ & $160(3)$ & $74(1)$ \\
\hline$C(4)$ & $1205(4)$ & 6988(3) & $817(3)$ & $84(1)$ \\
\hline$C(5)$ & 1411(5) & $8055(4)$ & $1440(4)$ & $102(1)$ \\
\hline$C(7)$ & $3303(5)$ & $5313(4)$ & 1592(4) & $99(1)$ \\
\hline C(9) & 5089(4) & 7288(3) & $592(3)$ & $80(1)$ \\
\hline$C(10)$ & $5858(4)$ & 7201(3) & $-77(3)$ & $82(1)$ \\
\hline $\mathrm{C}(11)$ & $7571(5)$ & 7963(5) & $-345(6)$ & $117(2)$ \\
\hline $\mathrm{C}(12)$ & 8181(6) & $9024(5)$ & $-317(5)$ & $117(2)$ \\
\hline$C(13)$ & 3936(4) & 6633(3) & $-1598(3)$ & 83(1) \\
\hline$C(14)$ & $3492(4)$ & $6171(3)$ & $-908(3)$ & $78(1)$ \\
\hline$C(15)$ & 2226(4) & 5673(3) & $-1392(3)$ & $74(1)$ \\
\hline$C(16)$ & $1702(4)$ & $5050(3)$ & $-2332(3)$ & $80(1)$ \\
\hline $\mathrm{C}(17)$ & $2327(5)$ & $4570(3)$ & $-3022(4)$ & 93(1) \\
\hline $\mathrm{C}(18)$ & $326(4)$ & $4804(4)$ & $-2718(4)$ & $98(1)$ \\
\hline
\end{tabular}




\begin{tabular}{lrrrr}
$\mathrm{O}(1)$ & $-374(3)$ & $5733(2)$ & $-2609(2)$ & $96(1)$ \\
$\mathrm{O}(2)$ & $-371(3)$ & $7177(3)$ & $-1595(2)$ & $111(1)$ \\
$\mathrm{O}(3)$ & $484(3)$ & $6322(3)$ & $865(3)$ & $107(1)$ \\
$\mathrm{O}(4)$ & $6644(3)$ & $8098(2)$ & $102(2)$ & $94(1)$ \\
$\mathrm{O}(5)$ & $5049(3)$ & $7175(2)$ & $-1292(2)$ & $92(1)$ \\
\hline
\end{tabular}

Table 3. Bond lengths $[\AA]$ and angles $\left[{ }^{\circ}\right]$ for sp5029.

\begin{tabular}{lr}
\hline $\mathrm{C}(6)-\mathrm{C}(8)$ & $1.545(5)$ \\
$\mathrm{C}(6)-\mathrm{C}(7)$ & $1.547(6)$ \\
$\mathrm{C}(6)-\mathrm{C}(3)$ & $1.558(5)$ \\
$\mathrm{C}(8)-\mathrm{C}(14)$ & $1.510(5)$ \\
$\mathrm{C}(8)-\mathrm{C}(9)$ & $1.524(5)$ \\
$\mathrm{C}(1)-\mathrm{O}(2)$ & $1.208(5)$ \\
$\mathrm{C}(1)-\mathrm{O}(1)$ & $1.344(5)$ \\
$\mathrm{C}(1)-\mathrm{C}(2)$ & $1.498(6)$ \\
$\mathrm{C}(2)-\mathrm{C}(3)$ & $1.503(5)$ \\
$\mathrm{C}(2)-\mathrm{C}(15)$ & $1.510(5)$ \\
$\mathrm{C}(3)-\mathrm{C}(4)$ & $1.514(5)$ \\
$\mathrm{C}(4)-\mathrm{O}(3)$ & $1.194(5)$ \\
$\mathrm{C}(4)-\mathrm{C}(5)$ & $1.504(6)$ \\
$\mathrm{C}(9)-\mathrm{C}(10)$ & $1.491(5)$ \\
$\mathrm{C}(10)-\mathrm{O}(4)$ & $1.389(4)$ \\
$\mathrm{C}(10)-\mathrm{O}(5)$ & $1.453(5)$ \\
$\mathrm{C}(11)-\mathrm{O}(4)$ & $1.435(5)$ \\
$\mathrm{C}(11)-\mathrm{C}(12)$ & $1.478(6)$ \\
$\mathrm{C}(13)-\mathrm{C}(14)$ & $1.334(5)$ \\
$\mathrm{C}(13)-\mathrm{O}(5)$ & $1.361(5)$ \\
$\mathrm{C}(14)-\mathrm{C}(15)$ & $1.470(6)$ \\
$\mathrm{C}(15)-\mathrm{C}(16)$ & $1.346(5)$ \\
$\mathrm{C}(16)-\mathrm{C}(17)$ & $1.493(6)$ \\
$\mathrm{C}(16)-\mathrm{C}(18)$ & $1.496(6)$ \\
$\mathrm{C}(18)-\mathrm{O}(1)$ & $1.444(5)$ \\
$\mathrm{C}(8)-\mathrm{C}(6)-\mathrm{C}(7)$ & \\
$\mathrm{C}(8)-\mathrm{C}(6)-\mathrm{C}(3)$ & $112.1(3)$ \\
$\mathrm{C}(7)-\mathrm{C}(6)-\mathrm{C}(3)$ & \\
$\mathrm{C}(14)-\mathrm{C}(8)-\mathrm{C}(9)$ & \\
$\mathrm{C}(14)-\mathrm{C}(8)-\mathrm{C}(6)$ & \\
& \\
& \\
&
\end{tabular}




$\begin{array}{ll}\mathrm{C}(9)-\mathrm{C}(8)-\mathrm{C}(6) & 112.4(3) \\ \mathrm{O}(2)-\mathrm{C}(1)-\mathrm{O}(1) & 119.6(4) \\ \mathrm{O}(2)-\mathrm{C}(1)-\mathrm{C}(2) & 124.7(4) \\ \mathrm{O}(1)-\mathrm{C}(1)-\mathrm{C}(2) & 115.5(4) \\ \mathrm{C}(1)-\mathrm{C}(2)-\mathrm{C}(3) & 111.9(4) \\ \mathrm{C}(1)-\mathrm{C}(2)-\mathrm{C}(15) & 108.7(3) \\ \mathrm{C}(3)-\mathrm{C}(2)-\mathrm{C}(15) & 114.3(3) \\ \mathrm{C}(2)-\mathrm{C}(3)-\mathrm{C}(4) & 113.9(3) \\ \mathrm{C}(2)-\mathrm{C}(3)-\mathrm{C}(6) & 110.2(3) \\ \mathrm{C}(4)-\mathrm{C}(3)-\mathrm{C}(6) & 110.0(3) \\ \mathrm{O}(3)-\mathrm{C}(4)-\mathrm{C}(5) & 121.7(4) \\ \mathrm{O}(3)-\mathrm{C}(4)-\mathrm{C}(3) & 121.0(3) \\ \mathrm{C}(5)-\mathrm{C}(4)-\mathrm{C}(3) & 117.2(4) \\ \mathrm{C}(10)-\mathrm{C}(9)-\mathrm{C}(8) & 110.4(3) \\ \mathrm{O}(4)-\mathrm{C}(10)-\mathrm{O}(5) & 106.2(3) \\ \mathrm{O}(4)-\mathrm{C}(10)-\mathrm{C}(9) & 111.3(3) \\ \mathrm{O}(5)-\mathrm{C}(10)-\mathrm{C}(9) & 110.9(3) \\ \mathrm{O}(4)-\mathrm{C}(11)-\mathrm{C}(12) & 108.9(4) \\ \mathrm{C}(14)-\mathrm{C}(13)-\mathrm{O}(5) & 127.3(4) \\ \mathrm{C}(13)-\mathrm{C}(14)-\mathrm{C}(15) & 119.6(3) \\ \mathrm{C}(13)-\mathrm{C}(14)-\mathrm{C}(8) & 119.2(4) \\ \mathrm{C}(15)-\mathrm{C}(14)-\mathrm{C}(8) & 120.5(3) \\ \mathrm{C}(16)-\mathrm{C}(15)-\mathrm{C}(14) & 127.8(4) \\ \mathrm{C}(16)-\mathrm{C}(15)-\mathrm{C}(2) & 117.1(3) \\ \mathrm{C}(14)-\mathrm{C}(15)-\mathrm{C}(2) & 115.0(3) \\ \mathrm{C}(15)-\mathrm{C}(16)-\mathrm{C}(17) & 128.0(4) \\ \mathrm{C}(15)-\mathrm{C}(16)-\mathrm{C}(18) & 115.5(4) \\ \mathrm{C}(17)-\mathrm{C}(16)-\mathrm{C}(18) & 116.5(3) \\ \mathrm{O}(1)-\mathrm{C}(18)-\mathrm{C}(16) & 112.2(3) \\ \mathrm{C}(1)-\mathrm{O}(1)-\mathrm{C}(18) & 118.5(3) \\ \mathrm{C}(10)-\mathrm{O}(4)-\mathrm{C}(11) & 113.4(3) \\ \mathrm{C}(13)-\mathrm{O}(5)-\mathrm{C}(10) & \end{array}$

Symmetry transformations used to generate equivalent atoms: 
Table 4. Anisotropic displacement parameters $\left(\AA^{2} \times 10^{3}\right)$ for sp5029. The anisotropic displacement factor exponent takes the form: $-2 \pi^{2}\left[h^{2} a^{* 2} U^{11}+\ldots+2 h k a^{*} b^{*} U^{12}\right]$

\begin{tabular}{|c|c|c|c|c|c|c|}
\hline & $\mathrm{U}^{11}$ & $\mathrm{U}^{22}$ & $\mathrm{U}^{33}$ & $\mathrm{U}^{23}$ & $\mathrm{U}^{13}$ & $\mathrm{U}^{12}$ \\
\hline$C(6)$ & $90(2)$ & $80(2)$ & $59(2)$ & $0(2)$ & $33(2)$ & $1(2)$ \\
\hline C(8) & $86(2)$ & $81(2)$ & $65(2)$ & $7(2)$ & $36(2)$ & $2(2)$ \\
\hline $\mathrm{C}(1)$ & 89(3) & $95(3)$ & $76(2)$ & $5(2)$ & $29(2)$ & $-3(2)$ \\
\hline $\mathrm{C}(2)$ & 103(3) & $72(2)$ & $64(2)$ & $8(2)$ & $40(2)$ & $-10(2)$ \\
\hline C(3) & $99(2)$ & $66(2)$ & 64(2) & $7(2)$ & $40(2)$ & $-5(2)$ \\
\hline C(4) & 99(3) & $86(2)$ & $67(2)$ & $-1(2)$ & $36(2)$ & $2(2)$ \\
\hline$C(5)$ & $113(4)$ & $97(3)$ & $100(3)$ & $-23(3)$ & 51(3) & $3(2)$ \\
\hline$C(7)$ & $127(4)$ & $98(3)$ & $83(2)$ & $21(2)$ & $55(3)$ & $8(3)$ \\
\hline C(9) & $92(2)$ & $76(2)$ & $72(2)$ & $-5(2)$ & $36(2)$ & $6(2)$ \\
\hline$C(10)$ & $92(2)$ & $80(2)$ & $76(2)$ & $-8(2)$ & $37(2)$ & $5(2)$ \\
\hline$C(11)$ & 102(3) & $124(4)$ & $145(5)$ & $-36(4)$ & 72(3) & $-15(3)$ \\
\hline $\mathrm{C}(12)$ & $113(4)$ & $126(4)$ & $127(4)$ & $8(3)$ & 64(3) & $-20(3)$ \\
\hline C(13) & 99(3) & $77(2)$ & $74(2)$ & $-6(2)$ & $37(2)$ & $-5(2)$ \\
\hline C(14) & $98(3)$ & $73(2)$ & $69(2)$ & $1(2)$ & $42(2)$ & $-2(2)$ \\
\hline$C(15)$ & $91(2)$ & $66(2)$ & $68(2)$ & $3(2)$ & $39(2)$ & $-4(2)$ \\
\hline$C(16)$ & 105(3) & $69(2)$ & $76(2)$ & $-7(2)$ & $47(2)$ & $-11(2)$ \\
\hline $\mathrm{C}(17)$ & $115(3)$ & $76(2)$ & 89(3) & $-7(2)$ & $45(3)$ & $-12(2)$ \\
\hline $\mathrm{C}(18)$ & $114(3)$ & $100(3)$ & $78(2)$ & $-17(2)$ & $37(2)$ & $-17(3)$ \\
\hline $\mathrm{O}(1)$ & $95(2)$ & $109(2)$ & $80(2)$ & $-9(1)$ & $32(2)$ & $-4(2)$ \\
\hline $\mathrm{O}(2)$ & $108(2)$ & $118(2)$ & 93(2) & $7(2)$ & $29(2)$ & $29(2)$ \\
\hline O(3) & $123(2)$ & $103(2)$ & $119(2)$ & $-10(2)$ & $75(2)$ & $-17(2)$ \\
\hline $\mathrm{O}(4)$ & $95(2)$ & $91(2)$ & $102(2)$ & $-19(2)$ & $49(2)$ & $-16(1)$ \\
\hline $\mathrm{O}(5)$ & $105(2)$ & $98(2)$ & $80(2)$ & $-4(1)$ & $46(1)$ & $-15(2)$ \\
\hline
\end{tabular}

Table 5. Hydrogen coordinates ( $\left.\mathrm{x} 10^{4}\right)$ and isotropic displacement parameters $\left(\AA^{2} \times 10^{3}\right)$ for sp5029.

\begin{tabular}{lrrrr}
\hline & $x$ & $y$ & $z$ & $U(e q)$ \\
\hline$H(6)$ & 3699 & 6949 & 1579 & $59(8)$ \\
$H(8)$ & 4748 & 5662 & 656 & $72(9)$ \\
$H(2)$ & 1256 & 5293 & -459 & $46(6)$ \\
$H(3)$ & 2078 & 7461 & -178 & $59(8)$
\end{tabular}




\begin{tabular}{lrrrr}
$\mathrm{H}(5 \mathrm{~A})$ & 812 & 8126 & 1765 & $190(30)$ \\
$\mathrm{H}(5 \mathrm{~B})$ & 1298 & 8642 & 917 & $150(20)$ \\
$\mathrm{H}(5 \mathrm{C})$ & 2255 & 8077 & 2035 & $170(30)$ \\
$\mathrm{H}(7 \mathrm{~A})$ & 2950 & 4748 & 1035 & $77(10)$ \\
$\mathrm{H}(7 \mathrm{~B})$ & 2788 & 5409 & 2000 & $230(40)$ \\
$\mathrm{H}(7 \mathrm{C})$ & 4146 & 5115 & 2116 & $200(30)$ \\
$\mathrm{H}(9 \mathrm{~A})$ & 4589 & 7950 & 383 & $82(11)$ \\
$\mathrm{H}(9 \mathrm{~B})$ & 5646 & 7326 & 1398 & $131(16)$ \\
$\mathrm{H}(10)$ & 6370 & 6537 & 143 & $69(9)$ \\
$\mathrm{H}(11 \mathrm{~A})$ & 7168 & 7696 & -1122 & $134(18)$ \\
$\mathrm{H}(11 \mathrm{~B})$ & 8202 & 7436 & 109 & $160(20)$ \\
$\mathrm{H}(12 \mathrm{~A})$ & 7551 & 9542 & -766 & $200(30)$ \\
$\mathrm{H}(12 \mathrm{~B})$ & 8797 & 8942 & -621 & $140(20)$ \\
$\mathrm{H}(12 \mathrm{C})$ & 8590 & 9277 & 456 & $200(30)$ \\
$\mathrm{H}(13)$ & 3427 & 6578 & -2375 & $101(13)$ \\
$\mathrm{H}(17 \mathrm{~A})$ & 2044 & 4944 & -3738 & $69(9)$ \\
$\mathrm{H}(17 \mathrm{~B})$ & 2112 & 3813 & -3155 & $88(11)$ \\
$\mathrm{H}(17 \mathrm{C})$ & 3229 & 4644 & -2616 & $113(15)$ \\
$\mathrm{H}(18 \mathrm{~A})$ & -27 & 4575 & -3509 & $114(14)$ \\
$\mathrm{H}(18 \mathrm{~B})$ & 232 & 4207 & -2270 & $75(10)$ \\
\hline
\end{tabular}

Table 6. Torsion angles $\left[^{\circ}\right]$ for sp5029.

\begin{tabular}{lc}
\hline$C(7)-C(6)-C(8)-C(14)$ & $102.6(4)$ \\
$C(3)-C(6)-C(8)-C(14)$ & $-22.7(4)$ \\
$C(7)-C(6)-C(8)-C(9)$ & $-134.0(4)$ \\
$C(3)-C(6)-C(8)-C(9)$ & $100.7(3)$ \\
$O(2)-C(1)-C(2)-C(3)$ & $-2.9(6)$ \\
$O(1)-C(1)-C(2)-C(3)$ & $172.6(3)$ \\
$O(2)-C(1)-C(2)-C(15)$ & $-130.0(4)$ \\
$O(1)-C(1)-C(2)-C(15)$ & $45.4(4)$ \\
$C(1)-C(2)-C(3)-C(4)$ & $60.7(4)$ \\
$C(15)-C(2)-C(3)-C(4)$ & $-175.3(3)$ \\
$C(1)-C(2)-C(3)-C(6)$ & $-175.1(3)$ \\
$C(15)-C(2)-C(3)-C(6)$ & $-51.1(4)$ \\
$C(8)-C(6)-C(3)-C(2)$ & $61.1(4)$ \\
$C(7)-C(6)-C(3)-C(2)$ & $-64.6(4)$ \\
$C(8)-C(6)-C(3)-C(4)$ & $-172.6(3)$ \\
$C(7)-C(6)-C(3)-C(4)$ & $61.8(4)$
\end{tabular}




\begin{tabular}{|c|c|}
\hline $\mathrm{C}(2)-\mathrm{C}(3)-\mathrm{C}(4)-\mathrm{O}(3)$ & $26.2(5)$ \\
\hline $\mathrm{C}(6)-\mathrm{C}(3)-\mathrm{C}(4)-\mathrm{O}(3)$ & $-98.1(4)$ \\
\hline C(2)-C(3)-C(4)-C(5) & $-156.1(4)$ \\
\hline $\mathrm{C}(6)-\mathrm{C}(3)-\mathrm{C}(4)-\mathrm{C}(5)$ & $79.6(4)$ \\
\hline C(14)-C(8)-C(9)-C(10) & $-51.1(4)$ \\
\hline C(6)-C(8)-C(9)-C(10) & $-176.9(3)$ \\
\hline C(8)-C(9)-C(10)-O(4) & $178.8(3)$ \\
\hline C(8)-C(9)-C(10)-O(5) & $60.9(4)$ \\
\hline $\mathrm{O}(5)-\mathrm{C}(13)-\mathrm{C}(14)-\mathrm{C}(15)$ & $176.4(3)$ \\
\hline $\mathrm{O}(5)-\mathrm{C}(13)-\mathrm{C}(14)-\mathrm{C}(8)$ & $5.1(6)$ \\
\hline C(9)-C(8)-C(14)-C(13) & $19.6(5)$ \\
\hline C(6)-C(8)-C(14)-C(13) & $145.1(4)$ \\
\hline$C(9)-C(8)-C(14)-C(15)$ & $-151.6(3)$ \\
\hline C(6)-C(8)-C(14)-C(15) & $-26.2(5)$ \\
\hline $\mathrm{C}(13)-\mathrm{C}(14)-\mathrm{C}(15)-\mathrm{C}(16)$ & $42.0(6)$ \\
\hline C(8)-C(14)-C(15)-C(16) & $-146.8(4)$ \\
\hline C(13)-C(14)-C(15)-C(2) & $-133.8(4)$ \\
\hline $\mathrm{C}(8)-\mathrm{C}(14)-\mathrm{C}(15)-\mathrm{C}(2)$ & $37.4(5)$ \\
\hline $\mathrm{C}(1)-\mathrm{C}(2)-\mathrm{C}(15)-\mathrm{C}(16)$ & $-47.1(4)$ \\
\hline C(3)-C(2)-C(15)-C(16) & $-172.8(3)$ \\
\hline C(1)-C(2)-C(15)-C(14) & $129.2(4)$ \\
\hline C(3)-C(2)-C(15)-C(14) & $3.4(4)$ \\
\hline $\mathrm{C}(14)-\mathrm{C}(15)-\mathrm{C}(16)-\mathrm{C}(17)$ & $10.0(6)$ \\
\hline C(2)-C(15)-C(16)-C(17) & $-174.3(3)$ \\
\hline $\mathrm{C}(14)-\mathrm{C}(15)-\mathrm{C}(16)-\mathrm{C}(18)$ & $-170.7(4)$ \\
\hline C(2)-C(15)-C(16)-C(18) & $5.0(5)$ \\
\hline C(15)-C(16)-C(18)-O(1) & $39.9(5)$ \\
\hline $\mathrm{C}(17)-\mathrm{C}(16)-\mathrm{C}(18)-\mathrm{O}(1)$ & $-140.7(4)$ \\
\hline $\mathrm{O}(2)-\mathrm{C}(1)-\mathrm{O}(1)-\mathrm{C}(18)$ & $173.8(4)$ \\
\hline $\mathrm{C}(2)-\mathrm{C}(1)-\mathrm{O}(1)-\mathrm{C}(18)$ & $-1.9(5)$ \\
\hline $\mathrm{C}(16)-\mathrm{C}(18)-\mathrm{O}(1)-\mathrm{C}(1)$ & $-42.0(5)$ \\
\hline $\mathrm{O}(5)-\mathrm{C}(10)-\mathrm{O}(4)-\mathrm{C}(11)$ & $-70.4(4)$ \\
\hline $\mathrm{C}(9)-\mathrm{C}(10)-\mathrm{O}(4)-\mathrm{C}(11)$ & $168.8(4)$ \\
\hline $\mathrm{C}(12)-\mathrm{C}(11)-\mathrm{O}(4)-\mathrm{C}(10)$ & $168.9(4)$ \\
\hline $\mathrm{C}(14)-\mathrm{C}(13)-\mathrm{O}(5)-\mathrm{C}(10)$ & $3.1(6)$ \\
\hline $\mathrm{O}(4)-\mathrm{C}(10)-\mathrm{O}(5)-\mathrm{C}(13)$ & $-157.0(3)$ \\
\hline $\mathrm{C}(9)-\mathrm{C}(10)-\mathrm{O}(5)-\mathrm{C}(13)$ & $-36.0(4)$ \\
\hline
\end{tabular}

Symmetry transformations used to generate equivalent atoms: 


\section{Triester 19a (CCDC 600918)}<smiles>CCOC(=O)[C@@H]1C(C)C2SC(C)(C(=O)OCC)CC(C)=C2C2=CO[C@H](OCC)C[C@H]21</smiles>

Experimental : The crystal was grown by cooling of a saturated solution using ethanol at room temperature. One single crystal was mounted using a glass fiber on the goniometer. Data were collected on an Enraf-Nonius CAD-4 automatic diffractometer at the Université de Sherbrooke using $\omega$ scans at 293(2) K. The DIFRAC ${ }^{(1)}$ program was used for centering, indexing, and data collection. Two standard reflections were measured every 100 reflections, 6.6\% intensity decay was observed during data collection. The data were reduced with the $\operatorname{NRCVAX}^{(2)}$ programs. They were solved using SHELXS-97 $7^{(3)}$ and refined by full-matrix least squares on $\mathrm{F}^{2}$ with SHELXL-97 $7^{(4)}$. The non-hydrogen atoms were refined anisotropically. The hydrogen atoms were placed at idealized calculated geometric position and refined isotropically using a riding model. Oxygen $\mathrm{O}_{24}$ was disordered on two sites $\mathrm{A}$ and $\mathrm{B}$. They were refined with an occupation sum of 1.00 . The final occupation found was $24.9 \%$ for $\mathrm{O}_{24} \mathrm{~A}$ and $75.1 \%$ for $\mathrm{O}_{24} \mathrm{~B}$. Only $\mathrm{O}_{24} \mathrm{~B}$ is shown for clarity. The $\mathrm{C}_{29}$ carbon was also disordered, the same procedure was applied and the final refined occupation found was $45.8 \%$ for $\mathrm{C}_{29} \mathrm{~A}$ and $54.2 \%$ for $\mathrm{C}_{29} \mathrm{~B}$. Only $\mathrm{C}_{29} \mathrm{~B}$ is shown for clarity. These four disordered atoms were refined using thermal (SIMU) and rigid bond (DELU) restraints.

(1) H.D. Flack, E. Blanc and D. Schwarzenbach (1992), J. Appl. Cryst., 25, 455-459.

(2) E.J. Gabe, Y. Le Page, J.-P. Charland, F.L. Lee, and P.S. White, (1989) J. Appl. Cryst., 22, 384-387.

(3) G. M. Sheldrick, SHELXS-97, G.M. Sheldrick, University of Göttingen, Germany, 1997, Release 97-2.

(4) G. M. Sheldrick, SHELXL-97, G.M. Sheldrick, University of Göttingen, Germany, 1997, Release 97-2. 


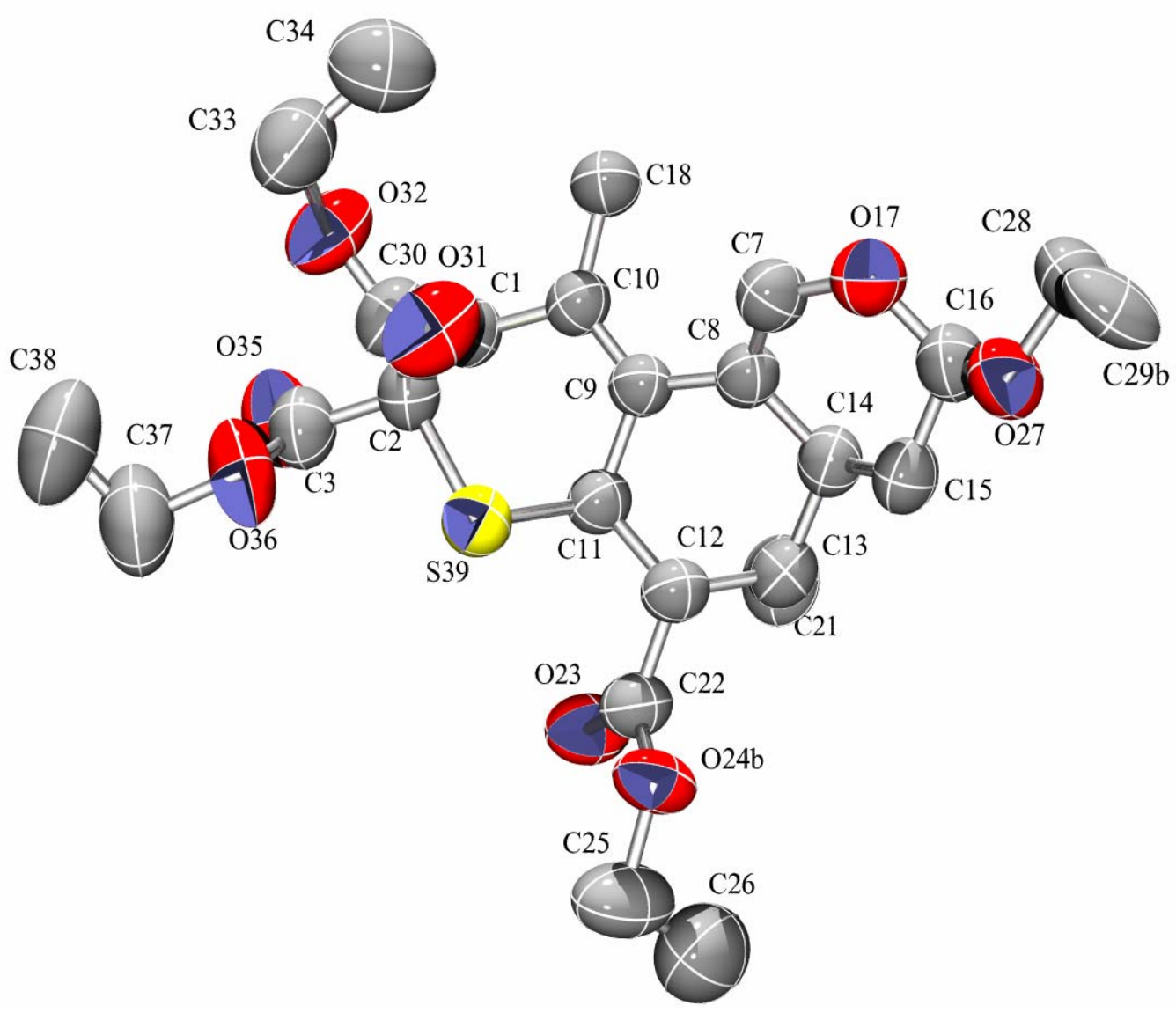

Table 1. Crystal data and structure refinement for sp054.

Identification code

Empirical formula

Formula weight

Temperature

Wavelength

Crystal system

Space group

Unit cell dimensions

Volume

Z

Density (calculated)

Absorption coefficient

$\mathrm{F}(000)$ sp054

C25 H36 O8 S

496.60

293(2) K

$1.54056 \AA$

$\mathrm{P} 2 / \mathrm{m}$

$\mathrm{P} 21 / \mathrm{n}$

$\mathrm{a}=12.3087(17) \AA$

$\alpha=90^{\circ}$.

$\mathrm{b}=9.961(2) \AA$

$\beta=90.346(10)^{\circ}$.

$\mathrm{c}=21.212(3) \AA$

$\gamma=90^{\circ}$.

2600.8(7) $\AA^{3}$

4

$1.268 \mathrm{Mg} / \mathrm{m}^{3}$

$1.488 \mathrm{~mm}^{-1}$

1064 
Crystal size

Theta range for data collection

Index ranges

Reflections collected

Independent reflections

Completeness to theta $=69.98^{\circ}$

Absorption correction

Refinement method

Data / restraints / parameters

Goodness-of-fit on $\mathrm{F}^{2}$

Final R indices [I>2sigma(I)]

$\mathrm{R}$ indices (all data)

Extinction coefficient

Largest diff. peak and hole
$0.5 \times 0.3 \times 0.2 \mathrm{~mm}^{3}$

4.14 to $69.98^{\circ}$.

$-15<=\mathrm{h}<=15,0<=\mathrm{k}<=12,0<=\mathrm{l}<=25$

4938

$4938[\mathrm{R}$ (int) $=0.0000]$

$99.9 \%$

None

Full-matrix least-squares on $\mathrm{F}^{2}$

4938 / 12 / 339

1.098

$\mathrm{R} 1=0.0770, \mathrm{wR} 2=0.2206$

$\mathrm{R} 1=0.1279, \mathrm{wR} 2=0.2538$

0.0060(7)

0.381 and -0.267 e. $\AA^{-3}$

Table 2. Atomic coordinates ( $\left.\mathrm{x} 10^{4}\right)$ and equivalent isotropic displacement parameters $\left(\AA^{2} \mathrm{x} 10^{3}\right)$ for sp054. $U(e q)$ is defined as one third of the trace of the orthogonalized $U^{i j}$ tensor.

\begin{tabular}{|c|c|c|c|c|}
\hline & $\mathrm{x}$ & $\mathrm{y}$ & $\mathrm{z}$ & $\mathrm{U}(\mathrm{eq})$ \\
\hline $\mathrm{C}(21)$ & $3811(4)$ & $10375(5)$ & $3453(2)$ & $110(2)$ \\
\hline $\mathrm{C}(1)$ & 4899(3) & $9561(4)$ & 1115(2) & $78(1)$ \\
\hline$C(2)$ & 5261(3) & $8064(4)$ & $1113(2)$ & $78(1)$ \\
\hline$C(3)$ & $6450(4)$ & $7952(6)$ & $934(2)$ & $96(1)$ \\
\hline $\mathrm{C}(7)$ & 1561(3) & $8709(4)$ & $1820(2)$ & $86(1)$ \\
\hline$C(8)$ & 2396(3) & $9115(4)$ & $2171(2)$ & $72(1)$ \\
\hline $\mathrm{C}(9)$ & 3488(3) & $9227(4)$ & 1899(2) & $69(1)$ \\
\hline$C(10)$ & 3743(3) & $9716(4)$ & 1329(2) & $72(1)$ \\
\hline $\mathrm{C}(11)$ & $4402(3)$ & $8686(4)$ & 2301(2) & $72(1)$ \\
\hline $\mathrm{C}(12)$ & 4011(3) & $8079(4)$ & $2920(2)$ & $73(1)$ \\
\hline $\mathrm{C}(13)$ & 3249(3) & $9062(4)$ & $3272(2)$ & $80(1)$ \\
\hline$C(14)$ & 2221(3) & $9295(4)$ & $2871(2)$ & $78(1)$ \\
\hline$C(15)$ & 1309(3) & $8365(5)$ & $3068(2)$ & $88(1)$ \\
\hline $\mathrm{C}(16)$ & $304(3)$ & $8540(5)$ & $2670(2)$ & $89(1)$ \\
\hline $\mathrm{C}(18)$ & 2983(3) & $10397(4)$ & $877(2)$ & $86(1)$ \\
\hline C(37) & 7918(5) & 6367(8) & 748(3) & $160(3)$ \\
\hline C(38) & $8003(6)$ & 6089(9) & 119(4) & $178(3)$ \\
\hline $\mathrm{C}(22)$ & 4953(3) & $7684(4)$ & 3338(2) & $83(1)$ \\
\hline$C(25)$ & $5595(5)$ & 5984(8) & 4073(3) & $141(2)$ \\
\hline
\end{tabular}




\begin{tabular}{lclcc}
$\mathrm{C}(26)$ & $5471(6)$ & $6626(10)$ & $4673(4)$ & $186(3)$ \\
$\mathrm{C}(28)$ & $-1478(4)$ & $7620(6)$ & $2532(3)$ & $111(2)$ \\
$\mathrm{C}(29 \mathrm{~A})$ & $-2090(30)$ & $6480(70)$ & $2550(20)$ & $137(13)$ \\
$\mathrm{C}(29 \mathrm{~B})$ & $-2016(17)$ & $6210(30)$ & $2620(15)$ & $102(6)$ \\
$\mathrm{C}(30)$ & $4602(4)$ & $7258(5)$ & $643(2)$ & $89(1)$ \\
$\mathrm{C}(33)$ & $4303(5)$ & $6911(8)$ & $-473(3)$ & $137(2)$ \\
$\mathrm{C}(34)$ & $3247(6)$ & $7460(7)$ & $-539(3)$ & $145(2)$ \\
$\mathrm{O}(35)$ & $7043(3)$ & $8893(4)$ & $871(2)$ & $123(1)$ \\
$\mathrm{O}(36)$ & $6748(3)$ & $6679(4)$ & $874(2)$ & $129(1)$ \\
$\mathrm{O}(17)$ & $525(2)$ & $8478(3)$ & $2007(1)$ & $103(1)$ \\
$\mathrm{O}(23)$ & $5805(3)$ & $8246(3)$ & $3382(1)$ & $105(1)$ \\
$\mathrm{O}(24 \mathrm{~A})$ & $4530(40)$ & $7040(70)$ & $3810(20)$ & $102(9)$ \\
$\mathrm{O}(24 \mathrm{~B})$ & $4763(10)$ & $6490(20)$ & $3632(8)$ & $92(4)$ \\
$\mathrm{O}(27)$ & $-394(2)$ & $7472(3)$ & $2791(2)$ & $100(1)$ \\
$\mathrm{O}(31)$ & $3965(3)$ & $6388(4)$ & $765(2)$ & $124(1)$ \\
$\mathrm{O}(32)$ & $4819(3)$ & $7666(4)$ & $63(2)$ & $113(1)$ \\
$\mathrm{S}(39)$ & $5139(1)$ & $7353(1)$ & $1893(1)$ & $93(1)$ \\
\hline
\end{tabular}

Table 3. Bond lengths $[\AA]$ and angles $\left[^{\circ}\right]$ for sp054.

\begin{tabular}{ll}
\hline $\mathrm{C}(21)-\mathrm{C}(13)$ & $1.528(6)$ \\
$\mathrm{C}(1)-\mathrm{C}(10)$ & $1.504(5)$ \\
$\mathrm{C}(1)-\mathrm{C}(2)$ & $1.556(5)$ \\
$\mathrm{C}(2)-\mathrm{C}(30)$ & $1.512(6)$ \\
$\mathrm{C}(2)-\mathrm{C}(3)$ & $1.519(6)$ \\
$\mathrm{C}(2)-\mathrm{S}(39)$ & $1.808(4)$ \\
$\mathrm{C}(3)-\mathrm{O}(35)$ & $1.196(5)$ \\
$\mathrm{C}(3)-\mathrm{O}(36)$ & $1.327(6)$ \\
$\mathrm{C}(7)-\mathrm{C}(8)$ & $1.329(6)$ \\
$\mathrm{C}(7)-\mathrm{O}(17)$ & $1.357(5)$ \\
$\mathrm{C}(8)-\mathrm{C}(9)$ & $1.470(5)$ \\
$\mathrm{C}(8)-\mathrm{C}(14)$ & $1.512(5)$ \\
$\mathrm{C}(9)-\mathrm{C}(10)$ & $1.341(4)$ \\
$\mathrm{C}(9)-\mathrm{C}(11)$ & $1.508(5)$ \\
$\mathrm{C}(10)-\mathrm{C}(18)$ & $1.498(5)$ \\
$\mathrm{C}(11)-\mathrm{C}(12)$ & $1.525(5)$ \\
$\mathrm{C}(11)-\mathrm{S}(39)$ & $1.828(4)$ \\
$\mathrm{C}(12)-\mathrm{C}(22)$ & $1.508(5)$ \\
$\mathrm{C}(12)-\mathrm{C}(13)$ & $1.550(5)$
\end{tabular}




\begin{tabular}{|c|c|}
\hline C(13)-C(14) & $1.539(5)$ \\
\hline C(14)-C(15) & $1.516(5)$ \\
\hline C(15)-C(16) & $1.503(6)$ \\
\hline $\mathrm{C}(16)-\mathrm{O}(27)$ & $1.392(5)$ \\
\hline $\mathrm{C}(16)-\mathrm{O}(17)$ & $1.436(5)$ \\
\hline C(37)-C(38) & $1.368(8)$ \\
\hline $\mathrm{C}(37)-\mathrm{O}(36)$ & $1.499(6)$ \\
\hline C(22)-O(23) & $1.192(5)$ \\
\hline $\mathrm{C}(22)-\mathrm{O}(24 \mathrm{~A})$ & $1.29(3)$ \\
\hline $\mathrm{C}(22)-\mathrm{O}(24 \mathrm{~B})$ & $1.363(13)$ \\
\hline C(25)-C(26) & $1.433(10)$ \\
\hline $\mathrm{C}(25)-\mathrm{O}(24 \mathrm{~B})$ & $1.471(10)$ \\
\hline $\mathrm{C}(25)-\mathrm{O}(24 \mathrm{~A})$ & $1.77(6)$ \\
\hline C(28)-C(29A) & $1.37(5)$ \\
\hline $\mathrm{C}(28)-\mathrm{O}(27)$ & $1.448(5)$ \\
\hline C(28)-C(29B) & $1.57(3)$ \\
\hline $\mathrm{C}(30)-\mathrm{O}(31)$ & $1.198(5)$ \\
\hline $\mathrm{C}(30)-\mathrm{O}(32)$ & $1.324(5)$ \\
\hline C(33)-C(34) & $1.416(8)$ \\
\hline $\mathrm{C}(33)-\mathrm{O}(32)$ & $1.503(6)$ \\
\hline$C(10)-C(1)-C(2)$ & 111.7(3) \\
\hline$C(30)-C(2)-C(3)$ & 108.1(3) \\
\hline$C(30)-C(2)-C(1)$ & $111.0(3)$ \\
\hline C(3)-C(2)-C(1) & $110.3(3)$ \\
\hline C(30)-C(2)-S(39) & $110.4(3)$ \\
\hline C(3)-C(2)-S(39) & 106.6(3) \\
\hline$C(1)-C(2)-S(39)$ & $110.4(3)$ \\
\hline $\mathrm{O}(35)-\mathrm{C}(3)-\mathrm{O}(36)$ & $124.7(4)$ \\
\hline $\mathrm{O}(35)-\mathrm{C}(3)-\mathrm{C}(2)$ & 124.1(5) \\
\hline $\mathrm{O}(36)-\mathrm{C}(3)-\mathrm{C}(2)$ & $111.2(4)$ \\
\hline $\mathrm{C}(8)-\mathrm{C}(7)-\mathrm{O}(17)$ & $127.8(4)$ \\
\hline C(7)-C(8)-C(9) & $120.6(3)$ \\
\hline C(7)-C(8)-C(14) & 118.1(3) \\
\hline C(9)-C(8)-C(14) & $120.9(3)$ \\
\hline$C(10)-C(9)-C(8)$ & $126.9(3)$ \\
\hline $\mathrm{C}(10)-\mathrm{C}(9)-\mathrm{C}(11)$ & $117.5(3)$ \\
\hline $\mathrm{C}(8)-\mathrm{C}(9)-\mathrm{C}(11)$ & $115.5(3)$ \\
\hline C(9)-C(10)-C(18) & 126.3(3) \\
\hline $\mathrm{C}(9)-\mathrm{C}(10)-\mathrm{C}(1)$ & $117.5(3)$ \\
\hline
\end{tabular}




\begin{tabular}{|c|c|}
\hline $\mathrm{C}(18)-\mathrm{C}(10)-\mathrm{C}(1)$ & 116.2(3) \\
\hline C(9)-C(11)-C(12) & $113.0(3)$ \\
\hline C(9)-C(11)-S(39) & $111.3(2)$ \\
\hline C(12)-C(11)-S(39) & $106.2(2)$ \\
\hline $\mathrm{C}(22)-\mathrm{C}(12)-\mathrm{C}(11)$ & $111.4(3)$ \\
\hline $\mathrm{C}(22)-\mathrm{C}(12)-\mathrm{C}(13)$ & $110.2(3)$ \\
\hline $\mathrm{C}(11)-\mathrm{C}(12)-\mathrm{C}(13)$ & 111.1(3) \\
\hline $\mathrm{C}(21)-\mathrm{C}(13)-\mathrm{C}(14)$ & $112.3(4)$ \\
\hline $\mathrm{C}(21)-\mathrm{C}(13)-\mathrm{C}(12)$ & $112.8(3)$ \\
\hline $\mathrm{C}(14)-\mathrm{C}(13)-\mathrm{C}(12)$ & 109.1(3) \\
\hline $\mathrm{C}(8)-\mathrm{C}(14)-\mathrm{C}(15)$ & $108.0(3)$ \\
\hline $\mathrm{C}(8)-\mathrm{C}(14)-\mathrm{C}(13)$ & $113.8(3)$ \\
\hline $\mathrm{C}(15)-\mathrm{C}(14)-\mathrm{C}(13)$ & $111.3(3)$ \\
\hline C(16)-C(15)-C(14) & $112.5(3)$ \\
\hline $\mathrm{O}(27)-\mathrm{C}(16)-\mathrm{O}(17)$ & $105.6(4)$ \\
\hline $\mathrm{O}(27)-\mathrm{C}(16)-\mathrm{C}(15)$ & $108.3(3)$ \\
\hline $\mathrm{O}(17)-\mathrm{C}(16)-\mathrm{C}(15)$ & $112.6(3)$ \\
\hline $\mathrm{C}(38)-\mathrm{C}(37)-\mathrm{O}(36)$ & $107.2(6)$ \\
\hline $\mathrm{O}(23)-\mathrm{C}(22)-\mathrm{O}(24 \mathrm{~A})$ & 122.1(13) \\
\hline $\mathrm{O}(23)-\mathrm{C}(22)-\mathrm{O}(24 \mathrm{~B})$ & $121.8(5)$ \\
\hline $\mathrm{O}(24 \mathrm{~A})-\mathrm{C}(22)-\mathrm{O}(24 \mathrm{~B})$ & $31(3)$ \\
\hline $\mathrm{O}(23)-\mathrm{C}(22)-\mathrm{C}(12)$ & $126.6(4)$ \\
\hline $\mathrm{O}(24 \mathrm{~A})-\mathrm{C}(22)-\mathrm{C}(12)$ & $105.7(17)$ \\
\hline $\mathrm{O}(24 \mathrm{~B})-\mathrm{C}(22)-\mathrm{C}(12)$ & $111.3(4)$ \\
\hline $\mathrm{C}(26)-\mathrm{C}(25)-\mathrm{O}(24 \mathrm{~B})$ & $109.6(13)$ \\
\hline $\mathrm{C}(26)-\mathrm{C}(25)-\mathrm{O}(24 \mathrm{~A})$ & $86(2)$ \\
\hline $\mathrm{O}(24 \mathrm{~B})-\mathrm{C}(25)-\mathrm{O}(24 \mathrm{~A})$ & $23.4(14)$ \\
\hline $\mathrm{C}(29 \mathrm{~A})-\mathrm{C}(28)-\mathrm{O}(27)$ & $114(3)$ \\
\hline$C(29 A)-C(28)-C(29 B)$ & $10(3)$ \\
\hline $\mathrm{O}(27)-\mathrm{C}(28)-\mathrm{C}(29 \mathrm{~B})$ & $104.7(11)$ \\
\hline $\mathrm{O}(31)-\mathrm{C}(30)-\mathrm{O}(32)$ & $123.9(4)$ \\
\hline $\mathrm{O}(31)-\mathrm{C}(30)-\mathrm{C}(2)$ & $126.3(4)$ \\
\hline $\mathrm{O}(32)-\mathrm{C}(30)-\mathrm{C}(2)$ & $109.8(4)$ \\
\hline $\mathrm{C}(34)-\mathrm{C}(33)-\mathrm{O}(32)$ & $105.4(5)$ \\
\hline $\mathrm{C}(3)-\mathrm{O}(36)-\mathrm{C}(37)$ & $118.8(5)$ \\
\hline $\mathrm{C}(7)-\mathrm{O}(17)-\mathrm{C}(16)$ & $117.6(3)$ \\
\hline $\mathrm{C}(22)-\mathrm{O}(24 \mathrm{~A})-\mathrm{C}(25)$ & $104(4)$ \\
\hline $\mathrm{C}(22)-\mathrm{O}(24 \mathrm{~B})-\mathrm{C}(25)$ & $117.9(7)$ \\
\hline $\mathrm{C}(16)-\mathrm{O}(27)-\mathrm{C}(28)$ & $114.9(4)$ \\
\hline $\mathrm{C}(30)-\mathrm{O}(32)-\mathrm{C}(33)$ & $117.6(4)$ \\
\hline
\end{tabular}


Symmetry transformations used to generate equivalent atoms:

Table 4. Anisotropic displacement parameters $\left(\AA^{2} \mathrm{x} 10^{3}\right)$ for sp054. The anisotropic displacement factor exponent takes the form: $-2 \pi^{2}\left[h^{2} a^{* 2} U^{11}+\ldots+2 h k a^{*} b^{*} U^{12}\right]$

\begin{tabular}{|c|c|c|c|c|c|c|}
\hline & $\mathrm{U}^{11}$ & $\mathrm{U}^{22}$ & $\mathrm{U}^{33}$ & $\mathrm{U}^{23}$ & $\mathrm{U}^{13}$ & $\mathrm{U}^{12}$ \\
\hline $\mathrm{C}(21)$ & $120(4)$ & $95(3)$ & 114(3) & $-32(3)$ & $1(3)$ & $-15(3)$ \\
\hline $\mathrm{C}(1)$ & $77(2)$ & $82(3)$ & $74(2)$ & $7(2)$ & $7(2)$ & $-3(2)$ \\
\hline $\mathrm{C}(2)$ & $73(2)$ & $80(2)$ & $81(2)$ & $-2(2)$ & $8(2)$ & $2(2)$ \\
\hline C(3) & 82(3) & $109(4)$ & $98(3)$ & $-9(3)$ & $9(2)$ & $0(3)$ \\
\hline$C(7)$ & $82(3)$ & $92(3)$ & $83(3)$ & $15(2)$ & $4(2)$ & $-5(2)$ \\
\hline $\mathrm{C}(8)$ & $78(2)$ & $69(2)$ & $67(2)$ & $8(2)$ & $4(2)$ & $4(2)$ \\
\hline C(9) & $74(2)$ & $67(2)$ & $66(2)$ & $5(2)$ & $-1(2)$ & $4(2)$ \\
\hline$C(10)$ & $81(2)$ & $71(2)$ & $66(2)$ & $4(2)$ & $2(2)$ & $1(2)$ \\
\hline $\mathrm{C}(11)$ & $73(2)$ & $77(2)$ & $66(2)$ & $6(2)$ & $1(2)$ & $-4(2)$ \\
\hline $\mathrm{C}(12)$ & $76(2)$ & $75(2)$ & $69(2)$ & $9(2)$ & $-4(2)$ & $-5(2)$ \\
\hline C(13) & 88(3) & $84(3)$ & $69(2)$ & $1(2)$ & $5(2)$ & $-5(2)$ \\
\hline$C(14)$ & $81(2)$ & $77(2)$ & $77(2)$ & $2(2)$ & $7(2)$ & $3(2)$ \\
\hline $\mathrm{C}(15)$ & $79(2)$ & $105(3)$ & $80(2)$ & $9(2)$ & $12(2)$ & $-4(2)$ \\
\hline$C(16)$ & $80(2)$ & $93(3)$ & 93(3) & $9(2)$ & $7(2)$ & $4(2)$ \\
\hline C(18) & $88(3)$ & 91(3) & $78(2)$ & $17(2)$ & $-1(2)$ & $9(2)$ \\
\hline C(37) & 111(4) & 213(8) & $157(6)$ & $-55(5)$ & $15(4)$ & $20(5)$ \\
\hline C(38) & $178(7)$ & 204(8) & 153(6) & $-33(5)$ & $65(5)$ & $-19(6)$ \\
\hline $\mathrm{C}(22)$ & $81(3)$ & 93(3) & $74(2)$ & $14(2)$ & $-8(2)$ & $-7(2)$ \\
\hline$C(25)$ & $110(4)$ & 179(6) & 132(5) & $47(5)$ & $-40(3)$ & $-20(4)$ \\
\hline C(26) & $157(6)$ & $244(10)$ & $157(7)$ & $42(7)$ & $13(5)$ & $8(7)$ \\
\hline$C(28)$ & 72(3) & $136(5)$ & $125(4)$ & $26(3)$ & $-2(3)$ & $12(3)$ \\
\hline$C(29 A)$ & 117(19) & $180(30)$ & $115(13)$ & $38(18)$ & 23(16) & $30(17)$ \\
\hline $\mathrm{C}(29 \mathrm{~B})$ & $65(8)$ & $88(13)$ & 152(17) & $10(8)$ & $-40(11)$ & $-6(6)$ \\
\hline C(30) & $97(3)$ & $92(3)$ & $77(2)$ & $-3(2)$ & $8(2)$ & $-10(2)$ \\
\hline C(33) & $119(4)$ & 192(6) & 101(4) & $-13(4)$ & $7(3)$ & $-16(4)$ \\
\hline$C(34)$ & $139(5)$ & $147(6)$ & 149(6) & $11(4)$ & $-29(4)$ & $-14(4)$ \\
\hline $\mathrm{O}(35)$ & $84(2)$ & $130(3)$ & 154(3) & $-7(2)$ & $19(2)$ & $-10(2)$ \\
\hline $\mathrm{O}(36)$ & $94(2)$ & 126(3) & 166(3) & $-37(3)$ & $24(2)$ & $20(2)$ \\
\hline $\mathrm{O}(17)$ & $78(2)$ & 136(3) & $94(2)$ & $25(2)$ & $2(1)$ & $-9(2)$ \\
\hline $\mathrm{O}(23)$ & $94(2)$ & $118(2)$ & $103(2)$ & $22(2)$ & $-22(2)$ & $-23(2)$ \\
\hline
\end{tabular}




\begin{tabular}{lcccccc}
$\mathrm{O}(24 \mathrm{~A})$ & $107(13)$ & $85(19)$ & $114(15)$ & $33(14)$ & $-15(11)$ & $8(14)$ \\
$\mathrm{O}(24 \mathrm{~B})$ & $86(4)$ & $81(7)$ & $110(5)$ & $37(5)$ & $-15(3)$ & $2(4)$ \\
$\mathrm{O}(27)$ & $70(2)$ & $116(2)$ & $115(2)$ & $28(2)$ & $3(2)$ & $-1(2)$ \\
$\mathrm{O}(31)$ & $140(3)$ & $126(3)$ & $106(2)$ & $2(2)$ & $0(2)$ & $-49(2)$ \\
$\mathrm{O}(32)$ & $125(3)$ & $136(3)$ & $79(2)$ & $-7(2)$ & $-1(2)$ & $-35(2)$ \\
$\mathrm{S}(39)$ & $106(1)$ & $91(1)$ & $83(1)$ & $10(1)$ & $7(1)$ & $25(1)$ \\
\hline
\end{tabular}

Table 5. Hydrogen coordinates ( $\left.\mathrm{x} 10^{4}\right)$ and isotropic displacement parameters $\left(\AA^{2} \times 10^{3}\right)$ for sp054.

\begin{tabular}{|c|c|c|c|c|}
\hline & $\mathrm{x}$ & $\mathrm{y}$ & $\mathrm{z}$ & $\mathrm{U}(\mathrm{eq})$ \\
\hline $\mathrm{H}(01 \mathrm{~A})$ & 3983 & 10870 & 3078 & 165 \\
\hline $\mathrm{H}(01 \mathrm{~B})$ & 4468 & 10182 & 3681 & 165 \\
\hline $\mathrm{H}(01 \mathrm{C})$ & 3336 & 10900 & 3712 & 165 \\
\hline $\mathrm{H}(7)$ & $1640(30)$ & $8510(40)$ & $1390(20)$ & $85(12)$ \\
\hline $\mathrm{H}(00 \mathrm{~A})$ & 5373 & 10070 & 1392 & 93 \\
\hline $\mathrm{H}(00 \mathrm{~B})$ & 4969 & 9926 & 693 & 93 \\
\hline $\mathrm{H}(013)$ & 4909 & 9418 & 2396 & 86 \\
\hline $\mathrm{H}(004)$ & 3595 & 7266 & 2820 & 88 \\
\hline $\mathrm{H}(008)$ & 3024 & 8621 & 3663 & 96 \\
\hline $\mathrm{H}(009)$ & 1980 & 10220 & 2941 & 94 \\
\hline $\mathrm{H}(01 \mathrm{D})$ & 1555 & 7443 & 3036 & 105 \\
\hline $\mathrm{H}(01 \mathrm{E})$ & 1132 & 8537 & 3505 & 105 \\
\hline $\mathrm{H}(014)$ & -53 & 9392 & 2773 & 106 \\
\hline $\mathrm{H}(01 \mathrm{~F})$ & 2362 & 10726 & 1101 & 129 \\
\hline $\mathrm{H}(01 \mathrm{G})$ & 2751 & 9767 & 561 & 129 \\
\hline $\mathrm{H}(01 \mathrm{H})$ & 3350 & 11134 & 679 & 129 \\
\hline $\mathrm{H}(02 \mathrm{~A})$ & 8372 & 7129 & 859 & 193 \\
\hline $\mathrm{H}(02 \mathrm{~B})$ & 8150 & 5600 & 996 & 193 \\
\hline $\mathrm{H}(02 \mathrm{C})$ & 7404 & 5537 & -11 & 267 \\
\hline $\mathrm{H}(02 \mathrm{D})$ & 8672 & 5624 & 42 & 267 \\
\hline $\mathrm{H}(02 \mathrm{E})$ & 7993 & 6913 & -116 & 267 \\
\hline $\mathrm{H}(02 \mathrm{I})$ & 5417 & 5036 & 4072 & 169 \\
\hline $\mathrm{H}(02 \mathrm{~J})$ & 6289 & 6150 & 3873 & 169 \\
\hline $\mathrm{H}(02 \mathrm{~F})$ & 5607 & 7570 & 4630 & 279 \\
\hline $\mathrm{H}(02 \mathrm{G})$ & 5978 & 6247 & 4968 & 279 \\
\hline $\mathrm{H}(02 \mathrm{H})$ & 4744 & 6489 & 4821 & 279 \\
\hline
\end{tabular}




\begin{tabular}{lllll}
$\mathrm{H}(01 \mathrm{I})$ & -1853 & 8317 & 2765 & 133 \\
$\mathrm{H}(01 \mathrm{~J})$ & -1421 & 7917 & 2098 & 133 \\
$\mathrm{H}(29 \mathrm{~A})$ & -1763 & 5805 & 2286 & 206 \\
$\mathrm{H}(29 \mathrm{~B})$ & -2810 & 6670 & 2394 & 206 \\
$\mathrm{H}(29 \mathrm{C})$ & -2131 & 6154 & 2973 & 206 \\
$\mathrm{H}(29 \mathrm{D})$ & -1685 & 5575 & 2339 & 153 \\
$\mathrm{H}(29 \mathrm{E})$ & -2779 & 6267 & 2528 & 153 \\
$\mathrm{H}(29 \mathrm{~F})$ & -1914 & 5913 & 3047 & 153 \\
$\mathrm{H}(01 \mathrm{~K})$ & 4714 & 7033 & -858 & 165 \\
$\mathrm{H}(01 \mathrm{~L})$ & 4265 & 5959 & -379 & 165 \\
$\mathrm{H}(01 \mathrm{M})$ & 2837 & 7282 & -164 & 218 \\
$\mathrm{H}(01 \mathrm{~N})$ & 2888 & 7057 & -895 & 218 \\
$\mathrm{H}(01 \mathrm{O})$ & 3298 & 8412 & -602 & 218 \\
\hline
\end{tabular}

Table 6. Torsion angles [ $\left.{ }^{\circ}\right]$ for sp054.

\begin{tabular}{lc}
\hline$C(10)-C(1)-C(2)-C(30)$ & $63.8(4)$ \\
$C(10)-C(1)-C(2)-C(3)$ & $-176.4(3)$ \\
$C(10)-C(1)-C(2)-S(39)$ & $-58.9(4)$ \\
$C(30)-C(2)-C(3)-O(35)$ & $128.8(5)$ \\
$C(1)-C(2)-C(3)-O(35)$ & $7.3(6)$ \\
S(39)-C(2)-C(3)-O(35) & $-112.6(5)$ \\
$C(30)-C(2)-C(3)-O(36)$ & $-52.8(5)$ \\
$C(1)-C(2)-C(3)-O(36)$ & $-174.3(4)$ \\
$S(39)-C(2)-C(3)-O(36)$ & $65.8(4)$ \\
$O(17)-C(7)-C(8)-C(9)$ & $175.8(4)$ \\
$O(17)-C(7)-C(8)-C(14)$ & $3.1(6)$ \\
$C(7)-C(8)-C(9)-C(10)$ & $41.6(6)$ \\
$C(14)-C(8)-C(9)-C(10)$ & $-145.9(4)$ \\
$C(7)-C(8)-C(9)-C(11)$ & $-136.2(4)$ \\
$C(14)-C(8)-C(9)-C(11)$ & $36.3(5)$ \\
$C(8)-C(9)-C(10)-C(18)$ & $7.4(6)$ \\
$C(11)-C(9)-C(10)-C(18)$ & $-174.8(4)$ \\
$C(8)-C(9)-C(10)-C(1)$ & $-171.9(3)$ \\
$C(11)-C(9)-C(10)-C(1)$ & $5.8(5)$ \\
$C(2)-C(1)-C(10)-C(9)$ & $57.0(4)$ \\
$C(2)-C(1)-C(10)-C(18)$ & $-122.4(4)$ \\
$C(10)-C(9)-C(11)-C(12)$ & $-176.5(3)$ \\
$C(8)-C(9)-C(11)-C(12)$ & $1.5(5)$ \\
& \\
& \\
$C$ &
\end{tabular}




\begin{tabular}{|c|c|}
\hline C(10)-C(9)-C(11)-S(39) & $-57.1(4)$ \\
\hline C(8)-C(9)-C(11)-S(39) & $120.9(3)$ \\
\hline $\mathrm{C}(9)-\mathrm{C}(11)-\mathrm{C}(12)-\mathrm{C}(22)$ & $-173.9(3)$ \\
\hline S(39)-C(11)-C(12)-C(22) & $63.8(4)$ \\
\hline C(9)-C(11)-C(12)-C(13) & $-50.7(4)$ \\
\hline S(39)-C(11)-C(12)-C(13) & $-172.9(2)$ \\
\hline C(22)-C(12)-C(13)-C(21) & $62.2(4)$ \\
\hline $\mathrm{C}(11)-\mathrm{C}(12)-\mathrm{C}(13)-\mathrm{C}(21)$ & $-61.7(4)$ \\
\hline C(22)-C(12)-C(13)-C(14) & $-172.3(3)$ \\
\hline C(11)-C(12)-C(13)-C(14) & $63.7(4)$ \\
\hline C(7)-C(8)-C(14)-C(15) & 26.7(5) \\
\hline C(9)-C(8)-C(14)-C(15) & $-145.9(3)$ \\
\hline C(7)-C(8)-C(14)-C(13) & $150.8(4)$ \\
\hline C(9)-C(8)-C(14)-C(13) & $-21.9(5)$ \\
\hline C(21)-C(13)-C(14)-C(8) & $98.7(4)$ \\
\hline $\mathrm{C}(12)-\mathrm{C}(13)-\mathrm{C}(14)-\mathrm{C}(8)$ & $-27.1(4)$ \\
\hline C(21)-C(13)-C(14)-C(15) & $-139.0(4)$ \\
\hline C(12)-C(13)-C(14)-C(15) & $95.2(4)$ \\
\hline C(8)-C(14)-C(15)-C(16) & $-52.6(5)$ \\
\hline C(13)-C(14)-C(15)-C(16) & $-178.2(3)$ \\
\hline $\mathrm{C}(14)-\mathrm{C}(15)-\mathrm{C}(16)-\mathrm{O}(27)$ & $167.4(3)$ \\
\hline $\mathrm{C}(14)-\mathrm{C}(15)-\mathrm{C}(16)-\mathrm{O}(17)$ & $51.1(5)$ \\
\hline $\mathrm{C}(11)-\mathrm{C}(12)-\mathrm{C}(22)-\mathrm{O}(23)$ & $34.2(6)$ \\
\hline C(13)-C(12)-C(22)-O(23) & $-89.5(5)$ \\
\hline $\mathrm{C}(11)-\mathrm{C}(12)-\mathrm{C}(22)-\mathrm{O}(24 \mathrm{~A})$ & $-172(4)$ \\
\hline $\mathrm{C}(13)-\mathrm{C}(12)-\mathrm{C}(22)-\mathrm{O}(24 \mathrm{~A})$ & $64(4)$ \\
\hline $\mathrm{C}(11)-\mathrm{C}(12)-\mathrm{C}(22)-\mathrm{O}(24 \mathrm{~B})$ & $-139.7(13)$ \\
\hline $\mathrm{C}(13)-\mathrm{C}(12)-\mathrm{C}(22)-\mathrm{O}(24 \mathrm{~B})$ & 96.5(13) \\
\hline $\mathrm{C}(3)-\mathrm{C}(2)-\mathrm{C}(30)-\mathrm{O}(31)$ & $126.0(5)$ \\
\hline $\mathrm{C}(1)-\mathrm{C}(2)-\mathrm{C}(30)-\mathrm{O}(31)$ & $-112.9(5)$ \\
\hline $\mathrm{S}(39)-\mathrm{C}(2)-\mathrm{C}(30)-\mathrm{O}(31)$ & $9.8(6)$ \\
\hline C(3)-C(2)-C(30)-O(32) & $-54.9(5)$ \\
\hline C(1)-C(2)-C(30)-O(32) & $66.2(4)$ \\
\hline S(39)-C(2)-C(30)-O(32) & $-171.1(3)$ \\
\hline $\mathrm{O}(35)-\mathrm{C}(3)-\mathrm{O}(36)-\mathrm{C}(37)$ & $3.1(8)$ \\
\hline $\mathrm{C}(2)-\mathrm{C}(3)-\mathrm{O}(36)-\mathrm{C}(37)$ & $-175.3(4)$ \\
\hline C(38)-C(37)-O(36)-C(3) & $-101.5(7)$ \\
\hline C(8)-C(7)-O(17)-C(16) & $-7.0(6)$ \\
\hline $\mathrm{O}(27)-\mathrm{C}(16)-\mathrm{O}(17)-\mathrm{C}(7)$ & $-138.6(4)$ \\
\hline $\mathrm{C}(15)-\mathrm{C}(16)-\mathrm{O}(17)-\mathrm{C}(7)$ & $-20.6(5)$ \\
\hline
\end{tabular}




$\begin{array}{lc}\mathrm{O}(23)-\mathrm{C}(22)-\mathrm{O}(24 \mathrm{~A})-\mathrm{C}(25) & -50(5) \\ \mathrm{O}(24 \mathrm{~B})-\mathrm{C}(22)-\mathrm{O}(24 \mathrm{~A})-\mathrm{C}(25) & 49(4) \\ \mathrm{C}(12)-\mathrm{C}(22)-\mathrm{O}(24 \mathrm{~A})-\mathrm{C}(25) & 155(2) \\ \mathrm{C}(26)-\mathrm{C}(25)-\mathrm{O}(24 \mathrm{~A})-\mathrm{C}(22) & 122(4) \\ \mathrm{O}(24 \mathrm{~B})-\mathrm{C}(25)-\mathrm{O}(24 \mathrm{~A})-\mathrm{C}(22) & -66(4) \\ \mathrm{O}(23)-\mathrm{C}(22)-\mathrm{O}(24 \mathrm{~B})-\mathrm{C}(25) & 8(3) \\ \mathrm{O}(24 \mathrm{~A})-\mathrm{C}(22)-\mathrm{O}(24 \mathrm{~B})-\mathrm{C}(25) & -93(4) \\ \mathrm{C}(12)-\mathrm{C}(22)-\mathrm{O}(24 \mathrm{~B})-\mathrm{C}(25) & -178.2(14) \\ \mathrm{C}(26)-\mathrm{C}(25)-\mathrm{O}(24 \mathrm{~B})-\mathrm{C}(22) & 81.6(19) \\ \mathrm{O}(24 \mathrm{~A})-\mathrm{C}(25)-\mathrm{O}(24 \mathrm{~B})-\mathrm{C}(22) & 72(3) \\ \mathrm{O}(17)-\mathrm{C}(16)-\mathrm{O}(27)-\mathrm{C}(28) & -69.9(5) \\ \mathrm{C}(15)-\mathrm{C}(16)-\mathrm{O}(27)-\mathrm{C}(28) & 169.3(4) \\ \mathrm{C}(29 \mathrm{~A})-\mathrm{C}(28)-\mathrm{O}(27)-\mathrm{C}(16) & 168.2(15) \\ \mathrm{C}(29 \mathrm{~B})-\mathrm{C}(28)-\mathrm{O}(27)-\mathrm{C}(16) & 169.7(11) \\ \mathrm{O}(31)-\mathrm{C}(30)-\mathrm{O}(32)-\mathrm{C}(33) & -5.3(7) \\ \mathrm{C}(2)-\mathrm{C}(30)-\mathrm{O}(32)-\mathrm{C}(33) & 175.5(4) \\ \mathrm{C}(34)-\mathrm{C}(33)-\mathrm{O}(32)-\mathrm{C}(30) & 83.1(6) \\ \mathrm{C}(30)-\mathrm{C}(2)-\mathrm{S}(39)-\mathrm{C}(11) & -113.4(3) \\ \mathrm{C}(3)-\mathrm{C}(2)-\mathrm{S}(39)-\mathrm{C}(11) & 129.5(3) \\ \mathrm{C}(1)-\mathrm{C}(2)-\mathrm{S}(39)-\mathrm{C}(11) & 9.7(3) \\ \mathrm{C}(9)-\mathrm{C}(11)-\mathrm{S}(39)-\mathrm{C}(2) & 43.6(3) \\ \mathrm{C}(12)-\mathrm{C}(11)-\mathrm{S}(39)-\mathrm{C}(2) & 166.9(2)\end{array}$

Symmetry transformations used to generate equivalent atoms: 


\section{Triester 20a (CCDC 600926)}

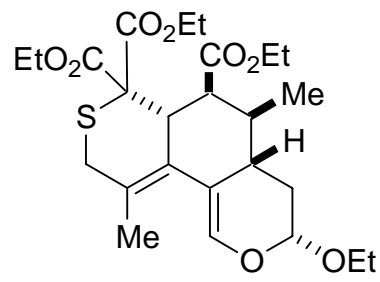

Experimental : The crystal was grown by cooling of a saturated solution using ethanol at room temperature. One single crystal was mounted using a glass fiber on the goniometer. Data were collected on an Enraf-Nonius CAD-4 automatic diffractometer at the Université de Sherbrooke using $\omega$ scans at 293(2) K. The DIFRAC ${ }^{(1)}$ program was used for centering, indexing, and data collection. Two standard reflections were measured every 200 reflections, $11.3 \%$ intensity decay was observed during data collection. The data were reduced and corrected for absorption by empirical methods based on Psi Scan measurements with the NRCVAX ${ }^{(2)}$ programs. They were solved using SHELXS- $97^{(3)}$ and refined by full-matrix least squares on $\mathrm{F}^{2}$ with SHELXL-97 ${ }^{(4)}$. The non-hydrogen atoms were refined anisotropically. The hydrogen atoms were placed at idealized calculated geometric position and refined isotropically using a riding model.

(1) H.D. Flack, E. Blanc and D. Schwarzenbach (1992), J. Appl. Cryst., 25, 455-459.

(2) E.J. Gabe, Y. Le Page, J.-P. Charland, F.L. Lee, and P.S. White, (1989) J. Appl. Cryst., 22, 384-387.

(3) G. M. Sheldrick, SHELXS-97, G.M. Sheldrick, University of Göttingen, Germany, 1997, Release 97-2.

(4) G. M. Sheldrick, SHELXL-97, G.M. Sheldrick, University of Göttingen, Germany, 1997, Release 97-2. 


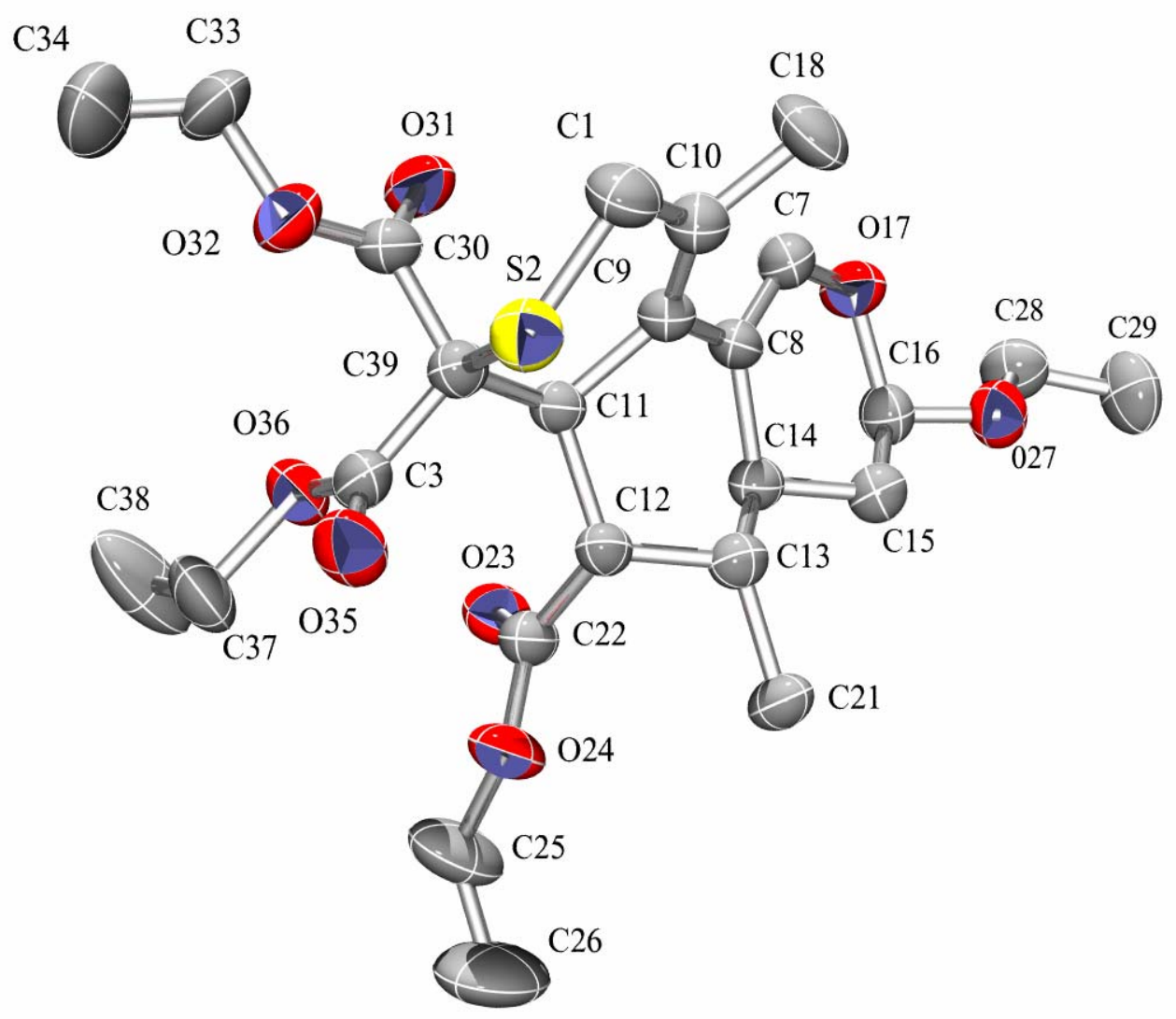

Table 1. Crystal data and structure refinement for sp3-053tpièce.

Identification code

Empirical formula

Formula weight

Temperature

Wavelength

Crystal system

Space group

Unit cell dimensions

Volume

Z

Density (calculated)

Absorption coefficient

$\mathrm{F}(000)$ sp3053tpi

C25 H36 O8 S

496.60

293(2) K

$1.54176 \AA$

Monoclinic

P121/n1

$\begin{array}{ll}\mathrm{a}=9.1859(9) \AA & \alpha=90.000(12)^{\circ} . \\ \mathrm{b}=10.0904(18) \AA & \beta=94.256(9)^{\circ} . \\ \mathrm{c}=29.133(4) \AA & \gamma=90.000(11)^{\circ} .\end{array}$

2692.8(6) $\AA^{3}$

4

$1.225 \mathrm{Mg} / \mathrm{m}^{3}$

$1.437 \mathrm{~mm}^{-1}$

1064 
Crystal size

Theta range for data collection

Index ranges

Reflections collected

Independent reflections

Completeness to theta $=69.91^{\circ}$

Absorption correction

Refinement method

Data / restraints / parameters

Goodness-of-fit on $\mathrm{F}^{2}$

Final R indices [I>2sigma(I)]

$\mathrm{R}$ indices (all data)

Extinction coefficient

Largest diff. peak and hole
$0.4 \times 0.4 \times 0.4 \mathrm{~mm}^{3}$

3.04 to $69.91^{\circ}$.

$-11<=\mathrm{h}<=11,0<=\mathrm{k}<=12,0<=\mathrm{l}<=35$

5016

$5016[$ R(int) $=0.0000]$

$97.9 \%$

Empirical

Full-matrix least-squares on $\mathrm{F}^{2}$

5016 / 0 / 326

0.997

$\mathrm{R} 1=0.0684, \mathrm{wR} 2=0.1771$

$\mathrm{R} 1=0.1385, \mathrm{wR} 2=0.2125$

0.0059(5)

0.232 and -0.208 e. $\AA^{-3}$

Table 2. Atomic coordinates ( $\left.\mathrm{x} 10^{4}\right)$ and equivalent isotropic displacement parameters $\left(\AA^{2} \mathrm{x} 10^{3}\right)$ for sp3-053tpièce. $U(\mathrm{eq})$ is defined as one third of the trace of the orthogonalized $\mathrm{U}^{\mathrm{ij}}$ tensor.

\begin{tabular}{|c|c|c|c|c|}
\hline & $\mathrm{x}$ & $\mathrm{y}$ & $\mathrm{z}$ & $\mathrm{U}(\mathrm{eq})$ \\
\hline $\mathrm{O}(24)$ & 9534(3) & 3542(3) & $2156(1)$ & $113(1)$ \\
\hline$C(25)$ & 10829(8) & 2703(6) & $2287(2)$ & 166(3) \\
\hline$C(26)$ & 11542(9) & $3170(9)$ & 2687(3) & $240(4)$ \\
\hline C(38) & $7747(11)$ & $-495(7)$ & $1762(2)$ & $218(4)$ \\
\hline $\mathrm{C}(1)$ & $3875(5)$ & $5639(5)$ & 1152(2) & 106(1) \\
\hline $\mathrm{C}(3)$ & $6013(5)$ & 2618(4) & $1820(2)$ & $90(1)$ \\
\hline$C(7)$ & $7664(5)$ & $6159(4)$ & 223(2) & 93(1) \\
\hline$C(8)$ & 7702(4) & $5795(4)$ & 661(1) & $79(1)$ \\
\hline C(9) & $6428(4)$ & $5400(4)$ & $906(1)$ & $80(1)$ \\
\hline$C(10)$ & $5125(5)$ & $6028(4)$ & $874(1)$ & $94(1)$ \\
\hline $\mathrm{C}(11)$ & $6842(4)$ & $4244(3)$ & 1231(1) & $76(1)$ \\
\hline $\mathrm{C}(12)$ & $8017(4)$ & 4698(3) & $1607(1)$ & $73(1)$ \\
\hline$C(13)$ & $8821(4)$ & 5962(3) & 1458(1) & $79(1)$ \\
\hline$C(14)$ & $9144(4)$ & $5855(4)$ & 951(1) & $77(1)$ \\
\hline$C(15)$ & $9977(4)$ & $7024(4)$ & 779(1) & $89(1)$ \\
\hline$C(16)$ & 10196(4) & $6875(4)$ & $276(1)$ & $90(1)$ \\
\hline $\mathrm{C}(18)$ & $4769(5)$ & 7181(5) & $554(2)$ & $131(2)$ \\
\hline$C(21)$ & 10184(4) & $6297(4)$ & $1775(1)$ & $94(1)$ \\
\hline $\mathrm{C}(22)$ & $9144(4)$ & $3624(4)$ & $1710(1)$ & $86(1)$ \\
\hline
\end{tabular}




\begin{tabular}{lrrrr}
$\mathrm{C}(28)$ & $11353(5)$ & $7986(5)$ & $-319(1)$ & $107(1)$ \\
$\mathrm{C}(29)$ & $12085(7)$ & $9267(5)$ & $-394(2)$ & $141(2)$ \\
$\mathrm{C}(30)$ & $4661(5)$ & $2774(4)$ & $1051(2)$ & $94(1)$ \\
$\mathrm{C}(33)$ & $2770(6)$ & $1205(6)$ & $899(2)$ & $148(2)$ \\
$\mathrm{C}(34)$ & $2086(9)$ & $221(6)$ & $1131(3)$ & $193(3)$ \\
$\mathrm{C}(37)$ & $7171(6)$ & $573(5)$ & $2000(2)$ & $129(2)$ \\
$\mathrm{C}(39)$ & $5536(4)$ & $3560(4)$ & $1431(1)$ & $81(1)$ \\
$\mathrm{O}(17)$ & $8838(3)$ & $6621(3)$ & $1(1)$ & $106(1)$ \\
$\mathrm{O}(23)$ & $9657(3)$ & $2949(3)$ & $1427(1)$ & $106(1)$ \\
$\mathrm{O}(31)$ & $4880(3)$ & $2796(3)$ & $653(1)$ & $111(1)$ \\
$\mathrm{O}(32)$ & $3647(3)$ & $2029(3)$ & $1230(1)$ & $115(1)$ \\
$\mathrm{O}(35)$ & $5814(4)$ & $2809(3)$ & $2217(1)$ & $118(1)$ \\
$\mathrm{O}(36)$ & $6674(3)$ & $1573(3)$ & $1659(1)$ & $102(1)$ \\
$\mathrm{O}(27)$ & $10775(3)$ & $8053(3)$ & $126(1)$ & $100(1)$ \\
$\mathrm{S}(2)$ & $4359(1)$ & $4780(1)$ & $1676(1)$ & $102(1)$ \\
\hline
\end{tabular}

Table 3. Bond lengths $[\AA]$ and angles $\left[{ }^{\circ}\right]$ for sp3-053tpièce.

\begin{tabular}{ll}
\hline $\mathrm{O}(24)-\mathrm{C}(22)$ & $1.323(4)$ \\
$\mathrm{O}(24)-\mathrm{C}(25)$ & $1.487(6)$ \\
$\mathrm{C}(25)-\mathrm{C}(26)$ & $1.377(8)$ \\
$\mathrm{C}(38)-\mathrm{C}(37)$ & $1.406(7)$ \\
$\mathrm{C}(1)-\mathrm{C}(10)$ & $1.504(6)$ \\
$\mathrm{C}(1)-\mathrm{S}(2)$ & $1.784(4)$ \\
$\mathrm{C}(3)-\mathrm{O}(35)$ & $1.199(5)$ \\
$\mathrm{C}(3)-\mathrm{O}(36)$ & $1.321(5)$ \\
$\mathrm{C}(3)-\mathrm{C}(39)$ & $1.520(5)$ \\
$\mathrm{C}(7)-\mathrm{C}(8)$ & $1.326(5)$ \\
$\mathrm{C}(7)-\mathrm{O}(17)$ & $1.380(5)$ \\
$\mathrm{C}(8)-\mathrm{C}(9)$ & $1.470(5)$ \\
$\mathrm{C}(8)-\mathrm{C}(14)$ & $1.519(4)$ \\
$\mathrm{C}(9)-\mathrm{C}(10)$ & $1.352(5)$ \\
$\mathrm{C}(9)-\mathrm{C}(11)$ & $1.532(5)$ \\
$\mathrm{C}(10)-\mathrm{C}(18)$ & $1.511(6)$ \\
$\mathrm{C}(11)-\mathrm{C}(39)$ & $1.536(5)$ \\
$\mathrm{C}(11)-\mathrm{C}(12)$ & $1.548(4)$ \\
$\mathrm{C}(12)-\mathrm{C}(22)$ & $1.514(5)$ \\
$\mathrm{C}(12)-\mathrm{C}(13)$ & $1.552(5)$ \\
$\mathrm{C}(13)-\mathrm{C}(14)$ & $1.531(5)$ \\
\end{tabular}




\begin{tabular}{|c|c|}
\hline C(13)-C(21) & $1.537(5)$ \\
\hline$C(14)-C(15)$ & $1.512(5)$ \\
\hline C(15)-C(16) & $1.502(5)$ \\
\hline $\mathrm{C}(16)-\mathrm{O}(27)$ & $1.386(4)$ \\
\hline $\mathrm{C}(16)-\mathrm{O}(17)$ & $1.454(4)$ \\
\hline $\mathrm{C}(22)-\mathrm{O}(23)$ & $1.194(4)$ \\
\hline $\mathrm{C}(28)-\mathrm{O}(27)$ & $1.439(5)$ \\
\hline C(28)-C(29) & $1.480(7)$ \\
\hline $\mathrm{C}(30)-\mathrm{O}(31)$ & 1.191(5) \\
\hline $\mathrm{C}(30)-\mathrm{O}(32)$ & $1.332(5)$ \\
\hline C(30)-C(39) & $1.538(5)$ \\
\hline C(33)-C(34) & $1.379(7)$ \\
\hline $\mathrm{C}(33)-\mathrm{O}(32)$ & $1.468(5)$ \\
\hline $\mathrm{C}(37)-\mathrm{O}(36)$ & $1.465(5)$ \\
\hline C(39)-S(2) & $1.820(4)$ \\
\hline C(22)-O(24)-C(25) & $116.2(4)$ \\
\hline C(26)-C(25)-O(24) & $110.1(6)$ \\
\hline$C(10)-C(1)-S(2)$ & $115.8(3)$ \\
\hline $\mathrm{O}(35)-\mathrm{C}(3)-\mathrm{O}(36)$ & $125.4(4)$ \\
\hline O(35)-C(3)-C(39) & $124.3(4)$ \\
\hline $\mathrm{O}(36)-\mathrm{C}(3)-\mathrm{C}(39)$ & $110.3(3)$ \\
\hline $\mathrm{C}(8)-\mathrm{C}(7)-\mathrm{O}(17)$ & $125.5(4)$ \\
\hline C(7)-C(8)-C(9) & $125.2(3)$ \\
\hline C(7)-C(8)-C(14) & $118.9(4)$ \\
\hline C(9)-C(8)-C(14) & $115.7(3)$ \\
\hline C(10)-C(9)-C(8) & $124.9(3)$ \\
\hline $\mathrm{C}(10)-\mathrm{C}(9)-\mathrm{C}(11)$ & $125.3(4)$ \\
\hline C(8)-C(9)-C(11) & $109.6(3)$ \\
\hline $\mathrm{C}(9)-\mathrm{C}(10)-\mathrm{C}(1)$ & $123.2(4)$ \\
\hline $\mathrm{C}(9)-\mathrm{C}(10)-\mathrm{C}(18)$ & $123.6(4)$ \\
\hline $\mathrm{C}(1)-\mathrm{C}(10)-\mathrm{C}(18)$ & $113.2(4)$ \\
\hline C(9)-C(11)-C(39) & $114.3(3)$ \\
\hline $\mathrm{C}(9)-\mathrm{C}(11)-\mathrm{C}(12)$ & 109.8(3) \\
\hline C(39)-C(11)-C(12) & $112.8(3)$ \\
\hline $\mathrm{C}(22)-\mathrm{C}(12)-\mathrm{C}(11)$ & 111.1(3) \\
\hline $\mathrm{C}(22)-\mathrm{C}(12)-\mathrm{C}(13)$ & $108.2(3)$ \\
\hline $\mathrm{C}(11)-\mathrm{C}(12)-\mathrm{C}(13)$ & $111.6(3)$ \\
\hline$C(14)-C(13)-C(21)$ & $112.8(3)$ \\
\hline C(14)-C(13)-C(12) & $109.9(3)$ \\
\hline
\end{tabular}




\begin{tabular}{|c|c|}
\hline C(21)-C(13)-C(12) & 113.4(3) \\
\hline$C(15)-C(14)-C(8)$ & 106.8(3) \\
\hline C(15)-C(14)-C(13) & 113.8(3) \\
\hline C(8)-C(14)-C(13) & 108.4(3) \\
\hline $\mathrm{C}(16)-\mathrm{C}(15)-\mathrm{C}(14)$ & $110.7(3)$ \\
\hline $\mathrm{O}(27)-\mathrm{C}(16)-\mathrm{O}(17)$ & 108.1(3) \\
\hline $\mathrm{O}(27)-\mathrm{C}(16)-\mathrm{C}(15)$ & 107.7(3) \\
\hline $\mathrm{O}(17)-\mathrm{C}(16)-\mathrm{C}(15)$ & 112.6(3) \\
\hline $\mathrm{O}(23)-\mathrm{C}(22)-\mathrm{O}(24)$ & 123.4(4) \\
\hline $\mathrm{O}(23)-\mathrm{C}(22)-\mathrm{C}(12)$ & $124.7(3)$ \\
\hline $\mathrm{O}(24)-\mathrm{C}(22)-\mathrm{C}(12)$ & 111.8(4) \\
\hline O(27)-C(28)-C(29) & 107.1(4) \\
\hline $\mathrm{O}(31)-\mathrm{C}(30)-\mathrm{O}(32)$ & 124.4(4) \\
\hline $\mathrm{O}(31)-\mathrm{C}(30)-\mathrm{C}(39)$ & $125.0(4)$ \\
\hline O(32)-C(30)-C(39) & $110.6(4)$ \\
\hline $\mathrm{C}(34)-\mathrm{C}(33)-\mathrm{O}(32)$ & 109.5(5) \\
\hline $\mathrm{C}(38)-\mathrm{C}(37)-\mathrm{O}(36)$ & 107.6(4) \\
\hline C(3)-C(39)-C(11) & 112.0(3) \\
\hline C(3)-C(39)-C(30) & 108.7(3) \\
\hline C(11)-C(39)-C(30) & 109.9(3) \\
\hline C(3)-C(39)-S(2) & 106.0(3) \\
\hline C(11)-C(39)-S(2) & 110.4(3) \\
\hline C(30)-C(39)-S(2) & 109.7(3) \\
\hline $\mathrm{C}(7)-\mathrm{O}(17)-\mathrm{C}(16)$ & 118.0(3) \\
\hline C(30)-O(32)-C(33) & 115.4(4) \\
\hline $\mathrm{C}(3)-\mathrm{O}(36)-\mathrm{C}(37)$ & 115.8(3) \\
\hline $\mathrm{C}(16)-\mathrm{O}(27)-\mathrm{C}(28)$ & 114.8(3) \\
\hline C(1)-S(2)-C(39) & $96.28(19$ \\
\hline
\end{tabular}

Symmetry transformations used to generate equivalent atoms:

Table 4. Anisotropic displacement parameters $\left(\AA^{2} \times 10^{3}\right)$ for sp3-053tpièce. The anisotropic displacement factor exponent takes the form: $-2 \pi^{2}\left[h^{2} a^{* 2} U^{11}+\ldots+2 h k a^{*} b^{*} U^{12}\right]$

\begin{tabular}{lcccccc}
\hline & $\mathrm{U}^{11}$ & $\mathrm{U}^{22}$ & $\mathrm{U}^{33}$ & $\mathrm{U}^{23}$ & $\mathrm{U}^{13}$ & $\mathrm{U}^{12}$ \\
\hline $\mathrm{O}(24)$ & $129(2)$ & $120(2)$ & $84(2)$ & $8(2)$ & $-35(2)$ & $29(2)$ \\
$\mathrm{C}(25)$ & $191(6)$ & $165(5)$ & $131(4)$ & $11(4)$ & $-59(4)$ & $77(5)$ \\
$\mathrm{C}(26)$ & $201(7)$ & $257(9)$ & $241(9)$ & $27(7)$ & $-136(7)$ & $36(7)$
\end{tabular}




\begin{tabular}{|c|c|c|c|c|c|c|}
\hline C(38) & $367(12)$ & $149(6)$ & 135(5) & $31(4)$ & $9(6)$ & $128(7)$ \\
\hline $\mathrm{C}(1)$ & 91(3) & $113(3)$ & $112(3)$ & $8(3)$ & $-7(2)$ & $14(2)$ \\
\hline C(3) & $98(3)$ & $86(3)$ & 82(3) & $5(2)$ & $-12(2)$ & $-13(2)$ \\
\hline $\mathrm{C}(7)$ & 88(3) & 94(3) & $94(3)$ & $8(2)$ & $-18(2)$ & $-10(2)$ \\
\hline C(8) & $80(2)$ & $74(2)$ & $80(2)$ & $7(2)$ & $-17(2)$ & $-1(2)$ \\
\hline C(9) & $80(2)$ & $75(2)$ & $83(2)$ & $5(2)$ & $-10(2)$ & $-2(2)$ \\
\hline C(10) & 81(3) & $90(3)$ & 106(3) & $10(2)$ & $-15(2)$ & $8(2)$ \\
\hline C(11) & $82(2)$ & $74(2)$ & $69(2)$ & $-2(2)$ & $-10(2)$ & $4(2)$ \\
\hline $\mathrm{C}(12)$ & $75(2)$ & $72(2)$ & $69(2)$ & $-2(2)$ & $-13(2)$ & $5(2)$ \\
\hline C(13) & $82(2)$ & $77(2)$ & $76(2)$ & $-10(2)$ & $-11(2)$ & $4(2)$ \\
\hline C(14) & $75(2)$ & $75(2)$ & $78(2)$ & $-2(2)$ & $-13(2)$ & $-1(2)$ \\
\hline C(15) & $87(3)$ & 89(3) & 87(3) & $-2(2)$ & $-7(2)$ & $-8(2)$ \\
\hline C(16) & $95(3)$ & 81(3) & 90(3) & $6(2)$ & $-7(2)$ & $-3(2)$ \\
\hline C(18) & $115(4)$ & $125(4)$ & $152(4)$ & $47(3)$ & $-4(3)$ & $35(3)$ \\
\hline C(21) & $92(3)$ & $105(3)$ & $81(2)$ & $-7(2)$ & $-13(2)$ & $-12(2)$ \\
\hline C(22) & $90(3)$ & $86(3)$ & 79(3) & $-1(2)$ & $-12(2)$ & 1(2) \\
\hline C(28) & $110(3)$ & $126(4)$ & $85(3)$ & $-3(3)$ & $6(2)$ & $-7(3)$ \\
\hline C(29) & 161(5) & $121(4)$ & $143(4)$ & 28(3) & $31(4)$ & $-3(4)$ \\
\hline C(30) & $83(3)$ & $97(3)$ & 99(3) & $5(2)$ & $-20(2)$ & $-8(2)$ \\
\hline C(33) & 139(4) & $160(5)$ & $135(4)$ & $6(4)$ & $-46(3)$ & $-75(4)$ \\
\hline C(34) & 245(8) & $122(5)$ & 203(7) & $6(5)$ & $-51(6)$ & $-61(5)$ \\
\hline C(37) & $174(5)$ & $99(3)$ & $110(4)$ & 31(3) & $-11(3)$ & $16(3)$ \\
\hline C(39) & $82(2)$ & $79(2)$ & $81(2)$ & $0(2)$ & $-7(2)$ & $-2(2)$ \\
\hline $\mathrm{O}(17)$ & $106(2)$ & $124(2)$ & $84(2)$ & $10(2)$ & $-14(2)$ & $-18(2)$ \\
\hline $\mathrm{O}(23)$ & $106(2)$ & $107(2)$ & $103(2)$ & $-4(2)$ & $-17(2)$ & $27(2)$ \\
\hline $\mathrm{O}(31)$ & $118(2)$ & $130(2)$ & $82(2)$ & $0(2)$ & $-19(2)$ & $-28(2)$ \\
\hline O(32) & $109(2)$ & $124(2)$ & $107(2)$ & $6(2)$ & $-15(2)$ & $-41(2)$ \\
\hline O(35) & $160(3)$ & $117(2)$ & $76(2)$ & $5(2)$ & $0(2)$ & $0(2)$ \\
\hline $\mathrm{O}(36)$ & $129(2)$ & $85(2)$ & $88(2)$ & $12(2)$ & $-12(2)$ & $10(2)$ \\
\hline $\mathrm{O}(27)$ & $116(2)$ & $89(2)$ & $96(2)$ & $-2(2)$ & $11(2)$ & $-5(2)$ \\
\hline$S(2)$ & 89(1) & 108(1) & 107(1) & $-3(1)$ & $5(1)$ & $7(1)$ \\
\hline
\end{tabular}


Table 5. Hydrogen coordinates ( x $\left.10^{4}\right)$ and isotropic displacement parameters $\left(\AA^{2} \mathrm{x} 10^{3}\right)$ for sp3-053tpièce.

\begin{tabular}{|c|c|c|c|c|}
\hline & $\mathrm{x}$ & $\mathrm{y}$ & $\mathrm{z}$ & $\mathrm{U}(\mathrm{eq})$ \\
\hline $\mathrm{H}(25 \mathrm{~A})$ & 10521 & 1795 & 2331 & 199 \\
\hline $\mathrm{H}(25 \mathrm{~B})$ & 11488 & 2712 & 2042 & 199 \\
\hline $\mathrm{H}(26 \mathrm{~A})$ & 12431 & 2682 & 2752 & 361 \\
\hline $\mathrm{H}(26 \mathrm{~B})$ & 10927 & 3063 & 2937 & 361 \\
\hline $\mathrm{H}(26 \mathrm{C})$ & 11766 & 4093 & 2652 & 361 \\
\hline $\mathrm{H}(38 \mathrm{~A})$ & 8337 & -1035 & 1974 & 326 \\
\hline $\mathrm{H}(38 \mathrm{~B})$ & 8335 & -162 & 1529 & 326 \\
\hline $\mathrm{H}(38 \mathrm{C})$ & 6962 & -1017 & 1621 & 326 \\
\hline $\mathrm{H}(1 \mathrm{~A})$ & 3213 & 5084 & 961 & 127 \\
\hline $\mathrm{H}(1 \mathrm{~B})$ & 3346 & 6435 & 1224 & 127 \\
\hline $\mathrm{H}(11)$ & 7307 & 3576 & 1046 & 91 \\
\hline $\mathrm{H}(12)$ & 7538 & 4894 & 1888 & 88 \\
\hline $\mathrm{H}(13)$ & 8140 & 6702 & 1481 & 95 \\
\hline $\mathrm{H}(14)$ & 9696 & 5041 & 906 & 92 \\
\hline $\mathrm{H}(15 \mathrm{~A})$ & 9441 & 7834 & 828 & 106 \\
\hline $\mathrm{H}(15 \mathrm{~B})$ & 10919 & 7089 & 952 & 106 \\
\hline $\mathrm{H}(16)$ & 10884 & 6151 & 233 & 108 \\
\hline $\mathrm{H}(18 \mathrm{~A})$ & 5628 & 7709 & 529 & 197 \\
\hline $\mathrm{H}(18 \mathrm{~B})$ & 4019 & 7713 & 675 & 197 \\
\hline $\mathrm{H}(18 \mathrm{C})$ & 4433 & 6852 & 256 & 197 \\
\hline $\mathrm{H}(21 \mathrm{~A})$ & 9941 & 6281 & 2090 & 140 \\
\hline $\mathrm{H}(21 \mathrm{~B})$ & 10529 & 7164 & 1701 & 140 \\
\hline $\mathrm{H}(21 \mathrm{C})$ & 10934 & 5656 & 1732 & 140 \\
\hline $\mathrm{H}(28 \mathrm{~A})$ & 10572 & 7840 & -557 & 128 \\
\hline $\mathrm{H}(28 \mathrm{~B})$ & 12046 & 7263 & -328 & 128 \\
\hline $\mathrm{H}(29 \mathrm{~A})$ & 12425 & 9283 & -698 & 211 \\
\hline $\mathrm{H}(29 \mathrm{~B})$ & 12898 & 9370 & -171 & 211 \\
\hline $\mathrm{H}(29 \mathrm{C})$ & 11405 & 9978 & -363 & 211 \\
\hline $\mathrm{H}(33 \mathrm{~A})$ & 2047 & 1748 & 728 & 177 \\
\hline $\mathrm{H}(33 \mathrm{~B})$ & 3395 & 811 & 682 & 177 \\
\hline $\mathrm{H}(34 \mathrm{~A})$ & 2807 & -322 & 1296 & 290 \\
\hline $\mathrm{H}(34 \mathrm{~B})$ & 1508 & -315 & 915 & 290 \\
\hline $\mathrm{H}(34 \mathrm{C})$ & 1466 & 615 & 1345 & 290 \\
\hline $\mathrm{H}(37 \mathrm{~A})$ & 7914 & 944 & 2217 & 154 \\
\hline
\end{tabular}




\begin{tabular}{lllll}
$\mathrm{H}(37 \mathrm{~B})$ & 6361 & 277 & 2169 & 154 \\
$\mathrm{H}(7)$ & $6720(50)$ & $6080(40)$ & $21(14)$ & $120(14)$ \\
\hline
\end{tabular}

Table 6. Torsion angles $\left[{ }^{\circ}\right]$ for sp3-053tpièce.

\begin{tabular}{|c|c|}
\hline $\mathrm{C}(22)-\mathrm{O}(24)-\mathrm{C}(25)-\mathrm{C}(26)$ & $150.6(6)$ \\
\hline O(17)-C(7)-C(8)-C(9) & 175.0(4) \\
\hline O(17)-C(7)-C(8)-C(14) & $-1.1(6)$ \\
\hline C(7)-C(8)-C(9)-C(10) & $-43.4(6)$ \\
\hline C(14)-C(8)-C(9)-C(10) & $132.9(4)$ \\
\hline C(7)-C(8)-C(9)-C(11) & 141.1(4) \\
\hline C(14)-C(8)-C(9)-C(11) & $-42.6(4)$ \\
\hline C(8)-C(9)-C(10)-C(1) & $-176.3(4)$ \\
\hline C(11)-C(9)-C(10)-C(1) & $-1.6(6)$ \\
\hline C(8)-C(9)-C(10)-C(18) & $4.2(7)$ \\
\hline C(11)-C(9)-C(10)-C(18) & $178.9(4)$ \\
\hline S(2)-C(1)-C(10)-C(9) & 23.9(6) \\
\hline S(2)-C(1)-C(10)-C(18) & $-156.6(4)$ \\
\hline C(10)-C(9)-C(11)-C(39) & $16.5(5)$ \\
\hline C(8)-C(9)-C(11)-C(39) & $-168.1(3)$ \\
\hline C(10)-C(9)-C(11)-C(12) & $-111.5(4)$ \\
\hline C(8)-C(9)-C(11)-C(12) & $63.9(4)$ \\
\hline C(9)-C(11)-C(12)-C(22) & $-140.9(3)$ \\
\hline $\mathrm{C}(39)-\mathrm{C}(11)-\mathrm{C}(12)-\mathrm{C}(22)$ & $90.3(4)$ \\
\hline C(9)-C(11)-C(12)-C(13) & $-20.1(4)$ \\
\hline C(39)-C(11)-C(12)-C(13) & $-148.9(3)$ \\
\hline $\mathrm{C}(22)-\mathrm{C}(12)-\mathrm{C}(13)-\mathrm{C}(14)$ & $80.8(3)$ \\
\hline $\mathrm{C}(11)-\mathrm{C}(12)-\mathrm{C}(13)-\mathrm{C}(14)$ & $-41.7(4)$ \\
\hline $\mathrm{C}(22)-\mathrm{C}(12)-\mathrm{C}(13)-\mathrm{C}(21)$ & $-46.5(4)$ \\
\hline $\mathrm{C}(11)-\mathrm{C}(12)-\mathrm{C}(13)-\mathrm{C}(21)$ & $-168.9(3)$ \\
\hline C(7)-C(8)-C(14)-C(15) & $33.6(5)$ \\
\hline C(9)-C(8)-C(14)-C(15) & $-142.9(3)$ \\
\hline C(7)-C(8)-C(14)-C(13) & $156.7(4)$ \\
\hline C(9)-C(8)-C(14)-C(13) & $-19.8(4)$ \\
\hline C(21)-C(13)-C(14)-C(15) & $-50.3(4)$ \\
\hline $\mathrm{C}(12)-\mathrm{C}(13)-\mathrm{C}(14)-\mathrm{C}(15)$ & $-177.9(3)$ \\
\hline C(21)-C(13)-C(14)-C(8) & $-169.0(3)$ \\
\hline C(12)-C(13)-C(14)-C(8) & $63.4(4)$ \\
\hline C(8)-C(14)-C(15)-C(16) & $-58.0(4)$ \\
\hline
\end{tabular}




\begin{tabular}{|c|c|}
\hline C(13)-C(14)-C(15)-C(16) & $-177.6(3)$ \\
\hline $\mathrm{C}(14)-\mathrm{C}(15)-\mathrm{C}(16)-\mathrm{O}(27)$ & $171.7(3)$ \\
\hline $\mathrm{C}(14)-\mathrm{C}(15)-\mathrm{C}(16)-\mathrm{O}(17)$ & $52.6(4)$ \\
\hline $\mathrm{C}(25)-\mathrm{O}(24)-\mathrm{C}(22)-\mathrm{O}(23)$ & $9.3(6)$ \\
\hline $\mathrm{C}(25)-\mathrm{O}(24)-\mathrm{C}(22)-\mathrm{C}(12)$ & $-168.8(4)$ \\
\hline $\mathrm{C}(11)-\mathrm{C}(12)-\mathrm{C}(22)-\mathrm{O}(23)$ & $41.7(5)$ \\
\hline $\mathrm{C}(13)-\mathrm{C}(12)-\mathrm{C}(22)-\mathrm{O}(23)$ & $-81.0(5)$ \\
\hline $\mathrm{C}(11)-\mathrm{C}(12)-\mathrm{C}(22)-\mathrm{O}(24)$ & $-140.2(3)$ \\
\hline $\mathrm{C}(13)-\mathrm{C}(12)-\mathrm{C}(22)-\mathrm{O}(24)$ & $97.1(4)$ \\
\hline O(35)-C(3)-C(39)-C(11) & 109.9(4) \\
\hline O(36)-C(3)-C(39)-C(11) & $-69.9(4)$ \\
\hline $\mathrm{O}(35)-\mathrm{C}(3)-\mathrm{C}(39)-\mathrm{C}(30)$ & $-128.4(4)$ \\
\hline O(36)-C(3)-C(39)-C(30) & $51.7(4)$ \\
\hline $\mathrm{O}(35)-\mathrm{C}(3)-\mathrm{C}(39)-\mathrm{S}(2)$ & $-10.5(5)$ \\
\hline $\mathrm{O}(36)-\mathrm{C}(3)-\mathrm{C}(39)-\mathrm{S}(2)$ & $169.6(3)$ \\
\hline C(9)-C(11)-C(39)-C(3) & $-168.4(3)$ \\
\hline C(12)-C(11)-C(39)-C(3) & $-41.9(4)$ \\
\hline C(9)-C(11)-C(39)-C(30) & $70.6(4)$ \\
\hline C(12)-C(11)-C(39)-C(30) & $-162.9(3)$ \\
\hline C(9)-C(11)-C(39)-S(2) & $-50.5(3)$ \\
\hline C(12)-C(11)-C(39)-S(2) & 75.9(3) \\
\hline $\mathrm{O}(31)-\mathrm{C}(30)-\mathrm{C}(39)-\mathrm{C}(3)$ & $-129.6(5)$ \\
\hline $\mathrm{O}(32)-\mathrm{C}(30)-\mathrm{C}(39)-\mathrm{C}(3)$ & $48.5(4)$ \\
\hline $\mathrm{O}(31)-\mathrm{C}(30)-\mathrm{C}(39)-\mathrm{C}(11)$ & $-6.7(6)$ \\
\hline $\mathrm{O}(32)-\mathrm{C}(30)-\mathrm{C}(39)-\mathrm{C}(11)$ & $171.4(3)$ \\
\hline $\mathrm{O}(31)-\mathrm{C}(30)-\mathrm{C}(39)-\mathrm{S}(2)$ & $114.9(4)$ \\
\hline O(32)-C(30)-C(39)-S(2) & $-67.0(4)$ \\
\hline C(8)-C(7)-O(17)-C(16) & $-7.3(6)$ \\
\hline $\mathrm{O}(27)-\mathrm{C}(16)-\mathrm{O}(17)-\mathrm{C}(7)$ & $-138.0(3)$ \\
\hline $\mathrm{C}(15)-\mathrm{C}(16)-\mathrm{O}(17)-\mathrm{C}(7)$ & $-19.1(5)$ \\
\hline $\mathrm{O}(31)-\mathrm{C}(30)-\mathrm{O}(32)-\mathrm{C}(33)$ & $-0.2(6)$ \\
\hline C(39)-C(30)-O(32)-C(33) & $-178.3(4)$ \\
\hline $\mathrm{C}(34)-\mathrm{C}(33)-\mathrm{O}(32)-\mathrm{C}(30)$ & $162.5(5)$ \\
\hline $\mathrm{O}(35)-\mathrm{C}(3)-\mathrm{O}(36)-\mathrm{C}(37)$ & $1.0(6)$ \\
\hline C(39)-C(3)-O(36)-C(37) & $-179.1(4)$ \\
\hline $\mathrm{C}(38)-\mathrm{C}(37)-\mathrm{O}(36)-\mathrm{C}(3)$ & $175.9(6)$ \\
\hline $\mathrm{O}(17)-\mathrm{C}(16)-\mathrm{O}(27)-\mathrm{C}(28)$ & $-71.3(4)$ \\
\hline $\mathrm{C}(15)-\mathrm{C}(16)-\mathrm{O}(27)-\mathrm{C}(28)$ & $166.8(3)$ \\
\hline $\mathrm{C}(29)-\mathrm{C}(28)-\mathrm{O}(27)-\mathrm{C}(16)$ & $-172.9(4)$ \\
\hline C(10)-C(1)-S(2)-C(39) & $-49.4(4)$ \\
\hline
\end{tabular}


C(3)-C(39)-S(2)-C(1)

$\mathrm{C}(11)-\mathrm{C}(39)-\mathrm{S}(2)-\mathrm{C}(1)$

$\mathrm{C}(30)-\mathrm{C}(39)-\mathrm{S}(2)-\mathrm{C}(1)$
$-176.0(3)$

$62.5(3)$

$-58.7(3)$

Symmetry transformations used to generate equivalent atoms: 
Dioxane-4,6-dione 19c (CCDC 600919)

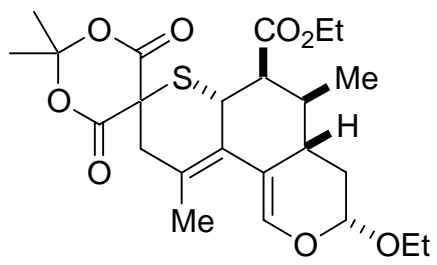

Experimental : The crystals were grown by slow evaporation of an ethanol solution at room temperature. One single crystal was mounted using a glass fiber on the goniometer. Data were collected on an Enraf-Nonius CAD-4 automatic diffractometer at the Université de Sherbrooke using $\omega$ scans at 293(2) K. The DIFRAC ${ }^{(1)}$ program was used for centering, indexing, and data collection. Two standard reflections were measured every 100 reflections, no intensity decay was observed during data collection. The data were corrected for absorption by empirical methods based on psi scans and reduced with the NRCVAX ${ }^{(2)}$ programs. They were solved using SHELXS-97(3) and refined by full-matrix least squares on $\mathrm{F}^{2}$ with SHELXL-97(4). The non-hydrogen atoms were refined anisotropically. The hydrogen atoms were placed at idealized calculated geometric position and refined isotropically using a riding model, but H11, 13, 14, 16, 20 and 21 were found by Fourier differences.

(1) H.D. Flack, E. Blanc and D. Schwarzenbach (1992), J. Appl. Cryst., 25, 455-459.

(2) E.J. Gabe, Y. Le Page, J.-P. Charland, F.L. Lee, and P.S. White, (1989) J. Appl. Cryst., 22, 384-387.

(3) G. M. Sheldrick, SHELXS-97, G.M. Sheldrick, University of Göttingen, Germany, 1997, Release 97-2.

(4) G. M. Sheldrick, SHELXL-97, G.M. Sheldrick, University of Göttingen, Germany, 1997, Release 97-2. 


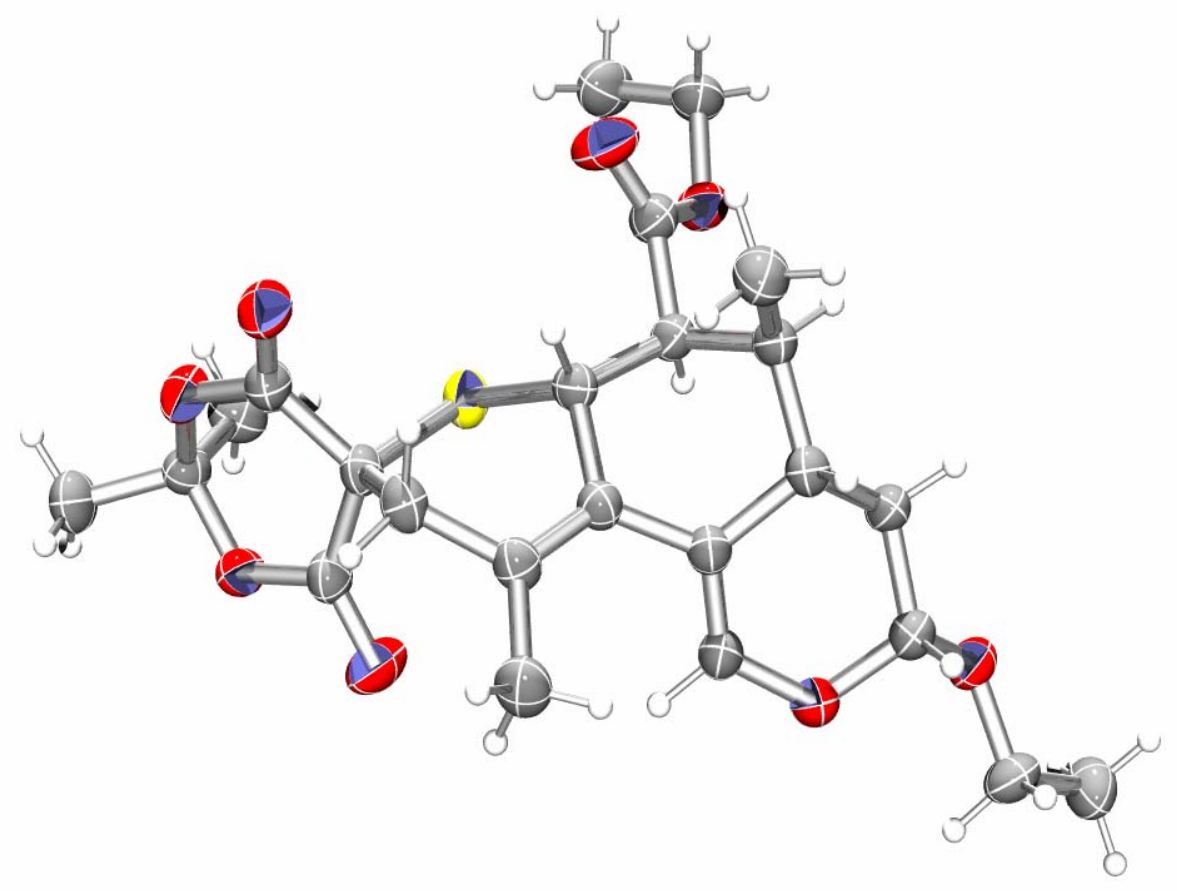

Ellipsoid probability set at $30 \%$.

Table 1. Crystal data and structure refinement for sp4.

Identification code

sp4

Empirical formula

C24 H32 O8 S

Formula weight

480.56

Temperature

293(2) K

Wavelength

$1.54176 \AA$

Crystal system

Monoclinic

Space group

P 1 21/n 1

Unit cell dimensions

$\mathrm{a}=6.0436(8) \AA$
$\mathrm{b}=23.163(4) \AA$
$\mathrm{c}=17.364(3) \AA$

$\alpha=90^{\circ}$.

2430.4(7) $\AA^{3}$

Volume

4

Z

$1.313 \mathrm{Mg} / \mathrm{m}^{3}$

Density (calculated)

$1.577 \mathrm{~mm}^{-1}$

$\mathrm{F}(000)$

1024 
Crystal size

Theta range for data collection

Index ranges

Reflections collected

Independent reflections

Completeness to theta $=70.16^{\circ}$

Absorption correction

Max. and min. transmission

Refinement method

Data / restraints / parameters

Goodness-of-fit on $\mathrm{F}^{2}$

Final R indices [I $>2 \operatorname{sigma(I)]~}$

$\mathrm{R}$ indices (all data)

Extinction coefficient

Largest diff. peak and hole
$0.4 \times 0.1 \times 0.1 \mathrm{~mm}^{3}$

3.18 to $70.16^{\circ}$.

$-7<=\mathrm{h}<=7,0<=\mathrm{k}<=28,0<=\mathrm{l}<=21$

4518

$4518[\mathrm{R}(\mathrm{int})=0.0000]$

$97.7 \%$

Empirical

0.9945 and 0.9001

Full-matrix least-squares on $\mathrm{F}^{2}$

4518 / 0 / 329

0.978

$\mathrm{R} 1=0.0588, \mathrm{wR} 2=0.1406$

$\mathrm{R} 1=0.1386, \mathrm{wR} 2=0.1703$

0.0067(6)

0.184 and -0.250 e. $\AA^{-3}$

Table 2. Atomic coordinates ( $\left.\mathrm{x} 10^{4}\right)$ and equivalent isotropic displacement parameters $\left(\AA^{2} \mathrm{x} 10^{3}\right)$ for sp4. $\mathrm{U}(\mathrm{eq})$ is defined as one third of the trace of the orthogonalized $\mathrm{U}^{\mathrm{ij}}$ tensor.

\begin{tabular}{|c|c|c|c|c|}
\hline & $\mathrm{x}$ & $\mathrm{y}$ & $\mathrm{z}$ & $\mathrm{U}(\mathrm{eq})$ \\
\hline $\mathrm{C}(1)$ & $7148(8)$ & $9339(2)$ & $4440(2)$ & $89(1)$ \\
\hline $\mathrm{C}(2)$ & 7866(7) & $9830(2)$ & $3936(2)$ & $64(1)$ \\
\hline $\mathrm{C}(3)$ & 10081(7) & $9740(2)$ & $3582(2)$ & $85(1)$ \\
\hline $\mathrm{C}(4)$ & $7570(7)$ & $10867(2)$ & $4129(2)$ & $66(1)$ \\
\hline $\mathrm{C}(5)$ & $6714(6)$ & 10928(1) & $3311(2)$ & $56(1)$ \\
\hline C(6) & $5678(7)$ & $10377(2)$ & 2996(2) & $65(1)$ \\
\hline $\mathrm{C}(7)$ & 4957(6) & $11410(1)$ & $3264(2)$ & $67(1)$ \\
\hline$C(8)$ & $5837(6)$ & 12021(2) & $3357(2)$ & $62(1)$ \\
\hline C(9) & $4940(7)$ & $12369(2)$ & $4001(2)$ & $82(1)$ \\
\hline$C(10)$ & 7383(6) & 12188(1) & 2859(2) & $57(1)$ \\
\hline $\mathrm{C}(11)$ & $8074(6)$ & $11763(2)$ & $2251(2)$ & $58(1)$ \\
\hline$C(12)$ & $8652(6)$ & $12725(1)$ & $2888(2)$ & $61(1)$ \\
\hline$C(13)$ & $9244(7)$ & 13031(2) & 2152(2) & $64(1)$ \\
\hline $\mathrm{C}(14)$ & $9253(7)$ & $12612(2)$ & $1464(2)$ & $67(1)$ \\
\hline$C(15)$ & 7064(8) & $12614(2)$ & $1007(2)$ & $90(1)$ \\
\hline$C(16)$ & $9908(7)$ & $12007(2)$ & $1758(2)$ & $60(1)$ \\
\hline $\mathrm{C}(17)$ & 10494(7) & $11616(2)$ & 1098(2) & $71(1)$ \\
\hline $\mathrm{C}(18)$ & $13518(7)$ & $11318(2)$ & $315(2)$ & $79(1)$ \\
\hline
\end{tabular}




\begin{tabular}{lrrrr}
$\mathrm{C}(19)$ & $14056(9)$ & $10723(2)$ & $538(3)$ & $99(2)$ \\
$\mathrm{C}(20)$ & $9539(7)$ & $12909(2)$ & $3550(2)$ & $68(1)$ \\
$\mathrm{C}(21)$ & $11454(7)$ & $13708(2)$ & $2978(2)$ & $68(1)$ \\
$\mathrm{C}(22)$ & $13702(8)$ & $14348(2)$ & $3715(2)$ & $84(1)$ \\
$\mathrm{C}(23)$ & $15883(8)$ & $14638(2)$ & $3681(3)$ & $100(2)$ \\
$\mathrm{C}(24)$ & $11460(7)$ & $13336(2)$ & $2270(2)$ & $69(1)$ \\
$\mathrm{O}(1)$ & $7883(5)$ & $10332(1)$ & $4411(1)$ & $71(1)$ \\
$\mathrm{O}(2)$ & $6174(4)$ & $9877(1)$ & $3343(1)$ & $75(1)$ \\
$\mathrm{O}(3)$ & $7879(6)$ & $11262(1)$ & $4563(2)$ & $101(1)$ \\
$\mathrm{O}(4)$ & $4389(5)$ & $10371(1)$ & $2470(2)$ & $84(1)$ \\
$\mathrm{O}(5)$ & $12680(5)$ & $11646(1)$ & $968(1)$ & $75(1)$ \\
$\mathrm{O}(6)$ & $9235(5)$ & $11335(1)$ & $724(2)$ & $102(1)$ \\
$\mathrm{O}(7)$ & $13558(5)$ & $13924(1)$ & $3107(1)$ & $73(1)$ \\
$\mathrm{O}(8)$ & $10876(5)$ & $13381(1)$ & $3653(1)$ & $80(1)$ \\
$\mathrm{S}(1)$ & $9089(2)$ & $11098(1)$ & $2708(1)$ & $69(1)$ \\
\hline
\end{tabular}

Table 3. Bond lengths $[\AA]$ and angles $\left[{ }^{\circ}\right]$ for sp4.

\begin{tabular}{ll}
\hline $\mathrm{C}(1)-\mathrm{C}(2)$ & $1.502(5)$ \\
$\mathrm{C}(2)-\mathrm{O}(1)$ & $1.426(4)$ \\
$\mathrm{C}(2)-\mathrm{O}(2)$ & $1.443(4)$ \\
$\mathrm{C}(2)-\mathrm{C}(3)$ & $1.497(5)$ \\
$\mathrm{C}(4)-\mathrm{O}(3)$ & $1.199(4)$ \\
$\mathrm{C}(4)-\mathrm{O}(1)$ & $1.344(4)$ \\
$\mathrm{C}(4)-\mathrm{C}(5)$ & $1.509(5)$ \\
$\mathrm{C}(5)-\mathrm{C}(6)$ & $1.519(5)$ \\
$\mathrm{C}(5)-\mathrm{C}(7)$ & $1.543(5)$ \\
$\mathrm{C}(5)-\mathrm{S}(1)$ & $1.834(4)$ \\
$\mathrm{C}(6)-\mathrm{O}(4)$ & $1.191(4)$ \\
$\mathrm{C}(6)-\mathrm{O}(2)$ & $1.337(4)$ \\
$\mathrm{C}(7)-\mathrm{C}(8)$ & $1.518(5)$ \\
$\mathrm{C}(8)-\mathrm{C}(10)$ & $1.339(4)$ \\
$\mathrm{C}(8)-\mathrm{C}(9)$ & $1.490(5)$ \\
$\mathrm{C}(10)-\mathrm{C}(12)$ & $1.462(5)$ \\
$\mathrm{C}(10)-\mathrm{C}(11)$ & $1.508(5)$ \\
$\mathrm{C}(11)-\mathrm{C}(16)$ & $1.520(5)$ \\
$\mathrm{C}(11)-\mathrm{S}(1)$ & $1.834(4)$ \\
$\mathrm{C}(12)-\mathrm{C}(20)$ & $1.331(5)$ \\
$\mathrm{C}(12)-\mathrm{C}(13)$ & $1.511(5)$ \\
\end{tabular}




\begin{tabular}{|c|c|}
\hline C(13)-C(24) & $1.525(5)$ \\
\hline C(13)-C(14) & $1.539(5)$ \\
\hline $\mathrm{C}(14)-\mathrm{C}(15)$ & $1.532(6)$ \\
\hline$C(14)-C(16)$ & $1.542(5)$ \\
\hline$C(16)-C(17)$ & $1.507(5)$ \\
\hline $\mathrm{C}(17)-\mathrm{O}(6)$ & $1.187(4)$ \\
\hline $\mathrm{C}(17)-\mathrm{O}(5)$ & $1.346(5)$ \\
\hline $\mathrm{C}(18)-\mathrm{O}(5)$ & $1.463(4)$ \\
\hline $\mathrm{C}(18)-\mathrm{C}(19)$ & $1.467(5)$ \\
\hline $\mathrm{C}(20)-\mathrm{O}(8)$ & $1.368(4)$ \\
\hline $\mathrm{C}(21)-\mathrm{O}(7)$ & $1.382(5)$ \\
\hline $\mathrm{C}(21)-\mathrm{O}(8)$ & $1.443(4)$ \\
\hline$C(21)-C(24)$ & $1.502(5)$ \\
\hline $\mathrm{C}(22)-\mathrm{O}(7)$ & $1.443(4)$ \\
\hline$C(22)-C(23)$ & $1.482(6)$ \\
\hline $\mathrm{O}(1)-\mathrm{C}(2)-\mathrm{O}(2)$ & $110.4(3)$ \\
\hline $\mathrm{O}(1)-\mathrm{C}(2)-\mathrm{C}(3)$ & $110.6(3)$ \\
\hline $\mathrm{O}(2)-\mathrm{C}(2)-\mathrm{C}(3)$ & $110.2(3)$ \\
\hline $\mathrm{O}(1)-\mathrm{C}(2)-\mathrm{C}(1)$ & $106.3(3)$ \\
\hline $\mathrm{O}(2)-\mathrm{C}(2)-\mathrm{C}(1)$ & $105.4(3)$ \\
\hline$C(3)-C(2)-C(1)$ & $113.8(3)$ \\
\hline $\mathrm{O}(3)-\mathrm{C}(4)-\mathrm{O}(1)$ & $117.0(3)$ \\
\hline $\mathrm{O}(3)-\mathrm{C}(4)-\mathrm{C}(5)$ & $124.6(3)$ \\
\hline $\mathrm{O}(1)-\mathrm{C}(4)-\mathrm{C}(5)$ & $118.2(3)$ \\
\hline$C(4)-C(5)-C(6)$ & 113.1(3) \\
\hline$C(4)-C(5)-C(7)$ & $110.0(3)$ \\
\hline$C(6)-C(5)-C(7)$ & $108.1(3)$ \\
\hline$C(4)-C(5)-S(1)$ & $107.4(3)$ \\
\hline$C(6)-C(5)-S(1)$ & $107.3(2)$ \\
\hline$C(7)-C(5)-S(1)$ & $111.0(2)$ \\
\hline $\mathrm{O}(4)-\mathrm{C}(6)-\mathrm{O}(2)$ & $118.4(3)$ \\
\hline $\mathrm{O}(4)-\mathrm{C}(6)-\mathrm{C}(5)$ & 123.1(3) \\
\hline $\mathrm{O}(2)-\mathrm{C}(6)-\mathrm{C}(5)$ & 118.5(3) \\
\hline$C(8)-C(7)-C(5)$ & $115.5(3)$ \\
\hline $\mathrm{C}(10)-\mathrm{C}(8)-\mathrm{C}(9)$ & $126.5(3)$ \\
\hline $\mathrm{C}(10)-\mathrm{C}(8)-\mathrm{C}(7)$ & 116.6(3) \\
\hline $\mathrm{C}(9)-\mathrm{C}(8)-\mathrm{C}(7)$ & $116.9(3)$ \\
\hline$C(8)-C(10)-C(12)$ & $126.5(3)$ \\
\hline $\mathrm{C}(8)-\mathrm{C}(10)-\mathrm{C}(11)$ & $117.9(3)$ \\
\hline
\end{tabular}




$\begin{array}{ll}\mathrm{C}(12)-\mathrm{C}(10)-\mathrm{C}(11) & 115.3(3) \\ \mathrm{C}(10)-\mathrm{C}(11)-\mathrm{C}(16) & 111.4(3) \\ \mathrm{C}(10)-\mathrm{C}(11)-\mathrm{S}(1) & 109.8(2) \\ \mathrm{C}(16)-\mathrm{C}(11)-\mathrm{S}(1) & 108.3(3) \\ \mathrm{C}(20)-\mathrm{C}(12)-\mathrm{C}(10) & 120.4(3) \\ \mathrm{C}(20)-\mathrm{C}(12)-\mathrm{C}(13) & 118.8(3) \\ \mathrm{C}(10)-\mathrm{C}(12)-\mathrm{C}(13) & 120.2(3) \\ \mathrm{C}(12)-\mathrm{C}(13)-\mathrm{C}(24) & 108.9(3) \\ \mathrm{C}(12)-\mathrm{C}(13)-\mathrm{C}(14) & 111.3(3) \\ \mathrm{C}(24)-\mathrm{C}(13)-\mathrm{C}(14) & 112.4(3) \\ \mathrm{C}(15)-\mathrm{C}(14)-\mathrm{C}(13) & 112.7(4) \\ \mathrm{C}(15)-\mathrm{C}(14)-\mathrm{C}(16) & 112.9(3) \\ \mathrm{C}(13)-\mathrm{C}(14)-\mathrm{C}(16) & 108.8(3) \\ \mathrm{C}(17)-\mathrm{C}(16)-\mathrm{C}(11) & 112.8(3) \\ \mathrm{C}(17)-\mathrm{C}(16)-\mathrm{C}(14) & 110.9(3) \\ \mathrm{C}(11)-\mathrm{C}(16)-\mathrm{C}(14) & 109.7(3) \\ \mathrm{O}(6)-\mathrm{C}(17)-\mathrm{O}(5) & 124.0(4) \\ \mathrm{O}(6)-\mathrm{C}(17)-\mathrm{C}(16) & 126.1(4) \\ \mathrm{O}(5)-\mathrm{C}(17)-\mathrm{C}(16) & 109.8(4) \\ \mathrm{O}(5)-\mathrm{C}(18)-\mathrm{C}(19) & 111.3(3) \\ \mathrm{C}(12)-\mathrm{C}(20)-\mathrm{O}(8) & 126.7(4) \\ \mathrm{O}(7)-\mathrm{C}(21)-\mathrm{O}(8) & 107.1(3) \\ \mathrm{O}(7)-\mathrm{C}(21)-\mathrm{C}(24) & 109.0(3) \\ \mathrm{O}(8)-\mathrm{C}(21)-\mathrm{C}(24) & 111.6(3) \\ \mathrm{O}(7)-\mathrm{C}(22)-\mathrm{C}(23) & 108.8(4) \\ \mathrm{C}(21)-\mathrm{C}(24)-\mathrm{C}(13) & 111.2(3) \\ \mathrm{C}(4)-\mathrm{O}(1)-\mathrm{C}(2) & 122.8(3) \\ \mathrm{C}(6)-\mathrm{O}(2)-\mathrm{C}(2) & 122.6(3) \\ \mathrm{C}(17)-\mathrm{O}(5)-\mathrm{C}(18) & 117.1(3) \\ \mathrm{C}(21)-\mathrm{O}(7)-\mathrm{C}(22) & 114.1(3) \\ \mathrm{C}(20)-\mathrm{O}(8)-\mathrm{C}(21) & 117.6(3) \\ \mathrm{C}(5)-\mathrm{S}(1)-\mathrm{C}(11) & \\ & \\ & \\ & \end{array}$

Symmetry transformations used to generate equivalent atoms: 
Table 4. Anisotropic displacement parameters $\left(\AA^{2} \times 10^{3}\right)$ for sp4. The anisotropic displacement factor exponent takes the form: $-2 \pi^{2}\left[h^{2} a^{* 2} U^{11}+\ldots+2 h k a^{*} b^{*} U^{12}\right]$

\begin{tabular}{|c|c|c|c|c|c|c|}
\hline & $\mathrm{U}^{11}$ & $\mathrm{U}^{22}$ & $\mathrm{U}^{33}$ & $\mathrm{U}^{23}$ & $\mathrm{U}^{13}$ & $\mathrm{U}^{12}$ \\
\hline $\mathrm{C}(1)$ & $106(4)$ & $70(2)$ & 91(3) & $18(2)$ & $4(3)$ & $-4(3)$ \\
\hline $\mathrm{C}(2)$ & 71(3) & $59(2)$ & $62(2)$ & $-2(2)$ & $-2(2)$ & $5(2)$ \\
\hline C(3) & 82(3) & 93(3) & 81(3) & $0(2)$ & $5(2)$ & 20(3) \\
\hline $\mathrm{C}(4)$ & 70(3) & $58(2)$ & $69(2)$ & $6(2)$ & $-2(2)$ & $-2(2)$ \\
\hline$C(5)$ & $58(2)$ & $52(2)$ & $60(2)$ & $8(2)$ & $-3(2)$ & $-1(2)$ \\
\hline C(6) & 70(3) & $62(2)$ & $63(2)$ & $3(2)$ & $0(2)$ & $-5(2)$ \\
\hline $\mathrm{C}(7)$ & 59(2) & $69(2)$ & $72(2)$ & $12(2)$ & $6(2)$ & 2(2) \\
\hline C(8) & $57(2)$ & $63(2)$ & $66(2)$ & $4(2)$ & $11(2)$ & $7(2)$ \\
\hline C(9) & 75(3) & 82(3) & 91(3) & $-3(2)$ & $26(2)$ & $8(2)$ \\
\hline$C(10)$ & $58(2)$ & $56(2)$ & $58(2)$ & $1(2)$ & $5(2)$ & $-1(2)$ \\
\hline $\mathrm{C}(11)$ & $56(2)$ & $62(2)$ & $57(2)$ & $-1(2)$ & $3(2)$ & $-8(2)$ \\
\hline $\mathrm{C}(12)$ & $68(2)$ & $55(2)$ & $60(2)$ & $-1(2)$ & $9(2)$ & $-2(2)$ \\
\hline C(13) & 75(3) & $57(2)$ & $60(2)$ & $4(2)$ & $3(2)$ & $-4(2)$ \\
\hline $\mathrm{C}(14)$ & 78(3) & 63(2) & $60(2)$ & $-2(2)$ & $10(2)$ & $-15(2)$ \\
\hline C(15) & $106(4)$ & 88(3) & 75(3) & $9(2)$ & $-14(3)$ & $-11(3)$ \\
\hline$C(16)$ & 63(2) & $56(2)$ & $60(2)$ & $-6(2)$ & $6(2)$ & $-9(2)$ \\
\hline$C(17)$ & 72(3) & 73(2) & $67(2)$ & $-8(2)$ & $9(2)$ & $-11(2)$ \\
\hline $\mathrm{C}(18)$ & 92(3) & 78(3) & $67(2)$ & $-14(2)$ & $20(2)$ & $0(2)$ \\
\hline C(19) & 131(4) & 72(3) & 93(3) & $-4(2)$ & 5(3) & 19(3) \\
\hline $\mathrm{C}(20)$ & 81(3) & $57(2)$ & $66(2)$ & $-2(2)$ & $11(2)$ & $-8(2)$ \\
\hline $\mathrm{C}(21)$ & 79(3) & $59(2)$ & $65(2)$ & $-4(2)$ & $2(2)$ & $-9(2)$ \\
\hline$C(22)$ & $97(4)$ & $72(2)$ & 81(3) & $-22(2)$ & $-7(3)$ & $-3(2)$ \\
\hline C(23) & $109(4)$ & 99(3) & 91(3) & 6(3) & $-13(3)$ & $-36(3)$ \\
\hline C(24) & 82(3) & $64(2)$ & $62(2)$ & $-7(2)$ & $9(2)$ & $-18(2)$ \\
\hline $\mathrm{O}(1)$ & $96(2)$ & 59(1) & $59(1)$ & $0(1)$ & $-4(1)$ & $4(1)$ \\
\hline $\mathrm{O}(2)$ & $81(2)$ & $58(1)$ & $87(2)$ & 2(1) & $-16(2)$ & $-6(1)$ \\
\hline $\mathrm{O}(3)$ & 143(3) & $67(2)$ & $90(2)$ & $-14(2)$ & $-35(2)$ & $2(2)$ \\
\hline $\mathrm{O}(4)$ & $88(2)$ & 83(2) & $79(2)$ & $3(2)$ & $-21(2)$ & $-16(2)$ \\
\hline O(5) & $74(2)$ & $78(2)$ & $73(2)$ & $-19(1)$ & $15(1)$ & $-8(2)$ \\
\hline $\mathrm{O}(6)$ & $84(2)$ & $125(2)$ & $98(2)$ & $-50(2)$ & $9(2)$ & $-26(2)$ \\
\hline $\mathrm{O}(7)$ & 75(2) & $63(2)$ & $81(2)$ & $-10(1)$ & $4(1)$ & $-9(1)$ \\
\hline $\mathrm{O}(8)$ & $102(2)$ & $75(2)$ & $63(2)$ & $-8(1)$ & $10(2)$ & $-21(2)$ \\
\hline $\mathrm{S}(1)$ & $58(1)$ & $62(1)$ & $86(1)$ & $7(1)$ & $11(1)$ & 2(1) \\
\hline
\end{tabular}


Table 5. Hydrogen coordinates ( x $\left.10^{4}\right)$ and isotropic displacement parameters $\left(\AA^{2} \mathrm{x} 10^{3}\right)$ for sp4.

\begin{tabular}{|c|c|c|c|c|}
\hline & $\mathrm{x}$ & $\mathrm{y}$ & $\mathrm{z}$ & $\mathrm{U}(\mathrm{eq})$ \\
\hline $\mathrm{H}(1 \mathrm{~A})$ & 5790 & 9440 & 4686 & 134 \\
\hline $\mathrm{H}(1 \mathrm{~B})$ & 8270 & 9265 & 4825 & 134 \\
\hline $\mathrm{H}(1 \mathrm{C})$ & 6924 & 9000 & 4131 & 134 \\
\hline $\mathrm{H}(3 \mathrm{~A})$ & 10024 & 9408 & 3252 & 128 \\
\hline $\mathrm{H}(3 \mathrm{~B})$ & 11175 & 9680 & 3981 & 128 \\
\hline $\mathrm{H}(3 \mathrm{C})$ & 10464 & 10075 & 3286 & 128 \\
\hline $\mathrm{H}(7 \mathrm{~A})$ & 4192 & 11382 & 2770 & 80 \\
\hline $\mathrm{H}(7 \mathrm{~B})$ & 3876 & 11342 & 3661 & 80 \\
\hline $\mathrm{H}(9 \mathrm{~A})$ & 5671 & 12261 & 4475 & 123 \\
\hline H(9B) & 3380 & 12300 & 4041 & 123 \\
\hline $\mathrm{H}(9 \mathrm{C})$ & 5190 & 12772 & 3904 & 123 \\
\hline $\mathrm{H}(15 \mathrm{~A})$ & 7120 & 12328 & 606 & 135 \\
\hline $\mathrm{H}(15 \mathrm{~B})$ & 6829 & 12988 & 782 & 135 \\
\hline $\mathrm{H}(15 \mathrm{C})$ & 5870 & 12525 & 1345 & 135 \\
\hline $\mathrm{H}(18 \mathrm{~A})$ & 14834 & 11505 & 122 & 95 \\
\hline $\mathrm{H}(18 \mathrm{~B})$ & 12412 & 11314 & -96 & 95 \\
\hline $\mathrm{H}(19 \mathrm{~A})$ & 15050 & 10726 & 975 & 148 \\
\hline $\mathrm{H}(19 \mathrm{~B})$ & 14746 & 10530 & 116 & 148 \\
\hline $\mathrm{H}(19 \mathrm{C})$ & 12723 & 10522 & 669 & 148 \\
\hline $\mathrm{H}(22 \mathrm{~A})$ & 13541 & 14161 & 4211 & 100 \\
\hline $\mathrm{H}(22 \mathrm{~B})$ & 12526 & 14629 & 3653 & 100 \\
\hline $\mathrm{H}(23 \mathrm{~A})$ & 17039 & 14355 & 3725 & 150 \\
\hline $\mathrm{H}(23 \mathrm{~B})$ & 16021 & 14910 & 4096 & 150 \\
\hline $\mathrm{H}(23 \mathrm{C})$ & 16001 & 14838 & 3199 & 150 \\
\hline $\mathrm{H}(24 \mathrm{~A})$ & 12628 & 13050 & 2318 & 83 \\
\hline $\mathrm{H}(24 \mathrm{~B})$ & 11763 & 13573 & 1824 & 83 \\
\hline $\mathrm{H}(11)$ & $6860(60)$ & 11665(13) & 1919(18) & $58(10)$ \\
\hline $\mathrm{H}(13)$ & $8200(60)$ & 13328(15) & 2045(19) & $73(12)$ \\
\hline $\mathrm{H}(14)$ & $10480(60)$ & $12730(15)$ & 1118(19) & $75(11)$ \\
\hline $\mathrm{H}(16)$ & $11150(50)$ & 12031(12) & 2065(16) & $46(9)$ \\
\hline $\mathrm{H}(20)$ & $9410(50)$ & 12696(13) & 4041(17) & 53(9) \\
\hline $\mathrm{H}(21)$ & $10280(70)$ & $14056(16)$ & $2980(20)$ & $89(12)$ \\
\hline
\end{tabular}


Table 6. Torsion angles $\left[^{\circ}\right]$ for sp4.

\begin{tabular}{|c|c|}
\hline $\mathrm{O}(3)-\mathrm{C}(4)-\mathrm{C}(5)-\mathrm{C}(6)$ & $-158.4(4)$ \\
\hline $\mathrm{O}(1)-\mathrm{C}(4)-\mathrm{C}(5)-\mathrm{C}(6)$ & $17.3(5)$ \\
\hline $\mathrm{O}(3)-\mathrm{C}(4)-\mathrm{C}(5)-\mathrm{C}(7)$ & $-37.4(5)$ \\
\hline $\mathrm{O}(1)-\mathrm{C}(4)-\mathrm{C}(5)-\mathrm{C}(7)$ & 138.2(3) \\
\hline $\mathrm{O}(3)-\mathrm{C}(4)-\mathrm{C}(5)-\mathrm{S}(1)$ & $83.5(5)$ \\
\hline $\mathrm{O}(1)-\mathrm{C}(4)-\mathrm{C}(5)-\mathrm{S}(1)$ & $-100.9(3)$ \\
\hline $\mathrm{C}(4)-\mathrm{C}(5)-\mathrm{C}(6)-\mathrm{O}(4)$ & 158.3(4) \\
\hline $\mathrm{C}(7)-\mathrm{C}(5)-\mathrm{C}(6)-\mathrm{O}(4)$ & $36.3(5)$ \\
\hline $\mathrm{S}(1)-\mathrm{C}(5)-\mathrm{C}(6)-\mathrm{O}(4)$ & $-83.5(4)$ \\
\hline $\mathrm{C}(4)-\mathrm{C}(5)-\mathrm{C}(6)-\mathrm{O}(2)$ & $-19.3(5)$ \\
\hline $\mathrm{C}(7)-\mathrm{C}(5)-\mathrm{C}(6)-\mathrm{O}(2)$ & $-141.3(3)$ \\
\hline $\mathrm{S}(1)-\mathrm{C}(5)-\mathrm{C}(6)-\mathrm{O}(2)$ & $98.9(3)$ \\
\hline $\mathrm{C}(4)-\mathrm{C}(5)-\mathrm{C}(7)-\mathrm{C}(8)$ & $71.2(4)$ \\
\hline $\mathrm{C}(6)-\mathrm{C}(5)-\mathrm{C}(7)-\mathrm{C}(8)$ & $-164.8(3)$ \\
\hline$S(1)-C(5)-C(7)-C(8)$ & $-47.4(4)$ \\
\hline $\mathrm{C}(5)-\mathrm{C}(7)-\mathrm{C}(8)-\mathrm{C}(10)$ & $57.3(4)$ \\
\hline $\mathrm{C}(5)-\mathrm{C}(7)-\mathrm{C}(8)-\mathrm{C}(9)$ & $-121.2(4)$ \\
\hline $\mathrm{C}(9)-\mathrm{C}(8)-\mathrm{C}(10)-\mathrm{C}(12)$ & $5.9(6)$ \\
\hline C(7)-C(8)-C(10)-C(12) & $-172.4(3)$ \\
\hline C(9)-C(8)-C(10)-C(11) & 179.4(4) \\
\hline$C(7)-C(8)-C(10)-C(11)$ & $1.1(5)$ \\
\hline $\mathrm{C}(8)-\mathrm{C}(10)-\mathrm{C}(11)-\mathrm{C}(16)$ & $-177.4(3)$ \\
\hline$C(12)-C(10)-C(11)-C(16)$ & $-3.2(5)$ \\
\hline $\mathrm{C}(8)-\mathrm{C}(10)-\mathrm{C}(11)-\mathrm{S}(1)$ & $-57.4(4)$ \\
\hline $\mathrm{C}(12)-\mathrm{C}(10)-\mathrm{C}(11)-\mathrm{S}(1)$ & 116.8(3) \\
\hline$C(8)-C(10)-C(12)-C(20)$ & $45.0(6)$ \\
\hline$C(11)-C(10)-C(12)-C(20)$ & $-128.6(4)$ \\
\hline $\mathrm{C}(8)-\mathrm{C}(10)-\mathrm{C}(12)-\mathrm{C}(13)$ & $-143.6(4)$ \\
\hline $\mathrm{C}(11)-\mathrm{C}(10)-\mathrm{C}(12)-\mathrm{C}(13)$ & $42.7(5)$ \\
\hline $\mathrm{C}(20)-\mathrm{C}(12)-\mathrm{C}(13)-\mathrm{C}(24)$ & 23.3(5) \\
\hline $\mathrm{C}(10)-\mathrm{C}(12)-\mathrm{C}(13)-\mathrm{C}(24)$ & $-148.2(3)$ \\
\hline$C(20)-C(12)-C(13)-C(14)$ & $147.9(4)$ \\
\hline$C(10)-C(12)-C(13)-C(14)$ & $-23.6(5)$ \\
\hline $\mathrm{C}(12)-\mathrm{C}(13)-\mathrm{C}(14)-\mathrm{C}(15)$ & $95.7(4)$ \\
\hline C(24)-C(13)-C(14)-C(15) & $-141.8(4)$ \\
\hline $\mathrm{C}(12)-\mathrm{C}(13)-\mathrm{C}(14)-\mathrm{C}(16)$ & $-30.3(5)$ \\
\hline $\mathrm{C}(24)-\mathrm{C}(13)-\mathrm{C}(14)-\mathrm{C}(16)$ & $92.2(4)$ \\
\hline $\mathrm{C}(10)-\mathrm{C}(11)-\mathrm{C}(16)-\mathrm{C}(17)$ & $-174.7(3)$ \\
\hline
\end{tabular}




\begin{tabular}{|c|c|}
\hline$S(1)-C(11)-C(16)-C(17)$ & $64.4(4)$ \\
\hline $\mathrm{C}(10)-\mathrm{C}(11)-\mathrm{C}(16)-\mathrm{C}(14)$ & $-50.5(4)$ \\
\hline S(1)-C(11)-C(16)-C(14) & $-171.3(2)$ \\
\hline $\mathrm{C}(15)-\mathrm{C}(14)-\mathrm{C}(16)-\mathrm{C}(17)$ & $68.2(4)$ \\
\hline $\mathrm{C}(13)-\mathrm{C}(14)-\mathrm{C}(16)-\mathrm{C}(17)$ & $-165.9(4)$ \\
\hline $\mathrm{C}(15)-\mathrm{C}(14)-\mathrm{C}(16)-\mathrm{C}(11)$ & $-57.2(4)$ \\
\hline $\mathrm{C}(13)-\mathrm{C}(14)-\mathrm{C}(16)-\mathrm{C}(11)$ & $68.7(4)$ \\
\hline $\mathrm{C}(11)-\mathrm{C}(16)-\mathrm{C}(17)-\mathrm{O}(6)$ & $37.5(6)$ \\
\hline $\mathrm{C}(14)-\mathrm{C}(16)-\mathrm{C}(17)-\mathrm{O}(6)$ & $-86.1(5)$ \\
\hline $\mathrm{C}(11)-\mathrm{C}(16)-\mathrm{C}(17)-\mathrm{O}(5)$ & $-144.9(3)$ \\
\hline C(14)-C(16)-C(17)-O(5) & $91.5(4)$ \\
\hline $\mathrm{C}(10)-\mathrm{C}(12)-\mathrm{C}(20)-\mathrm{O}(8)$ & $175.5(3)$ \\
\hline $\mathrm{C}(13)-\mathrm{C}(12)-\mathrm{C}(20)-\mathrm{O}(8)$ & $4.0(6)$ \\
\hline $\mathrm{O}(7)-\mathrm{C}(21)-\mathrm{C}(24)-\mathrm{C}(13)$ & $173.6(3)$ \\
\hline $\mathrm{O}(8)-\mathrm{C}(21)-\mathrm{C}(24)-\mathrm{C}(13)$ & $55.5(5)$ \\
\hline $\mathrm{C}(12)-\mathrm{C}(13)-\mathrm{C}(24)-\mathrm{C}(21)$ & $-51.9(4)$ \\
\hline $\mathrm{C}(14)-\mathrm{C}(13)-\mathrm{C}(24)-\mathrm{C}(21)$ & $-175.8(3)$ \\
\hline $\mathrm{O}(3)-\mathrm{C}(4)-\mathrm{O}(1)-\mathrm{C}(2)$ & $-171.3(4)$ \\
\hline $\mathrm{C}(5)-\mathrm{C}(4)-\mathrm{O}(1)-\mathrm{C}(2)$ & $12.7(5)$ \\
\hline $\mathrm{O}(2)-\mathrm{C}(2)-\mathrm{O}(1)-\mathrm{C}(4)$ & $-39.0(5)$ \\
\hline $\mathrm{C}(3)-\mathrm{C}(2)-\mathrm{O}(1)-\mathrm{C}(4)$ & 83.3(4) \\
\hline $\mathrm{C}(1)-\mathrm{C}(2)-\mathrm{O}(1)-\mathrm{C}(4)$ & $-152.7(4)$ \\
\hline $\mathrm{O}(4)-\mathrm{C}(6)-\mathrm{O}(2)-\mathrm{C}(2)$ & $173.9(3)$ \\
\hline $\mathrm{C}(5)-\mathrm{C}(6)-\mathrm{O}(2)-\mathrm{C}(2)$ & $-8.4(5)$ \\
\hline $\mathrm{O}(1)-\mathrm{C}(2)-\mathrm{O}(2)-\mathrm{C}(6)$ & $36.6(4)$ \\
\hline $\mathrm{C}(3)-\mathrm{C}(2)-\mathrm{O}(2)-\mathrm{C}(6)$ & $-85.9(4)$ \\
\hline $\mathrm{C}(1)-\mathrm{C}(2)-\mathrm{O}(2)-\mathrm{C}(6)$ & $150.9(3)$ \\
\hline $\mathrm{O}(6)-\mathrm{C}(17)-\mathrm{O}(5)-\mathrm{C}(18)$ & $1.1(6)$ \\
\hline $\mathrm{C}(16)-\mathrm{C}(17)-\mathrm{O}(5)-\mathrm{C}(18)$ & $-176.6(3)$ \\
\hline $\mathrm{C}(19)-\mathrm{C}(18)-\mathrm{O}(5)-\mathrm{C}(17)$ & $-86.8(5)$ \\
\hline $\mathrm{O}(8)-\mathrm{C}(21)-\mathrm{O}(7)-\mathrm{C}(22)$ & $-66.7(4)$ \\
\hline $\mathrm{C}(24)-\mathrm{C}(21)-\mathrm{O}(7)-\mathrm{C}(22)$ & $172.4(3)$ \\
\hline $\mathrm{C}(23)-\mathrm{C}(22)-\mathrm{O}(7)-\mathrm{C}(21)$ & $-167.4(3)$ \\
\hline $\mathrm{C}(12)-\mathrm{C}(20)-\mathrm{O}(8)-\mathrm{C}(21)$ & $-1.9(6)$ \\
\hline $\mathrm{O}(7)-\mathrm{C}(21)-\mathrm{O}(8)-\mathrm{C}(20)$ & $-147.5(3)$ \\
\hline $\mathrm{C}(24)-\mathrm{C}(21)-\mathrm{O}(8)-\mathrm{C}(20)$ & $-28.3(5)$ \\
\hline C(4)-C(5)-S(1)-C(11) & $-125.2(3)$ \\
\hline $\mathrm{C}(6)-\mathrm{C}(5)-\mathrm{S}(1)-\mathrm{C}(11)$ & $113.0(3)$ \\
\hline $\mathrm{C}(7)-\mathrm{C}(5)-\mathrm{S}(1)-\mathrm{C}(11)$ & $-4.9(3)$ \\
\hline C(10)-C(11)-S(1)-C(5) & $53.9(3)$ \\
\hline
\end{tabular}


Symmetry transformations used to generate equivalent atoms: 


\section{Dioxane-4,6-dione 20c (CCDC 600925)}

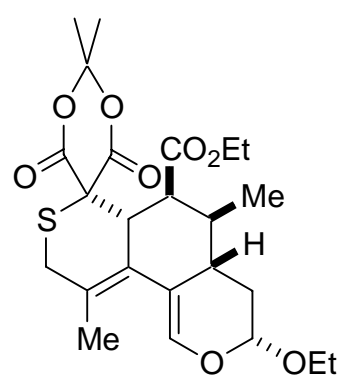

Experimental : The crystals were grown grown by slow evaporation of an ethanol solution at room temperature. One single crystal was mounted using a glass fiber on the goniometer. Data were collected on an Enraf-Nonius CAD-4 automatic diffractometer at the Université de Sherbrooke using $\omega$ scans at 293(2) K. The DIFRAC ${ }^{(1)}$ program was used for centering, indexing, and data collection. Two standard reflections were measured every 100 reflections, 1.1\% intensity decay was observed during data collection. The data were corrected for absorption by empirical methods based on psi scans and reduced with the NRCVAX ${ }^{(2)}$ programs. They were solved using SHELXS-97 $7^{(3)}$ and refined by full-matrix least squares on $F^{2}$ with SHELXL-97 ${ }^{(4)}$. The non-hydrogen atoms were refined anisotropically. The hydrogen atoms were placed at idealized calculated geometric position and refined isotropically using a riding model. The carbon C49 was disordered on two sites and refined with a total occupancy of 1.00 . The refined relative occupancies found were 0.79 and 0.21 respectively for c49 and c49'. Only c49 is shown for clarity.

(1) H.D. Flack, E. Blanc and D. Schwarzenbach (1992), J. Appl. Cryst., 25, 455-459.

(2) E.J. Gabe, Y. Le Page, J.-P. Charland, F.L. Lee, and P.S. White, (1989) J. Appl. Cryst., 22, 384-387.

(3) G. M. Sheldrick, SHELXS-97, G.M. Sheldrick, University of Göttingen, Germany, 1997, Release 97-2.

(4) G. M. Sheldrick, SHELXL-97, G.M. Sheldrick, University of Göttingen, Germany, 1997, Release 97-2. 


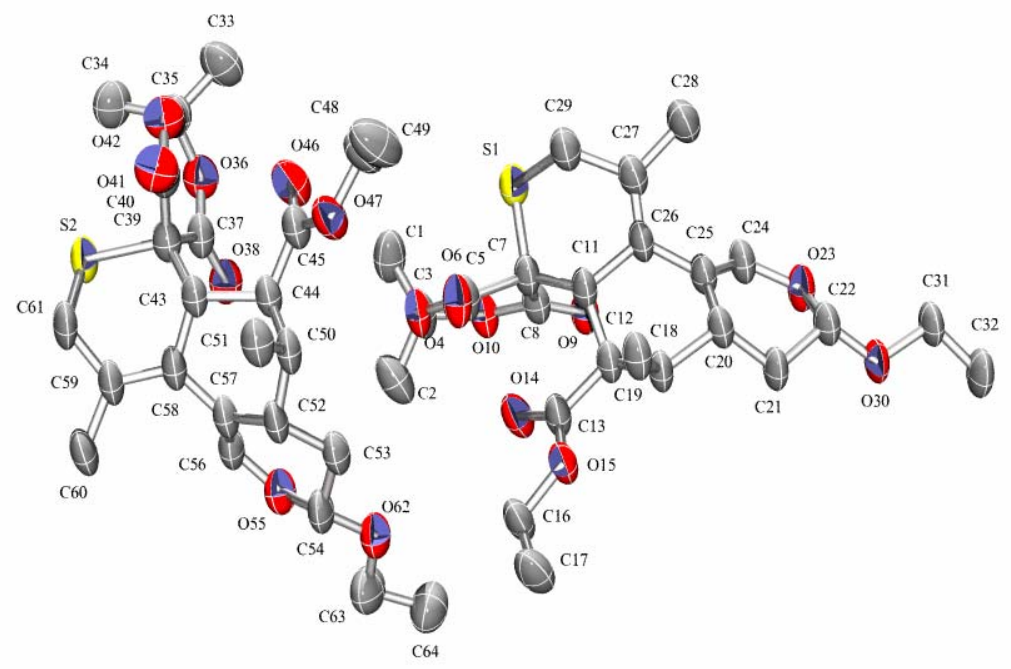

Ellipsoid probability set at 30\%

Table 1. Crystal data and structure refinement for sp5131c1.

Identification code

Empirical formula

Formula weight

Temperature

Wavelength

Crystal system

Space group

Unit cell dimensions

Volume

Z

Density (calculated)

Absorption coefficient

$\mathrm{F}(000)$ sp5131c1

C24 H31 O8 S

479.55

293(2) K

$1.54176 \AA$

Triclinic

P-1

$\mathrm{a}=11.782(8) \AA$

$\alpha=74.73(4)^{\circ}$.

$\mathrm{b}=13.371(6) \AA$

$\beta=70.29(5)^{\circ}$.

c $=17.185(10) \AA$

$\gamma=77.97(5)^{\circ}$.

2437(2) $\AA^{3}$

4

$1.307 \mathrm{Mg} / \mathrm{m}^{3}$

$1.572 \mathrm{~mm}^{-1}$

1020 
Crystal size

Theta range for data collection

Index ranges

Reflections collected

Independent reflections

Completeness to theta $=70.23^{\circ}$

Absorption correction

Max. and min. transmission

Refinement method

Data / restraints / parameters

Goodness-of-fit on $\mathrm{F}^{2}$

Final R indices [I $>2 \operatorname{sigma(I)]~}$

$\mathrm{R}$ indices (all data)

Extinction coefficient

Largest diff. peak and hole
$0.40 \times 0.40 \times 0.35 \mathrm{~mm}^{3}$

2.79 to $70.23^{\circ}$.

$-13<=\mathrm{h}<=14,0<=\mathrm{k}<=16,-19<=\mathrm{l}<=20$

9123

$9123[\mathrm{R}(\mathrm{int})=0.0000]$

$98.4 \%$

Empirical

0.9931 and 0.8818

Full-matrix least-squares on $\mathrm{F}^{2}$

9123 / 0 / 619

0.979

$\mathrm{R} 1=0.0829, \mathrm{wR} 2=0.2279$

$\mathrm{R} 1=0.1468, \mathrm{wR} 2=0.2682$

0.0135(11)

0.288 and -0.447 e. $\AA^{-3}$

Table 2. Atomic coordinates ( $\left.\mathrm{x} 10^{4}\right)$ and equivalent isotropic displacement parameters $\left(\AA^{2} \mathrm{x} 10^{3}\right)$ for sp5131c1. $U(e q)$ is defined as one third of the trace of the orthogonalized $U^{i j}$ tensor.

\begin{tabular}{|c|c|c|c|c|}
\hline & $\mathrm{x}$ & $\mathrm{y}$ & $\mathrm{z}$ & $\mathrm{U}(\mathrm{eq})$ \\
\hline $\mathrm{C}(1)$ & $5675(7)$ & 1158(4) & 643(3) & $145(2)$ \\
\hline$C(2)$ & $3934(5)$ & $105(6)$ & 1461(4) & $149(2)$ \\
\hline$C(3)$ & $5285(5)$ & 153(4) & 1228(3) & $113(2)$ \\
\hline $\mathrm{C}(5)$ & $6427(5)$ & $-608(4)$ & 2261(3) & $104(2)$ \\
\hline $\mathrm{C}(7)$ & 7314(4) & $-1229(3)$ & 1636(3) & $93(1)$ \\
\hline$C(8)$ & $6776(4)$ & $-1437(4)$ & 1028(3) & $96(1)$ \\
\hline $\mathrm{C}(11)$ & 7915(4) & $-2244(3)$ & 2127(3) & 93(1) \\
\hline $\mathrm{C}(12)$ & 7139(4) & $-3154(3)$ & 2517(3) & $92(1)$ \\
\hline$C(13)$ & 5799(4) & $-2822(4)$ & 2927(3) & 101(1) \\
\hline$C(16)$ & $4036(5)$ & $-3134(4)$ & 4075(3) & $124(2)$ \\
\hline $\mathrm{C}(17)$ & $3701(5)$ & $-3819(5)$ & 4906(4) & $144(2)$ \\
\hline $\mathrm{C}(18)$ & $7757(4)$ & $-4040(3)$ & $3077(2)$ & $89(1)$ \\
\hline $\mathrm{C}(19)$ & 7964(5) & $-3704(4)$ & $3800(3)$ & $107(2)$ \\
\hline$C(20)$ & $8974(4)$ & $-4529(3)$ & $2505(3)$ & $95(1)$ \\
\hline$C(21)$ & $8907(4)$ & $-5584(3)$ & 2388(3) & $99(1)$ \\
\hline $\mathrm{C}(22)$ & 10037(4) & $-5937(4)$ & 1732(3) & 101(1) \\
\hline$C(24)$ & 9853(4) & $-4194(4)$ & $940(3)$ & $103(2)$ \\
\hline$C(25)$ & $9378(4)$ & $-3803(3)$ & 1642(3) & $95(1)$ \\
\hline
\end{tabular}




\begin{tabular}{|c|c|c|c|c|}
\hline$C(26)$ & 9189(4) & $-2677(4)$ & 1624(3) & 93(1) \\
\hline $\mathrm{C}(27)$ & 10048(5) & $-2062(4)$ & 1275(3) & $103(2)$ \\
\hline $\mathrm{C}(28)$ & 11375(5) & $-2372(5)$ & $819(4)$ & $130(2)$ \\
\hline C(29) & 9716(5) & $-928(4)$ & 1348(3) & $113(2)$ \\
\hline C(31) & 11081(4) & $-7397(4)$ & 1128(3) & $107(2)$ \\
\hline $\mathrm{O}(4)$ & 5532(3) & $70(2)$ & $2008(2)$ & $113(1)$ \\
\hline $\mathrm{O}(6)$ & 6502(4) & $-627(3)$ & 2939(2) & 122(1) \\
\hline O(9) & 7088(3) & $-2169(2)$ & $701(2)$ & 104(1) \\
\hline $\mathrm{O}(10)$ & $5887(3)$ & $-690(3)$ & $813(2)$ & $112(1)$ \\
\hline $\mathrm{O}(14)$ & $5186(3)$ & $-2176(3)$ & $2560(2)$ & $124(1)$ \\
\hline $\mathrm{O}(15)$ & $5354(3)$ & $-3339(2)$ & $3705(2)$ & $108(1)$ \\
\hline $\mathrm{O}(23)$ & 10101(3) & $-5236(2)$ & $923(2)$ & $112(1)$ \\
\hline $\mathrm{O}(30)$ & 9988(3) & $-6915(2)$ & $1651(2)$ & $110(1)$ \\
\hline$S(1)$ & 8431(1) & $-323(1)$ & $972(1)$ & $110(1)$ \\
\hline C(32) & 10868(6) & $-8463(4)$ & 1165(3) & $129(2)$ \\
\hline C(33) & 6852(6) & 4936(6) & $512(4)$ & $149(2)$ \\
\hline C(34) & 4988(6) & 6251(5) & $837(4)$ & $138(2)$ \\
\hline C(35) & $5613(6)$ & 5171(5) & 1108(3) & $119(2)$ \\
\hline C(37) & 4161(6) & $3853(4)$ & 1779(3) & $104(2)$ \\
\hline C(39) & 3988(5) & 4084(4) & 2627(3) & $94(1)$ \\
\hline $\mathrm{C}(40)$ & $5068(6)$ & $4510(4)$ & 2639(4) & $115(2)$ \\
\hline C(43) & $3693(4)$ & 3137(3) & 3384(3) & $90(1)$ \\
\hline C(44) & $4504(4)$ & 2080(3) & $3258(3)$ & $91(1)$ \\
\hline $\mathrm{C}(45)$ & $5826(5)$ & 2188(4) & 2871(4) & $103(2)$ \\
\hline C(48) & $7836(5)$ & 1631(5) & 2968(5) & $153(3)$ \\
\hline C(49) & 8333(9) & $1340(20)$ & $3671(11)$ & 174(9) \\
\hline$C\left(49^{\prime}\right)$ & $8360(30)$ & $720(40)$ & $3350(30)$ & $111(13)$ \\
\hline$C(50)$ & $4164(4)$ & 1255(3) & 4082(3) & $98(1)$ \\
\hline$C(51)$ & $4245(5)$ & $1557(4)$ & 4854(3) & $115(2)$ \\
\hline C(52) & 2893(4) & $975(3)$ & 4221(3) & $94(1)$ \\
\hline C(53) & 2962(5) & $-25(4)$ & 3931(3) & $103(2)$ \\
\hline C(54) & 1779(5) & $-146(3)$ & 3879(3) & $108(2)$ \\
\hline C(56) & 1538(5) & 1692(4) & 3330(3) & $105(2)$ \\
\hline $\mathrm{C}(57)$ & 2192(4) & 1862(3) & 3756(3) & $90(1)$ \\
\hline C(58) & $2376(4)$ & 2924(3) & 3703(3) & $93(1)$ \\
\hline C(59) & 1492(4) & 3703(4) & 3943(3) & $104(2)$ \\
\hline $\mathrm{C}(60)$ & $167(5)$ & $3579(4)$ & 4358(3) & $121(2)$ \\
\hline C(61) & 1853(5) & 4752(4) & 3810(3) & $116(2)$ \\
\hline $\mathrm{C}(63)$ & $834(7)$ & $-1278(5)$ & $3522(5)$ & $156(3)$ \\
\hline C(64) & $1116(7)$ & $-2252(5)$ & 3196(5) & $162(3)$ \\
\hline
\end{tabular}




\begin{tabular}{lllll}
$\mathrm{O}(36)$ & $4904(4)$ & $4397(3)$ & $1098(2)$ & $119(1)$ \\
$\mathrm{O}(38)$ & $3651(4)$ & $3226(3)$ & $1687(2)$ & $114(1)$ \\
$\mathrm{O}(41)$ & $5309(4)$ & $4456(3)$ & $3286(3)$ & $134(1)$ \\
$\mathrm{O}(42)$ & $5744(4)$ & $5052(3)$ & $1916(2)$ & $122(1)$ \\
$\mathrm{O}(46)$ & $6240(4)$ & $2700(3)$ & $2173(3)$ & $139(1)$ \\
$\mathrm{O}(47)$ & $6517(3)$ & $1634(3)$ & $3336(2)$ & $125(1)$ \\
$\mathrm{O}(55)$ & $1358(3)$ & $705(3)$ & $3289(2)$ & $119(1)$ \\
$\mathrm{O}(62)$ & $1882(4)$ & $-1044(3)$ & $3585(3)$ & $128(1)$ \\
$\mathrm{S}(2)$ & $2747(1)$ & $5181(1)$ & $2725(1)$ & $117(1)$ \\
\hline
\end{tabular}

Table 3. Bond lengths $[\AA]$ and angles $\left[{ }^{\circ}\right]$ for sp5131c1.

\begin{tabular}{ll}
\hline $\mathrm{C}(1)-\mathrm{C}(3)$ & $1.504(7)$ \\
$\mathrm{C}(2)-\mathrm{C}(3)$ & $1.516(8)$ \\
$\mathrm{C}(3)-\mathrm{O}(10)$ & $1.425(5)$ \\
$\mathrm{C}(3)-\mathrm{O}(4)$ & $1.436(6)$ \\
$\mathrm{C}(5)-\mathrm{O}(6)$ & $1.191(5)$ \\
$\mathrm{C}(5)-\mathrm{O}(4)$ & $1.352(5)$ \\
$\mathrm{C}(5)-\mathrm{C}(7)$ & $1.512(6)$ \\
$\mathrm{C}(7)-\mathrm{C}(8)$ & $1.499(7)$ \\
$\mathrm{C}(7)-\mathrm{C}(11)$ & $1.566(6)$ \\
$\mathrm{C}(7)-\mathrm{S}(1)$ & $1.851(5)$ \\
$\mathrm{C}(8)-\mathrm{O}(9)$ & $1.186(5)$ \\
$\mathrm{C}(8)-\mathrm{O}(10)$ & $1.365(5)$ \\
$\mathrm{C}(11)-\mathrm{C}(26)$ & $1.537(5)$ \\
$\mathrm{C}(11)-\mathrm{C}(12)$ & $1.544(7)$ \\
$\mathrm{C}(12)-\mathrm{C}(13)$ & $1.519(6)$ \\
$\mathrm{C}(12)-\mathrm{C}(18)$ & $1.541(5)$ \\
$\mathrm{C}(13)-\mathrm{O}(14)$ & $1.193(5)$ \\
$\mathrm{C}(13)-\mathrm{O}(15)$ & $1.316(5)$ \\
$\mathrm{C}(16)-\mathrm{C}(17)$ & $1.454(7)$ \\
$\mathrm{C}(16)-\mathrm{O}(15)$ & $1.462(6)$ \\
$\mathrm{C}(18)-\mathrm{C}(19)$ & $1.533(6)$ \\
$\mathrm{C}(18)-\mathrm{C}(20)$ & $1.564(5)$ \\
$\mathrm{C}(20)-\mathrm{C}(21)$ & $1.497(6)$ \\
$\mathrm{C}(20)-\mathrm{C}(25)$ & $1.525(6)$ \\
$\mathrm{C}(21)-\mathrm{C}(22)$ & $1.508(5)$ \\
$\mathrm{C}(22)-\mathrm{O}(30)$ & $1.367(5)$ \\
$\mathrm{C}(22)-\mathrm{O}(23)$ & $1.447(5)$ \\
&
\end{tabular}




\begin{tabular}{|c|c|}
\hline C(24)-C(25) & $1.339(6)$ \\
\hline $\mathrm{C}(24)-\mathrm{O}(23)$ & $1.369(5)$ \\
\hline$C(25)-C(26)$ & $1.469(6)$ \\
\hline $\mathrm{C}(26)-\mathrm{C}(27)$ & $1.318(7)$ \\
\hline C(27)-C(29) & $1.513(7)$ \\
\hline$C(27)-C(28)$ & $1.518(7)$ \\
\hline C(29)-S(1) & $1.799(5)$ \\
\hline $\mathrm{C}(31)-\mathrm{O}(30)$ & $1.435(5)$ \\
\hline C(31)-C(32) & $1.479(7)$ \\
\hline C(33)-C(35) & $1.500(8)$ \\
\hline C(34)-C(35) & $1.510(7)$ \\
\hline $\mathrm{C}(35)-\mathrm{O}(42)$ & $1.413(6)$ \\
\hline $\mathrm{C}(35)-\mathrm{O}(36)$ & $1.466(7)$ \\
\hline C(37)-O(38) & $1.197(7)$ \\
\hline $\mathrm{C}(37)-\mathrm{O}(36)$ & $1.338(6)$ \\
\hline C(37)-C(39) & $1.508(7)$ \\
\hline C(39)-C(40) & $1.506(8)$ \\
\hline C(39)-C(43) & $1.558(6)$ \\
\hline C(39)-S(2) & $1.840(4)$ \\
\hline $\mathrm{C}(40)-\mathrm{O}(41)$ & $1.220(6)$ \\
\hline $\mathrm{C}(40)-\mathrm{O}(42)$ & $1.341(7)$ \\
\hline C(43)-C(58) & $1.522(7)$ \\
\hline $\mathrm{C}(43)-\mathrm{C}(44)$ & $1.555(5)$ \\
\hline C(44)-C(45) & $1.494(7)$ \\
\hline $\mathrm{C}(44)-\mathrm{C}(50)$ & $1.536(6)$ \\
\hline $\mathrm{C}(45)-\mathrm{O}(46)$ & $1.208(6)$ \\
\hline $\mathrm{C}(45)-\mathrm{O}(47)$ & $1.313(6)$ \\
\hline $\mathrm{C}(48)-\mathrm{C}\left(49^{\prime}\right)$ & $1.35(4)$ \\
\hline C(48)-C(49) & $1.444(17)$ \\
\hline $\mathrm{C}(48)-\mathrm{O}(47)$ & $1.466(6)$ \\
\hline $\mathrm{C}(50)-\mathrm{C}(51)$ & $1.522(6)$ \\
\hline $\mathrm{C}(50)-\mathrm{C}(52)$ & $1.545(7)$ \\
\hline C(52)-C(57) & $1.505(6)$ \\
\hline C(52)-C(53) & $1.524(6)$ \\
\hline C(53)-C(54) & $1.470(7)$ \\
\hline $\mathrm{C}(54)-\mathrm{O}(62)$ & $1.389(6)$ \\
\hline C(54)-O(55) & $1.437(5)$ \\
\hline $\mathrm{C}(56)-\mathrm{C}(57)$ & $1.313(7)$ \\
\hline $\mathrm{C}(56)-\mathrm{O}(55)$ & $1.403(6)$ \\
\hline C(57)-C(58) & $1.455(7)$ \\
\hline
\end{tabular}




\begin{tabular}{|c|c|}
\hline $\mathrm{C}(58)-\mathrm{C}(59)$ & $1.350(5)$ \\
\hline C(59)-C(61) & $1.487(7)$ \\
\hline C(59)-C(60) & $1.506(7)$ \\
\hline$C(61)-S(2)$ & $1.811(5)$ \\
\hline $\mathrm{C}(63)-\mathrm{O}(62)$ & $1.380(8)$ \\
\hline C(63)-C(64) & 1.482(8) \\
\hline $\mathrm{O}(10)-\mathrm{C}(3)-\mathrm{O}(4)$ & 114.1(3) \\
\hline $\mathrm{O}(10)-\mathrm{C}(3)-\mathrm{C}(1)$ & 108.7(4) \\
\hline $\mathrm{O}(4)-\mathrm{C}(3)-\mathrm{C}(1)$ & 108.3(5) \\
\hline $\mathrm{O}(10)-\mathrm{C}(3)-\mathrm{C}(2)$ & $106.2(5)$ \\
\hline $\mathrm{O}(4)-\mathrm{C}(3)-\mathrm{C}(2)$ & 106.1(4) \\
\hline$C(1)-C(3)-C(2)$ & $113.5(5)$ \\
\hline $\mathrm{O}(6)-\mathrm{C}(5)-\mathrm{O}(4)$ & $117.6(4)$ \\
\hline $\mathrm{O}(6)-\mathrm{C}(5)-\mathrm{C}(7)$ & $124.8(4)$ \\
\hline $\mathrm{O}(4)-\mathrm{C}(5)-\mathrm{C}(7)$ & $117.4(4)$ \\
\hline $\mathrm{C}(8)-\mathrm{C}(7)-\mathrm{C}(5)$ & $113.7(4)$ \\
\hline $\mathrm{C}(8)-\mathrm{C}(7)-\mathrm{C}(11)$ & $113.4(4)$ \\
\hline $\mathrm{C}(5)-\mathrm{C}(7)-\mathrm{C}(11)$ & 109.3(3) \\
\hline $\mathrm{C}(8)-\mathrm{C}(7)-\mathrm{S}(1)$ & 104.3(3) \\
\hline$C(5)-C(7)-S(1)$ & 103.6(3) \\
\hline $\mathrm{C}(11)-\mathrm{C}(7)-\mathrm{S}(1)$ & 112.1(3) \\
\hline $\mathrm{O}(9)-\mathrm{C}(8)-\mathrm{O}(10)$ & $118.2(5)$ \\
\hline $\mathrm{O}(9)-\mathrm{C}(8)-\mathrm{C}(7)$ & $125.6(4)$ \\
\hline $\mathrm{O}(10)-\mathrm{C}(8)-\mathrm{C}(7)$ & 116.1(4) \\
\hline$C(26)-C(11)-C(12)$ & 108.1(3) \\
\hline $\mathrm{C}(26)-\mathrm{C}(11)-\mathrm{C}(7)$ & 114.8(3) \\
\hline $\mathrm{C}(12)-\mathrm{C}(11)-\mathrm{C}(7)$ & $115.4(4)$ \\
\hline$C(13)-C(12)-C(18)$ & $114.9(4)$ \\
\hline$C(13)-C(12)-C(11)$ & $114.4(3)$ \\
\hline $\mathrm{C}(18)-\mathrm{C}(12)-\mathrm{C}(11)$ & $110.0(4)$ \\
\hline $\mathrm{O}(14)-\mathrm{C}(13)-\mathrm{O}(15)$ & $122.7(4)$ \\
\hline $\mathrm{O}(14)-\mathrm{C}(13)-\mathrm{C}(12)$ & $122.6(4)$ \\
\hline $\mathrm{O}(15)-\mathrm{C}(13)-\mathrm{C}(12)$ & $114.6(4)$ \\
\hline $\mathrm{C}(17)-\mathrm{C}(16)-\mathrm{O}(15)$ & 108.1(4) \\
\hline C(19)-C(18)-C(12) & $114.0(3)$ \\
\hline C(19)-C(18)-C(20) & 111.4(4) \\
\hline$C(12)-C(18)-C(20)$ & 109.0(3) \\
\hline C(21)-C(20)-C(25) & 109.4(4) \\
\hline $\mathrm{C}(21)-\mathrm{C}(20)-\mathrm{C}(18)$ & $113.2(4)$ \\
\hline
\end{tabular}




\begin{tabular}{|c|c|}
\hline$C(25)-C(20)-C(18)$ & 111.1(3) \\
\hline$C(20)-C(21)-C(22)$ & $110.4(4)$ \\
\hline $\mathrm{O}(30)-\mathrm{C}(22)-\mathrm{O}(23)$ & $107.7(4)$ \\
\hline $\mathrm{O}(30)-\mathrm{C}(22)-\mathrm{C}(21)$ & $110.0(4)$ \\
\hline $\mathrm{O}(23)-\mathrm{C}(22)-\mathrm{C}(21)$ & 109.3(3) \\
\hline $\mathrm{C}(25)-\mathrm{C}(24)-\mathrm{O}(23)$ & $125.1(4)$ \\
\hline $\mathrm{C}(24)-\mathrm{C}(25)-\mathrm{C}(26)$ & $122.6(4)$ \\
\hline $\mathrm{C}(24)-\mathrm{C}(25)-\mathrm{C}(20)$ & $120.3(4)$ \\
\hline$C(26)-C(25)-C(20)$ & $117.0(4)$ \\
\hline $\mathrm{C}(27)-\mathrm{C}(26)-\mathrm{C}(25)$ & $124.6(4)$ \\
\hline$C(27)-C(26)-C(11)$ & 119.7(4) \\
\hline$C(25)-C(26)-C(11)$ & $115.4(4)$ \\
\hline $\mathrm{C}(26)-\mathrm{C}(27)-\mathrm{C}(29)$ & $118.1(4)$ \\
\hline $\mathrm{C}(26)-\mathrm{C}(27)-\mathrm{C}(28)$ & $127.0(5)$ \\
\hline $\mathrm{C}(29)-\mathrm{C}(27)-\mathrm{C}(28)$ & $114.9(5)$ \\
\hline $\mathrm{C}(27)-\mathrm{C}(29)-\mathrm{S}(1)$ & $111.2(4)$ \\
\hline $\mathrm{O}(30)-\mathrm{C}(31)-\mathrm{C}(32)$ & $107.0(4)$ \\
\hline $\mathrm{C}(5)-\mathrm{O}(4)-\mathrm{C}(3)$ & $124.4(3)$ \\
\hline $\mathrm{C}(8)-\mathrm{O}(10)-\mathrm{C}(3)$ & $125.4(4)$ \\
\hline $\mathrm{C}(13)-\mathrm{O}(15)-\mathrm{C}(16)$ & $115.8(4)$ \\
\hline $\mathrm{C}(24)-\mathrm{O}(23)-\mathrm{C}(22)$ & 115.1(3) \\
\hline $\mathrm{C}(22)-\mathrm{O}(30)-\mathrm{C}(31)$ & $115.4(4)$ \\
\hline C(29)-S(1)-C(7) & $100.2(2)$ \\
\hline $\mathrm{O}(42)-\mathrm{C}(35)-\mathrm{O}(36)$ & $111.7(4)$ \\
\hline $\mathrm{O}(42)-\mathrm{C}(35)-\mathrm{C}(33)$ & $107.7(5)$ \\
\hline $\mathrm{O}(36)-\mathrm{C}(35)-\mathrm{C}(33)$ & $105.2(5)$ \\
\hline $\mathrm{O}(42)-\mathrm{C}(35)-\mathrm{C}(34)$ & $110.3(5)$ \\
\hline $\mathrm{O}(36)-\mathrm{C}(35)-\mathrm{C}(34)$ & $108.7(5)$ \\
\hline C(33)-C(35)-C(34) & $113.2(5)$ \\
\hline $\mathrm{O}(38)-\mathrm{C}(37)-\mathrm{O}(36)$ & $119.0(5)$ \\
\hline $\mathrm{O}(38)-\mathrm{C}(37)-\mathrm{C}(39)$ & $123.3(5)$ \\
\hline $\mathrm{O}(36)-\mathrm{C}(37)-\mathrm{C}(39)$ & $117.8(6)$ \\
\hline C(40)-C(39)-C(37) & $111.9(5)$ \\
\hline $\mathrm{C}(40)-\mathrm{C}(39)-\mathrm{C}(43)$ & $109.2(4)$ \\
\hline C(37)-C(39)-C(43) & $114.3(4)$ \\
\hline $\mathrm{C}(40)-\mathrm{C}(39)-\mathrm{S}(2)$ & 104.8(3) \\
\hline C(37)-C(39)-S(2) & 104.3(3) \\
\hline C(43)-C(39)-S(2) & $111.8(3)$ \\
\hline $\mathrm{O}(41)-\mathrm{C}(40)-\mathrm{O}(42)$ & $117.7(6)$ \\
\hline $\mathrm{O}(41)-\mathrm{C}(40)-\mathrm{C}(39)$ & 123.1(5) \\
\hline
\end{tabular}




\begin{tabular}{|c|c|}
\hline $\mathrm{O}(42)-\mathrm{C}(40)-\mathrm{C}(39)$ & $119.0(5)$ \\
\hline C(58)-C(43)-C(44) & 107.1(4) \\
\hline C(58)-C(43)-C(39) & $115.4(4)$ \\
\hline C(44)-C(43)-C(39) & $115.9(3)$ \\
\hline$C(45)-C(44)-C(50)$ & $116.2(4)$ \\
\hline$C(45)-C(44)-C(43)$ & $113.0(4)$ \\
\hline$C(50)-C(44)-C(43)$ & 109.3(3) \\
\hline $\mathrm{O}(46)-\mathrm{C}(45)-\mathrm{O}(47)$ & $122.3(5)$ \\
\hline $\mathrm{O}(46)-\mathrm{C}(45)-\mathrm{C}(44)$ & $123.2(5)$ \\
\hline $\mathrm{O}(47)-\mathrm{C}(45)-\mathrm{C}(44)$ & $114.4(5)$ \\
\hline C(49')-C(48)-C(49) & $45.5(14)$ \\
\hline $\mathrm{C}\left(49^{\prime}\right)-\mathrm{C}(48)-\mathrm{O}(47)$ & $106.7(16)$ \\
\hline $\mathrm{C}(49)-\mathrm{C}(48)-\mathrm{O}(47)$ & 106.3(7) \\
\hline C(51)-C(50)-C(44) & $114.5(4)$ \\
\hline C(51)-C(50)-C(52) & $111.9(4)$ \\
\hline$C(44)-C(50)-C(52)$ & 109.0(4) \\
\hline C(57)-C(52)-C(53) & $109.4(4)$ \\
\hline C(57)-C(52)-C(50) & $109.8(4)$ \\
\hline C(53)-C(52)-C(50) & 112.3(3) \\
\hline C(54)-C(53)-C(52) & $111.4(4)$ \\
\hline $\mathrm{O}(62)-\mathrm{C}(54)-\mathrm{O}(55)$ & $106.0(4)$ \\
\hline $\mathrm{O}(62)-\mathrm{C}(54)-\mathrm{C}(53)$ & $110.0(4)$ \\
\hline $\mathrm{O}(55)-\mathrm{C}(54)-\mathrm{C}(53)$ & $112.0(4)$ \\
\hline $\mathrm{C}(57)-\mathrm{C}(56)-\mathrm{O}(55)$ & $125.4(4)$ \\
\hline$C(56)-C(57)-C(58)$ & $120.2(4)$ \\
\hline$C(56)-C(57)-C(52)$ & $121.4(5)$ \\
\hline C(58)-C(57)-C(52) & $118.0(5)$ \\
\hline C(59)-C(58)-C(57) & $125.7(5)$ \\
\hline C(59)-C(58)-C(43) & $118.5(4)$ \\
\hline C(57)-C(58)-C(43) & 115.7(3) \\
\hline C(58)-C(59)-C(61) & $117.9(5)$ \\
\hline C(58)-C(59)-C(60) & $124.5(5)$ \\
\hline C(61)-C(59)-C(60) & $117.5(4)$ \\
\hline C(59)-C(61)-S(2) & $111.0(3)$ \\
\hline $\mathrm{O}(62)-\mathrm{C}(63)-\mathrm{C}(64)$ & 109.3(6) \\
\hline $\mathrm{C}(37)-\mathrm{O}(36)-\mathrm{C}(35)$ & $125.5(4)$ \\
\hline $\mathrm{C}(40)-\mathrm{O}(42)-\mathrm{C}(35)$ & $125.2(5)$ \\
\hline $\mathrm{C}(45)-\mathrm{O}(47)-\mathrm{C}(48)$ & $117.5(5)$ \\
\hline $\mathrm{C}(56)-\mathrm{O}(55)-\mathrm{C}(54)$ & 114.1(4) \\
\hline $\mathrm{C}(63)-\mathrm{O}(62)-\mathrm{C}(54)$ & $116.8(5)$ \\
\hline
\end{tabular}


Symmetry transformations used to generate equivalent atoms:

Table 4. Anisotropic displacement parameters $\left(\AA^{2} \times 10^{3}\right)$ for sp5131c1. The anisotropic displacement factor exponent takes the form: $-2 \pi^{2}\left[h^{2} a^{* 2} U^{11}+\ldots+2 h k a^{*} b^{*} U^{12}\right]$

\begin{tabular}{lcccccc}
\hline & $\mathrm{U}^{11}$ & $\mathrm{U}^{22}$ & $\mathrm{U}^{33}$ & $\mathrm{U}^{23}$ & $\mathrm{U}^{13}$ & $\mathrm{U}^{12}$ \\
\hline $\mathrm{C}(1)$ & $190(6)$ & $107(4)$ & $95(3)$ & $-9(3)$ & $-28(4)$ & $34(4)$ \\
$\mathrm{C}(2)$ & $105(4)$ & $194(7)$ & $114(4)$ & $-37(4)$ & $-19(3)$ & $35(4)$ \\
$\mathrm{C}(3)$ & $124(4)$ & $101(3)$ & $84(3)$ & $-24(3)$ & $-23(3)$ & $41(3)$ \\
$\mathrm{C}(5)$ & $112(3)$ & $89(3)$ & $84(3)$ & $-23(2)$ & $-15(3)$ & $32(2)$ \\
$\mathrm{C}(7)$ & $102(3)$ & $75(2)$ & $75(2)$ & $-21(2)$ & $-7(2)$ & $20(2)$ \\
$\mathrm{C}(8)$ & $103(3)$ & $90(3)$ & $70(2)$ & $-19(2)$ & $-15(2)$ & $24(2)$ \\
$\mathrm{C}(11)$ & $97(3)$ & $78(3)$ & $76(2)$ & $-21(2)$ & $-10(2)$ & $25(2)$ \\
$\mathrm{C}(12)$ & $99(3)$ & $80(3)$ & $74(2)$ & $-21(2)$ & $-14(2)$ & $25(2)$ \\
$\mathrm{C}(13)$ & $98(3)$ & $89(3)$ & $87(3)$ & $-23(2)$ & $-16(2)$ & $30(2)$ \\
$\mathrm{C}(16)$ & $89(3)$ & $116(4)$ & $118(4)$ & $-21(3)$ & $3(3)$ & $27(3)$ \\
$\mathrm{C}(17)$ & $110(4)$ & $157(5)$ & $112(4)$ & $-8(4)$ & $4(3)$ & $10(4)$ \\
$\mathrm{C}(18)$ & $96(3)$ & $70(2)$ & $77(2)$ & $-23(2)$ & $-11(2)$ & $24(2)$ \\
$\mathrm{C}(19)$ & $118(4)$ & $101(3)$ & $82(3)$ & $-33(2)$ & $-22(2)$ & $33(3)$ \\
$\mathrm{C}(20)$ & $90(3)$ & $89(3)$ & $80(2)$ & $-23(2)$ & $-14(2)$ & $29(2)$ \\
$\mathrm{C}(21)$ & $111(3)$ & $75(3)$ & $84(3)$ & $-31(2)$ & $-9(2)$ & $27(2)$ \\
$\mathrm{C}(22)$ & $105(3)$ & $89(3)$ & $85(3)$ & $-34(2)$ & $-13(2)$ & $28(2)$ \\
$\mathrm{C}(24)$ & $104(3)$ & $94(3)$ & $90(3)$ & $-35(2)$ & $-19(2)$ & $31(2)$ \\
$\mathrm{C}(25)$ & $89(3)$ & $87(3)$ & $85(3)$ & $-25(2)$ & $-11(2)$ & $22(2)$ \\
$\mathrm{C}(26)$ & $90(3)$ & $79(3)$ & $89(3)$ & $-29(2)$ & $-13(2)$ & $26(2)$ \\
$\mathrm{C}(27)$ & $94(3)$ & $93(3)$ & $92(3)$ & $-20(2)$ & $-9(3)$ & $17(3)$ \\
$\mathrm{C}(28)$ & $101(4)$ & $119(4)$ & $130(4)$ & $-25(3)$ & $-4(3)$ & $16(3)$ \\
$\mathrm{C}(29)$ & $100(3)$ & $104(3)$ & $113(4)$ & $-27(3)$ & $-20(3)$ & $17(3)$ \\
$\mathrm{C}(31)$ & $110(3)$ & $89(3)$ & $95(3)$ & $-41(2)$ & $-10(3)$ & $33(2)$ \\
$\mathrm{O}(4)$ & $125(2)$ & $96(2)$ & $87(2)$ & $-30(2)$ & $-22(2)$ & $49(2)$ \\
$\mathrm{O}(6)$ & $154(3)$ & $108(2)$ & $88(2)$ & $-43(2)$ & $-37(2)$ & $47(2)$ \\
$\mathrm{O}(9)$ & $120(2)$ & $92(2)$ & $85(2)$ & $-36(2)$ & $-19(2)$ & $22(2)$ \\
$\mathrm{O}(10)$ & $120(2)$ & $106(2)$ & $90(2)$ & $-32(2)$ & $-28(2)$ & $38(2)$ \\
$\mathrm{O}(14)$ & $93(2)$ & $124(3)$ & $108(2)$ & $-4(2)$ & $-14(2)$ & $31(2)$ \\
$\mathrm{O}(15)$ & $92(2)$ & $99(2)$ & $93(2)$ & $-14(2)$ & $-5(2)$ & $26(2)$ \\
$\mathrm{O}(23)$ & $136(3)$ & $87(2)$ & $84(2)$ & $-32(2)$ & $-13(2)$ & $31(2)$ \\
& & & & & &
\end{tabular}




\begin{tabular}{|c|c|c|c|c|c|c|}
\hline $\mathrm{O}(30)$ & 109(2) & 85(2) & $106(2)$ & $-42(2)$ & $-3(2)$ & $29(2)$ \\
\hline$S(1)$ & $116(1)$ & $86(1)$ & 99(1) & $-16(1)$ & $-18(1)$ & 19(1) \\
\hline C(32) & $150(5)$ & 90(3) & $116(4)$ & $-46(3)$ & $-10(3)$ & 32(3) \\
\hline C(33) & 121(5) & 180(6) & $111(4)$ & $-22(4)$ & $-7(4)$ & $-4(4)$ \\
\hline C(34) & $165(6)$ & $113(4)$ & $126(4)$ & $-12(3)$ & $-54(4)$ & $6(4)$ \\
\hline C(35) & 143(5) & $110(4)$ & $98(4)$ & $-25(3)$ & $-29(3)$ & $-10(4)$ \\
\hline C(37) & $122(4)$ & 85(3) & 83(3) & $-25(3)$ & $-23(3)$ & 28(3) \\
\hline C(39) & 104(3) & 84(3) & 80(3) & $-24(2)$ & $-22(2)$ & $16(2)$ \\
\hline C(40) & $133(5)$ & 99(4) & 96(4) & $-26(3)$ & $-29(3)$ & 19(3) \\
\hline C(43) & 99(3) & 78(3) & 77(2) & $-28(2)$ & $-21(2)$ & $25(2)$ \\
\hline C(44) & 91(3) & 81(3) & $86(3)$ & $-27(2)$ & $-20(2)$ & $23(2)$ \\
\hline C(45) & $101(4)$ & 91(3) & 104(3) & $-31(3)$ & $-26(3)$ & 25(3) \\
\hline C(48) & 84(4) & 139(5) & 186(7) & $-5(5)$ & $-17(4)$ & 13(3) \\
\hline C(49) & $115(7)$ & 206(18) & $168(11)$ & $11(11)$ & $-44(7)$ & $-9(7)$ \\
\hline$C\left(49^{\prime}\right)$ & $91(18)$ & $120(30)$ & $140(30)$ & $-60(20)$ & $-55(17)$ & $16(16)$ \\
\hline C(50) & 105(3) & 84(3) & $87(3)$ & $-31(2)$ & $-26(2)$ & $39(2)$ \\
\hline C(51) & $127(4)$ & 109(4) & 94(3) & $-29(3)$ & $-34(3)$ & 26(3) \\
\hline C(52) & 94(3) & 80(3) & 82(3) & $-24(2)$ & $-14(2)$ & $33(2)$ \\
\hline C(53) & 98(3) & 90(3) & 95(3) & $-20(2)$ & $-22(2)$ & $29(2)$ \\
\hline C(54) & $114(4)$ & 74(3) & $115(4)$ & $-24(3)$ & $-30(3)$ & $30(2)$ \\
\hline C(56) & $105(3)$ & 80(3) & $105(3)$ & $-26(2)$ & $-21(3)$ & $28(2)$ \\
\hline C(57) & 89(3) & 83(3) & 82(3) & $-26(2)$ & $-21(2)$ & $30(2)$ \\
\hline C(58) & 103(3) & 76(3) & 82(3) & $-28(2)$ & $-20(2)$ & $29(2)$ \\
\hline C(59) & $107(3)$ & 85(3) & $88(3)$ & $-24(2)$ & $-15(2)$ & $36(2)$ \\
\hline C(60) & $107(4)$ & 107(4) & 108(3) & $-28(3)$ & $-9(3)$ & 42(3) \\
\hline C(61) & 133(4) & 85(3) & 105(3) & $-44(3)$ & $-19(3)$ & 39(3) \\
\hline C(63) & $140(6)$ & $110(5)$ & 213(8) & $-29(5)$ & $-73(5)$ & 19(4) \\
\hline C(64) & $176(7)$ & $115(5)$ & 205(7) & $-41(5)$ & $-81(6)$ & $8(4)$ \\
\hline $\mathrm{O}(36)$ & 137(3) & 118(3) & $82(2)$ & $-20(2)$ & $-21(2)$ & $4(2)$ \\
\hline O(38) & 135(3) & 103(3) & $92(2)$ & $-25(2)$ & $-32(2)$ & $9(2)$ \\
\hline $\mathrm{O}(41)$ & $159(4)$ & $146(3)$ & $97(2)$ & $-20(2)$ & $-42(2)$ & $-21(3)$ \\
\hline $\mathrm{O}(42)$ & 136(3) & 126(3) & 92(2) & $-10(2)$ & $-31(2)$ & $-13(2)$ \\
\hline $\mathrm{O}(46)$ & $110(3)$ & 148(3) & 115(3) & $-7(2)$ & $-15(2)$ & $15(2)$ \\
\hline $\mathrm{O}(47)$ & 93(2) & 118(3) & 130(3) & $-9(2)$ & $-23(2)$ & $19(2)$ \\
\hline O(55) & 118(3) & 99(2) & 133(3) & $-23(2)$ & $-52(2)$ & $23(2)$ \\
\hline $\mathrm{O}(62)$ & $119(3)$ & 99(2) & 156(3) & $-33(2)$ & $-47(2)$ & $23(2)$ \\
\hline$S(2)$ & 130(1) & 83(1) & 105(1) & $-23(1)$ & $-19(1)$ & $32(1)$ \\
\hline
\end{tabular}


Table 5. Hydrogen coordinates ( x $\left.10^{4}\right)$ and isotropic displacement parameters $\left(\AA^{2} \mathrm{x} 10^{3}\right)$ for sp5131c1.

\begin{tabular}{|c|c|c|c|c|}
\hline & $\mathrm{x}$ & $\mathrm{y}$ & $\mathrm{z}$ & $\mathrm{U}(\mathrm{eq})$ \\
\hline $\mathrm{H}(1 \mathrm{~A})$ & 5526 & 1222 & 115 & 218 \\
\hline $\mathrm{H}(1 \mathrm{~B})$ & 6527 & 1156 & 546 & 218 \\
\hline $\mathrm{H}(1 \mathrm{C})$ & 5220 & 1738 & 895 & 218 \\
\hline $\mathrm{H}(2 \mathrm{~A})$ & 3735 & -530 & 1865 & 223 \\
\hline $\mathrm{H}(2 \mathrm{~B})$ & 3727 & 120 & 963 & 223 \\
\hline $\mathrm{H}(2 \mathrm{C})$ & 3485 & 694 & 1701 & 223 \\
\hline $\mathrm{H}(11)$ & 8023 & -2044 & 2604 & 111 \\
\hline $\mathrm{H}(12)$ & 7170 & -3441 & 2040 & 110 \\
\hline $\mathrm{H}(16 \mathrm{~A})$ & 3800 & -2408 & 4125 & 149 \\
\hline $\mathrm{H}(16 \mathrm{~B})$ & 3624 & -3268 & 3717 & 149 \\
\hline $\mathrm{H}(17 \mathrm{~A})$ & 4017 & -4530 & 4858 & 217 \\
\hline $\mathrm{H}(17 \mathrm{~B})$ & 2831 & -3756 & 5133 & 217 \\
\hline $\mathrm{H}(17 \mathrm{C})$ & 4033 & -3624 & 5275 & 217 \\
\hline $\mathrm{H}(18)$ & 7220 & -4585 & 3332 & 107 \\
\hline $\mathrm{H}(19 \mathrm{~A})$ & 8550 & -3219 & 3571 & 161 \\
\hline $\mathrm{H}(19 \mathrm{~B})$ & 8261 & -4308 & 4160 & 161 \\
\hline $\mathrm{H}(19 \mathrm{C})$ & 7210 & -3376 & 4122 & 161 \\
\hline $\mathrm{H}(20)$ & 9601 & -4613 & 2782 & 114 \\
\hline $\mathrm{H}(21 \mathrm{~A})$ & 8198 & -5552 & 2212 & 118 \\
\hline $\mathrm{H}(21 \mathrm{~B})$ & 8823 & -6085 & 2921 & 118 \\
\hline $\mathrm{H}(22)$ & 10759 & -5940 & 1895 & 121 \\
\hline $\mathrm{H}(28 \mathrm{~A})$ & 11553 & -3121 & 920 & 194 \\
\hline $\mathrm{H}(28 \mathrm{~B})$ & 11882 & -2096 & 1025 & 194 \\
\hline $\mathrm{H}(28 \mathrm{C})$ & 11529 & -2095 & 223 & 194 \\
\hline $\mathrm{H}(29 \mathrm{~A})$ & 10406 & -554 & 1021 & 135 \\
\hline $\mathrm{H}(29 \mathrm{~B})$ & 9525 & -885 & 1934 & 135 \\
\hline $\mathrm{H}(31 \mathrm{~A})$ & 11761 & -7431 & 1335 & 128 \\
\hline $\mathrm{H}(31 \mathrm{~B})$ & 11262 & -6998 & 551 & 128 \\
\hline $\mathrm{H}(32 \mathrm{~A})$ & 10696 & -8851 & 1738 & 193 \\
\hline $\mathrm{H}(32 \mathrm{~B})$ & 11580 & -8810 & 817 & 193 \\
\hline $\mathrm{H}(32 \mathrm{C})$ & 10189 & -8418 & 962 & 193 \\
\hline $\mathrm{H}(33 \mathrm{~A})$ & 6787 & 4994 & -40 & 224 \\
\hline $\mathrm{H}(33 \mathrm{~B})$ & 7200 & 4238 & 711 & 224 \\
\hline $\mathrm{H}(33 \mathrm{C})$ & 7364 & 5425 & 484 & 224 \\
\hline
\end{tabular}




\begin{tabular}{|c|c|c|c|c|}
\hline $\mathrm{H}(34 \mathrm{~A})$ & 4197 & 6353 & 1236 & 207 \\
\hline $\mathrm{H}(34 \mathrm{~B})$ & 4905 & 6322 & 288 & 207 \\
\hline $\mathrm{H}(34 \mathrm{C})$ & 5463 & 6765 & 816 & 207 \\
\hline $\mathrm{H}(43)$ & 3845 & 3317 & 3854 & 107 \\
\hline $\mathrm{H}(44)$ & 4267 & 1850 & 2845 & 110 \\
\hline $\mathrm{H}(48 \mathrm{~A})$ & 8172 & 1133 & 2601 & 183 \\
\hline $\mathrm{H}(48 \mathrm{~B})$ & 8023 & 2320 & 2641 & 183 \\
\hline $\mathrm{H}(49 \mathrm{~A})$ & 8146 & 652 & 3986 & 262 \\
\hline $\mathrm{H}(49 \mathrm{~B})$ & 7984 & 1831 & 4031 & 262 \\
\hline $\mathrm{H}(49 \mathrm{C})$ & 9200 & 1330 & 3461 & 262 \\
\hline $\mathrm{H}(49 \mathrm{D})$ & 8090 & 681 & 3954 & 167 \\
\hline $\mathrm{H}(49 \mathrm{E})$ & 9228 & 703 & 3149 & 167 \\
\hline $\mathrm{H}(49 \mathrm{~F})$ & 8133 & 145 & 3234 & 167 \\
\hline $\mathrm{H}(50)$ & 4745 & 622 & 3998 & 118 \\
\hline $\mathrm{H}(51 \mathrm{~A})$ & 5012 & 1804 & 4721 & 172 \\
\hline $\mathrm{H}(51 \mathrm{~B})$ & 4180 & 958 & 5313 & 172 \\
\hline $\mathrm{H}(51 \mathrm{C})$ & 3595 & 2100 & 5015 & 172 \\
\hline $\mathrm{H}(52)$ & 2454 & 874 & 4827 & 113 \\
\hline $\mathrm{H}(53 \mathrm{~A})$ & 3563 & -9 & 3380 & 123 \\
\hline $\mathrm{H}(53 \mathrm{~B})$ & 3221 & -622 & 4325 & 123 \\
\hline $\mathrm{H}(54)$ & 1178 & -194 & 4440 & 130 \\
\hline $\mathrm{H}(60 \mathrm{~A})$ & -295 & 3984 & 3989 & 181 \\
\hline $\mathrm{H}(60 \mathrm{~B})$ & -115 & 3818 & 4880 & 181 \\
\hline $\mathrm{H}(60 \mathrm{C})$ & 67 & 2855 & 4471 & 181 \\
\hline $\mathrm{H}(61 \mathrm{~A})$ & 2324 & 4721 & 4184 & 139 \\
\hline $\mathrm{H}(61 \mathrm{~B})$ & 1130 & 5253 & 3949 & 139 \\
\hline $\mathrm{H}(63 \mathrm{~A})$ & 508 & -708 & 3142 & 187 \\
\hline $\mathrm{H}(63 \mathrm{~B})$ & 227 & -1370 & 4072 & 187 \\
\hline $\mathrm{H}(64 \mathrm{~A})$ & 1713 & -2155 & 2649 & 242 \\
\hline $\mathrm{H}(64 \mathrm{~B})$ & 389 & -2416 & 3150 & 242 \\
\hline $\mathrm{H}(64 \mathrm{C})$ & 1430 & -2816 & 3578 & 242 \\
\hline
\end{tabular}


Table 6. Torsion angles $\left[^{\circ}\right]$ for sp5131c1.

\begin{tabular}{|c|c|}
\hline $\mathrm{O}(6)-\mathrm{C}(5)-\mathrm{C}(7)-\mathrm{C}(8)$ & $155.2(5)$ \\
\hline $\mathrm{O}(4)-\mathrm{C}(5)-\mathrm{C}(7)-\mathrm{C}(8)$ & $-29.5(6)$ \\
\hline $\mathrm{O}(6)-\mathrm{C}(5)-\mathrm{C}(7)-\mathrm{C}(11)$ & 27.3(8) \\
\hline $\mathrm{O}(4)-\mathrm{C}(5)-\mathrm{C}(7)-\mathrm{C}(11)$ & $-157.4(5)$ \\
\hline $\mathrm{O}(6)-\mathrm{C}(5)-\mathrm{C}(7)-\mathrm{S}(1)$ & $-92.3(6)$ \\
\hline $\mathrm{O}(4)-\mathrm{C}(5)-\mathrm{C}(7)-\mathrm{S}(1)$ & $83.0(5)$ \\
\hline $\mathrm{C}(5)-\mathrm{C}(7)-\mathrm{C}(8)-\mathrm{O}(9)$ & $-151.3(5)$ \\
\hline $\mathrm{C}(11)-\mathrm{C}(7)-\mathrm{C}(8)-\mathrm{O}(9)$ & $-25.6(6)$ \\
\hline $\mathrm{S}(1)-\mathrm{C}(7)-\mathrm{C}(8)-\mathrm{O}(9)$ & $96.6(5)$ \\
\hline C(5)-C(7)-C(8)-O(10) & $31.5(6)$ \\
\hline C(11)-C(7)-C(8)-O(10) & 157.2(4) \\
\hline $\mathrm{S}(1)-\mathrm{C}(7)-\mathrm{C}(8)-\mathrm{O}(10)$ & $-80.6(4)$ \\
\hline C(8)-C(7)-C(11)-C(26) & $79.0(5)$ \\
\hline C(5)-C(7)-C(11)-C(26) & $-153.0(5)$ \\
\hline S(1)-C(7)-C(11)-C(26) & $-38.8(5)$ \\
\hline C(8)-C(7)-C(11)-C(12) & $-47.8(5)$ \\
\hline$C(5)-C(7)-C(11)-C(12)$ & $80.2(5)$ \\
\hline S(1)-C(7)-C(11)-C(12) & $-165.5(3)$ \\
\hline C(26)-C(11)-C(12)-C(13) & $-171.4(4)$ \\
\hline C(7)-C(11)-C(12)-C(13) & $-41.3(5)$ \\
\hline C(26)-C(11)-C(12)-C(18) & $57.5(4)$ \\
\hline C(7)-C(11)-C(12)-C(18) & $-172.4(3)$ \\
\hline C(18)-C(12)-C(13)-O(14) & $-176.8(5)$ \\
\hline $\mathrm{C}(11)-\mathrm{C}(12)-\mathrm{C}(13)-\mathrm{O}(14)$ & $54.5(7)$ \\
\hline C(18)-C(12)-C(13)-O(15) & $0.0(6)$ \\
\hline C(11)-C(12)-C(13)-O(15) & $-128.7(4)$ \\
\hline $\mathrm{C}(13)-\mathrm{C}(12)-\mathrm{C}(18)-\mathrm{C}(19)$ & $-72.3(6)$ \\
\hline C(11)-C(12)-C(18)-C(19) & $58.6(5)$ \\
\hline C(13)-C(12)-C(18)-C(20) & $162.6(4)$ \\
\hline $\mathrm{C}(11)-\mathrm{C}(12)-\mathrm{C}(18)-\mathrm{C}(20)$ & $-66.5(5)$ \\
\hline$C(19)-C(18)-C(20)-C(21)$ & 127.1(4) \\
\hline C(12)-C(18)-C(20)-C(21) & $-106.3(4)$ \\
\hline C(19)-C(18)-C(20)-C(25) & $-109.3(4)$ \\
\hline $\mathrm{C}(12)-\mathrm{C}(18)-\mathrm{C}(20)-\mathrm{C}(25)$ & $17.3(6)$ \\
\hline C(25)-C(20)-C(21)-C(22) & $47.0(5)$ \\
\hline $\mathrm{C}(18)-\mathrm{C}(20)-\mathrm{C}(21)-\mathrm{C}(22)$ & $171.6(4)$ \\
\hline$C(20)-C(21)-C(22)-O(30)$ & $177.9(4)$ \\
\hline $\mathrm{C}(20)-\mathrm{C}(21)-\mathrm{C}(22)-\mathrm{O}(23)$ & $-64.1(5)$ \\
\hline
\end{tabular}




\begin{tabular}{|c|c|}
\hline $\mathrm{O}(23)-\mathrm{C}(24)-\mathrm{C}(25)-\mathrm{C}(26)$ & $176.8(5)$ \\
\hline $\mathrm{O}(23)-\mathrm{C}(24)-\mathrm{C}(25)-\mathrm{C}(20)$ & $-4.9(8)$ \\
\hline C(21)-C(20)-C(25)-C(24) & $-14.1(6)$ \\
\hline C(18)-C(20)-C(25)-C(24) & $-139.9(5)$ \\
\hline$C(21)-C(20)-C(25)-C(26)$ & $164.3(4)$ \\
\hline$C(18)-C(20)-C(25)-C(26)$ & $38.5(6)$ \\
\hline $\mathrm{C}(24)-\mathrm{C}(25)-\mathrm{C}(26)-\mathrm{C}(27)$ & $-55.9(7)$ \\
\hline$C(20)-C(25)-C(26)-C(27)$ & $125.7(5)$ \\
\hline$C(24)-C(25)-C(26)-C(11)$ & $130.0(5)$ \\
\hline$C(20)-C(25)-C(26)-C(11)$ & $-48.4(6)$ \\
\hline C(12)-C(11)-C(26)-C(27) & $-175.6(4)$ \\
\hline C(7)-C(11)-C(26)-C(27) & $54.0(6)$ \\
\hline $\mathrm{C}(12)-\mathrm{C}(11)-\mathrm{C}(26)-\mathrm{C}(25)$ & $-1.2(5)$ \\
\hline C(7)-C(11)-C(26)-C(25) & $-131.6(4)$ \\
\hline C(25)-C(26)-C(27)-C(29) & $-177.0(4)$ \\
\hline $\mathrm{C}(11)-\mathrm{C}(26)-\mathrm{C}(27)-\mathrm{C}(29)$ & $-3.2(7)$ \\
\hline C(25)-C(26)-C(27)-C(28) & $0.9(9)$ \\
\hline $\mathrm{C}(11)-\mathrm{C}(26)-\mathrm{C}(27)-\mathrm{C}(28)$ & $174.8(5)$ \\
\hline$C(26)-C(27)-C(29)-S(1)$ & $-53.8(6)$ \\
\hline C(28)-C(27)-C(29)-S(1) & $128.0(4)$ \\
\hline $\mathrm{O}(6)-\mathrm{C}(5)-\mathrm{O}(4)-\mathrm{C}(3)$ & $-176.9(5)$ \\
\hline $\mathrm{C}(7)-\mathrm{C}(5)-\mathrm{O}(4)-\mathrm{C}(3)$ & $7.5(8)$ \\
\hline $\mathrm{O}(10)-\mathrm{C}(3)-\mathrm{O}(4)-\mathrm{C}(5)$ & $12.9(7)$ \\
\hline $\mathrm{C}(1)-\mathrm{C}(3)-\mathrm{O}(4)-\mathrm{C}(5)$ & $-108.3(5)$ \\
\hline $\mathrm{C}(2)-\mathrm{C}(3)-\mathrm{O}(4)-\mathrm{C}(5)$ & $129.5(5)$ \\
\hline $\mathrm{O}(9)-\mathrm{C}(8)-\mathrm{O}(10)-\mathrm{C}(3)$ & $170.4(5)$ \\
\hline $\mathrm{C}(7)-\mathrm{C}(8)-\mathrm{O}(10)-\mathrm{C}(3)$ & $-12.2(7)$ \\
\hline $\mathrm{O}(4)-\mathrm{C}(3)-\mathrm{O}(10)-\mathrm{C}(8)$ & $-10.4(7)$ \\
\hline $\mathrm{C}(1)-\mathrm{C}(3)-\mathrm{O}(10)-\mathrm{C}(8)$ & $110.6(6)$ \\
\hline $\mathrm{C}(2)-\mathrm{C}(3)-\mathrm{O}(10)-\mathrm{C}(8)$ & $-126.9(5)$ \\
\hline $\mathrm{O}(14)-\mathrm{C}(13)-\mathrm{O}(15)-\mathrm{C}(16)$ & $4.5(8)$ \\
\hline $\mathrm{C}(12)-\mathrm{C}(13)-\mathrm{O}(15)-\mathrm{C}(16)$ & $-172.3(5)$ \\
\hline $\mathrm{C}(17)-\mathrm{C}(16)-\mathrm{O}(15)-\mathrm{C}(13)$ & $176.2(5)$ \\
\hline $\mathrm{C}(25)-\mathrm{C}(24)-\mathrm{O}(23)-\mathrm{C}(22)$ & $-11.5(7)$ \\
\hline $\mathrm{O}(30)-\mathrm{C}(22)-\mathrm{O}(23)-\mathrm{C}(24)$ & $164.5(4)$ \\
\hline $\mathrm{C}(21)-\mathrm{C}(22)-\mathrm{O}(23)-\mathrm{C}(24)$ & $45.0(6)$ \\
\hline $\mathrm{O}(23)-\mathrm{C}(22)-\mathrm{O}(30)-\mathrm{C}(31)$ & $69.0(5)$ \\
\hline $\mathrm{C}(21)-\mathrm{C}(22)-\mathrm{O}(30)-\mathrm{C}(31)$ & $-172.1(4)$ \\
\hline $\mathrm{C}(32)-\mathrm{C}(31)-\mathrm{O}(30)-\mathrm{C}(22)$ & $176.6(4)$ \\
\hline C(27)-C(29)-S(1)-C(7) & $55.2(4)$ \\
\hline
\end{tabular}




\begin{tabular}{|c|c|}
\hline C(8)-C(7)-S(1)-C(29) & $-133.4(3)$ \\
\hline C(5)-C(7)-S(1)-C(29) & 107.4(3) \\
\hline C(11)-C(7)-S(1)-C(29) & $-10.4(4)$ \\
\hline $\mathrm{O}(38)-\mathrm{C}(37)-\mathrm{C}(39)-\mathrm{C}(40)$ & $155.3(5)$ \\
\hline $\mathrm{O}(36)-\mathrm{C}(37)-\mathrm{C}(39)-\mathrm{C}(40)$ & $-26.3(6)$ \\
\hline $\mathrm{O}(38)-\mathrm{C}(37)-\mathrm{C}(39)-\mathrm{C}(43)$ & $30.5(7)$ \\
\hline $\mathrm{O}(36)-\mathrm{C}(37)-\mathrm{C}(39)-\mathrm{C}(43)$ & $-151.0(4)$ \\
\hline O(38)-C(37)-C(39)-S(2) & $-91.9(5)$ \\
\hline O(36)-C(37)-C(39)-S(2) & $86.5(5)$ \\
\hline $\mathrm{C}(37)-\mathrm{C}(39)-\mathrm{C}(40)-\mathrm{O}(41)$ & $-156.9(5)$ \\
\hline $\mathrm{C}(43)-\mathrm{C}(39)-\mathrm{C}(40)-\mathrm{O}(41)$ & $-29.4(6)$ \\
\hline$S(2)-C(39)-C(40)-O(41)$ & $90.6(5)$ \\
\hline $\mathrm{C}(37)-\mathrm{C}(39)-\mathrm{C}(40)-\mathrm{O}(42)$ & $28.7(6)$ \\
\hline $\mathrm{C}(43)-\mathrm{C}(39)-\mathrm{C}(40)-\mathrm{O}(42)$ & $156.3(4)$ \\
\hline $\mathrm{S}(2)-\mathrm{C}(39)-\mathrm{C}(40)-\mathrm{O}(42)$ & $-83.7(5)$ \\
\hline $\mathrm{C}(40)-\mathrm{C}(39)-\mathrm{C}(43)-\mathrm{C}(58)$ & $153.9(4)$ \\
\hline C(37)-C(39)-C(43)-C(58) & $-79.9(5)$ \\
\hline S(2)-C(39)-C(43)-C(58) & $38.3(5)$ \\
\hline $\mathrm{C}(40)-\mathrm{C}(39)-\mathrm{C}(43)-\mathrm{C}(44)$ & $-79.7(5)$ \\
\hline C(37)-C(39)-C(43)-C(44) & $46.4(6)$ \\
\hline S(2)-C(39)-C(43)-C(44) & $164.7(3)$ \\
\hline $\mathrm{C}(58)-\mathrm{C}(43)-\mathrm{C}(44)-\mathrm{C}(45)$ & $174.9(4)$ \\
\hline C(39)-C(43)-C(44)-C(45) & $44.4(6)$ \\
\hline $\mathrm{C}(58)-\mathrm{C}(43)-\mathrm{C}(44)-\mathrm{C}(50)$ & $-54.0(5)$ \\
\hline $\mathrm{C}(39)-\mathrm{C}(43)-\mathrm{C}(44)-\mathrm{C}(50)$ & $175.6(4)$ \\
\hline $\mathrm{C}(50)-\mathrm{C}(44)-\mathrm{C}(45)-\mathrm{O}(46)$ & $169.8(5)$ \\
\hline $\mathrm{C}(43)-\mathrm{C}(44)-\mathrm{C}(45)-\mathrm{O}(46)$ & $-62.6(6)$ \\
\hline $\mathrm{C}(50)-\mathrm{C}(44)-\mathrm{C}(45)-\mathrm{O}(47)$ & $-6.8(6)$ \\
\hline $\mathrm{C}(43)-\mathrm{C}(44)-\mathrm{C}(45)-\mathrm{O}(47)$ & $120.8(4)$ \\
\hline $\mathrm{C}(45)-\mathrm{C}(44)-\mathrm{C}(50)-\mathrm{C}(51)$ & $73.4(5)$ \\
\hline $\mathrm{C}(43)-\mathrm{C}(44)-\mathrm{C}(50)-\mathrm{C}(51)$ & $-56.0(5)$ \\
\hline $\mathrm{C}(45)-\mathrm{C}(44)-\mathrm{C}(50)-\mathrm{C}(52)$ & $-160.5(4)$ \\
\hline $\mathrm{C}(43)-\mathrm{C}(44)-\mathrm{C}(50)-\mathrm{C}(52)$ & $70.1(5)$ \\
\hline $\mathrm{C}(51)-\mathrm{C}(50)-\mathrm{C}(52)-\mathrm{C}(57)$ & $103.9(4)$ \\
\hline $\mathrm{C}(44)-\mathrm{C}(50)-\mathrm{C}(52)-\mathrm{C}(57)$ & $-23.7(4)$ \\
\hline $\mathrm{C}(51)-\mathrm{C}(50)-\mathrm{C}(52)-\mathrm{C}(53)$ & $-134.1(4)$ \\
\hline $\mathrm{C}(44)-\mathrm{C}(50)-\mathrm{C}(52)-\mathrm{C}(53)$ & $98.2(4)$ \\
\hline C(57)-C(52)-C(53)-C(54) & $-44.4(5)$ \\
\hline $\mathrm{C}(50)-\mathrm{C}(52)-\mathrm{C}(53)-\mathrm{C}(54)$ & $-166.5(4)$ \\
\hline $\mathrm{C}(52)-\mathrm{C}(53)-\mathrm{C}(54)-\mathrm{O}(62)$ & $177.9(4)$ \\
\hline
\end{tabular}




\begin{tabular}{|c|c|}
\hline $\mathrm{C}(52)-\mathrm{C}(53)-\mathrm{C}(54)-\mathrm{O}(55)$ & $60.3(5)$ \\
\hline O(55)-C(56)-C(57)-C(58) & $175.9(4)$ \\
\hline $\mathrm{O}(55)-\mathrm{C}(56)-\mathrm{C}(57)-\mathrm{C}(52)$ & $3.5(7)$ \\
\hline C(53)-C(52)-C(57)-C(56) & $13.9(6)$ \\
\hline C(50)-C(52)-C(57)-C(56) & $137.6(5)$ \\
\hline C(53)-C(52)-C(57)-C(58) & $-158.6(4)$ \\
\hline C(50)-C(52)-C(57)-C(58) & $-34.9(5)$ \\
\hline C(56)-C(57)-C(58)-C(59) & $61.7(6)$ \\
\hline $\mathrm{C}(52)-\mathrm{C}(57)-\mathrm{C}(58)-\mathrm{C}(59)$ & $-125.6(5)$ \\
\hline C(56)-C(57)-C(58)-C(43) & $-121.1(5)$ \\
\hline C(52)-C(57)-C(58)-C(43) & $51.5(5)$ \\
\hline $\mathrm{C}(44)-\mathrm{C}(43)-\mathrm{C}(58)-\mathrm{C}(59)$ & 173.5(4) \\
\hline C(39)-C(43)-C(58)-C(59) & $-55.8(5)$ \\
\hline $\mathrm{C}(44)-\mathrm{C}(43)-\mathrm{C}(58)-\mathrm{C}(57)$ & $-3.9(5)$ \\
\hline C(39)-C(43)-C(58)-C(57) & $126.8(4)$ \\
\hline C(57)-C(58)-C(59)-C(61) & $-177.7(4)$ \\
\hline $\mathrm{C}(43)-\mathrm{C}(58)-\mathrm{C}(59)-\mathrm{C}(61)$ & $5.2(6)$ \\
\hline C(57)-C(58)-C(59)-C(60) & $4.0(8)$ \\
\hline C(43)-C(58)-C(59)-C(60) & $-173.0(4)$ \\
\hline C(58)-C(59)-C(61)-S(2) & $52.8(6)$ \\
\hline C(60)-C(59)-C(61)-S(2) & $-128.9(4)$ \\
\hline $\mathrm{O}(38)-\mathrm{C}(37)-\mathrm{O}(36)-\mathrm{C}(35)$ & $-178.5(5)$ \\
\hline C(39)-C(37)-O(36)-C(35) & $3.0(7)$ \\
\hline $\mathrm{O}(42)-\mathrm{C}(35)-\mathrm{O}(36)-\mathrm{C}(37)$ & 19.1(7) \\
\hline $\mathrm{C}(33)-\mathrm{C}(35)-\mathrm{O}(36)-\mathrm{C}(37)$ & $135.7(5)$ \\
\hline $\mathrm{C}(34)-\mathrm{C}(35)-\mathrm{O}(36)-\mathrm{C}(37)$ & $-102.9(6)$ \\
\hline $\mathrm{O}(41)-\mathrm{C}(40)-\mathrm{O}(42)-\mathrm{C}(35)$ & $178.2(5)$ \\
\hline $\mathrm{C}(39)-\mathrm{C}(40)-\mathrm{O}(42)-\mathrm{C}(35)$ & $-7.2(7)$ \\
\hline $\mathrm{O}(36)-\mathrm{C}(35)-\mathrm{O}(42)-\mathrm{C}(40)$ & $-16.7(7)$ \\
\hline $\mathrm{C}(33)-\mathrm{C}(35)-\mathrm{O}(42)-\mathrm{C}(40)$ & $-131.8(6)$ \\
\hline $\mathrm{C}(34)-\mathrm{C}(35)-\mathrm{O}(42)-\mathrm{C}(40)$ & $104.2(6)$ \\
\hline $\mathrm{O}(46)-\mathrm{C}(45)-\mathrm{O}(47)-\mathrm{C}(48)$ & $-1.6(8)$ \\
\hline $\mathrm{C}(44)-\mathrm{C}(45)-\mathrm{O}(47)-\mathrm{C}(48)$ & $175.0(5)$ \\
\hline $\mathrm{C}\left(49^{\prime}\right)-\mathrm{C}(48)-\mathrm{O}(47)-\mathrm{C}(45)$ & $-154(2)$ \\
\hline $\mathrm{C}(49)-\mathrm{C}(48)-\mathrm{O}(47)-\mathrm{C}(45)$ & $158.4(13)$ \\
\hline C(57)-C(56)-O(55)-C(54) & $10.4(7)$ \\
\hline $\mathrm{O}(62)-\mathrm{C}(54)-\mathrm{O}(55)-\mathrm{C}(56)$ & $-162.0(4)$ \\
\hline C(53)-C(54)-O(55)-C(56) & $-42.0(5)$ \\
\hline $\mathrm{C}(64)-\mathrm{C}(63)-\mathrm{O}(62)-\mathrm{C}(54)$ & $179.7(5)$ \\
\hline $\mathrm{O}(55)-\mathrm{C}(54)-\mathrm{O}(62)-\mathrm{C}(63)$ & $-60.2(6)$ \\
\hline
\end{tabular}


$\mathrm{C}(53)-\mathrm{C}(54)-\mathrm{O}(62)-\mathrm{C}(63)$

C(59)-C(61)-S(2)-C(39)

C(40)-C(39)-S(2)-C(61)

C(37)-C(39)-S(2)-C(61)

C(43)-C(39)-S(2)-C(61)
178.5(5)

$-56.8(4)$

$-106.0(4)$

136.2(4)

12.2(4)

Symmetry transformations used to generate equivalent atoms: 


\section{Dioxane-4,6-dione 21 (CCDC 600924)}

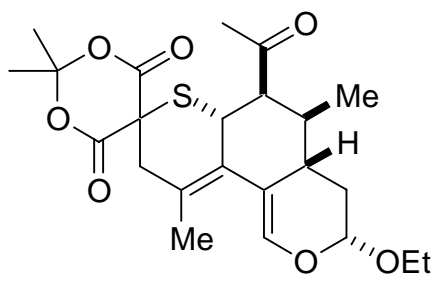

Experimental : The crystals were grown by slow evaporation of an ethanol solution at room temperature. One single crystal of 0.3 X 0.3 X $0.3 \mathrm{~mm}$ was mounted using a glass fiber on the goniometer at 293(2) K. Data were collected on an Enraf-Nonius CAD-4 automatic diffractometer at the Université de Sherbrooke using $\omega / 2$ theta scans. The DIFRAC $^{(1)}$ program was used for centering, indexing, and data collection. Two standard reflections were measured every 100 reflections, no intensity decay was observed during data collection. The data were corrected for absorption by empirical methods based on psi scans and reduced with the NRCVAX ${ }^{(2)}$ programs. They were solved using SHELXS- $97^{(3)}$ and refined by full-matrix least squares on $\mathrm{F}^{2}$ with SHELXL-97 $7^{(4)}$. The non-hydrogen atoms were refined anisotropically. The hydrogen atoms were placed at idealized calculated geometric position and refined isotropically using a riding model. The final absolute structure ${ }^{(5)}$ could not be assigned, the twin BASF parameter was 0.46 for the actual isomer and 0.54 for the inverted structure.

(1) H.D. Flack, E. Blanc and D. Schwarzenbach (1992), J. Appl. Cryst., 25, 455-459.

(2) E.J. Gabe, Y. Le Page, J.-P. Charland, F.L. Lee, and P.S. White, (1989) J. Appl. Cryst., 22, 384-387.

(3) G. M. Sheldrick, SHELXS-97, G.M. Sheldrick, University of Göttingen, Germany, 1997, Release 97-2.

(4) G. M. Sheldrick, SHELXL-97, G.M. Sheldrick, University of Göttingen, Germany, 1997, Release 97-2.

(5) Flack H D (1983), Acta Cryst. A39, 876-881. 


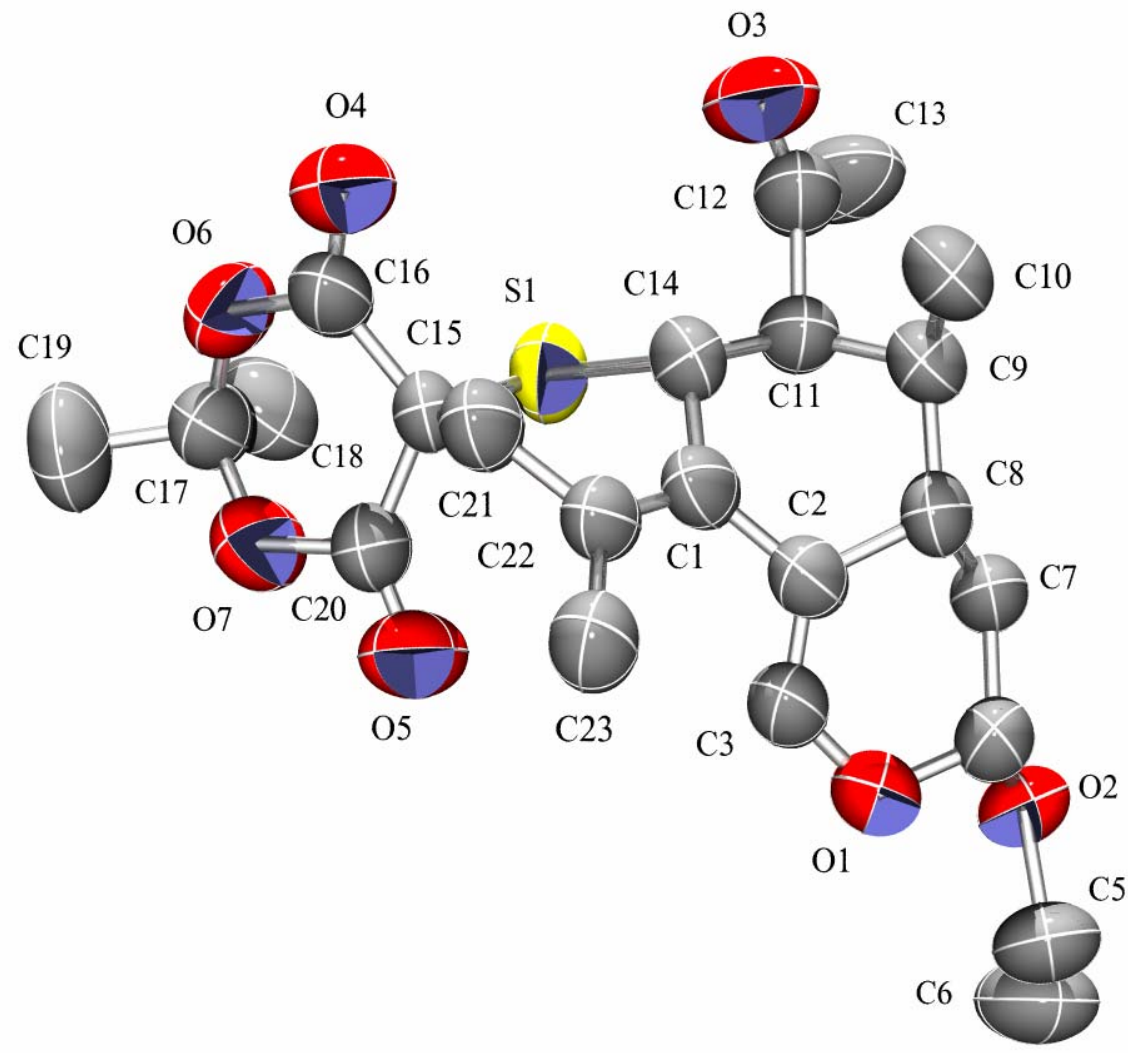

Ellipsoid probability set at 50\%.

Table 1. Crystal data and structure refinement for sp5036.

Identification code

Empirical formula

Formula weight

Temperature

Wavelength

Crystal system

Space group

Unit cell dimensions

Volume

Z

Density (calculated)

Absorption coefficient sp5036

C23 H30 O7 S

450.53

293(2) K

$1.54176 \AA$

Orthorhombic

P212121

$\mathrm{a}=6.186(6) \AA$

$\alpha=90^{\circ}$.

$\mathrm{b}=14.484(15) \AA$

$\beta=90^{\circ}$.

c $=25.998(4) \AA$

$\gamma=90^{\circ}$.

4

$1.285 \mathrm{Mg} / \mathrm{m}^{3}$

$1.577 \mathrm{~mm}^{-1}$ 
$\mathrm{F}(000)$

Crystal size

Theta range for data collection

Index ranges

Reflections collected

Independent reflections

Completeness to theta $=69.88^{\circ}$

Absorption correction

Max. and min. transmission

Refinement method

Data / restraints / parameters

Goodness-of-fit on $\mathrm{F}^{2}$

Final R indices [I $>2 \operatorname{sigma}(\mathrm{I})]$

$\mathrm{R}$ indices (all data)

Absolute structure parameter

Extinction coefficient

Largest diff. peak and hole
960

$0.30 \times 0.30 \times 0.30 \mathrm{~mm}^{3}$

3.40 to $69.88^{\circ}$.

$-7<=\mathrm{h}<=1,-17<=\mathrm{k}<=0,-31<=\mathrm{l}<=0$

2570

2553 [R(int) $=0.0326]$

$97.5 \%$

Empirical

0.6490 and 0.6490

Full-matrix least-squares on $\mathrm{F}^{2}$

2553 / 0 / 288

0.944

$\mathrm{R} 1=0.0710, \mathrm{wR} 2=0.1842$

$\mathrm{R} 1=0.1244, \mathrm{wR} 2=0.2225$

$0.46(7)$

$0.0134(15)$

0.266 and -0.365 e. $\AA^{-3}$

Table 2. Atomic coordinates ( $\left.\mathrm{x} 10^{4}\right)$ and equivalent isotropic displacement parameters $\left(\AA^{2} \mathrm{x} 10^{3}\right)$ for sp5036. $\mathrm{U}(\mathrm{eq})$ is defined as one third of the trace of the orthogonalized $\mathrm{U}^{\mathrm{ij}}$ tensor.

\begin{tabular}{|c|c|c|c|c|}
\hline & $\mathrm{X}$ & $\mathrm{y}$ & $\mathrm{z}$ & $\mathrm{U}(\mathrm{eq})$ \\
\hline $\mathrm{C}(1)$ & $-1591(15)$ & $-598(4)$ & $1449(2)$ & $94(2)$ \\
\hline$C(2)$ & $-391(14)$ & $-1462(4)$ & $1375(2)$ & $88(2)$ \\
\hline $\mathrm{C}(3)$ & $326(17)$ & $-1688(4)$ & $923(2)$ & $100(2)$ \\
\hline$C(4)$ & $2467(17)$ & $-2956(4)$ & 1234(3) & $102(2)$ \\
\hline$C(5)$ & $4660(20)$ & $-3821(6)$ & 671(3) & $139(4)$ \\
\hline$C(6)$ & $6810(30)$ & $-3810(9)$ & $470(4)$ & $175(5)$ \\
\hline$C(7)$ & 2503(17) & $-2428(4)$ & $1725(2)$ & 105(3) \\
\hline $\mathrm{C}(8)$ & $321(15)$ & $-2014(4)$ & $1843(2)$ & $90(2)$ \\
\hline $\mathrm{C}(9)$ & 294(17) & $-1442(5)$ & 2342(2) & $101(2)$ \\
\hline $\mathrm{C}(10)$ & $-2013(18)$ & $-1514(6)$ & 2599(3) & 123(3) \\
\hline $\mathrm{C}(11)$ & $933(16)$ & $-429(4)$ & $2219(2)$ & $94(2)$ \\
\hline$C(12)$ & $1180(20)$ & 101(5) & 2719(3) & $114(3)$ \\
\hline $\mathrm{C}(13)$ & $3470(20)$ & $111(7)$ & $2947(4)$ & $154(4)$ \\
\hline$C(14)$ & $-767(16)$ & $22(4)$ & $1877(2)$ & $91(2)$ \\
\hline$C(15)$ & $-1772(13)$ & $1434(4)$ & $1188(2)$ & $83(2)$ \\
\hline C(16) & $-2516(19)$ & $2372(5)$ & $1386(2)$ & 106(3) \\
\hline
\end{tabular}




\begin{tabular}{lrrrr}
$\mathrm{C}(17)$ & $-160(20)$ & $3095(5)$ & $751(3)$ & $112(3)$ \\
$\mathrm{C}(18)$ & $2050(20)$ & $3133(8)$ & $949(4)$ & $142(4)$ \\
$\mathrm{C}(19)$ & $-740(20)$ & $3898(5)$ & $395(4)$ & $156(4)$ \\
$\mathrm{C}(20)$ & $-873(18)$ & $1456(5)$ & $641(3)$ & $111(3)$ \\
$\mathrm{C}(21)$ & $-3715(16)$ & $753(4)$ & $1207(3)$ & $98(2)$ \\
$\mathrm{C}(22)$ & $-3090(15)$ & $-240(4)$ & $1132(2)$ & $89(2)$ \\
$\mathrm{C}(23)$ & $-4216(16)$ & $-737(5)$ & $707(3)$ & $105(2)$ \\
$\mathrm{O}(1)$ & $1637(11)$ & $-2429(3)$ & $813(2)$ & $112(2)$ \\
$\mathrm{O}(2)$ & $4546(13)$ & $-3214(3)$ & $1097(2)$ & $118(2)$ \\
$\mathrm{O}(3)$ & $-233(13)$ & $504(4)$ & $2926(2)$ & $144(3)$ \\
$\mathrm{O}(4)$ & $-3741(11)$ & $2467(3)$ & $1733(2)$ & $118(2)$ \\
$\mathrm{O}(5)$ & $-774(15)$ & $816(4)$ & $364(2)$ & $137(2)$ \\
$\mathrm{O}(6)$ & $-1695(13)$ & $3130(3)$ & $1160(2)$ & $131(2)$ \\
$\mathrm{O}(7)$ & $-479(14)$ & $2289(3)$ & $438(2)$ & $135(2)$ \\
$\mathrm{S}(1)$ & $454(4)$ & $1060(1)$ & $1598(1)$ & $104(1)$ \\
\hline
\end{tabular}

Table 3. Bond lengths $[\AA]$ and angles $\left[^{\circ}\right]$ for sp5036.

\begin{tabular}{ll}
\hline $\mathrm{C}(1)-\mathrm{C}(22)$ & $1.345(11)$ \\
$\mathrm{C}(1)-\mathrm{C}(2)$ & $1.467(10)$ \\
$\mathrm{C}(1)-\mathrm{C}(14)$ & $1.517(9)$ \\
$\mathrm{C}(2)-\mathrm{C}(3)$ & $1.300(9)$ \\
$\mathrm{C}(2)-\mathrm{C}(8)$ & $1.521(8)$ \\
$\mathrm{C}(3)-\mathrm{O}(1)$ & $1.375(9)$ \\
$\mathrm{C}(4)-\mathrm{O}(2)$ & $1.386(11)$ \\
$\mathrm{C}(4)-\mathrm{O}(1)$ & $1.430(8)$ \\
$\mathrm{C}(4)-\mathrm{C}(7)$ & $1.488(8)$ \\
$\mathrm{C}(5)-\mathrm{O}(2)$ & $1.415(9)$ \\
$\mathrm{C}(5)-\mathrm{C}(6)$ & $1.432(17)$ \\
$\mathrm{C}(7)-\mathrm{C}(8)$ & $1.509(12)$ \\
$\mathrm{C}(8)-\mathrm{C}(9)$ & $1.539(8)$ \\
$\mathrm{C}(9)-\mathrm{C}(11)$ & $1.552(9)$ \\
$\mathrm{C}(9)-\mathrm{C}(10)$ & $1.579(14)$ \\
$\mathrm{C}(11)-\mathrm{C}(12)$ & $1.517(8)$ \\
$\mathrm{C}(11)-\mathrm{C}(14)$ & $1.525(11)$ \\
$\mathrm{C}(12)-\mathrm{O}(3)$ & $1.183(11)$ \\
$\mathrm{C}(12)-\mathrm{C}(13)$ & $1.533(15)$ \\
$\mathrm{C}(14)-\mathrm{S}(1)$ & $1.832(7)$ \\
$\mathrm{C}(15)-\mathrm{C}(16)$ & $1.525(9)$ \\
\end{tabular}




\begin{tabular}{|c|c|}
\hline C(15)-C(20) & $1.526(9)$ \\
\hline C(15)-C(21) & $1.556(11)$ \\
\hline $\mathrm{C}(15)-\mathrm{S}(1)$ & $1.824(7)$ \\
\hline $\mathrm{C}(16)-\mathrm{O}(4)$ & $1.186(10)$ \\
\hline $\mathrm{C}(16)-\mathrm{O}(6)$ & $1.344(9)$ \\
\hline $\mathrm{C}(17)-\mathrm{O}(6)$ & $1.429(11)$ \\
\hline $\mathrm{C}(17)-\mathrm{O}(7)$ & $1.437(9)$ \\
\hline C(17)-C(18) & $1.459(14)$ \\
\hline C(17)-C(19) & $1.528(11)$ \\
\hline $\mathrm{C}(20)-\mathrm{O}(5)$ & $1.175(8)$ \\
\hline $\mathrm{C}(20)-\mathrm{O}(7)$ & $1.340(8)$ \\
\hline C(21)-C(22) & $1.502(9)$ \\
\hline C(22)-C(23) & $1.492(10)$ \\
\hline$C(22)-C(1)-C(2)$ & $126.7(6)$ \\
\hline$C(22)-C(1)-C(14)$ & $116.9(6)$ \\
\hline $\mathrm{C}(2)-\mathrm{C}(1)-\mathrm{C}(14)$ & 115.5(7) \\
\hline $\mathrm{C}(3)-\mathrm{C}(2)-\mathrm{C}(1)$ & $120.4(6)$ \\
\hline C(3)-C(2)-C(8) & 119.6(7) \\
\hline C(1)-C(2)-C(8) & $119.4(5)$ \\
\hline $\mathrm{C}(2)-\mathrm{C}(3)-\mathrm{O}(1)$ & $125.9(6)$ \\
\hline $\mathrm{O}(2)-\mathrm{C}(4)-\mathrm{O}(1)$ & 106.3(6) \\
\hline $\mathrm{O}(2)-\mathrm{C}(4)-\mathrm{C}(7)$ & 110.2(7) \\
\hline $\mathrm{O}(1)-\mathrm{C}(4)-\mathrm{C}(7)$ & $112.8(5)$ \\
\hline $\mathrm{O}(2)-\mathrm{C}(5)-\mathrm{C}(6)$ & 108.9(10) \\
\hline C(4)-C(7)-C(8) & $111.5(7)$ \\
\hline$C(7)-C(8)-C(2)$ & 107.7(6) \\
\hline $\mathrm{C}(7)-\mathrm{C}(8)-\mathrm{C}(9)$ & $113.3(7)$ \\
\hline C(2)-C(8)-C(9) & $112.8(5)$ \\
\hline C(8)-C(9)-C(11) & $109.4(5)$ \\
\hline $\mathrm{C}(8)-\mathrm{C}(9)-\mathrm{C}(10)$ & 109.3(8) \\
\hline C(11)-C(9)-C(10) & $112.3(7)$ \\
\hline $\mathrm{C}(12)-\mathrm{C}(11)-\mathrm{C}(14)$ & $110.7(6)$ \\
\hline C(12)-C(11)-C(9) & 109.1(5) \\
\hline C(14)-C(11)-C(9) & $110.5(7)$ \\
\hline $\mathrm{O}(3)-\mathrm{C}(12)-\mathrm{C}(11)$ & $124.4(10)$ \\
\hline $\mathrm{O}(3)-\mathrm{C}(12)-\mathrm{C}(13)$ & 120.1(7) \\
\hline $\mathrm{C}(11)-\mathrm{C}(12)-\mathrm{C}(13)$ & 115.5(9) \\
\hline $\mathrm{C}(1)-\mathrm{C}(14)-\mathrm{C}(11)$ & $113.9(5)$ \\
\hline $\mathrm{C}(1)-\mathrm{C}(14)-\mathrm{S}(1)$ & $109.6(4)$ \\
\hline
\end{tabular}




\begin{tabular}{|c|c|}
\hline $\mathrm{C}(11)-\mathrm{C}(14)-\mathrm{S}(1)$ & $107.3(6)$ \\
\hline$C(16)-C(15)-C(20)$ & $113.9(5)$ \\
\hline $\mathrm{C}(16)-\mathrm{C}(15)-\mathrm{C}(21)$ & $108.7(7)$ \\
\hline C(20)-C(15)-C(21) & 109.0(6) \\
\hline $\mathrm{C}(16)-\mathrm{C}(15)-\mathrm{S}(1)$ & 107.1(5) \\
\hline$C(20)-C(15)-S(1)$ & $106.0(6)$ \\
\hline $\mathrm{C}(21)-\mathrm{C}(15)-\mathrm{S}(1)$ & $112.1(4)$ \\
\hline $\mathrm{O}(4)-\mathrm{C}(16)-\mathrm{O}(6)$ & $118.5(7)$ \\
\hline $\mathrm{O}(4)-\mathrm{C}(16)-\mathrm{C}(15)$ & $123.6(7)$ \\
\hline $\mathrm{O}(6)-\mathrm{C}(16)-\mathrm{C}(15)$ & $117.9(7)$ \\
\hline $\mathrm{O}(6)-\mathrm{C}(17)-\mathrm{O}(7)$ & $111.0(8)$ \\
\hline $\mathrm{O}(6)-\mathrm{C}(17)-\mathrm{C}(18)$ & $110.9(7)$ \\
\hline $\mathrm{O}(7)-\mathrm{C}(17)-\mathrm{C}(18)$ & 111.1(9) \\
\hline $\mathrm{O}(6)-\mathrm{C}(17)-\mathrm{C}(19)$ & $105.5(8)$ \\
\hline $\mathrm{O}(7)-\mathrm{C}(17)-\mathrm{C}(19)$ & 104.1(7) \\
\hline C(18)-C(17)-C(19) & $113.9(9)$ \\
\hline $\mathrm{O}(5)-\mathrm{C}(20)-\mathrm{O}(7)$ & $117.4(6)$ \\
\hline $\mathrm{O}(5)-\mathrm{C}(20)-\mathrm{C}(15)$ & $124.9(7)$ \\
\hline $\mathrm{O}(7)-\mathrm{C}(20)-\mathrm{C}(15)$ & $116.9(6)$ \\
\hline C(22)-C(21)-C(15) & $113.8(7)$ \\
\hline $\mathrm{C}(1)-\mathrm{C}(22)-\mathrm{C}(23)$ & $126.2(6)$ \\
\hline $\mathrm{C}(1)-\mathrm{C}(22)-\mathrm{C}(21)$ & $117.9(6)$ \\
\hline C(23)-C(22)-C(21) & $116.0(7)$ \\
\hline $\mathrm{C}(3)-\mathrm{O}(1)-\mathrm{C}(4)$ & $118.0(5)$ \\
\hline $\mathrm{C}(4)-\mathrm{O}(2)-\mathrm{C}(5)$ & $114.5(8)$ \\
\hline $\mathrm{C}(16)-\mathrm{O}(6)-\mathrm{C}(17)$ & $123.2(6)$ \\
\hline $\mathrm{C}(20)-\mathrm{O}(7)-\mathrm{C}(17)$ & $122.3(5)$ \\
\hline$C(15)-S(1)-C(14)$ & 99.4(4) \\
\hline
\end{tabular}

Symmetry transformations used to generate equivalent atoms:

Table 4. Anisotropic displacement parameters $\left(\AA^{2} \mathrm{x} 10^{3}\right)$ for sp5036. The anisotropic displacement factor exponent takes the form: $-2 \pi^{2}\left[h^{2} a^{* 2} U^{11}+\ldots+2 h k a^{*} b^{*} U^{12}\right]$

\begin{tabular}{lcccccc}
\hline & $\mathrm{U}^{11}$ & $\mathrm{U}^{22}$ & $\mathrm{U}^{33}$ & $\mathrm{U}^{23}$ & $\mathrm{U}^{13}$ & \multirow{2}{*}{$\mathrm{U}^{12}$} \\
\hline $\mathrm{C}(1)$ & $150(7)$ & $68(3)$ & $65(3)$ & $3(3)$ & $2(4)$ & $-13(4)$ \\
$\mathrm{C}(2)$ & $125(6)$ & $75(3)$ & $65(3)$ & $1(3)$ & $0(4)$ & $-8(4)$ \\
$\mathrm{C}(3)$ & $150(7)$ & $78(3)$ & $71(4)$ & $-1(3)$ & $-9(4)$ & $0(5)$ \\
& & & & & &
\end{tabular}




\begin{tabular}{|c|c|c|c|c|c|c|}
\hline C(4) & 152(8) & 72(3) & 81(4) & $-3(3)$ & $-7(5)$ & $6(5)$ \\
\hline C(5) & 200(11) & $117(6)$ & $100(5)$ & $-37(5)$ & $0(7)$ & $9(8)$ \\
\hline C(6) & $232(14)$ & 182(10) & 109(7) & $-48(7)$ & 2(9) & $22(11)$ \\
\hline $\mathrm{C}(7)$ & $175(8)$ & 72(3) & 69(3) & $-5(3)$ & $-4(5)$ & $14(5)$ \\
\hline C(8) & 132(7) & $66(3)$ & 72(3) & $9(3)$ & $6(4)$ & 1(4) \\
\hline C(9) & $150(7)$ & $86(4)$ & 68(3) & 7(3) & 13(5) & 7(5) \\
\hline C(10) & 178(9) & $116(5)$ & 77(4) & $14(4)$ & 13(6) & 22(7) \\
\hline C(11) & 139(7) & 78(3) & 66(3) & $-10(3)$ & $-12(4)$ & $15(5)$ \\
\hline $\mathrm{C}(12)$ & $175(10)$ & 88(4) & 80(4) & $-13(3)$ & $-16(6)$ & 10(6) \\
\hline C(13) & 181(11) & 162(8) & $120(6)$ & $-67(6)$ & $-30(7)$ & 17(8) \\
\hline C(14) & $121(6)$ & 82(3) & 70(3) & $5(3)$ & $-6(4)$ & $15(4)$ \\
\hline C(15) & $122(5)$ & 63(3) & 63(3) & $9(2)$ & $-1(4)$ & $12(4)$ \\
\hline C(16) & 169(9) & 83(4) & $67(3)$ & $-5(3)$ & $-10(5)$ & $4(5)$ \\
\hline $\mathrm{C}(17)$ & $170(9)$ & 78(4) & $88(4)$ & $0(3)$ & $8(6)$ & $2(6)$ \\
\hline C(18) & 162(10) & 159(9) & $104(6)$ & $11(6)$ & $10(7)$ & $-14(9)$ \\
\hline C(19) & 195(11) & $123(6)$ & $149(7)$ & $54(6)$ & $3(9)$ & 13(8) \\
\hline C(20) & 173(9) & 79(4) & 82(4) & $8(3)$ & $14(5)$ & 12(6) \\
\hline $\mathrm{C}(21)$ & $141(7)$ & $76(3)$ & 78(4) & $3(3)$ & $-2(4)$ & $-6(4)$ \\
\hline C(22) & 131(6) & $67(3)$ & 70(3) & 1(3) & $3(4)$ & $-7(4)$ \\
\hline C(23) & 135(7) & $85(4)$ & $95(4)$ & 2(3) & $-10(5)$ & 1(5) \\
\hline $\mathrm{O}(1)$ & $160(5)$ & 101(3) & $74(2)$ & $-6(2)$ & $-5(3)$ & $14(4)$ \\
\hline $\mathrm{O}(2)$ & 171(6) & 89(3) & $95(3)$ & $-25(2)$ & $-2(4)$ & 22(4) \\
\hline $\mathrm{O}(3)$ & 175(7) & $156(5)$ & $102(4)$ & $-45(4)$ & $-16(5)$ & $52(5)$ \\
\hline $\mathrm{O}(4)$ & $150(5)$ & 109(3) & $96(3)$ & $-21(3)$ & $12(4)$ & $3(4)$ \\
\hline $\mathrm{O}(5)$ & 211(7) & 103(3) & $96(3)$ & $-19(3)$ & 33(5) & $-24(5)$ \\
\hline $\mathrm{O}(6)$ & 197(7) & 74(3) & $122(4)$ & 1(3) & $43(5)$ & $8(4)$ \\
\hline $\mathrm{O}(7)$ & 215(7) & $100(3)$ & 91(3) & $8(3)$ & $25(4)$ & $-14(5)$ \\
\hline$S(1)$ & 132(2) & $78(1)$ & 101(1) & $10(1)$ & $-12(1)$ & $-2(1)$ \\
\hline
\end{tabular}

Table 5. Hydrogen coordinates ( $\left.\mathrm{x} 10^{4}\right)$ and isotropic displacement parameters $\left(\AA^{2} \times 10^{3}\right)$ for sp5036.

\begin{tabular}{lrrrc}
\hline & $x$ & $y$ & $z$ & $U(e q)$ \\
\hline & & & & \\
$H(3)$ & -86 & -1315 & 648 & 120 \\
$H(4)$ & 1581 & -3511 & 1279 & 122 \\
H(5A) & 3644 & -3626 & 407 & 167 \\
H(5B) & 4277 & -4442 & 777 & 167
\end{tabular}




\begin{tabular}{|c|c|c|c|c|}
\hline $\mathrm{H}(6 \mathrm{~A})$ & 7057 & -3240 & 290 & 262 \\
\hline $\mathrm{H}(6 \mathrm{~B})$ & 6998 & -4317 & 236 & 262 \\
\hline $\mathrm{H}(6 \mathrm{C})$ & 7828 & -3864 & 747 & 262 \\
\hline $\mathrm{H}(7 \mathrm{~A})$ & 3569 & -1939 & 1701 & 126 \\
\hline $\mathrm{H}(7 \mathrm{~B})$ & 2926 & -2836 & 2003 & 126 \\
\hline $\mathrm{H}(8)$ & -703 & -2524 & 1887 & 108 \\
\hline $\mathrm{H}(9)$ & 1365 & -1700 & 2580 & 122 \\
\hline $\mathrm{H}(10 \mathrm{~A})$ & -3098 & -1342 & 2353 & 185 \\
\hline $\mathrm{H}(10 \mathrm{~B})$ & -2084 & -1107 & 2890 & 185 \\
\hline $\mathrm{H}(10 \mathrm{C})$ & -2259 & -2137 & 2710 & 185 \\
\hline $\mathrm{H}(11)$ & 2321 & -429 & 2037 & 113 \\
\hline $\mathrm{H}(13 \mathrm{~A})$ & 4394 & 481 & 2734 & 231 \\
\hline $\mathrm{H}(13 \mathrm{~B})$ & 4017 & -508 & 2962 & 231 \\
\hline $\mathrm{H}(13 \mathrm{C})$ & 3420 & 367 & 3287 & 231 \\
\hline $\mathrm{H}(14)$ & -1995 & 208 & 2091 & 109 \\
\hline $\mathrm{H}(18 \mathrm{~A})$ & 2298 & 3726 & 1103 & 212 \\
\hline $\mathrm{H}(18 \mathrm{~B})$ & 3049 & 3041 & 672 & 212 \\
\hline $\mathrm{H}(18 \mathrm{C})$ & 2241 & 2659 & 1203 & 212 \\
\hline $\mathrm{H}(19 \mathrm{~A})$ & -2201 & 3829 & 278 & 234 \\
\hline $\mathrm{H}(19 \mathrm{~B})$ & 220 & 3901 & 105 & 234 \\
\hline $\mathrm{H}(19 \mathrm{C})$ & -599 & 4469 & 580 & 234 \\
\hline $\mathrm{H}(21 \mathrm{~A})$ & -4431 & 816 & 1538 & 118 \\
\hline $\mathrm{H}(21 \mathrm{~B})$ & -4746 & 925 & 943 & 118 \\
\hline $\mathrm{H}(23 \mathrm{~A})$ & -3572 & -571 & 384 & 158 \\
\hline $\mathrm{H}(23 \mathrm{~B})$ & -5717 & -568 & 705 & 158 \\
\hline $\mathrm{H}(23 \mathrm{C})$ & -4085 & -1391 & 758 & 158 \\
\hline
\end{tabular}

Table 6. Torsion angles $\left[^{\circ}\right]$ for sp5036.

\begin{tabular}{lc}
\hline $\mathrm{C}(22)-\mathrm{C}(1)-\mathrm{C}(2)-\mathrm{C}(3)$ & $40.6(13)$ \\
$\mathrm{C}(14)-\mathrm{C}(1)-\mathrm{C}(2)-\mathrm{C}(3)$ & $-128.1(8)$ \\
$\mathrm{C}(22)-\mathrm{C}(1)-\mathrm{C}(2)-\mathrm{C}(8)$ & $-149.1(8)$ \\
$\mathrm{C}(14)-\mathrm{C}(1)-\mathrm{C}(2)-\mathrm{C}(8)$ & $42.2(9)$ \\
$\mathrm{C}(1)-\mathrm{C}(2)-\mathrm{C}(3)-\mathrm{O}(1)$ & $173.5(7)$ \\
$\mathrm{C}(8)-\mathrm{C}(2)-\mathrm{C}(3)-\mathrm{O}(1)$ & $3.2(13)$ \\
$\mathrm{O}(2)-\mathrm{C}(4)-\mathrm{C}(7)-\mathrm{C}(8)$ & $171.4(6)$ \\
$\mathrm{O}(1)-\mathrm{C}(4)-\mathrm{C}(7)-\mathrm{C}(8)$ & $52.8(9)$ \\
$\mathrm{C}(4)-\mathrm{C}(7)-\mathrm{C}(8)-\mathrm{C}(2)$ & $-53.1(7)$ \\
$\mathrm{C}(4)-\mathrm{C}(7)-\mathrm{C}(8)-\mathrm{C}(9)$ & $-178.6(5)$
\end{tabular}




\begin{tabular}{|c|c|}
\hline $\mathrm{C}(3)-\mathrm{C}(2)-\mathrm{C}(8)-\mathrm{C}(7)$ & $26.7(10)$ \\
\hline $\mathrm{C}(1)-\mathrm{C}(2)-\mathrm{C}(8)-\mathrm{C}(7)$ & $-143.7(7)$ \\
\hline $\mathrm{C}(3)-\mathrm{C}(2)-\mathrm{C}(8)-\mathrm{C}(9)$ & $152.5(8)$ \\
\hline $\mathrm{C}(1)-\mathrm{C}(2)-\mathrm{C}(8)-\mathrm{C}(9)$ & $-17.9(11)$ \\
\hline $\mathrm{C}(7)-\mathrm{C}(8)-\mathrm{C}(9)-\mathrm{C}(11)$ & $88.3(8)$ \\
\hline $\mathrm{C}(2)-\mathrm{C}(8)-\mathrm{C}(9)-\mathrm{C}(11)$ & $-34.5(11)$ \\
\hline C(7)-C(8)-C(9)-C(10) & $-148.3(6)$ \\
\hline C(2)-C(8)-C(9)-C(10) & 88.9(8) \\
\hline $\mathrm{C}(8)-\mathrm{C}(9)-\mathrm{C}(11)-\mathrm{C}(12)$ & $-172.8(9)$ \\
\hline C(10)-C(9)-C(11)-C(12) & $65.6(10)$ \\
\hline C(8)-C(9)-C(11)-C(14) & 65.3(9) \\
\hline C(10)-C(9)-C(11)-C(14) & $-56.3(7)$ \\
\hline $\mathrm{C}(14)-\mathrm{C}(11)-\mathrm{C}(12)-\mathrm{O}(3)$ & $31.9(12)$ \\
\hline $\mathrm{C}(9)-\mathrm{C}(11)-\mathrm{C}(12)-\mathrm{O}(3)$ & $-89.9(10)$ \\
\hline $\mathrm{C}(14)-\mathrm{C}(11)-\mathrm{C}(12)-\mathrm{C}(13)$ & $-146.7(8)$ \\
\hline C(9)-C(11)-C(12)-C(13) & $91.5(10)$ \\
\hline C(22)-C(1)-C(14)-C(11) & $-179.8(7)$ \\
\hline $\mathrm{C}(2)-\mathrm{C}(1)-\mathrm{C}(14)-\mathrm{C}(11)$ & $-9.9(9)$ \\
\hline $\mathrm{C}(22)-\mathrm{C}(1)-\mathrm{C}(14)-\mathrm{S}(1)$ & $-59.6(9)$ \\
\hline $\mathrm{C}(2)-\mathrm{C}(1)-\mathrm{C}(14)-\mathrm{S}(1)$ & $110.3(6)$ \\
\hline $\mathrm{C}(12)-\mathrm{C}(11)-\mathrm{C}(14)-\mathrm{C}(1)$ & $-163.1(7)$ \\
\hline $\mathrm{C}(9)-\mathrm{C}(11)-\mathrm{C}(14)-\mathrm{C}(1)$ & $-42.1(9)$ \\
\hline C(12)-C(11)-C(14)-S(1) & $75.4(8)$ \\
\hline C(9)-C(11)-C(14)-S(1) & $-163.6(5)$ \\
\hline $\mathrm{C}(20)-\mathrm{C}(15)-\mathrm{C}(16)-\mathrm{O}(4)$ & 159.7(8) \\
\hline $\mathrm{C}(21)-\mathrm{C}(15)-\mathrm{C}(16)-\mathrm{O}(4)$ & $38.0(10)$ \\
\hline S(1)-C(15)-C(16)-O(4) & $-83.3(10)$ \\
\hline $\mathrm{C}(20)-\mathrm{C}(15)-\mathrm{C}(16)-\mathrm{O}(6)$ & $-21.9(11)$ \\
\hline $\mathrm{C}(21)-\mathrm{C}(15)-\mathrm{C}(16)-\mathrm{O}(6)$ & $-143.6(7)$ \\
\hline $\mathrm{S}(1)-\mathrm{C}(15)-\mathrm{C}(16)-\mathrm{O}(6)$ & $95.0(7)$ \\
\hline $\mathrm{C}(16)-\mathrm{C}(15)-\mathrm{C}(20)-\mathrm{O}(5)$ & $-157.7(11)$ \\
\hline $\mathrm{C}(21)-\mathrm{C}(15)-\mathrm{C}(20)-\mathrm{O}(5)$ & $-36.1(13)$ \\
\hline S(1)-C(15)-C(20)-O(5) & $84.7(12)$ \\
\hline $\mathrm{C}(16)-\mathrm{C}(15)-\mathrm{C}(20)-\mathrm{O}(7)$ & 11.6(13) \\
\hline $\mathrm{C}(21)-\mathrm{C}(15)-\mathrm{C}(20)-\mathrm{O}(7)$ & 133.2(9) \\
\hline S(1)-C(15)-C(20)-O(7) & $-105.9(9)$ \\
\hline $\mathrm{C}(16)-\mathrm{C}(15)-\mathrm{C}(21)-\mathrm{C}(22)$ & $-167.5(5)$ \\
\hline $\mathrm{C}(20)-\mathrm{C}(15)-\mathrm{C}(21)-\mathrm{C}(22)$ & $67.9(7)$ \\
\hline $\mathrm{S}(1)-\mathrm{C}(15)-\mathrm{C}(21)-\mathrm{C}(22)$ & $-49.2(7)$ \\
\hline C(2)-C(1)-C(22)-C(23) & 15.2(13) \\
\hline
\end{tabular}




$\begin{array}{lc}\mathrm{C}(14)-\mathrm{C}(1)-\mathrm{C}(22)-\mathrm{C}(23) & -176.2(7) \\ \mathrm{C}(2)-\mathrm{C}(1)-\mathrm{C}(22)-\mathrm{C}(21) & -164.9(7) \\ \mathrm{C}(14)-\mathrm{C}(1)-\mathrm{C}(22)-\mathrm{C}(21) & 3.7(11) \\ \mathrm{C}(15)-\mathrm{C}(21)-\mathrm{C}(22)-\mathrm{C}(1) & 55.6(9) \\ \mathrm{C}(15)-\mathrm{C}(21)-\mathrm{C}(22)-\mathrm{C}(23) & -124.5(7) \\ \mathrm{C}(2)-\mathrm{C}(3)-\mathrm{O}(1)-\mathrm{C}(4) & -6.2(13) \\ \mathrm{O}(2)-\mathrm{C}(4)-\mathrm{O}(1)-\mathrm{C}(3) & -143.3(6) \\ \mathrm{C}(7)-\mathrm{C}(4)-\mathrm{O}(1)-\mathrm{C}(3) & -22.5(11) \\ \mathrm{O}(1)-\mathrm{C}(4)-\mathrm{O}(2)-\mathrm{C}(5) & -65.2(8) \\ \mathrm{C}(7)-\mathrm{C}(4)-\mathrm{O}(2)-\mathrm{C}(5) & 172.2(6) \\ \mathrm{C}(6)-\mathrm{C}(5)-\mathrm{O}(2)-\mathrm{C}(4) & 162.3(9) \\ \mathrm{O}(4)-\mathrm{C}(16)-\mathrm{O}(6)-\mathrm{C}(17) & 178.0(9) \\ \mathrm{C}(15)-\mathrm{C}(16)-\mathrm{O}(6)-\mathrm{C}(17) & -0.5(12) \\ \mathrm{O}(7)-\mathrm{C}(17)-\mathrm{O}(6)-\mathrm{C}(16) & 31.5(12) \\ \mathrm{C}(18)-\mathrm{C}(17)-\mathrm{O}(6)-\mathrm{C}(16) & -92.5(10) \\ \mathrm{C}(19)-\mathrm{C}(17)-\mathrm{O}(6)-\mathrm{C}(16) & 143.7(9) \\ \mathrm{O}(5)-\mathrm{C}(20)-\mathrm{O}(7)-\mathrm{C}(17) & -168.3(11) \\ \mathrm{C}(15)-\mathrm{C}(20)-\mathrm{O}(7)-\mathrm{C}(17) & 21.6(15) \\ \mathrm{O}(6)-\mathrm{C}(17)-\mathrm{O}(7)-\mathrm{C}(20) & -43.2(13) \\ \mathrm{C}(18)-\mathrm{C}(17)-\mathrm{O}(7)-\mathrm{C}(20) & 80.7(12) \\ \mathrm{C}(19)-\mathrm{C}(17)-\mathrm{O}(7)-\mathrm{C}(20) & -156.3(11) \\ \mathrm{C}(16)-\mathrm{C}(15)-\mathrm{S}(1)-\mathrm{C}(14) & 116.9(5) \\ \mathrm{C}(20)-\mathrm{C}(15)-\mathrm{S}(1)-\mathrm{C}(14) & -121.1(5) \\ \mathrm{C}(21)-\mathrm{C}(15)-\mathrm{S}(1)-\mathrm{C}(14) & -2.3(5) \\ \mathrm{C}(1)-\mathrm{C}(14)-\mathrm{S}(1)-\mathrm{C}(15) & 52.8(6) \\ \mathrm{C}(11)-\mathrm{C}(14)-\mathrm{S}(1)-\mathrm{C}(15) & 177.0(4) \\ & \\ & \\ & \\ & \\ & \end{array}$

Symmetry transformations used to generate equivalent atoms: 